UNIVERSITY OF OXFORD

\title{
Poetik und Politik der Lesbarkeit in der deutschen Literatur
}

\author{
Benjamin Schaper \\ Oriel College \\ Hilary Term 2016
}

A thesis submitted for the degree of D.Phil. in Medieval and Modern Languages 
Meiner Schwester gewidmet.

Damit sie endlich Ruhe gibt.

Eigentlich aber, weil ich sie so sehr lieb habe.

का 


\title{
Poetik und Politik der Lesbarkeit in der deutschen Literatur
}

\author{
Benjamin Schaper \\ Oriel College \\ Hilary Term 2016 \\ D.Phil. in Medieval and Modern Languages
}

\begin{abstract}
Short Abstract
In 1990, German literary critics agreed that the end of the Cold War should mark the end of politically committed post-war literature. The political caesura prompted a debate about the future of German literature during which the concept of 'readability' evolved as a contested issue. It was championed in particular by the author Matthias Politycki and the publishers Uwe Wittstock and Martin Hielscher. Ever since, 'readability' has remained a benchmark for authors and critics alike in the battle for value and success.

The thesis will establish a theoretical basis for 'readability' that draws on narratology, the Aristotelian concept of 'mimesis', classical rhetoric, and the poetics of contemporary authors who explicitly engage with 'readability'. Discussion will centre on the novel since this genre has been the focus of debate ever since the novel gained prominence with the rise of the reading middle classes in the eighteenth century. An analysis of the historical role of 'readability' will demonstrate that the debate as it manifested itself around 1990 developed out of a specifically German tradition, in which authors and critics alike viewed it as potentially in conflict with true art.

The thesis will demonstrate that 'readability' is key to understanding the debates about German literature in an era of globalisation when readers are more attracted to works by foreign authors than to works by German ones. It will examine how writers such as Helmut Krausser, Daniel Kehlmann, and Thomas Glavinic have exploited the opportunities of the changed parameters by writing and promoting 'readable' books. It will further explore to what extent 'readability' has opened up new avenues even for authors like Felicitas Hoppe and Ulrike Draesner, who distrust the quest for 'readability'. The thesis will conclude with a reflection on the prospects for 'readability' in the current literary landscape in Germany.
\end{abstract}




\title{
Poetik und Politik der Lesbarkeit in der deutschen Literatur
}

\author{
Benjamin Schaper \\ Oriel College \\ Hilary Term 2016 \\ D.Phil. in Medieval and Modern Languages
}

\section{Long Abstract}

The thesis establishes 'readability' as a key poetological category that gained prominence in the German literary landscape at around 1990 in the context of the 'Literaturstreit', a wide-ranging debate about the way forward for German literature after the post-war era. The introduction addresses the hypothesis of the author Matthias Politycki that the championing of 'readability' has brought about a change of paradigm in German literature. The concept of a paradigm shift will serve as a starting point for the subsequent analysis, drawing on Thomas S. Kuhn's paradigm-theory as developed in The Structure of Scientific Revolutions (1962). This will provide a framework for investigating the value of 'readability' as a stimulus for debate about the relationship between the author, text and reader. The Introduction will discuss current research on 'readability' and identify a lack of critical engagement with this concept as a poetologically significant phenomenon. It will elucidate the methodological approach that underpins the thesis and thereby lay the groundwork for the subsequent chapters.

The first main chapter will establish the theoretical parameters of the concept of 'readability'. The analysis of 'readability' as debated in the German context since the 'Literaturstreit' will initially be situated in contemporary narratology, focusing on aspects that highlight effective engagement of the reader. These in turn draw on key categories discussed in classical poetics and rhetoric, which will throughout the thesis serve as a benchmark for the concept of 'readability'. The poetological work of American author Jonathan Franzen - who serves as a role-model for authors Helmut Krausser, Daniel Kehlmann and Thomas Glavinic - establishes the contemporary importance of 'readability' in international terms. Franzen's essay 'Mr. Difficult' (2002) will provide an example of the continuing relevance of the classical precepts discussed for contemporary poetics. Particular interest will be given to Franzen's 'contract model', which identifies the 'readable' text as the necessary basis for the fruitful relationship between author and reader. 
The purpose of establishing these parameters at the outset is to create a broadly based frame of reference for the subsequent chapters, allowing continuities to emerge as well as shifts of emphasis. Moreover, they serve to highlight the importance of 'readability' as a concept that has retained significance across time and in different cultural, political and economical contexts, continuing to serve as a productive benchmark for contemporary writers, readers, critics and other participants in the literary process.

For the concern in this project is not to establish congruences or differences between authorial intention and reception by readers, but to identify the criteria that are in play in debates about 'readability' between all participants in the literary process. Thereby it becomes clear that 'readability' is not a static category but one that is intrinsically dynamic. Analysis of the concept and its application will show that is subject to permanent change and reshaping, so that close analysis of individual works becomes crucial for understanding its force.

Chapter 2 examines the concept of 'readability' historically in order to demonstrate that the issues debated since 1990 in terms of a 'new' readability have in fact engaged critics and writers repeatedly in the course of German literary history. It will show that the notion of competition was not only intrinsic to the 'Literaturstreit' but that 'readability' has been vehemently contested - notably within the binary division between highbrow 'Kunst' and lowbrow 'Trivialliteratur' - since it became a relevant issue with the rise of the novel in the eighteenth century. Other key moments discussed are Georg Wilhelm Friedrich Hegel's notion of the 'freies poetisches Kunstwerk' as developed in his Vorlesungen über die Ästhetik in the first half of the 19th century; the debate about narrative literature in the second half of the same century (e.g. 'Gartenlaubenstil'); the 'difficult' avantgarde literature in the period of Modernism; Theodor Adorno's and Max Horkheimer's attack on the 'Kulturindustrie', which excludes any piece of fiction aiming at a broad audience from being art; and the didactic aims guiding writers in both East and West Germany during the postwar era. This establishes the historical context for the virulent debate around 1990.

Chapter 3 argues that while the discussion about 'readability' was already a wellestablished feature of the German literary tradition, it gained a new and important role as a point of reference at the time of unification. The chapter identifies the different voices in the debate of 1990 and the respective vested interests in order to identify the 
precepts concerning 'readability' articulated at that cultural and political turning point. It will initially focus on the rejection of a so-called 'Gesinnungsästhetik' in German postwar literature, which gave literature the task to deal with the moral burden of Nazism. The term was coined by philosopher and critic Karl Heinz-Bohrer, and it was then later used by fellow critics Ulrich Greiner and Frank Schirrmacher in order to attack Christa Wolf's semi-autobiographical novella Was bleibt (1990). The chapter will then briefly examine the changing market developments of that time, which inspired critics and authors alike to search for a new aesthetic to guide German literature into the future. The chapter closely examines the precepts and arguments used by critics in the course of the debate, relating these to the poetological categories outlined in the theoretical chapter and the historical developments traced in Chapter 2. The debate about 'readability' in 1990 serves as a benchmark for investigating how the landscape has changed since then, and how authors have responded to that change. It argues that German authors have turned to 'readability' in order to achieve national and especially international success. It will be shown that such success has become an explicit goal for German authors since 1990, and that authors are now pressing critics and the media to promote their 'readable' works to an international readership.

Chapter 4 will examine the interplay between theory and practice. In doing so, it will consider the poetological as well as the fictional works of Helmut Krausser, Daniel Kehlmann and Thomas Glavinic. It initially focuses on Helmut Krausser, who has fought for a 'readable' literature since the early 1990s, considering himself and his writing as a project designed to explore various types of 'readable' text. The chapter examines Krausser's reflections on the competing poetological categories of the 'Literaturstreit' and investigates how reviewers discussed Krausser's novels as a reaction to the promotion of 'readability'. The chapter investigates what Krausser means by 'readability', and it will situate his project in the tradition discussed in Chapter 2. The chapter argues that while a philosophically oriented aesthetics distrusts public promotion of effect as being in conflict with 'free art' there is no such tension in Krausser's works. It will be argued that they may be seen as part of a 'rhetorical turn' in the German literary landscape since the 1990s, which has opened up new opportunities for German writers.

The chapter then goes on to examine the collaboration between Helmut Krausser and Kehlmann, who have promoted each other's works in the media as well as in 
academic journals. The chapter argues that in taking on academia as well as the ordinary reading public, the two authors have set out to effect a comprehensive reconfiguration of literary values, and engaged head-on with the hierarchies established by Idealism in the early 19th century and confirmed by its proponents around 1990. The extensive debate about Kehlmann's status as a global bestselling author permits analysis of the features of his success and serves to show how espousing 'readability' has allowed him to appeal to readers both in Germany and on the global stage. The chapter argues that beyond promoting his own work successfully, the cooperation with Krausser has been successful in opening up avenues that other authors like Glavinic have benefited from as well. After succeeding in establishing himself in the literary landscape, Kehlmann has in turn promoted Glavinic. The chapter will demonstrate how the cooperation between the authors has enhanced their impact on the literary landscape, and given 'readability' a higher profile.

The chapter concludes with a close reading of the poetological works of the three authors and the practical implementation of 'readability' in Krausser's novel Nicht ganz schlechte Menschen (2012), Kehlmann's Die Vermessung der Welt (2005) and Glavinic's Das größere Wunder (2013). Kehlmann's global bestseller functions as a central point of reference since it can be seen as symptomatic for the change triggered by 'Neue Deutsche Lesbarkeit' as highlighted by Uwe Wittstock's eulogy at the 'WeltLiteraturpreis' (2006). In Nicht ganz schlechte Menschen, Krausser takes the debate about 'readability' into his primary fictional work. Meta-fictionally, Krausser juxtaposes different approaches to literature and ends his novel with the victory of a literary programme based on 'readability'. Meanwhile Glavinic's Das größere Wunder operates within the genre of the love-story. The analysis shows that the proponents of 'readability' have had a profound effect on the German literary landscape regarding the boundaries between 'high' and 'low' literature. Elements that have traditionally been attributed to 'low' have now become acceptable within the realms of 'high literature'.

Chapter 5 will selectively address critical positions towards 'readability', focussing on Felicitas Hoppe and Ulrike Draesner as authors whose literary projects differ markedly both from each other and from the projects of the writers discussed in the preceding chapter. They serve as examples for a productive engagement with the literary parameters that inform the programme of 'readability' used by Krausser, Kehlmann and Glavinic. The chapter initially considers how their projects relate to the precepts 
encapsulated in the concept of 'readability', and analyses their theoretical critique of easy 'readability'. It will then go on to investigate how Hoppe and Draesner in fact find creative and productive ways to engage with the aim of 'readability' in their fiction. Hoppe explores the genre of children's fiction in order to engage with 'readability' in Iwein Löwenritter (2008). The chapter will identify her strategies and demonstrate that she also applies these to her adult fiction, which will be represented by her novel Hoppe (2012). Draesner's theoretical and fictional work is shown to be powerfully informed by the Aristotelean tradition of realism. In her novel Sieben Sprünge vom Rand der Welt (2014) and the bigger project of which it forms a part, she uses the internet to reconfigure the relationship between author, reader and text while ensuring the 'readability' of her novel. The examination of the ways in which both authors approach 'readability' will confirm the profound impact of the concept on German contemporary writers and highlight the dynamics of its contribution to literary practice.

The thesis will conclude with reflections on the extent to which 'readability' has retained its significance in the German-speaking literary landscape since the 'Literaturstreit'. The conclusion will provide a short synopsis of research results that will open up a final discussion of the prospects of 'readability'. It will be argued that the rise of 'readability' as a poetological premise in the German context has not only led to a profound shift in aesthetic precepts but helped to establish new strategies for the promotion of literature in a context marked by 'eventisation'. Examples are provided by the programmes of events in the 'Literaturhäuser' and the newly created German Book Award (since 2005), which emulates the National Book Award and the Booker Prize in order to keep authors and their works in the public eye. The conclusion proposes further research questions that cannot be addressed within the scope of this thesis but which could yield a deeper understanding of the notion of 'readability'. The thesis will conclude with a brief look at the young German author Benedict Wells as a representative of the new generation after Krausser, Kehlmann, Glavinic, Hoppe and Draesner. Wells's essay 'Und, wie geht die Geschichte weiter? Sag schon!' (2012) shows that for him, 'readability' is an artistically valuable category, which he no longer regards as contested - he takes it for granted. 


\section{Danksagungen}

Vor allem danke ich meiner Doktormutter Katrin Kohl. Ich danke Dir dafür, dass Du mir dazu den Anstoß gegeben hast, das bei unserem ersten Treffen leichtfertig dahingesagte Wort 'Lesbarkeit' in seiner zunächst unscheinbaren Komplexität ernst zu nehmen. Ich bedanke mich für die Begeisterung und die Energie, die Du bis zuletzt in dieses Projekt investiert hast. Zudem danke ich Dir für die wunderbaren Erfahrungen, die ich mit dem German Network machen durfte, und für Deine Unterstützung des German Play, das mir so sehr am Herzen liegt.

Many thanks to Oriel College for funding my research and conference trips (in diesem Kontext auch vielen Dank an meine Cousinen Annette und Julia für die nette Beherbergung), for the hospitality and the friends I was able to make during the last years. I would like to thank the supportive porters and employees of the Academic Office as well as Sash and Cheryl.

I would like to give special thanks to my college advisor Annette Volfing for not only caring about my work but also about my well-being. Thank you very much for giving me the opportunity to teach these two courses that have given me so much pleasure and thank you for making sure that I did not make outrageous claims about Hartmann.

Meinem Freund und ehemaligen Dozenten Erik Schilling verdanke ich die Passion für die Gegenwartsliteratur und meine ersten Kontakte mit Krausser, Kehlmann und Draesner. Diese Arbeit ist nicht zuletzt eine Konsequenz Deiner begeisternden Seminare.

Im Gedenken an Rudi Gürtner, dessen bedingungslose Unterstützung und Güte aus den unermesslich bedeutsamen Räumen zwischen den Zeilen, Wörtern und Buchstaben dieser Arbeit sprechen.

Mein spezieller Dank gilt den Teams der Taylor Institution Library, des Deutschen Literaturarchivs in Marbach und der Frankfurter Poetikvorlesungen, insbesondere Esther Delp.

Darüber hinaus bin ich meinen eifrigen Lesern zu Dank verpflichtet: Katharina Laszlo (auch für die vielen netten Schoki-Treffen), Jonas Harnau (auch für das traditionelle Haxn-Schmankerl), Michael Pfister (auch für die alljährliche Pilgerfahrt in die Mondstraße nach Fürstenfeldbruck), Thomas von Pluto-Prondzinski (auch dafür, 
dass Du mein Mittstreiter im Kampf für Lesbarkeit und Unterhaltung bist), Jonathan Schindler (auch für den Kaiser), Andi Kratzert (auch für die zahlreichen anregenden Unterhaltungen), Stephan Ehrig (auch für das schöne Wochenende in Bristol - ich freue mich schon auf den nächsten XD-Double-Act) und Alex Abel (auch für so vieles andere vom vorweihnachtlichen Kinogang bis zum Zitronengesicht). Außerdem danke ich Michael Navratil für den Tipp zu Tieck und seine generelle Begeisterung für das Projekt.

Alexander Pfefferler, meinem alten Freund, dessen 'Namensgenosse' in dieser Arbeit eine solch große Rolle spielt, danke ich für die anregenden Stunden am Tresen des Simpl.

Melissa Schuh: Vielen Dank für Dein offenes Ohr zu jeder Tages- und Nachtzeit, dein Feedback zu meinen Nebenprojekten und die beiden fantastischen Abende an der Queen Mary.

Ich bedanke mich bei 'Meister' Jacobi für seine Freundschaft, die unterhaltsamen Einführungen in die Vorzüge der Stadt Düsseldorf und besonders für seine Begeisterung für das German Play, ohne die das Projekt heute nicht wäre, was es ist.

To João Jorge and Colleen Curran, my two oldest friends in this magical town of dreaming spires: What a ride this has been since we met in St. Hilda's all these years ago. You guys have been my rocks. Thanks a lot for everything and good luck with the last push of your theses!

Merci à Claire Deligny d'être mon amie, ma co-souffrante et ma complice. Je n'oublierai jamais nos heures passées ensemble dans la salle d'étude (avec Cecil $\theta$ ), sur Internet et dans ton bureau - sans compter l'UPP et nos nombreux pique-niques. Elles ont été non seulement parmi les heures les difficiles de la ma vie, mais encore et surtout, quelques-unes des heures les plus heureuses. Sans toi, je me serai senti bien seul lors de ces derniers mois.

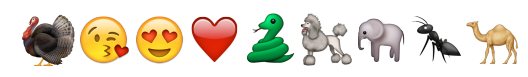

Meiner kleinen, aber für mich umso wichtigeren Familie verdanke ich die Freiheit, das zu tun, was ich liebe. Ich bedanke mich bei meinem Vater, meinem Cousin, dem Griechen, meinem Onkel, meinen Tanten, meinen Omas, meiner Schwester, Dir Op und meiner Mutter dafür, dass ihr mich in den letzten Jahren vor allen äußeren Widrigkeiten bewahrt habt, sodass ich mich einzig auf meine Arbeit konzentrieren konnte. 


\section{Inhaltsverzeichnis}

\section{Einführung}

Seite 2-18

\section{Kapitel 1}

Lesbarkeit als theoretisches Prinzip und der Vertrag zwischen Autor und Leser Seite 19-38

\section{Kapitel 2}

Lesbarkeit in der Tradition deutscher Erzählliteratur

Seite $39-87$

\section{Kapitel 3}

Der Literaturstreit und das Programm der 'Neuen Deutschen Lesbarkeit'

Seite 88-112

\section{Kapitel 4}

Helmut Krausser, Daniel Kehlmann, Thomas Glavinic und der Kampf für die 'gute Sache'

Seite 113-181

\section{Kapitel 5}

Alte und neue Formen der Lesbarkeit. Felicitas Hoppe und Ulrike Draesner

Seite 182-231

\section{Perspektiven}

Seite $232-241$

\section{Bibliographie}

Seite 242-260 


\section{Einführung}

Die politische Zäsur des Mauerfalls und der anschließenden Wiedervereinigung Deutschlands führte im Jahre 1990 zu einem Literaturstreit, der die zukünftige Ausrichtung der deutschen Literatur nachhaltig prägen sollte. Einflussreiche Vertreter diverser Institutionen des Kulturbetriebs beteiligten sich an einer virulenten Debatte, die hauptsächlich in den deutschen Feuilletons ausgetragen wurde und $\mathrm{zu}$ einer tiefgreifenden Veränderung der Literaturlandschaft beitrug. Die Streitenden plädierten zunächst für die Zurückweisung einer vor allem politisch und moralisch ambitionierten Nachkriegsliteratur. ${ }^{1}$ Diese machten sie dafür verantwortlich, dass die deutsche Literatur an ästhetischer Qualität eingebüßt habe. In der Folge avancierte 'Lesbarkeit' zu einem zentralen poetologischen Begriff, welcher der deutschen Literatur sowohl Qualität als auch Erfolg bei Lesern im In- und Ausland sichern sollte. Den Impuls zur Hervorhebung der Lesbarkeit und $\mathrm{zu}$ deren Etablierung als zentrale poetologische Kategorie lieferten der Kritiker und Verleger Uwe Wittstock sowie der Autor Matthias Politycki als Hauptvertreter einer 'Neuen Deutschen Lesbarkeit'. ${ }^{2}$ Diese sollte dem Wechselspiel zwischen Autor, Text und Leser durch einen gezielt gesuchten Publikumsbezug und die strategische Verquickung von Praxis und Theorie produktive Energie verleihen. Dabei plädierten sie für einen Anschluss an die anglo-amerikanische Postmoderne und den Rückgriff auf bewährte Mittel der Erzähltradition. ${ }^{3}$ Die Lesbarkeitsdebatte in Deutschland entstand demnach einerseits aus einer spezifisch deutschen historischen Entwicklung heraus und andererseits aus einer Auseinandersetzung mit grundsätzlichen Fragen der literarischen Theorie und Praxis, die sich am internationalen Kontext orientierte.

Die für den Lesbarkeitsbegriff relevanten Bezugssysteme sowie deren Vertreter sollen im Anschluss eingeführt und situiert werden. Zunächst soll der Begriff 'Lesbarkeit' definiert und $\mathrm{zu}$ der in den 1990er Jahren prominent gewordenen Bezeichnung einer 'Neuen Deutschen Lesbarkeit' bzw. 'Neuen Lesbarkeit' in Bezug

\footnotetext{
1 Vgl. v.a. Greiner 1991, Schirrmacher 1990 und Schirrmacher 1991.

2 Vgl. u.a. Taberner 2002, Taberner 2005 und Taberner 2007.

3 Dies wird insbesondere aus Wittstock 1995 ersichtlich. Eine detaillierte Analyse von Wittstocks Band sowie des literarischen Programms der 'Neuen Deutschen Lesbarkeit' wird in Kapitel 3 der vorliegenden Arbeit erfolgen.
} 
gesetzt werden. Polityckis Ausführungen dienen dann als Anhaltspunkt für das Rekurrieren auf die Tradition, Wittstocks für die Orientierung an der internationalen Postmoderne. Zusammen mit einer Untersuchung der bisherigen Forschung zur Lesbarkeit wird so eine Grundlage für die zu leistende Auseinandersetzung mit Lesbarkeit als theoretischem Begriff und praxisbezogenem Wirkungsprinzip geschaffen. Die Struktur der vorliegenden Arbeit soll zum Abschluss der Einführung dargelegt werden.

Lesbarkeit stellt ein poetologisches Prinzip dar, dessen komplexe theoretische Grundzüge im ersten Kapitel etabliert und definiert werden sollen. Auf diese Weise soll eine Unterscheidung sowohl zur gängigen Semantik des Wortes - des 'Lesbarseins'4 als auch zur Debatte in den 1990er Jahren vorgenommen werden. Politycki führte den Begriff der 'Neuen Deutschen Lesbarkeit' in Anlehnung an den germanistischen Teilbereich der 'Neueren Deutschen Literatur' in der Vorrede seiner Anthologie Die Farbe der Vokale (1998) ein, in der er seine essayistischen Arbeiten zu Lesbarkeit in den 1990er Jahren dokumentiert. ${ }^{5}$ Er bezeichnet damit ein auf Lesbarkeit gründendes Literaturprogramm. Später verwendet er den Begriff verkürzt als 'Neue Lesbarkeit', doch erscheint die spezifische Zuordnung zum deutschsprachigen Kontext als sinnvoll, da, wie das historische Kapitel zeigen wird, die Vorbehalte gegenüber Lesbarkeit einer spezifisch deutschen philosophisch-literarischen Tradition entstammen. ${ }^{6}$ Wenn also im Laufe der vorliegenden Arbeit von der 'Neuen Deutschen Lesbarkeit' die Rede sein wird, dann meint dies dezidiert das in den 1990er Jahren im deutschsprachigen Raum erörterte Literaturprogramm, während Lesbarkeit allgemeiner als ein zwar vom jeweiligen zeitgeschichtlichen Kontext inspiriertes, jedoch an sich universelles Prinzip gelten kann.

Die Verortung der 'Neuen Deutschen Lesbarkeit' in den 1990er Jahren erfolgt insbesondere über den Aspekt des 'Neuen'. Armand D'Angour hebt in seiner Studie zum Prinzip der 'novelty' im antiken Griechenland (2011) unterschiedliche Motivationen hervor, aufgrund derer sich Gruppierungen auf das Neue berufen:

\footnotetext{
4 Zur allgemeinen Bedeutung von 'lesbar', siehe Bedeutung 2: 'verständlich geschrieben, sodass die Lektüre keine Schwierigkeiten bereitet' (Duden 2016).

${ }^{5}$ Vgl. Politycki 1998 f, S. 7.

${ }^{6}$ Ebd.
} 
Some may wish to draw attention to genuine innovations; others will seek to impress or persuade their audiences or fellow professionals by such claims. [...] Each passage containing such a claim must be considered on its own terms; a bald compilation of assertions of novelty [...] is apt to conceal a wide variation of the kind of novelty to which appeal is made. 7

Politycki impliziert mit der Betonung des 'Neuen' für das Programm der 'Neuen Deutschen Lesbarkeit' eine historische Dimension und betont zugleich die Ausnahmestellung der gegenwärtigen Erscheinungsform von Lesbarkeit im deutschsprachigen Kontext. Demnach dient der Begriff des Neuen zum einen dazu, das Programm der 'Neuen Deutschen Lesbarkeit' durch die Tradition theoretisch zu legitimieren. Zum anderen dient es der Modifizierung eines traditionellen Lesbarkeitsbegriffs, wobei das Neue eine Besonderheit der angewandten Techniken herausstellt. ${ }^{8}$ Dies verweist auf die dem Lesbarkeitsbegriff inhärente Dynamik, die ihn dazu befähigt, sich verschiedenen historischen Kontexten anzupassen.

Im Kontext der 1990er Jahre rückt Politycki das Programm der 'Neuen Deutschen Lesbarkeit' durch dessen Bezeichnung als 'Wirkungsästhetik' in das Umfeld der Rhetorik. ${ }^{9}$ Für den Lesbarkeitsdiskurs erweist sich Andreas Böhns Definition der 'Wirkungsästhetik' in Kontrast zur 'Rezeptionssästhetik' als produktiv: Die tatsächlichen Rezeptionsbedingungen des jeweiligen Lesers werden ausgeblendet und das 'Wirkungspotenzial als Gesamtheit der die Rezeption aktivierenden, steuernden und restringierenden Textelemente und -strukturen' fokussiert. ${ }^{10}$ Für Lesbarkeit gilt es somit Textstrategien zu identifizieren, die spezifisch darauf ausgerichtet sind, dem Leser die Lektüre zu erleichtern und das Textverständnis zu gewährleisten.

Um die leichte Lesbarkeit eines Textes zu sichern, das Publikum für einen Text zu gewinnen und den Text dabei qualitativ ansprechend zu gestalten, eröffnet Politycki ein grundlegendes, seit der Antike diskutiertes Spannungsverhältnis. Er entwirft die Idealvorstellung eines auf Lesbarkeit gründenden Romans, die dem Prinzip einer

\footnotetext{
7 D’Angour 2011, S. $190 \mathrm{f}$.

8 Politycki macht geltend, dass es Lesbarkeit 'in allen Jahrhunderten aller literarischen Entwicklungen immer wieder einmal gegeben hat', und spricht deswegen im Kontext der 1990er Jahre von einer 'uralte[n] Lesbarkeit, herbeigeführt mit neuen, nachmodernen Mitteln.' (Politycki 1998 f, S. 7).

9 Ebd., S. 9.

10 Böhn 2003, S. 852.
} 
'Simplifizierung bei gleichzeitiger Verkomplizierung' folgen soll. ${ }^{11}$ Dies spricht für einen dynamischen Prozess, in dem vereinfachende und erschwerende Elemente in ständigem Wechselspiel zur Mitte streben. Aristoteles wendet diese Strategie in seiner Poetik hinsichtlich der idealen sprachlichen Gestaltung an. Zwar sind die beiden Theorien aufgrund der unterschiedlichen Anwendungsbereiche nicht unmittelbar vergleichbar - während sich Aristoteles ausschließlich auf die Stilistik bezieht, wendet Politycki das Prinzip auf die Gesamtheit des Romans an -, aber Aristoteles' Ausführungen im 22. Kapitel der Poetik liefern fundierte Erkenntnisse über die Funktionsweise dieses Prozesses:

Die vollkommene sprachliche Form ist klar und zugleich nicht banal. Die sprachliche Form ist am klarsten, wenn sie aus lauter üblichen Wörtern besteht; aber dann ist sie banal. [...] Die sprachliche Form ist erhaben und vermeidet das Gewöhnliche, wenn sie fremdartige Ausdrücke verwendet. [...] Doch wenn jemand nur derartige Wörter verwenden wollte, dann wäre das Ergebnis ein Rätsel oder ein Barbarismus. [...] Man muss also die verschiedenen Arten irgendwie mischen. Denn die eine Gruppe bewirkt das Ungewöhnliche und Nicht-Banale [...]; der übliche Ausdruck hingegen bewirkt Klarheit. ${ }^{12}$

Klarheit steht für ein leichtes Textverständnis, während eine nicht banale Ausdrucksweise für einen Qualitätsanspruch steht. Beide Pole sind Aristoteles zufolge mit Risiken behaftet, da es bei zu großer Klarheit an Qualität und bei zu großer Komplexität an Verständlichkeit fehlt. Hinsichtlich letzterer führt er mit dem Gewöhnlichen bzw. Bekannten einen Breitenwirkung fördernden Aspekt an. 'Erhaben' verweist hier höhenmetaphorisch auf Überlegenheit, jedoch resultiert eine Überstrapazierung dieser Elemente in einem 'Rätsel'. Dies suggeriert Mühe bei der Entschlüsselung eines Textes und im Extremfall die Unmöglichkeit des Verständnisses. Aristoteles warnt somit zugleich vor einer banal einfachen und vor einer rätselhaft unverständlichen Stilistik und plädiert wie in seiner Rhetorik für einen Mittelweg. ${ }^{13}$ Die Kombination aus klarer Verständlichkeit und Qualitätsanspruch soll einen Text sowohl verständlich als auch stilistisch interessant machen. Dieses Spannungsverhältnis zwischen Verständlichkeit und Qualität ist für den Lesbarkeitsbegriff konstitutiv.

Während Polityckis Prinzip die historische Dimension des Lesbarkeitsbegriffs der

\footnotetext{
11 Politycki 1998 d, S. 39.

12 Aristoteles 1994, 22, S.71-73.

13 Vgl. Aristoteles 2007, III, 25, S.183.
} 
1990er Jahre fruchtbar macht, kümmert sich Wittstock in Leselust (1995), seinem programmatischen Plädoyer für Lesbarkeit und Unterhaltung, um dessen internationale Komponente. Insbesondere bezieht er sich auf den Essay des amerikanischen Anglisten und Amerikanisten Leslie A. Fiedler Cross the Border - Close the Gap (1970), der die literarischen Tendenzen der Postmoderne artikuliert. Fiedler hatte seine Theorie zuerst bei einem Symposium an der Albert-Ludwigs-Universität in Freiburg (1968) vorgestellt. Er führte seine für die Literaturlandschaft der Vereinigten Staaten entwickelten Ausführungen in Deutschland ein und suchte, seine deutschen Kollegen $\mathrm{zu}$ deren Übernahme zu gewinnen. Stefan Höppner und Jörg Kreienbrock sprechen deswegen in ihrer Studie zum literarischen und kulturellen Austausch zwischen Deutschland und den USA (2015) von einem 'entscheidende[n] [Impuls] des [...] transatlantischen Kulturtransfers'. ${ }^{14}$ Wittstock befasst sich damit, dass Fiedler bei der Vorstellung seiner Theorie in Freiburg und auch danach in Deutschland wegen der angestrebten Grenzüberschreitung und der Lückenschließung zwischen 'ernster Literatur' und 'Unterhaltungsliteratur' 'die Gemüter [erregt]' habe. ${ }^{15}$ Während die Unterscheidung zwischen E- und U-Literatur beispielsweise in der antiken Legitimierung von Unterhaltung durch den ernsthafteren Zweck der Didaktik angelegt ist, prägt sich die Dichotomie in Deutschland besonders im Zeitraum der Wende zum 19. Jahrhundert im Idealismus aus, wobei die Unterhaltungsliteratur der ernsten Literatur qualitativ untergeordnet wurde - so beispielsweise von den Gebrüdern Schlegel. ${ }^{16}$ Fiedler, der die Distinktion zwischen den beiden Kategorien ursprünglich in der amerikanischen Literatur aufzulösen gedachte, verbindet mit ihnen zudem eine tendenziell gespaltene Leserschaft. Dies exemplifiziert er am Beispiel des französischen Autors Boris Vian: '[He] managed to straddle the border, if not quite close the gap between high culture and low [...]. [Such] a closing of the gap between elite and mass culture is precisely the function of the Novel'. ${ }^{17}$ Fiedler rekurriert auf Höhenmetaphorik, um die Qualität von massenkompatibler Literatur $\mathrm{zu}$ bestimmen. Massenkompatibilität soll durch einen Mittelweg zwischen hoher und niedriger Literatur erreicht werden, um qualitativen

\footnotetext{
14 Höppner/Kreienbrock 2015, S. 6.

15 Wittstock 1995, S. 53.

16 Vgl. dazu Fauser 2003, S. 729 sowie Kohl 2007 b, u.a. S. 457, 575 und 645. Vgl. zu diesem Thema Kapitel 2.

17 Fiedler 1971, S. 468.
} 
Ansprüchen gerecht zu werden und eine breite Masse anzusprechen, wodurch Fiedler ein Spannungsfeld zwischen Erfolg und Qualität eröffnet. Dieses Spannungsfeld ist in der deutschen Nachkriegsliteratur besonders ausgeprägt und wurde speziell im Kontext des Literaturstreits als Herausforderung wahrgenommen. Dies erklärt Wittstocks Beobachtung zu den Reaktionen auf Fiedler in Deutschland. Das Spannungsverhältnis liegt generell in der anhaltenden Bedeutung idealistischer Literaturvorstellungen begründet, vor allem aber kommt im Kontext der Nachkriegsliteratur Theodor Adornos und Max Horkheimers Verdikt gegen unterhaltsame Literatur und Erfolgsliteratur zum Tragen. Im Rahmen ihrer umfassenden Kulturkritik hatten sie einer auf die Massen ausgerichteten Literatur grundsätzlich die Qualität abgesprochen und sie als Produkte einer 'Kulturindustrie' gewertet. Der Einfluss ihrer Dialektik der Aufklärung (1944) verankerte den Begriff der 'Kulturindustrie' im deutschen Literaturbetrieb und forcierte die von Fiedler dann attackierte strikte Teilung der Literatur in zwei gegensätzliche Kategorien. ${ }^{18}$

Im Zuge seiner Auseinandersetzung mit bestehenden Qualitätshierarchien rückt Fiedler das Verhältnis zwischen Autor und Leser in den Vordergrund, das dann von Wittstock aufgegriffen wird. Fiedler wertet die Position des allgemeinen Lesers auf:

In fact, Post-Modernism implies the closing of the gap between critic and audience, too, if by critic one understands 'leader of taste' and by audience 'follower'. But most importantly of all, it implies the closing of the gap between artist and audience, or at any rate, between professional and amateur in the realm of art. ${ }^{19}$

Durch die Aufwertung des nicht professionellen Lesers gegenüber der Kritik steigert sich der Einfluss des Publikums auf die Ästhetik. Fiedler versieht die Leserschaft mit einer aktiven Rolle bei der Festlegung des Geschmacks, womit er eine Umerziehung der Kritik anstrebt, die ihren elitären Status aufgeben und sich populären Formen öffnen soll. Er führt zudem mit der Geschmacksmetapher eine zentrale Bildlichkeit der Lesbarkeitsdebatte ein, die an die viel älteren Diskussionen um den 'Geschmack' anknüpft, um sie unter Hervorhebung der Bedeutung des Amateurlesers zu

\footnotetext{
18 Vgl. Horkheimer/Adorno 2000. Eine ausführliche Analyse wird in Kapitel 2 der vorliegenden Arbeit geleistet werden.

${ }^{19}$ Fiedler 1971, S. 478.
} 
aktualisieren. ${ }^{20}$ Fiedlers Konfiguration des Autor-Leser-Verhältnisses verabschiedet die romantische Vorstellung des Autorgenies, während der Leser bei der Textgenese zusehends an Initiative gewinnt.

Neben der Rekonfiguration des Autor-Leser-Verhältnisses orientiert sich Wittstock vor allem an Fiedlers Einstellung gegenüber Unterhaltung. Mehr als zwanzig Jahre vor Wittstock fordert dieser: 'What is demanded [...] is learning to be less serious, [...], a form of entertainment. ${ }^{21}$ Fiedler bekräftigt zunächst die Dichotomie zwischen Ernst und Unterhaltung. ${ }^{22}$ Dann attackiert er die Vorrangstellung der E-Literatur und initiiert eine Aufwertung von Unterhaltung, die sich jedoch nicht als völlige Aufgabe der EPosition, sondern als dynamische Verschiebung der Prioritäten darstellt. Diese Umwertung treibt Wittstock später in Leselust programmatisch voran.

Wittstock bezieht sich in seinen theoretischen Darlegungen zur Postmoderne neben Fiedler hauptsächlich auf Umberto Eco. Ecos herausragende internationale Bedeutung als Theoretiker und Praktiker der Postmoderne - dieses Zusammenspiel ist für die Debatte um die 'Neue Deutsche Lesbarkeit' konstitutiv - machen seine theoretischen Schriften zu aufschlussreichen Anhaltspunkten für die Erschließung des postmodernen Literaturbegriffs. ${ }^{23}$ Besonders mit dem Erzählen, der Unterhaltung, der Idealvorstellung von der Leserschaft und der Breitenwirkung führt Eco Aspekte an, die für die Lesbarkeitsdebatte im Deutschland der 1990er Jahre relevant sind.

In der Postille a 'Il nome della rosa'/ Nachschrift zum Namen der Rose'(1984, in Deutschland 1986) verknüpft Eco Erzählen mit Unterhaltung:

Ich wollte den Leser unterhalten, er sollte Spaß an der Sache haben. [...] Er soll, während er sich unterhält, etwas lernen. Ob der Leser etwas über die Welt oder etwas über die Sprache lernen soll, ist eine Frage, in der die Poetiken der erzählenden Kunst divergieren, aber das ändert nichts an der Grundidee. Der Idealleser von Finnegans Wake soll sich am Ende genausogut unterhalten wie der Leser von Winnetou. Zumindest genausogut. Allerdings auf andere Weise. ${ }^{24}$

\footnotetext{
20 Zur Tradition der Geschmacksmetapher vgl. Weimar/Solms 1997.

21 Fiedler 1971, S. 466.

22 Als Beispiele des 'serious novel' nennt Fiedler die Werke von Marcel Proust, James Joyce und Thomas Mann, als Beispiel der Unterhaltungsseite u.a. Lewis Carroll, J.R.R. Tolkien oder Karl May. Die Beispiele bestätigen den universellen Charakter von Fiedlers Theorie; vgl. ebd., S. 471f.

23 Vgl. Wittstock 1995, S. 48 und 50. Erik Schilling sieht Eco in seiner Studie zu dessen Einfluss auf die deutsche Literatur in der 'Rolle eines Wegbereiters' für die Postmoderne (Schilling 2012, S. 18).

24 Eco 1986, S. 69.
} 
Eco rekurriert auf die horazische Tradition des docere et delectare, die als ebenbürtige Komponenten erscheinen. ${ }^{25}$ Beide stellt er in den Kontext des Erzählens, dem er einen Qualitätsanspruch zuordnet. Zur Definition von Unterhaltung führt er mit Joyce und May Beispiele an, die Fiedler exemplarisch für hohe und niedrige Literatur verwendet. ${ }^{26}$ Für beide Pole formuliert Eco einen Unterhaltungswunsch, deutet aber zugleich auf diverse Unterhaltungsformen bzw. auf die unterschiedlichen Unterhaltungsbedürfnisse der Leser. Unterhaltung wird zu einer inhärenten Eigenschaft von Literatur, die vom ästhetischen Anspruch eines Werkes unabhängig ist.

Eco verbindet die Kategorien des 'Ideallesers' und der Unterhaltung mit Breitenwirkung. Den Idealleser entwirft er in der Nachschrift in der Konstellation zwischen Autor, Text und Leser. ${ }^{27}$ Sobald ein Text fertig sei, so Eco, entfalte sich ein Dialog zwischen Text und Leser, aus dem sich der Autor herauszuhalten habe. Daher laufen ihm zufolge während der Arbeit zwei Dialoge ab: 'einer zwischen dem entstehenden Text und allen zuvor geschriebenen Texten (jedes Buch wird aus anderen und über andere Bücher gemacht) und einer zwischen dem Autor und seinem gedachten Wunsch-, Modell- oder Musterleser.'28 Der erste Punkt bekräftigt das postmoderne Intertextualitätsprinzip. ${ }^{29}$ Der zweite impliziert, dass der Leser indirekten Einfluss auf die Textgenese nimmt. Der Autor bleibt die aktive Instanz, jedoch bedenkt er die Bedürfnisse seiner zukünftigen Leser, was einen grundlegenden rhetorischen Aspekt einbringt. Hierbei unterscheidet Eco zwischen zwei Herangehensweisen:

In beiden Fällen heißt schreiben - ob nun der Schreibende glaubt, ein vorhandenes Publikum anzusprechen, das mit dem Geld in der Hand vor der Tür steht, oder ob er sich vornimmt, für einen künftigen Leser zu schreiben - sich mit Hilfe des eigenen Textes den gewünschten Lesertyp zu schaffen. ${ }^{30}$

Die erste Strategie steht für eine Beachtung des Publikumsgeschmacks zur Zeit des Schreibprozesses, wodurch Eco den finanziellen Erfolg als Desiderat poetologisch

\footnotetext{
$25 \mathrm{Zu}$ den rhetorischen Wirkungsabsichten vgl. Quintilian 2011, Bd. 2, XII, 59, S. 779-781.

26 Vgl. Fiedler, S. 471.

27 Vgl. Eco 1986, S. 55-60.

28 Ebd., S. 55.

29 Es handelt sich um eine Abkehr von einem avantgardistischen Originalitätsstreben und ein Spiel mit intertextuellen Referenzen, die harmonisch in den eigenen Text eingebaut werden sollen. Vgl. Lützeler 1997, insbesondere S. 119-131 und Eco 1986, S. 76-82.

30 Ebd., S. 56.
} 
legitimiert. Die zweite Strategie strebt ebenso danach, dem Leser zu gefallen, jedoch ohne finanzielles Kalkül oder unmittelbaren Erfolg in der Gegenwart. Was beide Strategien eint, ist die wirkungsästhetische Ausrichtung auf eine bereits vorhandene bzw. eine imaginäre Leserschaft. Dies verdeutlicht die poetologische Komplexität der Breitenwirkung, deren Facetten für die Lesbarkeit bedeutsam sind, da sie mit den Fragen nach der Legitimität und Qualität von Lesbarkeit unmittelbar verbunden sind.

Eco benennt Unterhaltung als Hauptmittel zur Erreichung von Breitenwirkung: 'Zweifellos findet ein Roman, wenn er unterhaltsam ist, Anklang beim Publikum.' ${ }^{11}$ Zur Unterhaltung des Lesers legt er sein besonderes Augenmerk auf die Handlung. Er macht geltend, dass die Postmoderne eine 'neue Phase der erzählenden Kunst' mit einer 'Wiederaufwertung der Handlung' initiiert habe. ${ }^{32}$ Eco verleiht den Kategorien der Unterhaltung, des Erzählens und der Handlung das Prädikat Kunst, womit er eine Reevaluierung der Qualität unterhaltsamer Literatur seit der Postmoderne unterstreicht:

Unbestreitbar hat der moderne Roman versucht, die Unterhaltung durch den dramatischen Handlungsverlauf [...] abzubauen, um dafür andere Arten von Unterhaltung zu privilegieren. Ich als großer Bewunderer der Poetik des Aristoteles bin trotz allem immer der Ansicht gewesen, daß ein Roman auch und vor allem durch seine Handlung unterhalten soll. ${ }^{33}$

Eco macht sich für Unterhaltung durch die Handlung stark. Mit einer 'Wiederentdeckung nicht nur der Handlung, sondern auch des Vergnügens', verknüpft er Handlung und Unterhaltung und erschließt mit dem Verweis auf die Poetik des Aristoteles in genau jenem Werk eine stabile theoretische Basis, das als Ausgangspunkt und anhaltend bedeutender Maßstab für literarische Qualität gilt. ${ }^{34}$ Aufgrund dieser allgemeinen Aufwertung in der Praxis stellt er in den 1980er Jahren einen qualitativen Umschwung in der Literaturlandschaft fest. ${ }^{35}$ Indem Eco Aristoteles zum Vorbild wählt, liefert er ein Indiz für die Interaktion zwischen aristotelischer Poetik und der Postmoderne. Seine postmoderne Theorie vereint demnach knapp ein Jahrzehnt vor Wittstocks Leselust den internationalen und den

\footnotetext{
31 Ebd., S. 70.

32 Vgl. ebd., S. 72; unter Handlung versteht Eco ein Spiel mit verschiedenen Einflüssen, die im Text gemischt und neukombiniert werden, aber ebenfalls als einheitliche Handlung funktionieren; vgl ebd., S. 73.

33 Ebd., S. 70.

34 Ebd, S. 76.

35 Ebd.
} 
historischen Kontext der Lesbarkeitsdebatte im Deutschland der 1990er Jahre.

Im Kontext der 1990er Jahre wird der Lesbarkeitsbegriff im Rahmen des Literaturstreits stark gemacht. Dabei erhält das Programm der 'Neuen Deutschen Lesbarkeit' eine diachronische und eine synchrone Komponente des Wettstreits, die beide produktive Energie aus der Dynamik des Wettstreits beziehen und mit dem Ziel eingesetzt werden, die ästhetische Qualität und den Publikumserfolg der deutschen Gegenwartsliteratur zu steigern. Das Programm der 'Neuen Deutschen Lesbarkeit' positioniert sich dabei in Ablehnung einer älteren, politisch engagierten Nachkriegsliteratur. Diese Opposition teilen die Befürworter der 'Neuen Deutschen Lesbarkeit' mit den Kritikern Frank Schirrmacher und Ulrich Greiner sowie mit dem Philosophen und Journalisten Karl-Heinz Bohrer. Diese treten für eine auf einem hohen ästhetischen Anspruch beruhende Literatur ein, die den Anschluss an die Moderne sucht. ${ }^{36}$ Beide Parteien engagieren sich auch hier für einen ästhetischen Umschwung, dessen Notwendigkeit sie in zwei synchronen Konkurrenzverhältnissen begründet sehen: in der Konkurrenz mit ausländischen Werken und in der Konkurrenz mit den neuen Medien. Dies löst den Wettstreit von rein literarischen Parametern und etabliert ihn im Kontext eines globalen Marktes, wie es ihn in der deutschen Literaturgeschichte bis dato nicht als poetologische Größe gegeben hat. Im Zentrum steht eine Auseinandersetzung um die Gunst des Publikums, in der die im deutschsprachigen Raum etablierten Literaturvorstellungen bei der Frage nach dem Publikumsgeschmack vermehrt in ein Spannungsverhältnis geraten und die Bedeutung von Literatur als publikumswirksames Medium in Frage gestellt wird.

Politycki situiert das Wettkampfmotiv in einem internationalen Kontext und macht 1998 einen nachhaltigen Erfolg des Programms der 'Neuen Deutschen Lesbarkeit' geltend: 'Mit dem Durchbruch der "Neuen Deutschen Lesbarkeit” meldete sich unsre Gegenwartsliteratur zurück unter die europäischen Literaturen'. ${ }^{37}$ Die Durchbruchs-Metapher suggeriert einen langwierigen jedoch erfolgreichen Kampf um die qualitative Anerkennung von Lesbarkeit. Um seine Behauptung zu legitimieren, bezieht sich Politycki auf eine theoretisch fundierte Basis: Die Wende in der deutschen

\footnotetext{
$36 \mathrm{Zu}$ deren poetologischen Vorstellungen siehe Kapitel 3.

37 Politycki 1998 f, S. 5.
} 
Literaturlandschaft sei 'ein literarischer Paradigmenwechsel' gewesen. ${ }^{38}$ Er nutzt hiermit den vom amerikanischen Wissenschaftsphilosophen Thomas S. Kuhn in The Structure of Scientific Revolutions (1962) entwickelten Paradigmenbegriff als poetologische Profilierungsstrategie. Kuhns für die Wissenschaftsgeschichte entworfene und später - wie von ihm selbst intendiert - u.a auf die Kunst angewandte Theorie fungiert bei Politycki als Prestigegarantin, um Lesbarkeit als dominante poetologische Kategorie der deutschen Gegenwartsliteratur herauszustellen. ${ }^{39}$

Kuhns Paradigmenbegriff ist für die vorliegende Arbeit hilfreich, da ihm ein Wettkampfprinzip inhärent ist. Kuhn spricht von 'controversies over fundamentals'; der Wettstreit zwischen grundlegenden Kategorien mündet dann in die Etablierung eines neuen Paradigmas: es ist '[a] universally recognized scientific achievement[...] that for a time provide[s] model problems and solutions to a community of practitioners' ${ }^{40}$ Ein Paradigma muss sich demnach seinen Rang erstreiten, um von der gesamten Gemeinschaft anerkannt zu werden und - wie die Betonung des Universalcharakters signalisiert - in einem breiten Geltungsbereich $\mathrm{zu}$ wirken. Zudem betont der dynamische Ansatz, dass ein neues Paradigma neben dem Angebot von Problemlösungen auch Fragestellungen aufwirft, die weitere Erkundungsmöglichkeiten eröffnen.

Zur Durchsetzung eines neuen Paradigmas bedarf es einer Zusammenarbeit von Befürwortern, die Kuhn mittels der folgenden Merkmale charakterisiert:

Their achievement was sufficiently unprecedented to attract an enduring group of adherents away from competing modes of scientific activity. Simultaneously, it was sufficiently open-ended to leave all sorts of problems for the redefined group of practitioners to resolve. ${ }^{41}$

Ein Paradigma muss demnach keine neue Idee verkörpern, sondern kann auf traditionellen Formen basieren, was mit Polityckis Lesbarkeitsbegriff zu vereinbaren

\footnotetext{
38 Ebd., S. 7.

39 Zur Anwendbarkeit des Begriffs auf die Künste vgl. Kuhn 1996, S. 207-210.

40 Ebd., S. X. Die Epochenschwellen-Theorie des deutschen Historikers Reinhart Koselleck etwa geht von einer chronologisch linearen Entwicklung aus, in der eine Epoche direkt, unterbrochen lediglich von einer 'Epochenschwelle' titulierten Übergangsphase, aus einer anderen hervorgeht.; vgl. u.a. Koselleck 1973 und Herzog/Koselleck 1987.

41 Kuhn 1996, S. 10.
} 
ist. ${ }^{42}$ Wenn Politycki geltend macht, Lesbarkeit sei 'uralt' und es habe sie 'in allen Jahrhunderten aller literarischen Entwicklungen immer wieder einmal gegeben', so verdeutlicht er, dass es sich bei dem Programm der 'Neuen Deutschen Lesbarkeit' um die besondere Profilierung einer gängigen Kategorie handelt. ${ }^{43}$ Zudem muss ein Paradigma auch von einigen der einstigen Widersacher anerkannt werden, um seinen Status zu bestätigen. Dabei braucht ein Paradigma bei dessen Einführung nicht theoretisch ausgereift zu sein. Vielmehr sieht Kuhn es als positive Begleiterscheinung eines Paradigmenwechsels an, dass er ein umfassendes Erkundungsspotenzial eröffnet.

Tatsächlich scheint die unmittelbare Eingängigkeit des Begriffs 'Lesbarkeit' bewirkt zu haben, dass seitens der Germanistik eine fundierte theoretische Untersuchung seiner Bedeutung, Tradition, theoretischen Parameter und praktischen Umsetzung bislang ausgeblieben ist. Zwar verwenden die sporadischen akademischen Arbeiten zu diesem Umfeld den Begriff 'Lesbarkeit', was auf dessen grundsätzliche Konsensfähigkeit hindeutet, jedoch sehen sie den Begriff überwiegend eher als oberflächliche oder opportunistische literaturpolitische Strategie, ohne sich intensiv mit Lesbarkeit als poetologischer Kategorie auseinanderzusetzen. Dabei steht vor allem ein Misstrauen gegenüber der Lesbarkeit im Vordergrund, das in einer traditionsreichen Entgegensetzung von wirkungsorientierter Strategie und literarischer Qualität gründet. ${ }^{44}$ So stellt Rachel Halversons Essay 'The New German Lesbarkeit in Action' (2010) die Frage, ob Lesbarkeit zwangsläufig zu einem Kompromiss hinsichtlich literarischer Qualität führen müsse. ${ }^{45}$ Lesbarkeit sieht Halverson als dynamisches Zusammenspiel zwischen Sprache und Handlung, mit dem strategisch sowohl die Kritiker als auch eine breite Masse angesprochen werden sollen. Katharina Hall geht in 'The Author, the Novel, the Reader and the Perils of "Neue Lesbarkeit" (2006) ebenso auf die Qualitätsfrage ein, unterscheidet jedoch im Sinne von Fiedlers postmoderner Theorie zwischen Elementen der 'low' und der 'high culture' in den Romanen Bernhard Schlinks, wobei sie auf Sujet (Holocaust als Hochkultur) und Genre (Liebes- bzw.

\footnotetext{
42 An anderer Stelle äußert Kuhn: 'Even the project whose goal is paradigm articulation does not aim at unexpected novelty' (Ebd., S. 35).

43 Vgl. Fußnote 5.

44 Auch hier lassen sich wie bei der Privilegierung der E-Literatur deutlich idealistische Vorgaben ausmachen. Die Ablehnung einer wirkungsorientierten Literatur im Idealismus wird in Kapitel 2 am Beispiel von Georg Wilhelm Friedrich Hegels Vorlesungen über die Ästhetik (1817-1829) veranschaulicht werden.

45 Halverson 2010.
} 
Kriminalgeschichte als Populärkultur) eingeht. Dabei bewertet sie die popkulturellen Aspekte kritisch und hinterfragt deren literarischen Wert. ${ }^{46}$ Stuart Taberner wiederum betont in seinem Essay 'A New Modernism or "Neue Lesbarkeit"? Hybridity in Georg Klein's Libidissi' (2002) die Bedeutung der Lesbarkeit für den Erfolg der deutschsprachigen Literatur im internationalen Markt, wobei Lesbarkeit hier in der Form einer Mischung aus hoch- und popkulturellen Elementen in Erscheinung tritt. ${ }^{47}$ Die Signifikanz von Lesbarkeit im Prozess literarischer Globalisierung hebt auch Anke S. Biendarra in ihrer Monographie Germans Going Global (2012) hervor. ${ }^{48}$ Bei diesen Beiträgen ist auffällig, dass sie alle aus dem englischsprachigen Ausland stammen, das eher dazu tendiert 'readability' als literarische Größe anzuerkennen als die deutsche Germanistik. Als germanistisch fokussierte Ansätze teilen sie das Misstrauen gegenüber Strategien, die der Breitenwirkung dienen, aber sie setzen sich immerhin mit dem Phänomen auseinander und erkennen die Bedeutung des Programms der 'Neuen Deutschen Lesbarkeit', ohne jedoch den Lesbarkeitsbegriff in seiner theoretischen, historischen und praktischen Komplexität greifbar zu machen.

Die vorliegende Arbeit wird den vom Programm der 'Neuen Deutschen Lesbarkeit' an einem brisanten kulturellen Wendepunkt gegebenen Impuls ernst nehmen und Lesbarkeit als grundlegende poetologische Kategorie sicht- und analysierbar machen. Dazu werden zunächst poetologische Parameter erschlossen werden, die für die Lesbarkeit eines Textes wirksam sind, ehe dann anhand einer Reihe von Werken aus dem frühen 21. Jahrhundert veranschaulicht werden wird, wie Autoren diese Parameter anwenden, um teils komplexe Sujets für eine diverse Leserschaft zugänglich zu vermitteln. Es soll gezeigt werden, dass dem Begriff 'Lesbarkeit' gerade im deutschen Kontext ein immenses Potenzial innewohnt, das von Autoren insbesondere in konkreten Konfliktsituationen poetologisch ausgeschöpft wird. Dies geschieht zu dem Zweck, den Kanon von vermeintlich 'hoher' Literatur um Werke zu erweitern, die zuvorderst auf Lesbarkeit zielen. Dabei soll die bislang von der Forschung kaum wahrgenommene Komplexität des Lesbarkeitsbegriffs herausgearbeitet werden, die sich vor allem aus der

\footnotetext{
46 Hall 2006.

47 Taberner 2002 und Biendarra 2012.

48 Biendarra verweist an vier Stellen sporadisch auf die 'Neue Deutsche Lesbarkeit', vgl. Biendarra 2012, S. 15, 69, 72 und 83 .
} 
Dynamik des Wechselspiels zwischen Theorie, Historie und Praxis ergibt. In diesem Wechselspiel liegt die Kapitelfolge der vorliegenden Arbeit begründet. ${ }^{49}$ Die ersten drei Kapitel befassen sich zunächst einerseits mit der Theorie (Kapitel 1) und andererseits mit zeitgeschichtlichen Entwicklungen, in denen Lesbarkeit als poetologische Kategorie Bedeutung erlangte, wobei die Tradition der Auseinandersetzung mit Lesbarkeit erörtert wird (Kapitel 2) und dann spezifisch die Debatte um Lesbarkeit im Kontext des Literaturstreits verfolgt wird (Kapitel 3). Dadurch wird eine theoretische und historische Basis für zwei Kapitel geschaffen, in denen das produktive Zusammenspiel von Theorie und Praxis untersucht werden soll (Kapitel 4 und 5). Hier wird zugleich verdeutlicht werden, dass das Eintreten für Lesbarkeit seit den 1990er Jahren tiefgreifende Veränderungen in der deutschen Literaturlandschaft bewirkt hat.

Kapitel 1 entwirft ein theoretisches Instrumentarium, das eine fundierte Auseinandersetzung mit Lesbarkeit ermöglicht. Die hier behandelten poetologischen Phänomene werden zur klassischen Rhetorik in Bezug gesetzt, wobei vor allem die grundlegenden rhetorischen Werke von Aristoteles und Quintilian berücksichtigt werden. Zudem liefert die Poetik des Aristoteles wichtige Anhaltspunkte hinsichtlich der Handlung; die Arbeiten von Cicero und Horaz hingegen dienen als Grundlage für die rhetorischen Wirkungsabsichten docere, movere und delectare. Diese Aspekte werden dann im Essay 'Mr Difficult' (2002) des germanophilen amerikanischen Autors Jonathan Franzen weiterverfolgt, um eine internationale Perspektive für die Auseinandersetzung mit Lesbarkeit zu erhalten. In seinem Essay setzt sich Franzen dezidiert mit Lesbarkeit auseinander, die er als grundlegende Bedingung im 'Vertrag' zwischen Autor und Leser versteht. Dieser Vertrag verpflichtet den Autor dazu, das Unterhaltungsbedürfnis des Lesers ernst zu nehmen. Franzen liefert ein Beispiel für eine ergiebige, auf Lesbarkeit gründende Autorpoetik des frühen 21. Jahrhunderts, die als Referenzpunkt für die nachfolgenden Kapitel fungiert. Herangezogen wird auch die Narratologie, um diese an sich unterschiedlichen theoretischen Grundlagen zu situieren und die Relevanz sowie die Art und Weise ihres Zusammenspiel für den

\footnotetext{
49 Während Lesbarkeit an sich ein theoretisch unbelichteter Begriff ist, sind zahlreiche zu besprechende Autoren und Epochen ausgiebig bearbeitet. Die Relevanz der zitierten Sekundärliteratur für Lesbarkeit wird zu gegebener Zeit etabliert werden.
} 
Lesbarkeitsbegriff zu bestimmen.

Kapitel 2 verortet Lesbarkeit in der Tradition deutscher Erzählliteratur, wobei punktuell der jeweilige Stellenwert von Lesbarkeit herausgearbeitet werden soll. Es wird aufgezeigt, dass inhärente Aspekte der Lesbarkeitsebatte - insbesondere die binäre Unterscheidung zwischen 'hoher Kunst' und 'niedriger Trivialliteratur' - bereits um 1700 in Deutschland präsent sind. Dies ist ein Gegensatz, der in der deutschen Literaturgeschichte häufig zu Lesbarkeit in Bezug gebracht wird und zum Misstrauen gegenüber Lesbarkeit führt. Weitere darzustellende Schlüsselmomente sind die Debatte um die narrative Literatur in der zweiten Hälfte des 19. Jahrhunderts sowie die vermeintliche Privilegierung von 'schwieriger' Avantgarde-Literatur in der Moderne. Das historische Kapitel wird mit der Betrachtung der didaktisch motivierten Autoren in Ost- und West-Deutschland während der Nachkriegszeit enden, in der die zentralen Streitpunkte der in den frühen 1990er Jahren entstehenden Debatte angelegt sind.

In Kapitel 3 soll die Bedeutung der Lesbarkeit als Referenzpunkt im Kontext eines globalen Marktes nach der Wiedervereinigung untersucht werden. Dazu analysiert das Kapitel die poetologischen und politischen Ansätze von Wittstock und Politycki, die zur Mitte der 1990er Jahre als Lösungsansätze dafür formuliert wurden, der deutschen Literatur den Erfolg bei einer nationalen und internationalen Leserschaft zu sichern. Diese sollen nicht nur mit den Lesbarkeitsformen der Tradition verglichen, sondern auch zueinander in Bezug gesetzt werden, um die Divergenzen zwischen den Standpunkten zu erfassen. Zudem dient die Kurzgeschichtenanthologie Wenn der Kater kommt (1996) des Journalisten und Herausgebers Martin Hielscher als Gradmesser für den frühen Erfolg von Wittstocks und Polityckis poetologischen Forderungen. Auf die bewusst vorangetriebene Kulturpolitik und die Machtspiele zwischen den Generationen, die hierdurch besonders zur Schau kommen, wird auch eingegangen. Dadurch soll ein umfassendes Bild von Lesbarkeit gezeichnet werden, das als Ausgangspunkt für die Erkundung der Entwicklungen im frühen 21. Jahrhundert fungieren wird.

In den Kapiteln 4 und 5 wird es darum gehen, auch die literarische Praxis von Lesbarkeit $\mathrm{zu}$ erschließen. Es soll dabei herausgearbeitet werden, mit welchen Strategien auf Lesbarkeit zielende Poetologien im deutschen Literaturbetrieb etabliert wurden, wie die Theorie in die Praxis Eingang findet und wie die fiktionalen Werke von 
der literarischen Welt aufgenommen wurden.

In Kapitel 4 fungiert die Kollaboration der Autoren Helmut Krausser (geb. 1964), Daniel Kehlmann (geb. 1975) und Thomas Glavinic (geb. 1972) als konkretes Beispiel für eine Zusammenarbeit zur Etablierung von Lesbarkeit als Paradigma der deutschen Gegenwartsliteratur um 2000. Krausser, der von der Kritik bereits in den 1990er Jahren im Kontext der von Wittstock angestoßenen Entwicklungen rezipiert wurde, initiierte die Kooperation mit Kehlmann, um einen literarisch gleichgesinnten Partner zu fördern und damit den Einfluss ihrer grundsätzlich übereinstimmenden poetologischen Ansichten zu steigern. Dieselbe Vorgehensweise wandte Kehlmann später bei Glavinic an. Zunächst stehen die Strategien im Vordergrund, mit denen diese Autoren ihre Literaturprogramme in den Literaturbetrieb einführten. ${ }^{50}$ Die anschließende Analyse der Reaktionen in der Kritik und der deutschen Literaturwissenschaft soll zeigen, dass die Autoren, indem sie sowohl auf ein Fachpublikum als auch auf die allgemeine Öffentlichkeit zielten, eine umfassende Reevaluierung von literarischem Wert in Angriff nahmen. Das Ende des Kapitels befasst sich mit Kraussers Nicht ganz schlechte Menschen (2012), Kehlmanns Die Vermessung der Welt (2005) und Glavinics Das größere Wunder (2013) und untersucht exemplarisch die praktische Umsetzung von Lesbarkeit im Erzählwerk.

In Kapitel 5 dienen die poetologischen Schriften von Ulrike Draesner (geb. 1962) und Felicitas Hoppe (geb. 1960) als kritische Gegenstimmen zu den in den 1990er Jahren durch das Programm der 'Neuen Deutschen Lesbarkeit' angestoßenen Entwicklungen. Zum einen verdeutlichen ihre Vorbehalte die Streitpunkte der Lesbarkeitsdebatte im frühen 21. Jahrhundert. Zum anderen bestätigen die theoretischen und fiktionalen Werke der beiden Autorinnen Lesbarkeit als zentrale Kategorie der deutschen Literatur um die Jahrtausendwende. Denn die Analysen von Hoppes Kinderbuch Iwein Löwenritter (2008) und ihrem Roman Hoppe (2012) sowie von Draesners Roman Sieben Sprünge vom Rand der Welt (2014) werden aufzeigen, dass die Autorinnen Lesbarkeit als Desiderat keineswegs kategorisch ablehnen, sondern dass sie

\footnotetext{
50 Dies ist ein elementarer Bestandteil von Kuhns Analysemodell eines Paradigmenwechsels: 'we shall have to examine $[\ldots]$ the techniques of persuasive argumentation within the quite special groups that constitute the community' (Kuhn 1996, S. 94).
} 
sich kreativ mit dem Potenzial des Lesbarkeitsbegriffs auseinandersetzen und dadurch einen weiteren wichtigen Beitrag zu seiner Umsetzung in die Praxis der deutschen Gegenwartsliteratur leisten.

Zum Abschluss sollen die Ergebnisse der vorhergehenden Kapitel zusammengeführt werden und einer Erkundung der Zukunftsperspektiven von Lesbarkeit in der deutschen Literatur dienen. Die Entwicklungen seit 1990 werden danach befragt werden, welche Grundlagen, Anregungen und Herausforderungen sie der Generation nach Krausser, Kehlmann und Glavinic hinterlassen haben. Um auch diese Frage anhand eines konkreten Beispiels von der Praxis her $\mathrm{zu}$ untersuchen, wird abschließend die Autorpoetik des Autors einer jüngeren Generation, Benedict Wells (geb. 1984), hinsichtlich der Bedeutung von Lesbarkeit für seine Literaturauffassung befragt. Dies soll dazu dienen, die Nachhaltigkeit der Lesbarkeit zu erkunden und festzustellen, inwiefern sie sich in der deutschsprachigen Literatur der Gegenwart als literarisches Grundprinzip etabliert hat. 


\section{Kapitel 1: Lesbarkeit als theoretisches Prinzip und der Vertrag zwischen Autor und Leser}

Während die anderen Kapitel Lesbarkeit im dynamischen Zusammenspiel von Theorie, Historie und Praxis analysieren, widmet sich das vorliegende Kapitel spezifisch den theoretischen Aspekten der Lesbarkeit. Auch wenn sich Lesbarkeit als Begriff und literarisches Grundprinzip erst in dieser Wechselwirkung konstituiert, ist es unerlässlich, theoretisch fundierte Kategorien herauszuarbeiten, die im weiteren Verlauf der vorliegenden Arbeit als Orientierungspunkte dienen und eine systematische Analyse von Lesbarkeit ermöglichen können. In der Einleitung wurde bereits erörtert, dass sich der um 1990 diskutierte Begriff der Lesbarkeit aus klassischen Vorbildern speist, was besonders an der Betonung des Publikumsbezugs und der Unterhaltung durch die Handlung deutlich wurde. Für diese beiden Kategorien dienen die klassische Rhetorik sowie die Poetik des Aristoteles als Referenzsysteme. Demnach handelt es sich bei Lesbarkeit um ein Prinzip, das sowohl aus der philosophischen als auch aus der rhetorischen Tradition schöpft. ${ }^{51}$ Darüber hinaus ist es signifikant, dass Uwe Wittstock und Matthias Politycki die Wirksamkeit der anglo-amerikanischen Literatur zur Frage der Lesbarkeit deutscher Literatur in Verbindung bringen. Dem entspricht, dass auch Helmut Krausser, Daniel Kehlmann und Thomas Glavinic besonders die Werke des amerikanischen Autors Jonathan Franzen als einflussreiche Vorbilder für Lesbarkeit verstehen. Für die Theorie der Lesbarkeit ist Franzen deswegen relevant, weil er sich in seiner Autorpoetik - als paradigmatisches Beispiel kann sein 2002 im New Yorker erschienener Essay 'Mr. Difficult' gelten - intensiv mit den theoretischen Aspekten von Lesbarkeit auseinandersetzt. Aus dieser Konstellation ergibt sich folgende Problematik: Aristoteles' Ausführungen beziehen sich vorrangig auf die Gattung des Dramas, die Rhetorik liefert eine auf den mündlichen Vortrag fokussierte Theorie, während in der Gegenwart die Erzählliteratur, insbesondere der Roman, hinsichtlich der für die Debatte um die Lesbarkeit zentralen Fragen im Zentrum steht, wobei der Roman typischerweise vom Rezipienten still und alleine gelesen wird. Um die Komplexität dieser Problemstellung angemessen darzustellen und die Produktivität der daraus entstehenden

\footnotetext{
51 Zu Dichtung und Philosophie vgl. Aristoteles 1994, 9, S. 29.
} 
Spannungen zu verdeutlichen, ist es sinnvoll, die theoretischen Komponenten zunächst zum gegenwärtigen Forschungsfeld der Narratologie in Bezug zu setzen.

Die zeitgenössische Narratologie greift vor allem bei der Handlung auf Aristoteles' Poetik zurück. Monika Fludernik und Greta Olson bezeichnen Aristoteles in ihrer Einführung zu Olsons Sammelband Current Trends in Narratology (2011) als 'first narratologist'. ${ }^{52}$ In ihrer Introduction to Narratology (2009) nutzt Fludernik insbesondere die Dramentheorie für die Ausführungen zur Handlung, 'since the analysis of plot in the novel and the treatment of plot in drama are very largely in agreement. ${ }^{53}$ Brian Richardson äußert in seinem Beitrag zum Drama in David Hermans Cambridge Companion to Narrative (2007):

From the outset, theories of drama and theories of narrative have been closely linked. Aristotle's Poetics, still the starting-point for any narrative theory, devotes more space to drama than to epic. The topics he covers, including character, plot, beginnings and endings [...] are as relevant to narrative theory as to a poetics of drama. ${ }^{54}$

Richardson legitimiert somit das Rekurrieren der Narratologie auf die Dramentheorie von Aristoteles, der er einen höheren Status als Aristoteles' Theorie zum Epos einräumt. Aus der aristotelischen Dramentheorie sind für die narratologische Forschung hauptsächlich die theoretischen Prinzipien der Handlungsführung relevant. Die von Richardson angeführten Handlungsprinzipien werden für die Analyse von Lesbarkeit von zentraler Bedeutung sein.

Irene De Jong wiederum hebt in Narratology and Classics (2014), ihrer Studie zur Narratologie antiker Texte, die Relevanz der Rhetorik für die Narratologie hervor. Sie erkennt Kongruenzen zwischen der modernen Narratologie und den antiken Vorbildern: 'A possible explanation for the success of narratology within the field of classics is that its terms resemble those of rhetoric, which has been of old the framework within which ancient literary texts are analysed.' ${ }^{55}$ Die Tatsache, dass Dichtkunst eine sprachliche Kunst ist, legitimiert den Einsatz der klassischen Rhetorik für die Analyse von Lesbarkeit als poetologischem Prinzip der Erzählliteratur. Vor allem die Analyse der

\footnotetext{
52 Fludernik/Olson 2011, S. 17.

53 Fludernik 2009, S. 5.

54 Richardson 2007, S. 142.

55 De Jong 2014, S. 9.
} 
Wirkung und des Publikumsbezugs machen es sinnvoll, die Perspektive der Rhetorik sowie auch deren terminologisches Instrumentarium einzubeziehen. ${ }^{56}$

Die aristotelische Dramentheorie und die Rhetorik treten vor allem im Rahmen einer 'postclassical narratology' in den Vordergrund. ${ }^{57}$ In der Einführung zu seinem Cambridge Companion to Narrative (2007) benennt David Herman den Einbezug der beiden in die Narratologie als wichtigen Bestandteil der 'postclassical narratology', der neue Forschungsfelder eröffnen und so das Spektrum der Narratologie erweitern könnte. ${ }^{58}$ Fludernik und Olson erkennen in der Einführung zu Current Trends in Narratology zwei Tendenzen: zum einen machen sie geltend, dass sich eine Sparte der Narratologie auf innovative Weise klassischen narratologischen Modellen nähert. Zum anderen fassen sie unter dem Begriff der 'postclassical narratology' Strömungen zusammen, die versuchen, die narratologische Theorie auf andere Bezugsfelder anzuwenden. ${ }^{59}$ Die vorliegende Arbeit wird der narratologischen Forschung mit Lesbarkeit ein bislang kaum erschlossenes Prinzip hinzufügen, das sich aus der aristotelischen Dramentheorie und der Rhetorik speist, und dadurch einen wichtigen Beitrag zur Narratologie leisten.

Im frühen 21. Jahrhundert machen Fludernik und Olson drei verschiedene Forschungsströmungen aus, die laut den Autorinnen die Zukunft der Narratologie maßgeblich beeinflussen könnten:

They are cognitive narratology, transmedial/interdisciplinary approaches, and research that takes its point of departure from an engagement with specific local and national inflections of narrative theory. [...] Such 'post' narratologies depart from the classical emphasis on prose literary texts and its disavowal of contextual and ideological issues [...]. The third type [...] explores local histories of narratological development. As will be shown, such historical work may lead to a challenge of traditional categories just as profound as that being performed in already widely acknowledged postclassical trends. ${ }^{60}$

\footnotetext{
56 James Phelan definiert in Hermans Sammelband die Bedeutung der Wirkungsausrichtung der Rhetorik auf die Narratologie anhand der Beziehung zwischen 'speaker, text, and audience. The approach postulates a recursive relationship among authorial agency, textual phenomena (including intertextual relations), and reader response. Texts are designed by authors in order to affect readers in particular ways; those designs are conveyed through words, techniques, structures, forms, and intertextual relations of texts; and reader responses are a function of and, thus, a guide to how authorial designs are created through textual phenomena.' (Phelan 2007, S. 209).

57 Den Begriff der 'postclassical narratology' prägte David Herman 1997 in Abgrenzung von der Narratologie des Strukturalismus. Vgl. Herman 1997 und Herman 1999.

58 Herman 2007 b, S. 16.

59 Vgl. Fludernik/Olson 2011, S. 2 f.

${ }^{60}$ Fludernik/Olson 2011, S. 3.
} 
Eine kognitive Analyse von Lesbarkeit ebenso wie die Anwendung auf andere Gattungen und Genres außer dem Roman sind als Desiderat vielversprechend, würden jedoch den Rahmen der vorliegenden Arbeit sprengen. ${ }^{61}$ Diese erfüllt die folgenden von Fludernik und Olson angeführten Funktionen: Die Anlage von Lesbarkeit als Zusammenspiel von Theorie, Historie und Praxis bezieht sowohl den jeweiligen zeitgenössischen als auch den historischen Kontext mit ein, was in den folgenden Kapiteln geschehen wird. ${ }^{62}$ Dass bei der theoretischen Grundlegung von Lesbarkeit jedoch auch andere Gattungen als die erzählende Prosa eine Rolle spielen, zeigt die wiederum mit der 'postclassical narratology' in Einklang stehende Bezugnahme auf die aristotelische Mimesistradition und die Rhetorik. ${ }^{63}$

Lesbarkeit ist ein komplexes Phänomen, das sich aus dem dynamischen Zusammenspiel vieler Faktoren konstituiert. Dieser Komplexität trägt Marie-Laure Ryans Versuch einer Definition von Narratologie in Hermans Cambridge Companion Rechnung. Ryan wendet sich gegen eine strikte begriffliche Festlegung:

Rather than regarding narrativity as a strictly binary feature, that is, as a property that a given text either has or doesn't have, the definition proposed below presents narrative texts as a fuzzy set allowing variable degrees of membership, but centered on prototypical cases that everybody recognizes $[\ldots]{ }^{64}$

In Entsprechung zu Ryans Ansatz bezüglich der Narratologie soll auch Lesbarkeit nicht als statische Kategorie angesehen werden, die es ermöglichen würde, gewisse Werke schlicht als lesbar oder unlesbar einzustufen. Es sollen vielmehr Parameter aus der klassischen Rhetorik und Poetik sowie aus der Poetologie der Erzählliteratur des frühen 21. Jahrhunderts eingeführt werden, die als verlässliche Bezugsfelder fungieren: Die

\footnotetext{
61 Als Beispiel für einen kognitiven Ansatz kann Troscianko 2014 gelten.

62 Die Analyse von Lesbarkeit in der vorliegenden Arbeit erfolgt im Sinne des Analysevorschlags aus Fluderniks grundlegendem Essay 'The Diachronization of Narratology' (2003): '[It will] be interesting to find out when certain techniques or constellations were first used, or when they became current and, even later, predominant, but also it will be exciting to take up the question of refunctionalisation. Do certain features and techniques acquire a different function at crucial points of the restructuring process of the narrative paradigm?' (Fludernik 2003, S. 334).

${ }^{63}$ Generell zeigt sich die Narratologie des frühen 21. Jahrhunderts äußerst inklusiv. Marie-Laure Ryan liefert folgende Definition von Narratologie: 'In summary: if narrative is a discourse that conveys story [...] and if this discourse can be put to a variety of different uses, none of them constitutive of narrativity, then its definition should focus on story. As a mental representation, story is not tied to any particular medium, and it is independent of the distinction between fiction and non-fiction. A definition of narrative should therefore work for different media (though admittedly media do widely differ in their storytelling abilities), and it should not privilege literary forms.' (Ryan 2007, S. 26). Jan Alber und Monika Fludernik machen in ihrem Sammelband zur Eruierung der 'postclassical narratology' zudem geltend, dass die Narratologie zum Ende des ersten Jahrzehnts des 21. Jahrhunderts zusehends versucht, die unterschiedlichen Anwendungsfelder zueinander in Bezug zu setzen. Vgl. Alber/Fludernik 2010, S. 4f.

64 Ryan 2007, S. 28.
} 
aristotelische Mimesistradition bietet eine wichtige Basis für die Erörterung von Handlungskategorien, während die Rhetorik als Referenzsystem für die sprachliche Gestaltung dient. Insbesondere werden dabei auch die rhetorischen Wirkungsfunktionen des docere, delectare und movere wie sie etwa Cicero in De oratore und im Orator darlegt sowie deren Anwendung auf die Dichtkunst durch Horaz in der Ars poetica von Bedeutung sein. Innerhalb dieser Bezugsfelder lässt sich ein dynamisches, variantenreiches Wechselspiel verfolgen, das eine individuelle Praxisanalyse von Werken nötig macht, um die unterschiedlichen Manifestationen von Lesbarkeit zu erkunden. ${ }^{65}$ Während Ryan Erzählen und Verständnis als weitgehend voneinander unabhängig ansieht - 'jugements of narrativity are variable, and [...] they are not crucial to understanding' - wird die vorliegende Arbeit eruieren, inwiefern ein Rückgriff auf das klassische Erzählen die Lesbarkeit eines Textes beeinflussen kann. ${ }^{66}$ Die bedeutenden und seit der Antike etablierten Parameter von Lesbarkeit sollen zunächst aus der Poetik und aus der Rhetorik abgeleitet werden. Für die Poetik dient das gleichnamige Werk von Aristoteles als Vorbild, für die Rhetorik überwiegend Quintilians umfassendes Regelwerk der Institutio Oratoria. Am Beispiel von Franzen soll dann analysiert werden, in welcher Form diese Parameter in den Lesbarkeitsdiskurs im frühen 21. Jahrhundert eingegangen sind.

Aristoteles verbindet in seiner Poetik die Darstellungsmittel mit dem Inhalt. Speziell geht es ihm um die Handlung, die er als 'Zusammenfügung der Geschehnisse' und als das 'erste und wichtigste' Gestaltungsmittel des Dramas konstituiert. ${ }^{67}$ Er etabliert folgende Grundregelung für die Umsetzung seines Mimesisbegriffs: 'es [ist] nicht Aufgabe des Dichters [...] mitzuteilen, was wirklich geschehen ist, sondern vielmehr, was geschehen könnte, d.h. das nach den Regeln der Wahrscheinlichkeit oder Notwendigkeit Mögliche. ${ }^{98}$ Die 'Wahrscheinlichkeit' nimmt gegenüber der Wirklichkeit eine privilegierte Position ein. Die Notwendigkeit steht für die innere Folgerichtigkeit der Handlung und kann somit als kompositorische Prämisse gelten.

\footnotetext{
65 Anklänge an eine solche Dynamik finden sich auch ebd., S. 31.

66 Ebd.

67 Aristoteles 1994, 24, S. 83.

68 Ebd., 9, S. 29.
} 
Die ideale Handlungskomposition, die auch die oben zitierten narratologischen Arbeiten als Ausgangspunkt ihrer Forschung zur Handlung wählen, definiert Aristoteles folgendermaßen:

die Tragödie [ist] die Nachahmung einer in sich geschlossenen und ganzen Handlung, die eine bestimmte Größe hat; es gibt ja auch etwas Ganzes ohne nennenswerte Größe. Ein Ganzes ist, was Anfang, Mitte und Ende hat. Ein Anfang ist, was selbst nicht mit Notwendigkeit auf etwas anderes folgt, nach dem jedoch natürlicherweise etwas anderes eintritt oder entsteht. Ein Ende ist umgekehrt, was selbst natürlicherweise auf etwas anderes folgt, und zwar notwendigerweise oder in der Regel, während nach ihm nichts anderes mehr eintritt. Eine Mitte ist, was sowohl selbst auf etwas anderes folgt, als auch etwas anderes nach sich zieht. Demzufolge dürfen Handlungen, wenn sie gut zusammengefügt sein sollen, nicht an beliebiger Stelle einsetzen noch an beliebiger Stelle enden, sondern sie müssen sich an die genannten Grundsätze halten. ${ }^{69}$

Legt man der Lesbarkeit das aristotelische Handlungskonstrukt zugrunde, so ergibt sich die Idealform einer durch die Adjektive 'geschlossen' und 'ganz' angedeuteten, einheitlichen Konzeption, die in Form einer lückenlosen und plausiblen Zusammenfügung der einzelnen Handlungsabschnitte auftritt. Aristoteles teilt dem Anfangs- und Endpunkt der Handlung sowie auch der Einbettung der letzteren in die Gesamthandlung eine wichtige Funktion zu, die es bei den später zu besprechenden Textbeispielen besonders zu beachten gilt, weil das Prinzip der Größe eine angemessene Ausdehnung der Handlung einfordert. Zudem führt Aristoteles für die Handlung unterschiedliche Komplexitätsstufen ein, die er mit 'einfach' und 'kompliziert' benennt. ${ }^{70}$ Die Unterscheidung beruht auf der Aussparung bzw. Verwendung von Anagnorisis und Peripetie bei der Ausgestaltung der Wende. Während Aristoteles die Schwierigkeitsgrade der Handlungsgestaltung eindeutig in einfache und komplexe Abstufungen unterteilt, wird in der vorliegenden Arbeit bei der Eruierung von Lesbarkeit - dem dynamischen Charakter des Ansatzes entsprechend - besonders das Wechselspiel einfacher und komplexer Elemente in den Fokus rücken.

Eine Kategorie dieses Wechselspiels führt Aristoteles in Bezug auf die Chronologie der Handlungsführung ein: 'Im Epos [...], das ja Erzählung ist, kann man [...] mehrere Handlungsabschnitte bringen, die sich gleichzeitig vollziehen'; diese Abschnitte müssen

\footnotetext{
69 Ebd., 7, S. 25.
}

70 Ebd., 10, S. 33. 
derart eingearbeitet werden, dass sie 'mit der Haupthandlung zusammenhängen'. ${ }^{71}$ Aristoteles hebt die Möglichkeit der Epos-Handlung hervor, mit der Chronologie brechen zu können, was als Mittel gegen Gleichförmigkeit und für mehr Spielraum bei der Textgestaltung gelten kann. Zugleich handelt es sich um eine komplexe Gestaltung, die jedoch nur dann zulässig ist, wenn die größere Komplexität der Haupthandlung und der erzählerischen Einheit des Werkes dient. Andererseits kritisiert Aristoteles eine chronologische Abfolge, wenn sich aus ihr kein einheitliches Ziel ergibt. ${ }^{72}$ Die leichter zugängliche, strikt chronologische Handlungsabfolge darf nur dann angewandt werden, wenn sie die Handlung konsequent zu einem notwendigen Ende führt. Hinsichtlich der komplexeren nicht-chronologischen Gestaltung muss die Markierung der Übergänge zwischen Neben- und Haupthandlung beachtet werden, um die Komplexität nicht unangemessen zu steigern. Hier zeichnet sich ein vitaler Prozess ab, der deutlicher als Aristoteles' Komplexitätsstufen der Handlung die Dynamik von Lesbarkeit spiegelt und bei der praktischen Umsetzung großen Freiraum lässt.

Neben der Einheit der Zeit und der Handlung wurde in der aristotelischen Tradition, vor allem im Hinblick auf das Theater, die Einheit des Ortes als nach Möglichkeit vollständiger Verzicht auf Ortswechsel rezipiert, was sich allerdings bei Aristoteles nicht findet. ${ }^{73}$ Allein aufgrund der unterschiedlichen Rezeptionsweisen ist der Roman nicht in dem Maße wie das Drama an einen Schauplatz gebunden, bei dem ein Ortswechsel produktionstechnische Schwierigkeiten nach sich zieht. Wie später aus Franzens poetologischen Äußerungen hervorgehen wird, sind für Lesbarkeit die eindeutige Kennzeichnung von Ortswechseln und deren Funktion von Bedeutung. Zudem kann die narratologische Darstellung der Räume, also deren Anschaulichkeit, als wichtiger Analysepunkt gelten. Zuletzt muss auch eine etwaige Semantisierung von Räumen berücksichtig werden, insbesondere, wenn sie dazu beiträgt, die Einheit der Handlung zu fördern und die Handlung zu einem Ziel zu führen.

Bei Aristoteles wirken die Figuren als handlungsvorantreibende Elemente, die dem größeren Ziel einer leicht zu folgenden schlüssigen Handlungsführung untergeordnet

\footnotetext{
71 Ebd., 24, S. 81. Dass für die Narratologie Aristoteles Äußerungen zum Epos ebenso relevant sind wie die zum Drama, zeigt Halliwell 2014.

72 Vgl. Aristoteles 1994, 23, S. 77.

73 Vgl. Schulz 1997.
} 
sind. ${ }^{74}$ Daher müssen auch die Figuren die Anforderungen der Wahrscheinlichkeit und der Notwendigkeit erfüllen:

\begin{abstract}
Man muß auch bei den Charakteren - wie bei der Zusammenfügung der Geschehnisse - stets [...] darauf [bedacht sein], daß es notwendig oder wahrscheinlich ist, daß eine derartige Person derartiges sagt oder tut, und daß das eine mit Notwendigkeit oder Wahrscheinlichkeit auf das andere folgt. ${ }^{75}$
\end{abstract}

Aristoteles spiegelt die konsequent logische Umsetzung der Handlungsführung auf der Figurenebene. Die Plausibilität der Figuren sowie deren Funktion, die Handlung stringent voranzutreiben, sollen dem Rezipienten den Zugang zum Text erleichtern. Zudem betont Aristoteles, dass die Figur dem Rezipienten ähnlich sein soll. ${ }^{76}$ Die Ähnlichkeit zwischen Charakter und Zuschauer kann dahingehend interpretiert werden, dass sie dem Rezipienten ein hohes Identifikationspotenzial bietet, was einerseits zu einem Interesse an der Figur führt und andererseits die Augenscheinlichkeit fördert.

In Aristoteles' Poetik zeichnen sich dynamische Prozesse ab, die sich aus Elementen des Einfachen und des Komplexen speisen. Dabei kann festgestellt werden, dass eine in all ihren Bestandteilen stringent und konsequent geformte Handlung ein Leitbild darstellt, das die Rezeption erleichtern soll. Aristoteles nimmt eine vorübergehende Komplexität in Kauf, wenn sie schließlich der Einheit förderlich ist, was zu einer qualitativen Aufwertung des Textes führt. Dennoch bleibt es stets Desiderat, den Text so zu gestalten, dass das Publikum nicht ausgeschlossen wird.

Fragen des Publikumsbezugs rücken bei Aristoteles vor allem in der Rhetorik in der

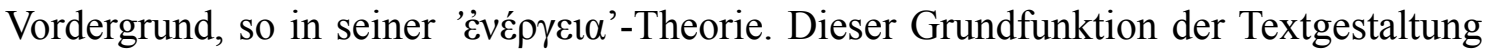
entspricht bei Quintilian das Prinzip 'clare atque evidenter ostendere' als Grundfunktionen der Textgestaltung, das sich als 'vor Augen führen', 'Augenscheinlichkeit' oder 'Anschaulichkeit' übersetzen lässt. ${ }^{77}$ Ziel ist es, ein möglichst detailliertes und wahrscheinliches Gesamtbild $\mathrm{zu}$ zeichnen, um dem Rezipienten eine möglichst verständliche Vorstellung des Vorgetragenen bzw. Geschriebenen zu bieten. Den Textproduzenten geben Aristoteles und Quintilian ein

\footnotetext{
$74 \mathrm{Vgl}$. Aristoteles 1994, 6, S. 21.

75 Ebd., 15, S. 49.

76 Vgl. ebd.. 15, S. 47.

77 Vgl. Aristoteles 2007, III, 10, S. 173-176 sowie Quintilian 2011, Bd. 2, VIII, 3 86, S. 187 auch ebd., IX, 2 40-42, S. 287.
} 
Inventar an Mitteln zur Hand, um dieses Prinzip umzusetzen und den Rezipienten im Wettstreit mit den Konkurrenten für sich zu gewinnen. Den Text für den Rezipienten verständlich zu gestalten, ist insgesamt eine inhärente Grundfunktion der Rhetorik.

Die Rhetorik befasst sich eingehend mit sprachlicher Gestaltung, setzt jedoch voraus, dass diese dem zu besprechenden Inhalt untergeordnet ist: 'Sachverhalte' dürften nicht eines 'hohle[n] Wortkult[s]' willen vernachlässigt werden. ${ }^{78}$ Quintilian knüpft daher dezidiert die Darstellungsmittel an den Inhalt. Unter diesem Grundsatz formuliert er die folgenden Stilprinzipien, die für Lesbarkeit von Bedeutung sind:

1. Die erste Qualität stellt die latinitas, der echte lateinische Ausdruck, dar. Das Prinzip umfasst zwei Komponenten: Erstens die Verwendung einer fehlerfreien Sprache und zweitens die Meidung von unrömischen (dies umfasst auch lokale Färbungen innerhalb des römischen Reiches außerhalb der Stadt Rom) bzw. ausländischen Ausdrücken. ${ }^{79}$

2. Die perspicuitas, die Durchsichtigkeit, steht für das Vermeiden von niedrigen Ausdrücken, die unter dem Rang des zu behandelnden Gegenstands oder der zu behandelnden Person liegen. Umgangssprache ist jedoch Quintilian zufolge vonnöten, wenn sie für einen behandelten Sachverhalt unentbehrlich ist. ${ }^{80}$ Aus dem Gebrauch gekommene Wörter, Ambiguitäten, Fehler, unverständliche Ausdrücke, falsche Verwendungen von Redefiguren und ein Wetteifern um unnötige Kürzen und Längen gelte es zu vermeiden; die wahre Leistung sei es, einen möglichst präzisen Ausdruck zu finden. ${ }^{81}$ Perspicuitas vermittelt ein Streben nach größtmöglicher sprachlicher Präzision, Deutlichkeit und Verständlichkeit.

3. Der Wortschmuck, ornatus, eröffnet dem Redner Spielraum, um seiner Rede und seinen Worten Erhabenheit, Großartigkeit, Glanz und Gewicht zu verleihen. Allerdings, schränkt Quintilian ein, 'läßt sich [niemals], was wirklich schön aussieht, trennen von der Zweckmäßigkeit'. ${ }^{82}$ Der Wortschmuck verleiht Quintilian zufolge -

\footnotetext{
78 Vgl. Quintilian 2011, Bd. 2, VIII, Vorrede 18, S.131.

79 Vgl. ebd.

80 Vgl. ebd., 2 2, S.139.

81 Vgl. ebd., 2 9, S.143.

82 Vgl. ebd., 3 11, S. 155.
} 
in Übereinstimmung mit den in der Einleitung der vorliegenden Arbeit behandelten Ausführungen des Aristoteles - dem Ausdruck einen Mehrwert, der ihn vor der Banalität bewahrt, jedoch muss auch der ornatus seine Funktion im Textgefüge erfüllen und darf nicht um seiner selbst willen zur Anwendung gebracht werden.

4. Das Prinzip der brevitas ist ein weiteres Mittel zur Präzision, Klarheit und Verständlichkeit. Quintilian wendet sich sowohl gegen ein 'Gewirr leerer Worte' als auch gegen ein 'Wetteifern um die Kürze'. ${ }^{83}$ Er strebt einen Mittelweg zwischen diesen beiden Extremen an, um einen Ausdruck zu finden, der so einfach und zugleich aussagekräftig als möglich ist.

5. Das aptum kann als austarierendes Element gelten, das in allen Bereichen zwischen qualitativem Anspruch und dem Wirkungszweck verhandelt. Quintilian schreibt in Bezug auf den Redeschmuck: 'Denn da [...] sich [der Redeschmuck] zu jeder Rede in anderer Form schickt, wird er, falls er den Gegenständen und Personen der Rede nicht angemessen ist, die Rede nicht nur nicht besser zur Geltung bringen, sondern sie sogar entwerten [...]. ${ }^{94}$ Das aptum speist sich aus dem Kontext und steht gegen die Annahme, dass nur ein stilistisch stark aufbereiteter Text qualitativ ansprechend ist. Wird eine angemessene Darstellung verfehlt, so zieht dies einen Qualitätsverlust nach sich, der sich insbesondere darauf bezieht, das entsprechende Publikum nicht erreichen zu können. ${ }^{85}$ Quintilian bezieht sich hier auf Cicero, der im Orator die Wahl der Stilhöhe ebenfalls vom Gegenstand des Textes abhängig macht. ${ }^{86}$ Bei Quintilian avanciert das aptum anders als bei Cicero zum 'allernotwendigste[n]' Vorzug des Ausdrucks. ${ }^{87}$ Das aptum ist demnach ein zentrales Prinzip einer

\footnotetext{
83 Vgl. ebd., 2 17-19, S. 147.

84 Ebd., XI, 12 , S. 545.

$85 \mathrm{Vgl}$. ebd.

86 Vgl. Cicero 2004, insbesondere Kapitel 76-101.

87 Quintilian 2011, Bd. 2, XI, 1 2, S. 545. Eine fundierte Darstellung der Rhetorik und Einführung in die Beziehungen zwischen ihren Theoretikern liefert Ottmers 2007.
} 
Wirkungsästhetik, das die Anforderungen des Publikums mit den Ansprüchen des Autors in Einklang bringt. ${ }^{88}$

Quintilians Ausführungen verdeutlichen, dass in den Texten der antiken Rhetorik nicht von einer einheitlichen sprachlichen Gestaltung gesprochen werden kann. Vielmehr handelt es sich bei der sprachlichen Gestaltung sowie auch der Wirkung um einen dynamischen Prozess, der im Spannungsfeld zwischen Inhalt, Stilistik und dem erwünschten Zweck abläuft. Dementsprechend ist auch zur Analyse der Lesbarkeit eine genaue Betrachtung des Zusammenspiels notwendig, das sich selbst innerhalb eines Werkes immer neu rekonfigurieren kann. Zudem zeigt die Analyse von Quintilians Auffassung des aptum, dass dabei der Frage nach der Qualität eine eminente Bedeutung zukommt.

In Bezug auf den Wert eines Werkes und auf die Legitimation von Lesbarkeit kommt der Wirkungsfunktion eines Textes eine bedeutende Rolle zu. Für die klassische Rhetorik war insbesondere Ciceros Unterteilung in die Funktionen des docere/probare, delectare/conciliare und moverelflectere wirksam. ${ }^{89}$ So heißt es etwa in De oratore:

So konzentriert sich die gesamte Redekunst auf drei Faktoren, die der Überzeugung dienen: den Beweis der Wahrheit dessen, was wir vertreten, den Gewinn der Sympathie unseres Publikums und die Beeinflussung seiner Gefühle im Sinne dessen, was der Redegegenstand jeweils erfordert. ${ }^{90}$

Hier zeigt sich zunächst, dass das aptum auch für die Wirkungsfunktionen relevant ist, indem jene dem Sujet der Rede entsprechend eingesetzt werden müssen. Cicero strebt keine hierarchische Unterscheidung zwischen den officia oratoris an, sondern etabliert sie als gleichwertig. Deutlich wird der Publikumsbezug, der allen Überlegungen hinsichtlich der Wirkungsabsichten zugrunde liegt. Bereits Horaz adaptiert Ciceros Wirkungsfunktionen für die Dichtkunst und verfolgt dabei explizit verschiedene Arten des Erfolgs:

\footnotetext{
88 Erich Auerbach führt in Mimesis. Dargestellte Wirklichkeit in der abendländischen Literatur (1946), seiner Studie zur Mimesistradition, das aptum als Legitimationsstrategie einer realistischen Literatur an, die er in Bezug auf die Rhetorik höhenmetaphorisch einordnet: 'Indem Stendhal und Balzac beliebige Personen des täglichen Lebens in ihrer Bedingtheit von den zeitgeschichtlichen Umständen zu Gegenständen ernster [...] Darstellung machten, zerbrachen sie die klassische Regel von der Unterscheidung der Höhenlagen, nach welcher das alltägliche und praktisch Wirkliche nur im Rahmen einer niederen oder mittleren Stilart, das heißt [...] als [...] leichte [...] und elegante Unterhaltung seinen Platz in der Literatur haben dürfte.' (Auerbach 1977, S. 515).

$89 \mathrm{Zu}$ den officia oratoris in der rhetorischen Tradition vgl. Ottmers 2007, v.a. S. 127-131 und 205-210.

90 Cicero 2001, 2 (115), S. 279. Siehe ebenso Cicero 2004, 21 (69), S. 65.
} 
Entweder nützen oder erfreuen wollen die Dichter oder zugleich, was erfreut und was nützlich fürs Leben ist, sagen. [...] [Bei öffentlichen Abstimmungen erhielt] jede Stimme [...], wer Süßes und Nützliches mischte, indem er den Leser ergötzte und gleicherweise belehrte. Solch ein Buch verdient [...] die Groschen, gelangt übers Meer und verlängert seinem bekannten Verfasser die Lebensdauer. ${ }^{91}$

Horaz bevorzugt das bekannte Diktum des docere et delectare. Es findet sich die Metapher des Süßens, die für eine angenehme Aufbereitung von bedeutenden Sachverhalten steht und in der Lesbarkeitsdebatte Tradition hat. Die Kombination der beiden Wirkungsfunktionen dient Horaz sowohl als Mittel zum finanziellen Erfolg, der zudem nicht auf das Kernland des Römischen Reiches beschränkt ist, sondern in die Provinzen reicht, als auch zum Erlangen von Nachruhm, der in der deutschen Tradition vor allem durch Immanuel Kant Prominenz erlangt und eine prestigeträchtige Form des Erfolgs darstellt. Im Hinblick auf die Analyse der Autorpoetiken in den nachfolgenden Kapiteln gilt es demnach $\mathrm{zu}$ beachten, in welchen Kontexten Autoren die Wirkungsabsichten zu welchem Zweck einsetzen, und, ob Autoren eine Wirkungsabsicht oder eine Kombination von zweien gegenüber den anderen privilegieren und warum. Zudem gilt es zu analysieren, wie Autoren das Verlangen nach Lesbarkeit $\mathrm{zu}$ den Wirkungsfunktionen in Bezug setzen und welche Arten der Kommunikation mit dem Publikum daraus resultieren.

Die Relevanz der Narratologie sowie der poetologischen und rhetorischen Tradition für Lesbarkeit ergibt sich daraus, dass allen eine Dynamik eingeschrieben ist, die verschiedene Zugangsmöglichkeiten erlaubt. Zwischen den stabilen Parametern kommt es zu einem produktiven Zusammenspiel, das vielfältige Konstellationen zulässt, wodurch Lesbarkeit die Flexibilität erhält, sich verschiedenen Kontexten und Anforderungen anzupassen. Dabei bewegt sie sich zwischen den Extrempunkten des Einfachen und des Komplexen, die kompensatorisch aufeinander wirken können, um den Bezug zum Publikum zu gewährleisten. Franzens poetologisches Werk fungiert komplementär zur wissenschaftlichen Narratologie als exemplarische Autorpoetik, die sich intensiv mit Lesbarkeit und ihrem Wirkungspotenzial auf die Leserschaft auseinandersetzt. Es soll aufgezeigt werden, dass sich Franzens Theorie einer von 
Lesbarkeit inspirierten Erzählliteratur des frühen 21. Jahrhunderts intensiv mit theoretischen Aspekten der klassischen Poetik und Rhetorik auseinandersetzt. Dadurch kann aufzeigt werden, in welcher Form diese im Lesbarkeitsdiskurs nach der Jahrtausendwende präsent sind. Zunächst verurteilt Franzen in seinem Essay 'Mr. Difficult' Autoren, die schwierige Bücher schreiben, und Kritiker, die sie weiterempfehlen: 'a difficult novel just sits there on your shelf unread - unless you happen to be a student, in which case you're obliged to turn the pages of Woolf and Beckett.' ${ }^{92}$ Es spiegelt sich hier Fiedlers Verlangen nach einer Umerziehung der Kritik. ${ }^{93}$ Franzen macht geltend, dass schwer lesbare Bücher selten gelesen werden, und etabliert Lesbarkeit als Grundvoraussetzung für Breitenwirkung. Gleichzeitig lanciert er eine versteckte Kritik am akademischen Betrieb, dessen Kanon schwer zugängliche Werke begünstigt und fördert. An der Kritik bemängelt Franzen, dass ein komplexer Roman wie A Frolic of His Own (1994) des amerikanischen Autors William Gadiss von der Kritik Elogen erhält, die zur zweiten Auszeichnung des Autors mit dem National Book Award geführt hätten:

it's riddled with motifs, quotations, stories within stories, and countless allusions to Gaddis's own earlier works and other famous texts [...], and its only aesthetic weakness, really, is that much of it is repetitive, incoherent, and insanely boring. ${ }^{94}$

Franzen kritisiert eine Verkomplizierung des Werkes, wobei sich Anklänge an den in der Einleitung der vorliegenden Arbeit eingeführten aristotelischen Begriff des 'Rätsels' finden. Während Aristoteles jenen auf eine komplexe Stilistik anwendet, die zur Unverständlichkeit eines Textes führt, adressiert Franzen strukturelle und thematische Aspekte. Die ästhetischen Schwächen des Romans macht Franzen an Aspekten fest, die als Nichtbeachtung gewisser Prinzipien der klassischen Rhetorik und Poetik interpretiert werden können: Unnötige Wiederholungen widersprechen dem Prinzip der brevitas, eine inkohärente Handlung läuft der von Aristoteles in seiner Poetik geforderten stringenten und schlüssigen Handlungsführung zuwider und Langeweile erfüllt nicht die Wirkungsabsicht des delectare.

Die Komplexität und der Publikumsbezug stellen in Franzens Essay die zentralen Fragestellungen dar. Als Ausgangspunkt wählt Franzen den Vorwurf einer Leserin, sein

\footnotetext{
92 Franzen J. 2002.

$93 \mathrm{Vgl}$. die Seiten 7 und 8 der vorliegenden Arbeit.

${ }^{94}$ Franzen J. 2002.
} 
Roman The Corrections sei zu schwierig, um einer breiten Leserschaft leicht zugänglich $\mathrm{zu}$ sein und der komplexe Stil sei auf eine intellektuelle New Yorker Elite zugeschnitten. ${ }^{95}$ Dementsprechend fragt die Leserin den Autor: 'Who is it that you are writing for? It surely could not be the average person who just enjoys a good read.' 96 Die Leserin stellt somit sprachliche Komplexität in Opposition zu Vergnügen und einem 'good read'. Diese Unterscheidung geht mit der Einteilung in eine vermeintliche intellektuelle Eliteleserschaft und einen durchschnittlichen Leser, der unterhalten werden will, einher. Sie kritisiert die explizite Zurschaustellung einer intellektuellen Überlegenheit, die sie an der Komplexität des Romans festmacht. Franzen bedauert, dass die Dame sich durch seine intellektuelle Textgestaltung ausgeschlossen fühlte ('felt excluded'). ${ }^{97}$ Seine Metaphorik deutet auf eine Konfiguration der Leserschaft hin, die umfassend ist und den Ausschluss von Lesern aufgrund einer zu großen Komplexität meidet, um ein möglichst großes und diverses Publikum zu erreichen.

Franzen nutzt die Reflexion über den Publikumsbezug zum Entwurf zweier Modelle. Zunächst entwirft er ein auf das Prestige des Autors und des Lesers bezogenes 'Status model':

From a Status perspective, difficulty tends to signal excellence; it suggests that the novel's author has disdained cheap compromise and stayed true to an artistic vision. Easy fiction has little value, the argument goes. Pleasure that demands hard work, the slow penetration of mystery, the outlasting of lesser readers, is the pleasure most worth having; and if, like Mrs. M---- [die Leserin, die sich über die Schwierigkeit von Franzens Roman beklagt hatte], you can't hack it, then to hell with you. ${ }^{98}$

Die Grundprämisse dieses Modells ist eine relative Indifferenz des Autors gegenüber den Bedürfnissen der Leserschaft. Der künstlerische Anspruch wird durch das religiöse

\footnotetext{
95 Auch Franzens Tübinger Vorlesung zeigt seine Präferenz für Lesbarkeit. Er schreibt, dass er eine Literatur ablehne, die 'offenes Didaktisieren [und] unnötige Schwierigkeiten' anstrebe (S. 11). Durch eine Traummetapher weist Franzen dem Leser die zentrale Position in der Literaturproduktion zu: 'Was ist denn Fiktion, wenn nicht eine Form des zielgerichteten Träumens? Ein Autor arbeitet ja daran, einen Traum zu schaffen, der lebendig und so bedeutsam ist, dass der Leser ihn lebhaft träumen [...] kann' (S. 17). Dies gleicht dem rhetorischen Begriff des 'Anschaulichmachens'. Um die Ausrichtung auf den Leser umzusetzen, müsse ein Autor darauf achten, 'was funktionieren kann, was er plausibel machen kann, was er lesbar machen kann, was er verständlich machen kann, was er unterhaltsam machen kann' (S. 14). Lesbarkeit ist für Franzen ein Überbegriff, der darauf verweist, dass etwas überhaupt gelesen werden kann, während Verständlichkeit als leichtes Textverständnis gelten kann. Plausibilität kann für eine innere Folgerichtigkeit stehen, was der Poetik des Aristoteles entstammt. Franzen unterscheidet dann zwischen 'Unterhaltungsschriftstellern' und 'literarischen Werken' (S. 18). Er macht einen Qualitätsanspruch geltend, der ihn von bloßer Unterhaltung absetzt, sieht aber Unterhaltung als inhärente Qualität von dezidiert ‘literarischen Werken’; Franzen J./Haslett 2010.

96 Franzen J. 2002.

97 Ebd.

98 Ebd.
} 
Bild der Vision in einen prophetischen Kontext gesetzt, wodurch die Position des Autors gegenüber der des Lesers überhöht und der Trennung zwischen den Positionen Nachdruck verliehen wird. Konkreten Ausdruck findet dies im Schwierigkeitsgrad eines Textes. Komplexität wird privilegiert, wohingegen ein einfach zu lesender Text und eine Position der Mitte abgelehnt werden. Diese Vorstellung basiert auf einem Vergnügensbegriff, der auf einen intellektuellen Leser zielt und ein breites Publikum ausschließt. Das Vergnügen für den Leser besteht nicht darin, eine möglichst leichte und unterhaltsame Lektüre zu genießen, sondern in der Überwindung von Schwierigkeiten. Wem diese gelingt, wird zum würdigen, elitären Leser, der auf die breite Masse herabsieht. Der Wert eines Werkes liegt demnach ausschließlich in der Ästhetik und definiert sich nicht über den Erfolg bei der Leserschaft.

Dem stellt Franzen das 'Contract model' gegenüber, in dem es die Pflicht des Autors ist, dem Vertragspartner, dem Leser eine 'pleasurable experience' zu bereiten. ${ }^{99}$ Der Fokus der Publikumsunterhaltung stärkt die Position des Lesers und führt zu einem Streben nach der Mitte: 'Writing thus entails a balancing of self-expression and communication within a group, whether the group consists of "Finnegans Wake" enthusiasts or fans of Barbara Cartland. ${ }^{100}$ Es geht Franzen sowohl um künstlerischen Anspruch als auch um die Zufriedenstellung einer breiten Leserschaft. Die entscheidende Kategorie ist die Kommunikation, die den Autor in die Gemeinschaft mit dem Leser einbettet und letzterem Agens verleiht. Die Leserschaft konzipiert Franzen möglichst divers, indem er die Extrempunkte von Joyces komplexem Klassiker der Moderne und den populären Liebesromanen der britischen Bestsellerautorin setzt, deren beider Fans er mit seinen Werken zufriedenstellen will. Aus seinen Überlegungen folgert er in Bezug auf das Autor-Leser-Verhältnis:

Every writer is first a member of a community of readers, and the deepest purpose of reading and writing fiction is to sustain a sense of connectedness, to resist existential loneliness; and so a novel deserves a reader's attention only as long as the author sustains the reader's trust. [...] The discourse here is one of pleasure and connection. ${ }^{101}$

\footnotetext{
99 Ebd. Dass die Vertragsmetapher in der englischsprachigen Literatur Tradition hat, zeigt Fionnuala Dillanes Analyse der Rezeption von George Eliots Impressions of Theophrastus Such (1879). Besonders relevant ist die Rezension von Grant Allen, ein damaliger Kritiker für Fortnightly Review, welcher der Autorin vorgeworfen hatte, keine Handlung konstruiert zu haben, was er als 'breach of contract with the public' einordnet. Vgl. Dillane 2013, S. 181f und Allan 1897, S. 149.

100 Franzen 2002.

101 Ebd.
} 
Die Position des Autors gegenüber dem Leser ist im Gegensatz zum 'Status model' weder exponiert noch hermetisch abgeschlossen, sondern egalitär. Die Vertragsmetapher kreiert ein schriftstellerisches Selbstverständnis, in dem die Autoren dazu verpflichtet sind, während des Schreibprozesses nicht nur den eigenen, sondern nachdrücklich auch den literarischen Ansprüchen des Lesers gerecht zu werden. Um als Schriftsteller den Vertrag einzuhalten, fungiert das Vergnügen als entscheidende Komponente zur Zufriedenstellung des Publikums, was für ein auf Lesbarkeit und Unterhaltung zielendes Literaturprogramm spricht. ${ }^{102}$

Franzen diskutiert zunächst das jeweilige Modell aus Sicht der Opposition und unterstreicht dadurch den inhärenten Wettkampfcharakter des Lesbarkeitsdiskurses. Die Anhänger des 'Status model' erschienen demzufolge der Contract-Fraktion als 'arrogant connoisseurial elite', während die Anhänger des 'Status model' die Einhaltung des Vertrags als 'recipe for [...] aesthetic compromise' sehen würden. ${ }^{103}$ Die Kritik am 'Contract model' gleicht Halversons Infragestellung der 'Neuen Deutschen Lesbarkeit', da der Wunsch, für eine möglichst breitgefächerte Leserschaft zu schreiben, als Anbiederung gesehen wird, die notwendigerweise einen Qualitätsverlust nach sich zieht. ${ }^{104}$ Die Privilegierung von Komplexität gegenüber der Publikumswirksamkeit führt Franzen zufolge zum Ausschluss großer Leserkreise. Die Kritik am 'Status model' wendet sich gegen diese Exklusivität, durch die eine intellektuelle Elite ihren Status zu wahren versucht. Mit dieser integrativen Auffassung nimmt Franzen die Autoren in die Pflicht, ihre Werke für eine breite Leserschaft zugänglich zu gestalten.

Im Anschluss an die Gegenüberstellung der beiden Modelle bezieht Franzen Position:

The Status position is undeniably flattering to the writer's sense of importance. In my bones, though, I'm a Contract kind of person. I grew up in a friendly, egalitarian suburb reading books for pleasure and ignoring any writer who didn't take my entertainment seriously enough. Even as an adult, I consider myself a slattern of a reader. ${ }^{105}$

\footnotetext{
102 Die Schwierigkeit des Textes kann als entscheidendes Distinktionsmerkmal gelten. Während diese im 'Status model' als Qualitätsmerkmal angesehen wird, ergebe sie im Hinblick auf das 'Contract model' Probleme: 'difficulty is a sign of trouble. In the most grievous cases, it may convict an author of placing his selfish artistic imperative or his personal vanity ahead of the audience's legitimate desire to be entertained' (Ebd.).

103 Ebd.

104 Vgl. Seite 14 der vorliegenden Arbeit.

105 Franzen J. 2002.
} 
Franzen satirisiert die Status-Fraktion, indem er dieses Modell als Auswuchs einer narzisstischen schriftstellerischen Selbstwertschätzung darstellt. Er schlägt sich dezidiert auf die Seite der Contract-Fraktion, wobei diese Entscheidung von einer Identifikation mit dem Unterhaltungsbedürfnis der Leserschaft geprägt ist. Dadurch etabliert Franzen Unterhaltung als vollwertige Qualitätseigenschaft. In Bezug auf die Lesbarkeitstradition erweist sich die erotische Metapher am Ende des Zitats als interessant. Der österreichische Schriftstellers Joseph August Lux etwa attestiert in der expressionistischen Zeitschrift Der Sturm (1910) Autoren, die leicht verständliche Texte schreiben, um eine große Leserschaft für sich $\mathrm{zu}$ gewinnen, die 'schamlose Verlogenheit' einer 'Dirne'. ${ }^{106}$ Im Gegensatz zu Franzen legt Lux den Fokus auf die Autoren und ihre finanziellen Interessen, die er stigmatisiert. Derselbe Prozess liegt Wittstocks 'Prinzip der Verführung' und Greiners Leseverführer zugrunde, bei denen die Bemühungen von Autoren um ein großes Publikum jedoch positiv besetzt sind. ${ }^{107}$ Franzen bewegt sich im gleichen semantischen Feld und rekurriert auf das Bild einer sexuell libertinären Person mit fragwürdigem Charakter, das er auf die eigenen Lesebedürfnisse anwendet. Ihm geht es um Leser, die aktiv danach streben, ihr Bedürfnis nach Unterhaltung zu befriedigen. Zwar verwendet Franzen mit 'slattern' einen Terminus, der negativ behaftet ist, unterläuft diese Konnotation jedoch mit seinem Bekenntnis zur Unterhaltung. Dadurch legitimiert er den Lustgewinn für das Publikum durch Unterhaltung und etabliert diese als Kategorie, die für Autoren ein selbstverständlicher Bestandteil ihrer ästhetischen Überlegungen sein sollte.

Nach seinem Plädoyer für Unterhaltung eruiert Franzen den Begriff der 'difficulty' am Beispiel von William Gaddis' Roman The Recognitions (1959). Als enthusiastischen Verfechter des 'Status model' nennt Franzen Gaddis 'quintessentially difficult'. ${ }^{108}$ Demnach unternimmt er mit diesem Paradebeispiel eine poetologische Definition von 'difficulty'. Zugleich erinnert der Titel von Franzens The Corrections an ebenjenen Roman. Franzen arbeitet sich an der 'difficulty' der Recognitions ab, um aufzuzeigen, warum er selbst für seine Romane einen am 'Contract model' orientierten Weg eingeschlagen hat. In diesem Sinne kann Franzens Essay auch als Apologie für Lesbarkeit gelten.

\footnotetext{
106 Lux 1910, S. 14.

107 Vgl. Wittstock 1995, S. 15 und Greiner 2007.

108 Ebd.
} 
Die Komplexität der Recognitions führt Franzen übergreifend auf Gaddis' 'suspicion of realism' zurück. ${ }^{109}$ Eine realistische Gestaltungsweise kann demnach als Indiz für Lesbarkeit gelesen werden. Dies drückt sich auch in der Verwendung des Vokabulars 'being unreadable, sometimes even unintelligible' aus. ${ }^{110}$ 'Unreadable' fungiert im Vergleich zu 'unintelligible' als weniger intensive Form der 'difficulty'. Während 'unreadable' eine Textform suggeriert, die schwierig zu lesen ist, steht 'unintelligible' für eine Textform, die keinerlei Erschließung des Textes mehr zulässt. Konkret etabliert Franzen folgende Parameter, die eine hohe Komplexität bedingen:

obscure references [...], precipitous discourses [...]. The novel came in page-long paragraphs in which oxygen was at a premium, and the emotional temperature of the novel started cold and got colder. The hero [...] was likeable [...] but otherwise the author's satiric judgements and intellectual obsessions discouraged intimacy. It was a struggle to figure out what, or even who, the story was about; dialogue was punctuated with dashes and largely unattributed; [...] I was alone and unprepared on a steep-sided, frigid, airless, poorly mapped mountain. ${ }^{111}$

Franzen ermittelt hier die Elemente, die zu einer Verkomplizierung von Texten führen. Markant ist zunächst die Bergmetapher, die er dazu heranzieht, um die Pflicht eines Autors zu demonstrieren, die Leser ohne größere Mühen auf den Berg zu führen, welche Gadiss dem Leser Franzen zufolge schuldig bleibt. Gaddis bringt komplexes Bildungsgut ein, das sich unter literarische Referenzen und intellektuelle Diskurse subsumieren lässt, was eine thematische Komplexität anzeigt. In Franzens Ausführungen zur Textgestalt finden sich wiederum mehrere Aspekte, die sich zur klassischen Rhetorik und Poetik in Bezug setzen lassen: Die enorme Länge der Absätze widerspricht vom Effekt her dem rhetorischen Prinzip der brevitas. Zudem betont Franzen die Unzugänglich der Handlung von Gaddis's Roman, was er an Kategorien festmacht, die als Mangel an claritas interpretiert werden können, der sich in der Ambiguität des Status vom Gesagten ausdrückt. Außerdem nennt Franzen ein mangelndes Identifikationsangebot mit der Hauptfigur und ein mangelndes Ansprechen der Gefühle als Hindernisse für die Verständigung zwischen Autor und Leser, was ebenfalls den Grundsätzen der klassischen Rhetorik widerspricht.

\footnotetext{
109 Vgl. ebd.

110 Ebd.

111 Ebd.
} 
Franzen kritisiert vor allem das Fehlen von Elementen, die dem Handlungsverlauf eine klar nachzuvollziehende Struktur verleihen, und er etabliert in diesem Kontext Mittel, die Lesbarkeit bedingen:

' $\mathrm{J}$ R' is a seven-hundred-and-twenty-six-page novel consisting almost entirely of overheard voices, with nary a quotation mark, no conventional narration of any kind, no 'later that same evening,' no 'meanwhile in New York,' not a single chapter break, not even a section break, but thousands of dashes and ellipses, another cast of dozens, and a laughably complicated plot [...].112

Franzen führt folgende Aspekte als lektüreerschwerend ein, die als Nichtbeachtung der rhetorischen Prinzipien der claritas und der perspicuitas gesehen werden können: einen Mangel an strukturierenden Satzzeichen, ordnenden Abschnitten, Hinweisen auf die Chronologie des Handlungsverlaufs sowie dessen Verortung in Schauplätze, eine Überbesetzung im Figureninventar und eine übermäßig komplexe Handlungsführung. Insgesamt kritisiert Franzen eine Abkehr vom Ziel des traditionellen Erzählens, eine folgerichtige und für die Leser verständliche Geschichte zu schreiben. Dies wird für Franzen zum zentralen Aspekt des Schreibens: 'Fiction is storytelling'. ${ }^{113}$ Durch die Gleichsetzung von fiktionalem Schreiben und Erzählen avanciert das Erzählen im Literaturverständnis Franzens zur elementaren Kategorie des 'Contract model' und damit zum Lesbarkeit bedingenden Parameter.

In den vorausgehenden Ausführungen wurde Lesbarkeit zu Aspekten der Narratologie des frühen 21. Jahrhunderts sowie zur aristotelischen Mimesistradition und der klassischen Rhetorik in Bezug gesetzt, die sich mit der Wirkung auf das Publikum befassen. So konnten verschiedene Kategorien als Bezugspunkte erschlossen werden, deren Bedeutung für den Lesbarkeitsdiskurs im frühen 21. Jahrhundert am Beispiel von Franzens Autorpoetik analysiert wurde. Die Analyse von Lesbarkeit in den folgenden Kapiteln wird mit diesen Kategorien arbeiten. Dabei wird vorausgesetzt, dass der Lesbarkeit eine Dynamik eingeschrieben ist, die sich jeweils aus dem zeitgeschichtlichen Hintergrund und aus der Auseinandersetzung mit früheren Erscheinungsformen sowie auch mit anderen literarischen Ästhetiken ergibt. Lesbarkeit wird als flexibles, wandlungsfähiges Prinzip verstanden, bei dem es immer auf die 
Vielfalt und Bedeutung der Konstellationen und den Zweck ihrer Anwendung ankommt. Durch dieses ihr inhärente komplexe Zusammenspiel führt eine Untersuchung von Lesbarkeit in ein umfassendes erzählerisches Terrain. Sie speist sich aus den Extrempunkten des Einfachen und der Komplexität, die dynamisch und teils kompensatorisch aufeinander einwirkend die Tendenz zu einem Mittelweg eröffnen. Dieser Mittelweg ist an die Wirkungsfunktion der Unterhaltung gekoppelt und soll eine breite Leserschaft ansprechen. Zugleich macht die Dynamik und Wandelbarkeit des Zusammenspiels der poetologischen Parameter eine intensive Untersuchung konkreter literarischer Werke notwendig, an denen die Wirkung von Lesbarkeit erst verstanden werden kann. 


\section{Kapitel 2: Lesbarkeit in der Tradition deutscher Erzählliteratur}

Das Kapitel untersucht diachronisch die Konstellationen des Lesbarkeitsbegriffs in der neueren deutschen Literaturgeschichte. Als Ausgangspunkt dient das frühe 18 . Jahrhundert, da in Deutschland um diese Zeit die Grundvoraussetzungen für jenen Begriff von Lesbarkeit geschaffen wurden, der im Literaturstreit im Vordergrund stand: Im Bürgertum verbreitete sich zusehends die Lesefähigkeit, was in Kombination mit der Entwicklung eines Literaturmarktes $\mathrm{zu}$ tiefgreifenden Veränderungen in der Literaturlandschaft führte. War die Leserschaft bis dahin auf einen limitierten Kreis beschränkt, konnte nun zunehmend ein breiteres Publikum mit deutschsprachiger Literatur versorgt werden. Dabei gilt es jedoch zu beachten, dass Deutschland zum damaligen Zeitpunkt aus zahlreichen Kleinstaaten bestand und etwa im Vergleich zu den Nationalstaaten Großbritannien oder Frankreich nicht von einer umfassenden Leserschaft gesprochen werden kann. Die einsetzende Loslösung der Literaturproduktion und -rezeption von Hof, Kirche und Universitäten machte Autoren allmählich von diesen mäzenatischen Institutionen weniger abhängig. Die Ausbildung des literarischen Marktes ermöglichte es den Autoren grundsätzlich, auch wenn dies noch keineswegs üblich war, mit der Schriftstellerei zumindest einen Teil ihres Lebensunterhalts $\mathrm{zu}$ verdienen, wobei das Verlangen nach Verkaufserfolg heftig umstritten war. ${ }^{114}$

In diesem Prozess avancierte die Prosa zu einer besonders umstrittenen Gattung, denn die geistlichen und weltlichen Eliten sahen diese in der antiken Poetik nicht etablierte Form als poetologisch minderwertig an. Dies führten sie vor allem auf deren Unterhaltungsfunktion zurück, während dem Lesen bis dahin hauptsächlich eine Bildungsfunktion zugekommen war. ${ }^{115}$ Besonders manifestiert sich dies in der Gattung des Romans, die in den ersten beiden Jahrzehnten des 18. Jahrhunderts in intellektuellen Kreisen nicht akzeptiert war. Deutlich wird dies anhand der Berufsbezeichnungen: diejenigen, die im Bereich der traditionell 'hohen' Gattungen Epos, Trauerspiel und Ode wirkten, wurden vorzugsweise als 'Dichter' bezeichnet; wer in der 'niederen' Form der

\footnotetext{
114 Hans Jürgen Haferkorn terminiert in seiner 'berufsständischen Biographie' das frühe 18. Jahrhundert als Beginn dieser Entwicklung. Die endgültige Ausprägung des freien Schriftstellertums siedelt Haferkorn dann in der zweiten Hälfte des 18. Jahrhunderts an; vgl. Haferkorn 1963, S. 183.

115 Vgl. Aust 2000, Kleinschmidt 2003 a, Kleinschmidt 2003 b sowie Kohl 2007 b, insbesondere S. 416-426.
} 
Komödie oder des Romans schreibt, galt als 'Schriftsteller'. ${ }^{116}$ Albert Ward sieht in Book Production, Fiction and the German Reading Public 1740-1800 (1974) erst mit der deutschen Erstveröffentlichung von Daniel Defoes The Life and Strange Adventures of Robinson Crusoe of York (1719) einen Wendepunkt gegeben, da es Defoe gelungen sei, Unterhaltung durch die Verbindung mit der prestigeträchtigeren didaktischen Funktion zu legitimieren. ${ }^{117}$

Die diachronische Untersuchung soll traditionelle Ausprägungen des Lesbarkeitsbegriffs sowie punktuell die ihnen zugrundeliegenden historisch spezifischen gesellschaftlichen, ökonomischen und sozialen Umstände analysieren. Das Kapitel wird poetologische Äußerungen ausgewählter Vertreter des Literaturbetriebs erschließen sowie deren nicht-literarischen Motivationen und theoretischen Parameter identifizieren. Dabei wird chronologisch ein Bogen von 1700 bis zur deutschen Nachkriegsliteratur vor 1990 geschlagen, der die Vielfalt der Konstellationen und die Dynamik des Lesbarkeitbegriffs hervorheben wird. Anhand der untersuchten Beispiele soll aufgezeigt werden, wie Autoren und andere am Literaturbetrieb Beteiligte Lesbarkeit verstehen und für die Legitimation ihrer Literaturvorstellung einsetzen.

Einer der ersten Autoren, der sich die neuen Marktbedingungen zu Nutze machte und mit seinem frühen Prosawerk zu einem der meistverkauften deutschsprachigen Autoren um 1700 wurde, war der unter dem Pseudonym Menantes veröffentlichende Christian Friedrich Hunold. ${ }^{118}$ Hunolds Romane unterscheiden sich von vielen Werken seiner Zeit, die zumeist als Nebenbeschäftigung höfischer oder geistlicher Beamter und Würdenträger geschaffen wurden, da sie hauptsächlich dazu konzipiert waren, ihrem Autor die Existenz zu sichern. ${ }^{119}$ Zwar übte Hunold selbst kleinere Ämter aus, er musste sich jedoch, wie Hans Wagener hervorhebt, auf seine literarischen Einkünfte verlassen:

Das Schreiben von Büchern war für ihn zu dieser Zeit also keine Beschäftigung müßiger Nebenstunden, nicht das intellektuelle Spiel eines kultivierten Adeligen

\footnotetext{
116 Vgl. Kiesel/Münch 1977, S. 94f.

117 Vgl. Ward 1974, S. 18-25.

118 Laut Hans Wageners Ausführungen im Vorwort zu Die Verliebte und Galante Welt erreichte Hunold die zweithöchste Auflagenzahl seiner Zeit; vgl. Wagener 1988, S. 9.

119 Der Dichter und Verleger Benjamin Neukirch schreibt in der Vorrede zu seiner Anthologie Herrn von Hoffmannswaldau und andrer Deutschen auserlesener und bißher ungedruckter Gedicht (1697): '[Die Dichter] müssen über dieses entweder selbst mittel / oder doch auskömmlichen unterhalt / und zum wenigsten bei ihren amts-geschäften die freyheit haben / daß sie drey oder vier stunden des tages verschwenden dürffen' (Neukirch 1961, S. 18).
} 
oder hohen bürgerlichen Beamten, sondern in erster Linie Mittel zum Erwerb des Lebensunterhalts. ${ }^{120}$

Die zeitgenössischen Gepflogenheiten hielten Hunold demnach nicht davon ab, finanziellen Erfolg anzustreben. ${ }^{121}$ Auf den Beginn des 18. Jahrhunderts zurückblickend schreibt Goethe in Dichtung und Wahrheit (hier 1825) über das Verhältnis zwischen Autor, Literatur und Verdienstmöglichkeiten, dass die Produktion von poetischen Schriften als etwas 'Heiliges' angesehen wurde und man es beinahe für 'Simonie' hielt, ein Honorar zu nehmen. ${ }^{122}$ Goethe rekurriert dabei sowohl auf die christliche Tradition als auch auf die antike Tradition des poeta vates. Den Dichter versieht er mit einer göttlichen Funktion, die es ihm versagt, eine Entlohnung anzunehmen, wodurch er ein antipodisches Verhältnis zwischen künstlerischem Ansehen und finanziellem Erfolg kreiert, der als anrüchig gilt.

Um Geld zu verdienen, strebt Hunold in der Vorrede zum zweiten Teil des Romans Die Verliebte und Galante Welt (1700) folgende Wirkung an: allen Lesern soll 'ein vergnügt Gemühte' bereitet werden. ${ }^{123}$ Das Vergnügen deutet auf die rhetorische Wirkungsabsicht des delectare hin, das Gemüt auf das movere. Neben der Mischung von Vergnügen und Gefühlsregung führt Hunold in der Vorrede zum ersten Teil desselben Romans mit dem Erzählen einer 'wahrhaftige[n] Geschichte' eine weitere Strategie zur Erreichung von Breitenwirkung an. ${ }^{124}$ Gemeint ist eine plausible realistische Darstellung tatsächlich geschehener Begebenheiten. So stehen etwa in Der Europäischen Höfe Liebes- und Heldengeschichte (1705) Skandale von Mitgliedern europäischer Herrscherhäuser im Fokus. Als Ziel benennt Hunold in den Academischen Nebenstunden (1713), dem Leser durch das Wiedererkennen und Erraten gewisser Personen oder Ereignisse Vergnügen bereiten zu wollen.

Hunolds Einstellung zu seinen Romanen wandelte sich drastisch, als er eine akademische Karriere einschlug. Da er der Ansicht war, dass unterhaltende Prosa nicht mit seriöser akademischer Arbeit zu vereinbaren sei, distanzierte er sich in seinen Academischen Nebenstunden dezidiert von seinem Frühwerk:

\footnotetext{
120 Wagener 1988, S. 9.

121 Der Autor Christian Weise macht in seiner poetologischen Schrift Curiöse Gedanken Von Deutschen Versen (1692) geltend, dass nur dann 'die Poeterey aestimirt [wird], wenn der Mann etwas anders darneben hat, davon er sich bey Mitteln und bey respect erhalten kan' (Auszug in Szyrocki 1977, S. 234).

122 Vgl. Goethe 1985-1999, Abteilung 1, Bd. 14, S. 563.

123 Hunold 1988 b, S. 5.

124 Hunold 1988 a, S. 4 bzw. 5.
} 
Tugendhaffter Leser / ich wünschte / daß viele meiner Schrifften in ihrer ersten Gebuhrt erstickt wären. [...] Ich war jung; von Tugenden besaß ich nichts / und von Wissenschafften hatte ich wenige Kenntniß / und gleichwohl wollte ich hoch hinaus. Ich hatte von der Adler ihrem Flug zur Sonnen gehöret; und gedachte mit den blöden Augen meines verfinsterten Verstandes eine so jähe Bahn gleichfals zufinden. Allein ich geriehte mit den Sinnen unter die Eulen / welche die Nacht lieben / und den Tag scheuen / oder vielmehr die Nacht vor den Tag halten. ${ }^{125}$

Hunold ordnet seine vier populären und sensationshungrigen Romane als jugendliche Verirrungen ein. Wenn er die Frühphase seines Schaffens durch die Hervorhebung seines übermäßigen Ehrgeizes und seiner mangelnden moralischen und akademischen Bildung abwertet und in der Retrospektive betont, er habe einen falschen literarischen Weg eingeschlagen, so setzt er seine auf Vergnügen zielenden Romane in ein oppositionelles Verhältnis zu Tugend und Wissenschaft, was der allgemeinen Haltung der Gelehrten zu Beginn des 18. Jahrhunderts entspricht.

Neben seinen akademischen Schriften betätigte sich Hunold als Herausgeber der prestigeträchtigeren Gattung der Lyrik. Er wechselt demnach das Genre, verfolgt jedoch weiterhin eine literarische Betätigung. Dabei bleibt das Ziel der Lesbarkeit zwar relevant, aber es erhält einen neuen Kontext. In der Vorrede zu Auserlesene und teils noch nie gedruckte Gedichte unterschiedener berühmten und geschickten Männer (1717) formuliert er dieselbe Strategie, mit der Defoe in Robinson Crusoe operiert: Die Hauptfunktion der Gedichte sei es, '[d]aß die Poesie ihre Liebhaber auf einem breiten und angenehmen Wege zur Tugend und zur Weisheit führe'. ${ }^{126}$ Hunold benennt eine vorrangige didaktische Funktion, die dem Leser jedoch keine Rezeptionsschwierigkeiten bereiten soll. Dies soll durch eine Kombination mit dem Vergnügen erreicht werden: 'es muß ein vernünftiger Leser auf diesem Wege, wo er Besserung für sein Gemüth und Unterricht für seinen Verstand antrifft, zur Tugend und Weisheit auf eine leichte und vergnügte Manier gelangen'. ${ }^{127}$ Hunold koppelt Vergnügen und Didaktik an Leichtigkeit, wodurch er eine Zusammenführung von docere und delectare vollzieht, bei der das delectare dazu dient, die Hauptwirkungsabsicht des docere durch eine für den Leser vergnügliche Textgestaltung zu fördern. ${ }^{128}$ Das delectare ist demnach dem docere untergeordnet.

\footnotetext{
125 Zitiert nach Hunold 1973, S. 8*.

${ }^{126}$ Hunold 1991, S. 3.

127 Ebd., S. 4.

128 Diese Strategie analysiert Kohl am Bild des 'Zuckerns'; vgl. Kohl 2007 b, u.a. S. 291 und 645.
} 
Auch wenn Hunold in etwa ab dem zweiten Jahrzehnt des 18. Jahrhunderts einen neuen literarischen Schwerpunkt setzte, so standen doch in der öffentlichen Wahrnehmung weiterhin seine Romane im Vordergrund. In der ersten Ausgabe der Literaturzeitschrift Der Teutsche Merkur (1773-1789) verknüpft Christoph Martin Wieland ein auf Breitenwirkung zielendes Lesbarkeitsprogramm mit einem Qualitätsanspruch, wobei er Hunolds Romane hinsichtlich ihrer Qualität energisch kritisiert. ${ }^{129}$ Wieland bettet seine Kritik in eine allgemeine Diskussion der Literaturrezension ein und inthronisiert das zeitgenössische Publikum als oberste Instanz der Literaturbewertung. Dementsprechend etabliert er für den Merkur die Veröffentlichungspraxis, dass '[wir] ohne einige Rücksicht auf Personen und besondere Verhältnisse, den Vorteil des Publikums ganz allein zu Rate ziehen werden'. ${ }^{130}$ Der Publikumsgeschmack bestimmt demnach die Selektion der Texte. Als Grundsatz führt Wieland an, dass es das Ziel der Veröffentlichungen im Merkur sei, 'den Verstand denkender oder das Herz empfindsamer Leser zu unterhalten'. ${ }^{131}$ Wieland positioniert diese Forderung in Ablehnung von Ernsthaftigkeit und Langeweile. Unterhaltung wird zum Desiderat, das Wieland an Verstand und Herz koppelt, was sowohl eine Bildungsfunktion als auch eine emotional bewegende Funktion der Texte miteinschließt, um möglichst viele Leser anzusprechen. ${ }^{132}$ Ebenso geht mit der Forderung nach Unterhaltung ein Qualitätsanspruch einher: 'so haben wir uns zum Gesetze gemacht, allem, was sich nicht in seiner Art über das mittelmäßige erhebt, den Ausschluß zu geben.' ${ }^{\text {133 }}$ Unterhaltung und Qualität stellen für Wieland keine Antipoden dar.

Gerade hinsichtlich der Qualität sah sich Wieland gezwungen, auf eine große Erwartungshaltung der Leserschaft, die vom Merkur ausschließlich 'Meisterstücke' verlangte, zu reagieren. ${ }^{134}$ Als Mittel etabliert er die Strategie, ein möglichst breites und vielfältiges Publikum mit verschiedenartigen Texten erreichen zu wollen:

\footnotetext{
129 Vgl. Wielands 'Vorrede des Herausgebers' in Wieland 1997f, Bd. 2, S. 895-904. Zu Hunold heißt es: 'in Vergleichung mit [...] Menantes mußten Neukirch und Gottsched Virgil und Horaz sein; so wie die gleichgültigste Figur in einer Gesellschaft von hässlichen Zwergen zur Schönheit wird' (Ebd., S. 901). Hunold wird durch die ZwergenAnalogie diffamiert. Hauptsächlich bedauert Wieland jedoch die literarische Unmündigkeit des Publikums.

${ }^{130}$ Ebd., S. 896.

131 Ebd., S. 898.

132 Die Belehrung soll, wie Wieland in 'Der Herausgeber an das Publikum' darlegt, keineswegs 'schulmeisterlich' erfolgen, was auf eine abgeschwächte hierarchische Überlegenheit des Autors gegenüber dem Publikum verweist. Vgl. ebd., S. 905-910.

133 'Vorrede des Herausgebers', ebd., S. 897.

134 'Der Herausgeber an das Publikum', ebd., S. 904.
} 
Auch kann nicht alles gleich gut sein, und nicht alles Allen gefallen. Der Gäste, die der Merkur $\mathrm{zu}$ befriedigen hat, sind sehr viele, und von sehr verschiedener Beschaffenheit. Die einen fordern eine einfache, die andern ein künstlichere Zubereitung; einige [...] haben schwere, andere leichte Speisen vonnöten. Unsre Leser müssen also billig genug sein zu denken, daß man für keinen unter Ihnen allein schreibt und sammelt: oder die Verfasser ziehen ihren Schild wieder ein, und koche wer da will! Alles, wofür ich stehen kann, ist, daß wir Ihrem Geist und Herzen lauter gute, gesunde Nahrung vorsetzen, und sie von Zeit zu Zeit mit einer oder zwoen deliziösen Schüsseln bewirten werden. ${ }^{135}$

Wieland greift auf eine kulinarische Metapher zurück, um das Verhältnis zwischen einfacher und komplexer Literatur zu erläutern. Auffällig ist, dass Wieland zwar für das Einfache plädiert, es jedoch eindeutig in Opposition zum Künstlerischen positioniert. Ein breites Publikum soll nicht durch mittlere Texte gewonnen werden, sondern die Gesamtkonzeption des Merkurs soll einfache und schwere Texte kombinieren. Der Mittelweg wird nicht innerhalb eines Textes, sondern durch ein Prinzip der Abwechslung angestrebt, bei dem einfache und komplexe Beiträge alternieren, um möglichst viele Geschmäcker zu befriedigen.

Wielands Verlangen nach Breitenwirkung äußert sich zudem im Streben nach ökonomischem Erfolg. In Der Herausgeber an das Publikum (1777) beklagt er sich darüber, dass die Qualität seiner Zeitschrift unter zu niedrigen Absatzzahlen zu leiden habe. Deswegen könne er es sich nicht leisten, die besten Autoren für den Merkur zu verpflichten. ${ }^{136}$ Wieland ist sich bewusst, dass diese Haltung im damaligen Literaturbetrieb heftigen Widerspruch ernten musste, und führt, indem er den Standpunkt seiner Gegner ironisch entlarvt, die Debatte selbst ein: 'ihr [Wieland] bekennt uns also [...], daß ihr ums Geld schreibt, und daß ihr euch einbildet, die besten Köpfe zu eurer Fabrik interessieren zu können, wenn ihr sie bezahlen könntet? [...] Welche Niederträchtigkeit!' ${ }^{137}$ Wieland strebt finanziellen Erfolg an, da er glaubt, erst durch ihn hohen Ansprüchen gerecht werden zu können. Zudem befürwortet er, dass die qualitativ hochwertigsten Bücher am häufigsten verkauft werden sollten. ${ }^{138}$ Deutlich wird, dass Wieland bei seinen Gegnern die Abneigung gegen Verkaufserfolg

\footnotetext{
135 Ebd.

136 Andererseits betont Wieland jedoch, dass der Teutsche Merkur auch dazu gegründet wurde, um jungen Autoren eine Plattform zur Präsentation ihrer Werke zu geben; vgl. 'Vorrede des Herausgebers', ebd., S. 895.

137 'Der Herausgeber an das Publikum', ebd., S. 906f.

138 Er moniert, dass der Markt überschwemmt wird und sich oftmals reißerische Titel oder durch inadäquate Rezensionen überhöhte Werke gegenüber den 'besten' Büchern durchsetzen; vgl. 'Neue Bücher', in ebd., S. 911- 914, hier: S. 912.
} 
voraussetzt. Der Merkur wird, um den monetären Charakter zu betonen, zur Fabrik degradiert; dies mündet in die Empörung darüber, die besten Autoren mit Geld gewinnen zu können, was auf das Berufsethos eines nicht von pekuniären Fragen geprägten Schriftstellertums verweist und diesem ein alternatives Berufsbild entgegenstellt. ${ }^{139}$ Wieland beschließt seine Ausführungen mit einer Analogie zu handwerklichen Berufen und etabliert das Schriftstellertum als Arbeit, die angemessen entlohnt werden und den Autoren den Lebensunterhalt sichern müsse. Die Attitüde einer Ablehnung des Geldverdienens durch Literatur sieht Wieland als Ursache für Qualitätsverluste an, da sie die besten Autoren dazu nötige, 'von Bedienstungen zu leben, und ihre beste Zeit mit einträglichern, vielleicht ihrer nicht würdigen Arbeiten zuzubringen und Talente darüber schlafen und verrosten $\mathrm{zu}$ lassen'. ${ }^{140}$ Wieland dekonstruiert eine qualitative Stigmatisierung von finanziell erfolgreicher Literatur und etabliert den Verkaufserfolg als Desiderat und Qualitätsgarant.

Wielands Akzeptanz von finanziellen Ambitionen sollte sich in intellektuellen Kreisen allerdings nicht durchsetzen - vielmehr stellte sich Verdruss über eine mutmaßliche Überschwemmung des Literaturmarktes ein, den auch Wieland anführte. Im Literaturbetrieb wurden Stimmen laut, die eine vermeintliche Lesewut des Publikums einerseits und eine vermeintliche Vielschreiberei der Autoren andererseits verurteilten. Die gesellschaftliche Meinung schlug nach Kiesel und Münch dahingehend aus, dass 'die Literatur durch die ökonomischen Prinzipien des literarischen Marktes einen Qualitätsverlust erlitten habe'. ${ }^{141}$ Dies steht im Gegensatz zu Wielands Theorie, dass die Literatur durch jene Prinzipien eine qualitative Steigerung erfahren würde. Kiesel/ Münch verweisen auf das Negativbild eines professionellen Schriftstellers, der 'in den geheiligten Bereich der Wahrheit und der Ideen mit kommerziellen Absichten eindringt'. ${ }^{142}$ Erfolgreiche Autoren wurden demnach negativ bewertet, wobei sich bereits Anklänge an die Vorstellungen des Idealismus abzeichnen, der seinen Qualitätsbegriff rein über die Ästhetik formuliert. Zudem erhebt die an Goethes

\footnotetext{
139 Der etymologische Duden setzt für den Zeitpunkt der Verwendung des Begriffs in der heutigen Bedeutung 'gewerblicher, mit Maschinen ausgestatteter Produktionsbetrieb' das 18. Jahrhundert an, vgl. Drosdowski 2013, S. 170. Die negative Verwendung des Begriffs im literarischen Kontext ist im Grimm'schen Wörterbuch belegt: 'verächtlich heiszt es bücherfabrik' (Grimm/Grimm 1884, Bd. 3, Sp. 1217).

140 'An das Publikum' in Wieland 1997f, Bd. 2, S. 908.

141 Kiesel/Münch 1977, S. 155.

142 Ebd., S. 91.
} 
Einschätzung erinnernde Sakralmetapher den Schriftsteller in eine prophetische Stellung, die ihn gegenüber dem Publikum exponiert.

In dieser Phase sorgte man sich wie dann später im Literaturstreit von 1990 um die Zukunft der deutschen Literatur. Mit der Annahme, dass sich die literarische Qualität verschlechtert habe, bildete sich eine höhenmetaphorische Bewertungsskala zur Einordnung von Werken heraus. ${ }^{143}$ Ward schlägt für die damalige Rezeption von Romanen eine dreiteilige Einordnungsskala vor, die zwischen Trivialliteratur, Unterhaltungsliteratur und Hochliteratur unterscheidet. ${ }^{144}$ Unterhaltungsliteratur besetzt hier eine von der Trivialliteratur unterschiedene mittlere Position. Unterhaltung wurde zwar akzeptiert, jedoch nicht als Wert an sich; das docere und das movere wurden ihr vorgezogen. Lessing etwa plädiert in der Hamburgischen Dramaturgie (1767-1769) für das docere: der gute Schriftsteller müsse sich zum 'Pöbel [herablassen], [...] um ihn zu erleuchten und zu verbessern. ${ }^{145}$ In der Höhenmetaphorik des Sich-Herab-Lassens steht eine didaktische Funktion im Mittelpunkt, die dem pädagogischen Impetus der Aufklärung entspricht und das delectare als Mittel zur erfolgreichen Umsetzung des Erziehungsauftrags aufwertet. Schiller präferiert in 'Ueber naive und sentimentalische Dichtung' (1795) das movere, indem er Voltaire attestiert, dass dieser zwar als witziger Kopf belustigen, aber nicht als Dichter bewegen könne. ${ }^{146}$ Es ergibt sich zum einen ein Spannungsfeld zwischen den einzelnen Wirkungsabsichten und zum anderen ein Spannungsfeld zwischen diesen und der Lesbarkeit. Während Unterhaltung nur in Kombination mit einer der beiden anderen Funktionen legitimiert ist, erscheint Lesbarkeit kontextabhängig und somit - an den jeweils verfolgten Zweck gebunden als mit allen Wirkungsabsichten vereinbar.

Schiller strebt in seiner ersten Literaturzeitschrift, dem Wirtembergischen Repertorium (1781), ebenfalls eine Fusion von Didaktik und Unterhaltung an und erweitert die Erziehungsfunktion im Vergleich zu Wieland um die literarische Erziehung des Publikums. Die Beiträge sollen zunächst dermaßen gestaltet sein, dass sie 'die

\footnotetext{
143 Bereits Wieland echauffiert sich in 'Neue Bücher' darüber, dass die Qualität der massenhaft veröffentlichten Prosa und Lyrik so schlecht sei, dass man kaum Autoren finde, die nicht in die 'Trivial-Schule' zu senden wären; vgl. Wieland 1997f, Bd. 2, S. 911.

144 Vgl. Ward 1974, S. 57.

145 Vgl. Lessing 1985, Bd. 6, S. 191.

146 Vgl. 'Ueber naive und sentimentalische Dichtung' in Schiller 1943-2012, Bd. 20. S. 413-503, hier: S. 448.
} 
Aufmerksamkeit des größten Teils der Lesenden verdienen'. ${ }^{147}$ Ebenso wie Wieland geht es Schiller darum, seine Leserschaft anzusprechen und zufriedenzustellen, was er dann folgendermaßen zu nutzen gedenkt: 'Unsre Hauptabsicht mit dieser neuen periodischen Schrift ist Ausbildung des Geschmacks, angenehme Unterhaltung und Veredlung der moralischen Gesinnungen'. ${ }^{148}$ Schiller macht wie Wieland eine didaktische und eine unterhaltende Funktion geltend, strebt jedoch explizit nach einer Ausbildung des Publikumsgeschmacks, dessen Unausgegorenheit Wieland lediglich kritisiert hatte, ohne einen Erziehungsanspruch zu formulieren.

Die Affinität zur Leserschaft bekräftigt Schiller in der Thalia (1781), in deren Ankündigung er es als Hauptabsicht begreift, 'zwischen dem Publikum und mir ein Band der Freundschaft zu knüpfen'. ${ }^{149}$ Allerdings distanziert er sich dezidiert von finanziellen Interessen, während Wieland offen dafür eingestanden war, mit dem Merkur Geld erwirtschaften zu wollen. Schiller kritisiert wie Wieland eine Überschwemmung des deutschen Marktes, in Folge derer das Publikum oft von den 'Spekulationen eines Kaufmanns' getäuscht worden sei, der thematische und ästhetische Versprechen nicht eingehalten habe, um seine Verkäufe zu steigern. ${ }^{150}$ Als Reaktion versucht Schiller, die Leserschaft durch eine ausführliche Darlegung des Programms zu gewinnen:

Die Rheinische Thalia wird jedem Gegenstand offen stehen, der den Menschen im allgemeinen interessieret und unmittelbar mit seiner Glückseligkeit zusammenhängt. Also alles, was fähig ist, den sittlichen Sinn zu verfeinern, was im Gebiete des Schönen liegt, alles, was Herz und Geschmack veredeln, Leidenschaften reinigen und allgemeine Volksbildung bewirken kann, ist in ihrem Plane begriffen. ${ }^{151}$

Aus dem Programm des Repertoriums bleiben der Fokus auf die Befriedigung des Publikums, die didaktische Funktion und die Ausbildung des Geschmacks der Leserschaft erhalten. Von einem Unterhaltungsaspekt ist nicht mehr die Rede. Hinzukommen das Ansprechen und kathartische Reinigen von Leidenschaften, das ob deren Wurzeln in der prestigeträchtigen Gattung der Tragödie als Indikator für einen

\footnotetext{
147 'Wirtembergisches Repertorium. Vorbericht' in ebd., Bd. 22., S. 73f, hier: S. 73.

148 Ebd.

149 'Rheinische Thalia und Thalia. Ankündigung', in ebd., S. 93-98, hier: S. 98.

150 Vgl. ebd., S. 93.

151 Ebd., S. 95; das Adjektiv 'rheinisch' wurde nach der ersten Ausgabe gestrichen, was mit der sich verändernden Konzeption der Zeitschrift korrespondiert. Zunächst hatte Schiller die Thalia wie das Wirtembergische Repertorium als Lokalzeitung begriffen, dann jedoch seine Adressatenschaft umfassender aufgefasst.
} 
qualitativen Anspruch angesehen werden kann, der in der idealistischen Kategorie des Schönen seinen Niederschlag erfährt.

In der Ankündigung der Horen setzt Schiller hauptsächlich auf intellektuelle Qualität und engagiert einige der prominentesten Gelehrten seiner Zeit, um durch deren Qualitäten die zersplitterten Leserkreise eines zersplitterten Deutschlands in einem großen Publikum zu vereinen. ${ }^{152}$ Die Einladung zur Mitarbeit (1794) enthält folgenden Aufruf, der die Gelehrten für die Horen gewinnen sollte:

\begin{abstract}
Alles, was entweder blo $ß$ den gelehrten Leser interessieren oder was bloß den nichtgelehrten befriedigen kann, wird [von der Zeitschrift] ausgeschlossen sein; vorzüglich aber und unbedingt wird sie sich alles verbieten, was sich auf Staatsreligion und politische Verfassung bezieht. [...] [I]ndem man bemüht sein wird, die Wissenschaft [...] zu bereichern, hofft man zugleich den Kreis der Leser durch die Form zu erweitern. ${ }^{153}$
\end{abstract}

Schiller, der noch im Sturm und Drang politisch klar gegen die Vorherrschaft des Adels Stellung bezogen hatte, spricht sich gegen ein politisches oder religiöses Engagement aus. Er verlagert den Fokus - mit Ausnahme der wissenschaftlichen Ideen - zunehmend auf formelle Aspekte, die er als Schlüssel zur Gunst des Publikums ansieht. Dabei strebt er eine textuelle Mischform an, die sowohl die gelehrte Elite als auch weniger gebildete Gesellschaftsschichten anspricht. Dabei setzt Schiller wie Wieland auf eine thematische 'Mannigfaltigkeit', jedoch unterscheidet die beiden, dass Schiller auch die Einzeltexte für unterschiedliche Bildungsstände kompatibel konzipiert. ${ }^{154}$

In der Ankündigung (1794) führt Schiller erneut den Programmpunkt der vollkommenen Zurückhaltung in politischen Fragestellungen an und setzt ihn in Bezug zur Unterhaltung:

Einer heitern und leidenschaftfreien Unterhaltung soll sie gewidmet sein, und dem Geist und Herz des Lesers, den der Anblick der Zeitbegebenheiten bald entrüstet, bald niederschlägt, eine fröhliche Zerstreuung gewähren. [...] [Sie] wird [...] an dem stillen Bau [...] edlerer Sitten, von dem zuletzt alle wahre Verbesserung des gesellschaftlichen Zustands abhängt, nach Vermögen geschäftig sein. ${ }^{155}$

\footnotetext{
152 Unter anderen konnte Schiller Johann Wolfgang Goethe, Johann Gottlieb Fichte, Johann Gottfried Herder sowie Wilhelm und Alexander von Humboldt für sein Projekt gewinnen; vgl. 'Ankündigung. Die Horen, eine Monatsschrift, von einer Gesellschaft verfaßt und herausgegeben von Schiller', in ebd., S. 106-109, hier: S. 108f.

153 'Die Horen. Einladung zur Mitarbeit', in ebd., S. 103-105, hier: S. 103.

154 Zur Mannigfaltigkeit siehe ebd., S. 105.

155 'Ankündigung', ebd., S. 106f.
} 
Schiller entwirft sein Programm vor dem Hintergrund des Koalitionskrieges der deutschen Staaten gegen Napoleon, angesichts dessen er ein eskapistisches Zerstreuungsprogramm postuliert, das durch Unterhaltung den Intellekt sowie die Emotionen der Leser ansprechen und sie weg von den realen Umständen des Krieges in einen abgeschlossenen Bereich der Kunst führen soll. ${ }^{156}$ Unterhaltung akzeptiert er in Kombination mit einer didaktischen Wirkungsabsicht. Zudem finden sich Anklänge an das movere, die ebenfalls Unterhaltung legitimieren.

Neben dem Unterhaltungsaspekt führt Schiller in der Einführung zu den wissenschaftlichen Arbeiten der Horen ein leichtes Textverständnis als Mittel zur Publikumsgewinnung ein: 'So weit es tunlich ist, wird man die Resultate der Wissenschaft von ihrer scholastischen Form zu befreien und in einer reizenden, wenigstens einfachen, Hülle dem Gemeinsinn verständlich zu machen suchen'. ${ }^{157}$ Es ist Schillers Anliegen, wissenschaftliche Inhalte möglichst wenig schulmeisterlich und zugänglich für das Publikum zu gestalten, was er mit Wieland teilt. Eine leichte Verständlichkeit ist aufgrund des formulierten Bildungsauftrags für breite Bevölkerungsschichten ein zentrales Desiderat. Allerdings geht es Schiller in seinen Texten nicht um pure Verständlichkeit, sondern diese muss zudem einen ästhetischen Mehrwert aufweisen, um das Publikum anzusprechen.

Schiller beschließt seine Ankündigung mit einer Reflexion über die titelgebenden Göttergestalten der Horen-Schwestern und folgert aus der Sage, dass es sich um eine reizende Dichtung handle, 'durch welche angedeutet wird, daß das Schöne schon in seiner Geburt sich unter Regeln fügen muß und nur durch Gesetzmäßigkeit würdig werden kann, einen Platz im Olymp, Unsterblichkeit und einen moralischen Wert zu erhalten'. ${ }^{158}$ Schiller bindet das Schönheitsdesiderat untrennbar an formelle Aspekte. Ästhetische Regeln müssen strikt befolgt werden, um die Qualität eines Werkes zu garantieren. Ästhetische Qualität avanciert zum wichtigsten Gesichtspunkt der Literatur

\footnotetext{
${ }^{156}$ Es stellt sich die Frage, inwieweit Schillers Postulat einer 'fröhlichen Zerstreuung' mehr als Marketingstrategie denn als tatsächliche Programmaussage zu verstehen ist. Denn im 9. Brief Über die ästhetische Erziehung des Menschen, der in der ersten Ausgabe der Horen erschien, zeigt sich eine deutliche Spannung: 'Der Künstler ist zwar der Sohn seiner Zeit, aber schlimm für ihn, wenn er zugleich ihr Zögling oder gar noch ihr Günstling ist.' Diese Aussage schließt eine vollkommene Anpassung an den Publikumsgeschmack aus. Schiller spezifiziert dann, dass der Künstler es nicht unternehmen soll, '[sein Jahrhundert] mit seiner Erscheinung zu erfreuen, sondern [...] um es zu reinigen.' Schiller lehnt Vergnügen strikt ab und postuliert eine kritische Auseinandersetzung mit den Zeitgegebenheiten, die in eine kathartische Wirkung münden soll; 'Ueber die ästhetische Erziehung des Menschen in einer Reihe von Briefen. Neunter Brief', in ebd., Bd. 20, S. 332-336, hier: S. 333.

157 Ebd.

158 'Ankündigung', ebd., S. 108.
} 
überhaupt und fungiert als Grundvoraussetzung für den moralischen Wert eines Werkes, worin sich das im Begriff der 'Gesinnungsästhetik' enthaltene Spannungsfeld spiegelt.

Schillers erste eigene Horen-Veröffentlichung, die 'Briefe über die ästhetische Erziehung des Menschen' (1795), deuten darauf hin, dass er entgegen dem liberalen Programm in der tatsächlichen Umsetzung überambitioniert gewesen zu sein scheint und er es nicht vermochte, sich die gewünschte breite Leserschaft zu erschließen. ${ }^{159}$ Schillers Misserfolg ist darauf zurückzuführen, dass es ihm nicht gelungen ist, Lesbarkeit mit einem hohen Qualitätsanspruch zu vereinen. Demnach kann Lesbarkeit als valides Mittel zur Breitenwirkung gelten, während eine hohe Komplexität dieser entgegenwirkt.

In der Einleitung in die 'Propyläen'(1798) zeichnet sich ab, dass in Goethes Zeitschrift Autorambition und Publikumsgeschmack noch weiter auseinander driften und sich immer deutlichere Züge des Idealismus finden. Zwar postuliert Goethe, möglichst nützlich und angenehm schreiben zu wollen, steht dem Ruhm, der aus einer Anpassung an den Publikumsgeschmack resultiere, jedoch skeptisch gegenüber; es sei legitim, für das zeitgenössische Publikum zu schreiben, allerdings führten der Ruhm des Schriftstellers sowie die Begeisterung des Publikums zu einer Degeneration der Literatur. ${ }^{160}$ Deswegen unternimmt er den Versuch, die Künstler zu einem Kunstideal auszubilden, das nicht von äußeren Einflüssen geprägt ist, sondern 'ganz auf sich selbst [ruht]'. ${ }^{161}$ Goethes Blick richtet sich auf eine maßgeblich von klassischen Vorbildern inspirierte Ästhetik. Durch die Orientierung an klassischen Vorgängern sollen 'vollkommene Originale' entstehen; diese sollen bewirken, dass, 'begleitet von Kenntnis, Regelmäßigkeit, Ernst und Strenge [...] die Kunst bis zum höchsten hinaufstieg, wo es denn zuletzt dem glücklichen Genie, das sich von allen diesen Hülfsmitteln umgeben fand, möglich ward, das Reizende, Vollendete hervorzubringen'. ${ }^{162}$ Goethe strebt höchste Qualität in Form ästhetischer Vollkommenheit an, die durch die Einhaltung strenger klassischer Regeln sowie das Genie des Autors erreicht werden soll. Die Metapher des Hinaufsteigens und die

\footnotetext{
159 Herbert Meyer schreibt, dass ein Großteil des Publikums Schillers Ausführungen nicht verstanden habe und Schiller daher versucht habe, die Beiträge zu popularisieren, was jedoch die beitragenden Autoren das Interesse an den Horen verlieren ließ. Vgl. Meyer 1958, S. 340.

${ }^{160}$ Vgl. 'Einleitung in die "Propyläen"” in Goethe 1985-1999, Abteilung 1, Bd. 18, S. 457-475, hier: S. 458.

161 Ebd., S. 467.

162 Ebd., S. 471 bzw. 472.
} 
Exponierung des Autors zum Genie suggerieren, dass Autor und Werk durch qualitative Höhen und eine überlegene Bildung vom Leser isoliert werden.

Die Trennung zwischen Autor und Publikum intensivieren August Wilhelm und Friedrich Schlegel in der Vorerinnerung der ersten Ausgabe des Athenäum (1798) durch Höhenmetaphorik: 'für die Unterhaltung aller Leser wünschen wir so viel anziehendes und belebendes in unsre Vorträge zu legen, als ernstere Zwecke erlauben'. ${ }^{163}$ Die Schlegels stellen Unterhaltung und Ernsthaftigkeit einander gegenüber, wobei 'ernstere Zwecke' eine privilegierte Stellung einnehmen. Unterhaltung sehen sie als Mittel zur Lesergewinnung, die sie allerdings als Sekundärziel etablieren. Zudem kann Unterhaltung als Schmälerung der ernsthaften Absichten, die einen Qualitätsverlust nach sich zieht, gelten.

Im Hinblick auf Qualitätshierarchien unterscheidet Friedrich Schlegel in Über das Studium der Griechischen Poesie (1797) zwischen einer 'höhern und niedern Kunst':

Sie [die Poesien] nehmen nicht die geringste Notiz voneinander, außer wenn sie zufällig aufeinander treffen, durch gegenseitige Verachtung und Spott; oft nicht ohne heimlichen Neid über die Popularität der einen oder die Vornehmigkeit der andern. Das Publikum, welches sich mit der gröbern Kost begnügt, ist naiv genug, jede Poesie, welche höhere Ansprüche macht, [...] von der Hand zu weisen. ${ }^{164}$

Schlegel rekurriert auf eine Höhenmetapher und eine kulinarische Metapher. Die 'niedere' Poesie setzt er mit 'grober' Kost gleich und verwendet die Metapher wie Wieland zur Beschreibung der Machart. Während Wieland die Schwierigkeit der Rezeption ausdrückt, nutzt Schlegel die Metapher zur Unterscheidung der Kunstfertigkeit der beiden Poesien. Er verbindet die 'niedere' Poesie mit großem Publikumserfolg, den die 'höhere' Poesie aufgrund ihrer qualitativ hochwertigen Gestaltungsweise nicht erreichen kann, was die bereits unternommenen Beobachtungen zu Schillers Horen bestätigt. Erfolg und Qualität fungieren demnach als entscheidende Distinktionsmerkmale der sich exkludierenden Poesien, wobei Schlegel die 'naiven' Leser, wegen ihrer Zurückhaltung bei komplexen Formen kritisiert.

Allerdings wurden in Gelehrtenkreisen widersprechende Stimmen laut, die für die Lesebedürfnisse eines wenig gebildeten Publikums eintreten. Ludwig Tieck sieht es in

\footnotetext{
163 Schlegel/Schlegel 1924, S. 4.

${ }^{164}$ Schlegel 1958-, Erste Abteilung. Bd. 2, S. 217-367, hier: S. $226 f$.
} 
'Kritik und deutsches Bücherwesen. Ein Gespräch' (1828) als selbstverständlich an, wenn 'der Mensch sich [...] auf Genuß an sich selbst zurückzieht, und daß er sich in seinen müßigen Stunden von feinen Romanzen ergötzen läßt.'165 Tieck erkennt das Vergnügen als gerechtfertigten Lektürezweck an und legitimiert dadurch auf Unterhaltung zielende Werke. Da er das Vergnügen als Hauptanspruch der Leserschaft an die Literatur ansieht, muss dieses Verlangen, 'wenn es ein wahres Bedürfnis ist, befriedigt werden'.166 Tieck verpflichtet die Autoren darauf, die Vorlieben der Leserschaft zu berücksichtigen, und wertet so die Position des Lesers auf.

Georg Wilhelm Friedrich Hegel als Hauptvertreter des philosophischen Idealismus wendet hingegen in seinen zwischen 1817 und 1829 gehaltenen Vorlesungen über die Ästhetik das Augenmerk vollkommen auf die Ästhetik und schließt die Publikumswirksamkeit von sämtlichen Überlegungen aus. Unter der Prämisse des 'freien poetischen Kunstwerks' äußert er in Bezug auf die Wirkungsabsichten: '[D]ie Poesie [muss] sich vor jedem außerhalb der Kunst und des reinen Kunstgenusses liegenden Zweck bewahren'. ${ }^{167}$ Er lehnt textexterne Zwecke, die Literatur mit einer Funktion gegenüber der realen Welt versehen, was die Unterhaltung ebenso wie die Erziehung des Lesers miteinschließt, als Funktionen ab, die der 'poetischen Schönheit' entgegenwirken. ${ }^{168}$ Dies begründet er dadurch, dass 'in der Dichtkraft nur das Poetische, nicht aber das, was außerhalb der Poesie liegt, als bestimmender und durchgeführter Zweck regieren' dürfe. ${ }^{169}$ Literatur ist demnach einzig ihrem ästhetischen Regelwerk verpflichtet, welches als anzustrebendes Ideal fungiert. Sollten Texte sich dennoch nach einem textexternen Zweck richten, so habe dies zur Folge, dass ein Werk 'aus der freien Höhe in deren Region es nur seiner selbst wegen dazusein [sich] zeigt' heruntergezogen wird und zu einem Mittel der 'Zweckdienlichkeit' verkommt. ${ }^{170}$ Hegel stellt die Unabhängigkeit als Grundvoraussetzung seines Literaturbegriffs dar: Sobald sich Literatur nicht ausschließlich um ihre ästhetischen Werte dreht, bemüht Hegel die Höhenmetapher des Herunterziehens, um ihren

\footnotetext{
165 Tieck 1848-1852, Bd. 2, S. 133-170, hier: S. 141f.

166 Ebd., S. 142f.

167 Hegel 1986, Bd. 15, S. 266-268, hier: S. 268.

168 Vgl. ebd., S. 268f.

169 Ebd., S. 269.

170 Ebd., S. 268.
} 
Qualitätsverlust auszudrücken, der einem Verlust des literarischen Status gleichkommt.

Während Hegels Literaturbegriff die politischen, gesellschaftlichen und geistesgeschichtlichen Veränderungen im Nachgang zur Französischen Revolution und die einsetzende Industrialisierung in eine idealistische Abschottung von außerästhetischen Einflüssen kanalisierte, bildeten sich zu Beginn des 19. Jahrhunderts als Reaktion auf das politische Geschehen desgleichen engagierte Schriftstellergruppierungen wie das 'Junge Deutschland' heraus. Darüber hinaus schufen die Einführung der Papiermaschine und der Schnellpresse sowie der seit 1835 rapide zunehmende Eisenbahnbau ein leistungsfähiges Kommunikationssystem, das zur Erschließung bisher unerreichter Gegenden für den literarischen Markt und zu einer größeren und schnelleren Verbreitung von Büchern in Deutschland führte. Bei gleichzeitig ansteigendem Bildungsstand bisher bildungsferner Bevölkerungsschichten und gesteigerter Lesefähigkeit durch eine strenger durchgesetzte Schulpflicht standen sowohl die technischen Voraussetzungen als auch eine potenzielle Abnehmerschaft für Bücher zur Verfügung, die die Grundvoraussetzungen für eine zur damaligen Zeit neue Massenliteratur schufen. ${ }^{171}$ Diese Grundlage begünstigte u.a. die zunehmende Beliebtheit von literarischen Zeitschriften und den in ihnen erscheinenden Fortsetzungsromanen.

Dieser Entwicklung des Literaturmarktes vergewissert sich Karl Gutzkow in der Vorrede zu seinen Novellen (1834) und führt dann ein poetologisches Programm als Mittel zur Breitenwirkung ein, in dem er sich grundsätzlich der Lesbarkeit verschreibt. Er beklagt zunächst den Missstand, welchen Wieland mit seinem Merkur zu beheben suchte, dass in Deutschland keine adäquate Plattform existiere, um die Werke junger Autoren einem breiten Publikum vorzustellen. Dennoch appelliert er an den Nachwuchs, sich nicht von den lokalen Begebenheiten einschränken zu lassen, und über die Kleinstaaten hinaus, aktiv den Weg zu einem 'deutschen [Publicum]' zu suchen. ${ }^{172}$

Als Mittel zur Breitenwirkung gibt Gutzkow seinen jungen Kollegen die im 19. Jahrhundert aufstrebende Gattung der Novelle als Erfolgsgarantin zur Hand, die Gutzkow mit dem poetologischen Ansatz versieht, die Texte publikumsfreundlicher zu

${ }^{171} \mathrm{Zu}$ den Auswirkungen der industriellen Revolution auf den Literaturbetrieb des 19. Jahrhunderts siehe Kienzle 1975.

172 Vgl. 'Vorrede' in Gutzkow 2001, Eröffnungsband, S. IX-3 - IX-11, hier: S. IX-5. 
gestalten. ${ }^{173}$ In der Vorrede inszeniert Gutzkow einen Dialog zwischen einem fiktiven Ratgeber und einer Figur, die den Namen Karl Gutzkow trägt. Der Ratgeber nimmt die Perspektive des Publikums ein und greift auf das höhenmetaphorische Bild des 'Herabsteigens' zurück, um der Figur Gutzkow ein neues Verhältnis zum Publikum zu erläutern:

$\mathrm{Du}$ [Gutzkow] gabst Narrenbriefe heraus, welche selbst für Kluge zu gescheut waren. Du schriebst eine Göttergeschichte, und die Welt ist $\mathrm{zu}$ ab- und gegengöttisch, als daß Du Dich damit bei ihr empfehlen könntest. Du brütest schon über neuen Plänen, welche an Unpopularität die frühern noch überbieten dürften. Sieh Dich vor! Verscherze Dein Talent nicht! Zu den Bedürfnissen steige herab, laß Deine Götter Menschen werden, gleich uns! ${ }^{174}$

Gutzkow erkennt einen zu hohen intellektuellen Anspruch bei seinen früheren Werken und bei den Planungen künftiger Werke. Deshalb sei ihm bisher der Publikumserfolg verwehrt geblieben. Um dem entgegenzuwirken, empfiehlt der fiktive Ratgeber ein Umdenken hinsichtlich der Thematik, die er im Sinne Aristoteles' an einer Gestaltung der Figuren festmacht, die dem Leser gleichen sollen. Gutzkow solle sich von seinen hohen Ambitionen lösen und die literarischen Bedürfnisse der Leserschaft bedienen. ${ }^{175}$ Dies wird nicht als negatives Sich-Herablassen auf das Niveau eines niederen Publikums präsentiert, sondern als Mittel, sein Talent nicht zu vergeuden. Talent kann sich demnach erst durch die Popularität bei der Leserschaft endgültig entfalten.

Dennoch hegt Gutzkow qualitative Vorbehalte gegen eine realistische Schreibweise. Er äußert, dass er mit seinen Novellen 'auf der Stufe der ordinären Wirklichkeit [bleibt], welche man nur treu zu schildern braucht, um für genial, unübertrefflich, und in seiner Art einzig gehalten zu werden'. ${ }^{176}$ Die treue Schilderung der Wirklichkeit stellt für Gutzkow demnach keine besondere Fähigkeit dar, wodurch er eine Kritik an der rezensorischen Überhöhung einer solchen Schreibweise übt. Er strebt danach, sich von den Einschränkungen eines realistischen Literaturbegriffs zu befreien: 'Ich trag' [das Publikum] dann, wohin ich will, heraus aus dieser trügerischen Welt,

\footnotetext{
173 Gutzkow spricht sich ebenso für die Unterhaltung des Lesers aus. In seinem Essay 'Der deutsche Roman' (1836) schreibt er, dass es keinen größeren Fluch in der Poesie gebe als die Langeweile; vgl. 'Der deutsche Roman' in Gutzkow 2001.

174 'Vorrede', ebd., S. IX-6f.

175 Zudem formuliert Gutzkow das Ziel, dass ihm die Leser über 'Berg und Tal' folgen sollen. Er rekurriert auf Höhenmetaphorik, um schwierige Stellen zu beschreiben, und nimmt die Funktion eines unterstützenden Begleiters ein, der den Lesern über jene hinweghilft. Ebd., S. IX-8.

176 Ebd., S. IX-7.
} 
deren falsche Bilder ich so lange aufgefangen und wiedergegeben hatte, in die Nähe des Firmaments, in ätherische Regionen, in andere Sphären, Ideen, in Träume'. ${ }^{177}$ Gutzkow sieht die Abkehr von der treuen Abbildung der Wirklichkeit als Mittel, zu künstlerischen Höhen zu gelangen. Gutzkow beschreibt diese Höhen mit teils transzendenten Metaphern, die zum einen für eine Erhabenheit des Geschriebenen und zum anderen für einen genialistischen Originalitätsanspruch stehen, der durch eine treue Abbildung der Wirklichkeit nicht zu erlangen ist.

Gutzkows Ansicht, dass Autoren erst durch den Publikumserfolg ihr Talent vollständig entfalten können, wirkt diesem Geniebegriff entgegen. Der Verbund mit der prestigeträchtigen Genieästhetik fungiert als Legitimationsstrategie für Gutzkows Breitenwirkungsdesiderat und realistischen Literaturbegriff, der in Thematik und Komplexität auf die Leserschaft ausgerichtet sein soll. Demnach ist für Gutzkow Lesbarkeit ein inhärenter Bestandteil eines qualitativ ambitionierteren Literaturbegriffs.

Das Programm von Ernst Keils Die Gartenlaube. Illustriertes Familienblatt (ab 1. Januar 1853) gründete ebenfalls auf einem realistischen Literaturbegriff, formulierte einen dezidierten Unterhaltungsanspruch, der etwa E. Marlitt zu einer der meist gelesenen Autorinnen der Zeit machte und die Gartenlaube einen seinerzeit einzigartigen Siegeszug antreten ließ, wobei sie zu ihrer Blütezeit ästhetisch und thematisch stilbildend auf ein breites Lesepublikum wirkte. Neben ihrer Erschwinglichkeit ist ihr Erfolg vor allem auf ihren Unterhaltungsgrundsatz zurückzuführen, den Keil auf der Titelseite der ersten Ausgabe benennt:

So wollen wir Euch unterhalten und unterhaltend belehren. Über das Ganze [das Blatt] aber soll der Hauch der Poesie schweben wie der Duft auf der blühenden Blume, und es soll Euch anheimeln in unserer Gartenlaube, in der Ihr gut-deutsche Gemütlichkeit findet, die zu Herzen spricht. ${ }^{178}$

Zwar steht das unterhaltende Moment im Mittelpunkt von Keils Ansatz, jedoch erschöpft er sich nicht darin. Wie etwa Wieland kombiniert Keil Unterhaltung mit Belehrung. Die Belehrung soll unterhaltend gestaltet werden, was die Unterhaltung zwar zur primären, aber nicht zur einzigen Wirkungsabsicht werden lässt. Dass Keil 
nicht ausschließlich Unterhaltung anstrebt, zeigt auch die Herzmetaphorik, die auf eine emotional sanft bewegende Funktion der Texte schließen lässt. Abgesehen von den Wirkungsabsichten zeigt Keils Desiderat eines 'Hauch[s] von Poesie', dass der Verleger in sehr zurückgenommener Form einen Qualitätsanspruch erhebt.

Durch Vielseitigkeit versuchte Keil, den Geschmack eines möglichst breiten Publikums anzusprechen. Im Vordergrund standen dabei, wie bereits der Untertitel der Gartenlaube hervorhebt, die Familien:

\begin{abstract}
Wenn ihr im Kreise Eurer Lieben die langen Winterabende am traulichen Ofen sitzt oder im Frühlinge, wenn vom Apfelbaume die weiß und roten Blüten fallen, mit einigen Freunden in der schattigen Laube - dann leset unsere Schrift. Ein Blatt soll's werden fürs Haus und für die Familie, ein Buch für Groß und Klein, für jeden, dem ein warmes Herz in den Rippen pocht, der noch Lust hat am Guten und Edlen! Fern von aller räsonnierenden Politik und allem Meinungsstreit in Religions- und anderen Sachen, wollen wir Euch in wahrhaft guten Erzählungen einführen in die Geschichte des Menschenherzens und der Völker, in die Kämpfe menschlicher Leidenschaften und vergangener Zeiten. ${ }^{179}$
\end{abstract}

Die einleitende Rezeptionsempfehlung begründet den universellen Grundcharakter der Zeitschrift. Neben der Evokation behaglich-biedermeierlicher Rezeptionsszenarien und -orte, die mit dem Bild der Gartenlaube definiert sind, wird die Lektüre in der Gemeinschaft von Familie und Freunden als ideale Rezeptionsform eingeführt. Innerhalb der Zielgruppe der Familie spiegelt sich wiederum die zu gewinnende Leserschaft in der gesamten Gesellschaft. Die Autoren mussten diverse Interessen bedienen, die sich z.B. auf unterschiedliche Generationenzugehörigkeit oder divergierenden Bildungsstand zurückführen lassen. Mit der Lust am 'Guten und Edlen' rekurriert Keil auf Goethes 'Das Göttliche' und eine moralische Haltung und verspricht zudem eine künstlerisch anspruchsvolle Gestaltung. ${ }^{180}$

Es muss in Erwägung gezogen werden, inwieweit das Postulat, sich aus politischen und religiösen Angelegenheiten herauszuhalten, eher als Schutzfunktion denn als tatsächliche Absicht aufzufassen ist. ${ }^{181}$ Beispielsweise verweist Marlitt im Vorwort des Keil gewidmeten und in der Gartenlaube erschienenen Romans Reichsgräfin Gisela (1870) darauf, dass die Gartenlaube im ‘edlen deutschen Wesen' gründet und 'in ihren Blättern, Blühten und Früchten den Segen einer sittlich reinen, von verknöcherten

\footnotetext{
$179 \mathrm{Ebd}$.

180 Vgl. Goethe 1985-1999, Abteilung 1, Bd. 2, S. $303 \mathrm{f}$.

${ }^{181}$ Keil war wegen politischem Aktivismus zu einer mehrmonatigen Haftstrafe verurteilt worden, die zu einem Verbot seiner früheren politischen Publizistik führte, vgl. die Einleitung zu Hamouda 2005.
} 
Dogmen und Formen sich losringenden Weltanschauung ausströmt'. ${ }^{182}$ Dies zeugt eindeutig von einem Engagement für die nationalistische Bewegung und von einer liberalen Weltanschauung. Das politische Wirken der Zeitschrift thematisiert Johannes Proelß in seiner 1902 und 1903 in der Zeitschrift erschienenen 'Geschichte der Gartenlaube'. Wie Proelß, einer der Nachfolger Keils in der Funktion des Herausgebers, unterstreicht, trat die Zeitschrift intensiv für ein geeintes Deutschland ein. ${ }^{183}$ Auch zu religiösen Fragestellungen bezog die Gartenlaube Stellung. Als Beispiel kann Keils 'Brief an eine Gläubige' (1866) gelten, in dem er eine anonyme Bekannte einer falschen Glaubensauslegung und religiös kaschierter Doppelmoral überführt, die auch in der Handlung und der Personencharakterisierung in Marlitts Romanen zum Ausdruck kommt. ${ }^{184}$ Zweifelsfrei kann somit von einem didaktischen Anspruch der Gartenlaube ausgegangen werden. Lesbarkeit dient dazu, möglichst breiten Bevölkerungsschichten eine umfassende Allgemeinbildung zugänglich zu machen.

Um Lesbarkeit sicherzustellen, legt Keil vor dem Erscheinen der ersten Ausgabe ein sechs Punkte umfassendes ästhetisches Programm fest, das hier vollständig zitiert wird, um das komplexe Zusammenspiel der Kategorien herausarbeiten zu können:

1. Gedichte unserer besten Poeten, und zwar stets gut illustriert.

2. Novellen, möglichst kurz mit höchstens 2-3 Fortsetzungen. Ebenfalls illustriert. Die Stoffe der Erzählungen sind stets der Geschichte des Vaterlandes (Lokalnovellen) oder den Zuständen des neuern Volkslebens zu entnehmen. In jeder Nummer wird der Erzählung circa 8 Spalten Raum gegönnt.

3. Schilderungen, besonders interessante, der Sitten, Gebräuche und Zustände deutscher und fremder Völker.

4. Briefe aus der Natur. Irgendeine Persönlichkeit, die noch zu erfinden, bespricht in durchaus populären Briefen die wichtigsten und nächstliegenden Fragen aus dem Naturleben; z.B. das Wasser, die Luft, den Mond, die Pflanzen, die Sterne, Wolken, Bau der Insekten und Käfer, das Eisen, Erz etc., wozu gute Abbildungen geliefert werden. Diese belehrenden Briefe dürfen indes durchaus keinen schulmeisterlichen Anstrich haben, sondern müssen durchweg leicht verständlich, elegant, womöglich in novellistischer Form geschrieben werden, so dass sie die gewöhnlichsten Handwerker, besonders aber die Frauen verstehen können.

5. Der äußere und innere Mensch [...]. Eine Reihe ebenfalls populärer Briefe über den Bau, die Tätigkeit und das Leben des menschlichen Körpers. Mit Abbildungen. Wie in den obigen Briefen das außermenschliche Naturleben behandelt wird, so soll in diesen lediglich der Mensch in seinen einzelnen Teilen und Funktionen (natürlich mit der nötigen Dezenz) geschildert werden. Wenn der Mensch sich ganz klar werden will, so muss er vor allem sich selbst kennen lernen, das Herz, das Auge, die Lunge, das Ohr, das ganze Nervensystem. Alles spricht darüber, aber niemand kennt es. Diese Kenntnis des menschlichen

\footnotetext{
182 Marlitt 1870, S. [VI].

183 Vgl. Proelß 2005, S. 83-97.

184 Vgl. Keil 2005; als Beispiel kann Marlitts Roman Das Geheimniß der alten Mamsell (1867) gelten.
} 
Körpers den weniger Gebildeten spielend und auch auf eine unterhaltende Weise beizubringen ist der Zweck der Abhandlungen und Briefe, die ebenfalls elegant, populär und für jedermann verständlich geschrieben werden müssen.

6. Ein kleines Feuilleton mit Notizen aus der Zeit und der Literatur schließt jede Nummer, indes wird dies immer nur als Lückenbüßer gebraucht. ${ }^{185}$

Keil stellt sowohl der Lyrik als auch der Prosa Zeichnungen zur Seite, deren genaue Funktion er nicht angibt. Zieht man allerdings den engeren Wortsinn heran, so kann auf die Aufgaben einer Veranschaulichung und Deutung des Textinhalts geschlossen werden, die zu einem besseren Verständnis beitragen sollen. Bei der Thematik der Novellen fällt auf, dass Keil auf eine Darstellung speziell deutscher Stoffe pocht - seien sie historisch oder eine Beschreibung des gegenwärtigen Lebens. Letzteres spricht für einen tendenziell realistischen Literaturbegriff, dessen mimetische Darstellung der Lebensbedingungen der Leser die Lesbarkeit fördern soll. Zudem sind die Novellen aufgrund der Publikationsgegebenheiten auf einen limitierten Umfang beschränkt, was das Stilprinzip der brevitas auf der Ebene des Textumfangs spiegelt.

Fiktionale Elemente sollen ebenfalls in die wissenschaftlichen Texte einfließen, mit denen Keil anstrebt, komplexe Fragestellungen für ein breites Publikum verständlich und unterhaltsam darzustellen. Zwar fordert er dezidiert eine didaktische Funktion der Texte ein, jedoch nicht um den Preis der Lesbarkeit. Es sollen nicht auf 'schulmeisterliche[...]' Art Eliten, sondern ein bildungsfernes Publikum angesprochen werden, was durch die Aufgabe der didaktisch erhöhten Position, die sich auch bei Wieland und Schiller findet, zum Ausdruck kommt. Zur Beschreibung der Gestaltungsweise führt Keil Attribute an, die dem Lesbarkeitsbegriff entstammen: 'durchweg leicht verständlich' und 'spielend' stehen für ein leichtes Textverständnis, 'populär und für jedermann verständlich geschrieben' für eine angestrebte Breitenwirkung, 'auf unterhaltende Weise' für eine Favorisierung des delectare als Wirkungsabsicht und 'elegant' für ein Streben nach ästhetischer Qualität. Lesbarkeit ist demnach eine inhärente Qualität der didaktischen Publikationen der Gartenlaube, um ihr Hauptziel einer Förderung der Allgemeinbildung in breiten Bevölkerungsschichten zu erreichen.

Trotz ihres qualitativen und didaktischen Anspruchs sahen sich die Gartenlaube und deren Autoren etlichen abschätzigen Kritikern gegenüber. Marlitt führt die Angriffe 
auf ihr Werk im Vorwort zur Reichsgräfin Gisela an. Sie spricht Keil dafür Dank aus, dass er sie unterstützt habe, 'wenn hie und da eine Hand plump aus mystischem Dunkel nach mir herübergriff und mein Wollen und Wirken $\mathrm{zu}$ verdächtigen suchte'. ${ }^{186} \mathrm{Ihr}$ Wirken stellt Marlitt in den Dienst der Humanität und der Darstellung von moralisch sittlichen Anschauungen. ${ }^{187}$ Diese äußerten sich meist in der Handlung, der Tugend der stets weiblichen Hauptfigur und in der negativen Zeichnung von Figuren, die nichtliberale Anschauungen vertreten. ${ }^{188}$ Dahingehend wurde Marlitts Werken vor allem von ihren männlichen Kollegen oftmals Qualität abgesprochen, die jene als 'Frauenzimmerromane' abqualifizierten. ${ }^{189}$ Während sich Gottfried Keller gegen die Kritiker Marlitts wandte, lancierte Theodor Fontane eine harsche Kritik, aus der ersichtlich wird, dass die Kritik an Marlitt zusehends auch eine Kritik an der Gartenlaube generell darstellte. So verwendet Fontane in einem Brief an Gustav Karpeles (3. März 1881) die Begriffe 'Gartenlaubenstil' und 'alt-überkommener Marlittstil', um beide herabzuwürdigen und spricht es Marlitt ab, eine Schriftstellerin zu sein. ${ }^{190}$ Es geht einerseits um das Verhältnis zwischen der Autorin und ihrem Publikationsmedium, andererseits um die Kompatibilität von finanziell erfolgreichen Werken und ästhetischer Qualität, die einen zentralen Streitpunkt im Lesbarkeitsdiskurs darstellt. Die Gartenlaube sah sich wegen ihres auf eine unterhaltsame und lesbare Literatur zielenden Programms teils heftigen Anfeindungen von Verfechtern eines hohen literarischen Anspruchs ausgesetzt. Doch gerade ihr publikumsfreundlicher Charakter erschloss der Gartenlaube eine breite Leserschaft und ermöglichte es ihr, auf große Teile der Gesellschaft zu wirken.

Theodor Fontane entwirft hingegen in seinem 1853 erschienenen Aufsatz 'Unsere lyrische und epische Poesie seit 1848' die Theorie eines qualitativ hochwertigen poetischen Realismus, der sich jedoch ebenso aus dem Lesbarkeitsbegriff speist. Das

\footnotetext{
186 Marlitt 1870, S. [VI].

187 Im Vorwort schreibt Marlitt in einer Analogie zwischen Gartenlaube und ihrem Roman, dass bei beiden die moralischen Grundsätze an die Menschenherzen rühren und Menschenliebe fördern sollten. Ebenso würden beide gegen jene zürnen, die nur auf ihren eigenen Vorteil aus seien und daher inhumane Standpunkte verträten; vgl. ebd., S. [VII].

188 Katrin Kohl äußert in ihrem Beitrag 'E. Marlitt's Bestselling Novels' (2012), dass das moralische (und oftmals auch an politische Fragestellungen gekoppelte) Anliegen durch das Ansprechen der Emotionen der Leser und das große Identifikationspotenzial maßgeblich zum Unterhaltungswert von Marlitts Romanen beitrug und in diesem Sinne den Grundsätzen der Gartenlaube entsprach; vgl. Kohl 2012.

189 Vgl. Proelß 2005, S. 125.

190 Vgl. Gottfried Kellers Brief an Josef Victor Widmann vom 22. März 1885 in Keller G. 1950, Bd. 1, S. 251; Fontane 1962-1997, Abteilung 4, Bd. 1, S. 120 bzw. 158.
} 
Realismusprogramm sollte es seinen Vertretern ermöglichen, selbst im Vergleich zur Weimarer Klassik, 'eine Blüte unserer Literatur, vielleicht ihre höchste, herbeizuführen'. ${ }^{191}$ Fontane exponiert den Realismus qualitativ durch den Vergleich mit der Tradition. Besonders positioniert er den Realismus als Reaktion auf den 'blühende[n] Unsinn' der Romantik und kritisiert deren vermeintlich thematisch und ästhetisch realitätsferne Literatur. ${ }^{192}$ Fontanes Antagonismus unterstreicht den Wettkampfcharakter des Lesbarkeitsbegriffs, den er dazu verwendet, um sich von einer Vorgängerästhetik abzusetzen und mit ihm eine Verbesserung für die deutsche Literatur herbeizuführen, was er in der Metapher einer 'Wiedergenesung' ausdrückt. ${ }^{193}$

Fontanes Realismusbegriff gründet auf den Lesbarkeitsaspekten der Textverständlichkeit, Unterhaltung und Breitenwirkung sowie auf finanziellem Erfolg, was insbesondere aus seinen Briefen und Tagebüchern hervorgeht. Hinsichtlich der Verständlichkeit schreibt Fontane 1894 in einem Brief über den Schriftsteller Rudolf Lindau: 'Der Auszug aus Lindaus Buch ist sehr interessant und auch rein schriftstellerisch angesehen, wieder ganz vorzüglich: klar, leichtverständlich, anschaulich, graziös'. ${ }^{194}$ Die schriftstellerische Qualität Lindaus macht Fontane anhand von Kategorien fest, die alle aus der Rhetorik stammen und auf eine lesbare und qualitativ ansprechende Textgestaltung hindeuten. 'Anschaulichkeit' ist das Hauptziel bei Quintilians Anmerkungen zur stilistischen Gestaltung, die wiederum auf leichte Verständlichkeit zielt. Die Klarheit im Ausdruck ist ebenfalls ein rhetorisches Stilprinzip, das die Zugänglichkeit für eine diverse Leserschaft sicherstellt. 'Graziös' verweist auf eine anmutige Stilistik, die sich durch den Redeschmuck einstellt.

Ebenso wie Fontane diese Qualitäten bei Lindau lobt, tadelt er deren Nichtbeachtung in anderen Werken wie etwa Leopold von Rankes Weltgeschichte, an deren Stilistik er in einem Tagebucheintrag des Jahres 1886 kritisiert, dass jede Klarheit verlorengeht. ${ }^{195}$ In einem Brief vom 12. April 1894 bemängelt Fontane, dass sich durch die literarische Rezeption der Klassik bei deutschen Schriftstellern ein gewisser 'Zierstil' eingestellt habe, dessen übertriebener Hang zum Redeschmuck erst durch den Einfluss des Naturalismus habe getilgt werden können. ${ }^{196}$ Eine generelle Ablehnung

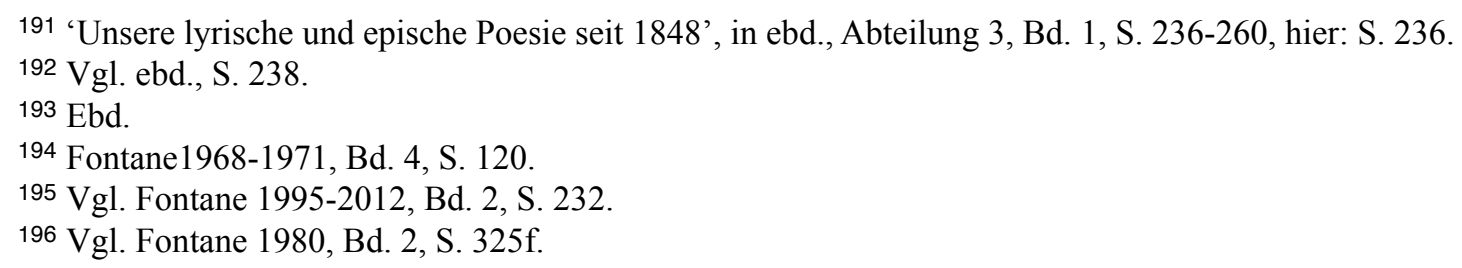


eines schwer zu lesenden Werkes findet sich in einem Brief vom 19. Januar 1889; an einem Text Konrad Albertis kritisiert Fontane: '[D]ie ganze Brochüre [ist] nichts als [...] Quatsch, so unangenehm [...] daß es eine Riesenaufgabe ist, diese $120 \mathrm{~S}$. durchzulesen'. ${ }^{197}$ Christian Friedrich Scherenberg wirft er in 'Unsere lyrische und epische Poesie' vor, dass es jenen nicht kümmere, ob der Leser die Gedanken des Autors nach langem Kopfzerbrechen verstehe, woraus sich ableiten lässt, dass Fontane die Autoren selbst anhält, für die Lesbarkeit ihrer Werke zu garantieren. ${ }^{198}$

Neben einem leichten Textverständnis fordert Fontane auch eine Unterhaltungsfunktion ein, deren Wert er in der deutschen Literaturlandschaft zu seiner Zeit nicht ausreichend gewürdigt sieht. In 'Unsere lyrische und epische Poesie' heißt es hinsichtlich der Rezeption des Autors Ferdinand Freiligrath, dass es charakteristisch für die deutsche Gesellschaft im Allgemeinen geworden sei, über das Werk Freiligraths 'vornehm die Achseln zu zucken und zu tun, als verrate es Mangel an Bildung und Geschmack, sich durch so "gedankenloses Zeug" unterhalten zu lassen.' ${ }^{199}$ Fontanes Kritik richtet sich gegen die Annahme, dass mit einem Bekenntnis zu einer Unterhaltung ohne tiefere Gedankengänge ein Prestigeverlust für den Leser einhergeht und wendet sich demnach dagegen, Unterhaltung einen Eigenwert abzusprechen. Zwei Erkenntnisse sind von Relevanz: Erstens stellt Fontane fest, dass zu seiner Zeit in der deutschen Literaturlandschaft eine Präferenz für Unterhaltung mit einem Mangel an Bildung in Verbindung gesetzt wird. Dies verweist darauf, dass mit der Wahl der Lektüre ein soziales Ansehen einhergeht, bei dem Unterhaltung bildungsfernen Lesern zugerechnet wird, während die Literatur der gebildeten Rezipienten aus komplexeren Gedankengängen zu bestehen hat. Zweitens zieht Fontane wie Wieland und Schlegel mit dem 'Geschmack' eine kulinarische Metapher als Referenzsystem heran. Diese Metapher nimmt er auch auf, wenn er am 7. Juli 1884 schreibt, dass er sich bei der privaten Lektüre hauptsächlich 'von unterhaltlichem Stoff genährt' habe. ${ }^{200}$ Durch die positive Formulierung der Ernährungsmetapher befreit er Unterhaltung nicht nur vom Stigma des schlechten Geschmacks, sondern etabliert sie als anerkannte Lektürefunktion. Dass der Eigenwert von Unterhaltung für Fontane jedoch nicht gleichzeitig deren qualitativem Wert entspricht, zeigt ein Brief vom 7. Februar 1889, in

\footnotetext{
${ }^{197}$ Fontane1968-1971, Bd. 4, S. 104.

${ }^{198}$ Fontane 1962-1997, Abteilung 3, Bd. 1, S. 251.

199 Ebd., S. 245.

${ }^{200}$ Vgl. Fontane 1980, Bd. 2, S. 340 f.
} 
dem sich Fontane für die finanzielle Unterstützung des Schriftstellers Adolf von Winterfeldt ausspricht: 'Ueber seine Bedürftigkeit ist kein Zweifel und wenn sich über seine literarischen Ansprüche vielleicht streiten läßt, so wird doch als Thatsache gelten dürfen, daß er durch seine Soldatengeschichten, ein Menschenalter hindurch, viele Tausende von Lesern erfreut hat.' ${ }^{201}$ Fontane sieht das delectare als zentrale Wirkungsabsicht in Winterfeldts Werk und lobt dies als eigenständiges Verdienst. Auch wenn er Winterfeldt literarische Qualität eher zugesteht, räumt er ein, dass dies diskutabel sei, was anzeigt, dass der literarische Wert auch anhand anderer Qualitäten als der Unterhaltung ermittelt werden muss.

Für sein eigenes Schaffen macht Fontane in einem Brief vom 23. Januar 1890 den Anspruch geltend, seine Stoffe 'nicht langweilig' behandeln zu wollen. ${ }^{202}$ Dies zeigt ein grundsätzliches Interesse daran, die Leserschaft zu unterhalten. Diese Einstellung bekommt umso mehr Bedeutung, als Fontane eine moralisch didaktische Funktion seiner Werke kategorisch ausschließt. Im Brief vom 10. Februar 1891 schreibt er: 'Soll die Kunst den Moralzustand erhalten oder bessern, so haben Sie recht, soll die Kunst einfach das Leben widerspiegeln, so habe ich recht. Ich wollte nur das letztre'.203 Fontane weist jegliches moralisches Engagement von sich. Bei der Widerspiegelung des Lebens unterscheidet Fontane in der 'Lyrischen und epischen Poesie' zwischen Wirklichem und Wahrem. Seine Ausführungen zu dieser Dichotomie bleiben jedoch vage. Da er das Wirkliche als 'das bloß Handgreifliche' im Gegensatz zum Wahren setzt, kann angenommen werden, dass es sich bei letzterem um eine poetisch aufgearbeitete Darstellung des Wirklichen handelt. ${ }^{204}$ Darüber hinaus dient Fontane die Auswahl des Sujets auch dazu, den Leser für ein Werk zu gewinnen. In der 'Lyrischen und epischen Poesie' äußert er: 'Der Realismus hält auch nichts von dem, was unserm Interesse völlig fremd geworden ist'. ${ }^{205}$ Mit 'uns' meint Fontane die gesamte deutsche Gesellschaft. Er selbst habe bei der Themenwahl, wie er in einem Brief vom 23. Januar 1890 anführt, vor allem eine Präferenz für die mittleren und kleinen Stoffe, die großen habe er immer gemieden. ${ }^{206}$ Dies spricht für eine liberale Einstellung gegenüber einer breiteren und bildungsfernen Gesellschaftsschicht, für die das eigene Werk lesbar sein

\footnotetext{
201 Fontane 1968-1971, Bd. 4, S. 106.

202 Fontane 1980, Bd. 2, S. 256.

203 Ebd., S. 276.

204 Vgl. Fontane 1962-1997, Abteilung 3, Bd. 1, S. 242.

205 Ebd.

206 Vgl. Fontane 1980, Bd. 2, S. 255.
} 
soll. Die textuelle Wirkungsabsicht, für die Fontane am intensivsten eintritt, ist das movere. Am 12. Februar 1895 äußert er hinsichtlich der Romanliteratur: 'Zu unserm Gefühl muss gesprochen werden'. ${ }^{207} \mathrm{Zu}$ Theater und Oper schreibt er am 1. Februar 1894: 'So beglücken zu können, enthusiastische Gefühle zu wecken, das ist doch das Schönste an der Kunst'. ${ }^{208}$ Fontane lehnt von den drei rhetorischen Wirkungsabsichten demnach das docere ab, tritt für das delectare ein und sieht das movere als privilegierte Funktion an.

Unterhaltung spielt auch bei Fontanes Einstellung zur Breitenwirkung eine Rolle. Am 5. Dezember 1890 schreibt er in einem Brief, es stehe schlecht um den Roman, da die Werke der jüngeren Generation dem Leser nicht genug Vergnügen bereiten, sodass die Jungautoren vom Publikum mit einer 'kolossalen Gleichgültigkeit gestraft' worden seien. ${ }^{209}$ Fontane sieht einerseits das delectare als Publikumserfolg begünstigende Kategorie an, andererseits sorgt sich Fontane um den Publikumserfolg. Ebenso etabliert er ein leichtes Textverständnis als den Publikumserfolg fördernde Qualität. In der 'Lyrischen und epischen Poesie' folgert er daraus, dass Scherenberg sich nicht darum kümmere, ob seine Leserschaft seine Ausführungen verstehe, dass Scherenberg 'im Munde künftiger Geschlechter aber [...] nicht leben wird, denn er mag alles sein, nur eines ist er sicherlich nicht - populär' ${ }^{210}$ Fontane rekurriert auf die prestigeträchtige Kategorie des Nachruhms. Durch die Verknüpfung von Publikumserfolg und Nachruhm wird erstere Kategorie als Grundvoraussetzung für letztere aufgewertet.

Fontane vertritt einen komplexen Erfolgsbegriff, der im Hinblick auf Lesbarkeit noch in Bezug auf finanziellen Erfolg auszuloten ist. Er erkennt einen zunehmend betrieblichen Charakter der Literatur:

[I]ch neige mehr und mehr der Ansicht zu, daß die recht haben, die den ganzen Kunstbetrieb als ein Semmelbacken ansehn, von dem man lebt wie andre Gewerbsleute. Alles ist unsicher und schwankend. Von den jeweiligen Kolossalerfolgen jammervoller Dümmlinge will ich noch gar nicht mal sprechen. ${ }^{211}$

\footnotetext{
207 Ebd., S. 354.

208 Ebd., S. 317.

209 Ebd., S. 272.

210 Fontane 1962-1997, Abteilung 3, Bd. 1, S. 251.

${ }^{211}$ Fontane 1980, Bd. 2, S. 260.
} 
Fontane diagnostiziert verschärfte Marktbedingungen und plädiert für die Notwendigkeit des Broterwerbs, gesteht jedoch vermeintlich dilettantenhaften Künstlern finanziellen Erfolg nicht zu. Finanziellen Erfolg wünscht er demnach nur begabten Kollegen. Darin spiegelt sich Fontanes zwiespältiges Verhältnis zum Verkaufserfolg. Er schreibt am 28. April 1890: 'Man muß von seiner Arbeit schlecht und gerecht leben können, mehr ist nicht nötig. ${ }^{212}$ Während Fontane große Erfolge ablehnt, heißt er finanziellen Erfolg gut, solange er einem Autor gesicherte Lebensverhältnisse verschafft. Eine besondere Abneigung hegt er gegen jene, die einzig und allein den Verkaufserfolg anstreben. Im Brief vom 22. Oktober 1889 möchte Fontane 'die festeste Überzeugung aussprechen, daß hinter [Gerhart Hauptmann] [...] mehr steckt als hinter der andern Blase, die alle bloß nach der "Tantieme" schielen'. ${ }^{213}$ Fontane setzt Qualität und finanzielle Aspekte in Bezug. Dem hochgeschätzten Hauptmann stellt er Autoren gegenüber, die ausschließlich aus ökonomischen Interessen schreiben, denen er im Vergleich Qualität abspricht. Fontanes Realismusbegriff jedoch versieht Lesbarkeit mit einer hohen ästhetischen Qualität, die er durch den Einfluss der Rhetorik legitimiert, der Klarheit, Anschaulichkeit und leichte Textverständlichkeit sowie Publikumsaffinität zum Desiderat werden lässt.

Die Debatte um die Lesbarkeit erhält zu Beginn des 20. Jahrhunderts durch die Ablehnung des Realismus im Expressionismus neue Nahrung. In Der Sturm, einer der führenden expressionistischen Kunst- und Literaturzeitschriften, erklärt der Autor Ludwig Rubiner in seinem Artikel 'Dichter der Unwirklichkeit. Anmerkungen zu den Büchern des Max Brod' (1910) die Epoche des Realismus für beendet:

\footnotetext{
Alle paar Jahrhunderte einmal sind die großen Entdeckungsreisen auf dieser Erdkugel zu Ende, und die Wirklichkeit ist abgesucht. Große und unterscheidende Dinge der Greifbarkeit und Sichtbarkeit sind gefunden und beschrieben, und es gilt, wahrzunehmen, daß von neuem die Jahre einer großen Epoche abgelaufen sind, die das Wirkliche zu Geist machen will. ${ }^{214}$
}

Rubiner wendet sich nicht energisch von einem realistischen Literaturbegriff ab. Vielmehr handelt es sich um eine nüchterne Feststellung, die dem Realismus Anerkennung zugesteht und lediglich deswegen von einem Ende der Epoche spricht, da

\footnotetext{
212 Ebd., S. 262.

213 Ebd., S. 243.

214 Rubiner 1910, S. 107.
} 
keine neuen Errungenschaften mehr zu erwarten sind. Ähnlich steht es auch um den Naturalismus, der laut Rubiner eine Variation von Techniken darstellt, 'die stets nur über dasselbe Stoffgebiet gespannt wurden, die Wirklichkeit'. ${ }^{215}$ Es wird zu klären sein, ob mit der Abkehr von einem realistischen Literaturbegriff auch das aufgebaute Lesbarkeitspotenzial verloren ging.

Der Schriftsteller Rudolf Kurz verabschiedet in seiner 'Programmatisches' betitelten Einführung in den Sturm (1910) Unterhaltung zugunsten eines gehobenen Qualitätsanspruchs:

\begin{abstract}
Aber wir haben es dem Publikum so leicht gemacht, uns mißzuverstehen, daß es ein erlaubter Scherz ist, $\mathrm{zu}$ dem Niveau einer behaglich freundwilligen Unterhaltung herabzusteigen [...]. [...] Es ist ein Irrtum. Wir wollen sie nicht unterhalten. Wir wollen ihnen ihr bequemes ernst-erhabenes Weltbild tückisch demolieren. ${ }^{216}$
\end{abstract}

Die Höhenmetaphorik des Herabsteigens siedelt Unterhaltung erneut auf einer niederen Qualitätsebene an, da sie dazu dient, den Lesern die Lektüre möglichst angenehm zu gestalten. Die Dichter der Zeitschrift besetzen eine erhabene Position, die sie nicht aufzugeben gedenken, um ihre Leser zu unterhalten. Vielmehr sollen in der Leser- und Gesellschaft vorhandene Ansichten dekonstruiert werden. Georg Kaiser intensiviert dies in seinem Aufsatz 'Vision und Figur' (1918) und proklamiert eine 'Erneuerung des Menschen' als ultimatives Ziel des Schreibens. ${ }^{217}$

Dies eröffnet ein Spannungsfeld zwischen den Positionen des Autors und der Leserschaft und der Einstellung der Autoren gegenüber der Breitenwirkung und den Wirkungsabsichten. In den Artikeln des Sturms wird die Position des Autors oftmals durch religiöse Termini und Begriffe aus der Genieästhetik erhöht. ${ }^{218}$ Alle derartigen Äußerungen haben gemein, dass die Autoren auf eine originale Schöpfung abzielen und daher die Bedürfnisse der Leser missachten. Der Autor Joseph August Lux beansprucht in seinem Beitrag 'Kunst und Ethik' (1910) eine solche 'Originalität, wie es nie zuvor geschehen und nie mehr ein zweites Mal vorkommen [kann]', zu erreichen und setzt dies dezidiert in Opposition zur Absicht, dem Publikum gefallen zu wollen. ${ }^{219}$ Dies

\footnotetext{
215 Ebd.

216 Kurtz 1910, S. 2.

217 'Vision und Figur' in Kaiser 1971-1972, Bd. 4, S. 547-549, hier: S. 549.

218 Z.B. in Lux 1910, S. 13 und 14; Kraus, 1910, S. 26; Onno-Eisenbart 1910, S. 200; Soyka 1910, S. 259.

219 Lux 1910, S. 14.
} 
deutet auf das Selbstverständnis einer Autoren-Avantgarde, die sich einer Literatur verschreibt, die indifferent gegenüber ihrem Publikum ist. ${ }^{220}$ Deutlich zeigt sich dies im Beitrag 'Der Leser' (1910) des Schriftstellers und Journalisten Otto Soyka, der es dem Leser zwar zugesteht, als Konsument von der Literatur bedient werden zu wollen, verpflichtet jedoch die Autoren, 'literarische Schätze' gegenüber einem Publikum zu schützen, das es gewohnt sei, die Literatur als einen 'Kramladen' anzusehen, in dem der Kunde Befehle erteile. ${ }^{221}$ Soyka führt einen merkantilen Aspekt von Literatur ein, wonach sich die Schreibenden für Qualität und gegen finanzielle Interessen bzw. die Bedürfnisse des Publikums einsetzen. Der Fokus liegt eindeutig auf der Position des Autors, der als Schöpfer in den Mittelpunkt rückt. Eine Anpassung an den Geschmack der Leserschaft wird strikt abgelehnt. Dies widerspricht z.B. der von Wieland vertretenen Ansicht, dass Qualität erst durch finanziellen Erfolg gewährleistet werden könne. Zudem scheint sich Gutzkows Ansicht, dass avantgardistische Texte nicht publikumskompatibel sind, zu bestätigen. Die Ablehnung von Lesbarkeit stellt demnach einen wichtigen Aspekt des expressionistischen Literaturverständnisses dar.

Dementsprechend wird Breitenwirkung zurückgewiesen, was hauptsächlich mit einem Qualitätsverlust begründet wird. Lux schreibt in 'Kunst und Ethik': 'Was der Masse frommt, ich meine auch die Masse der Gebildeten, ist ein Durchschnitt, der sich auf der mittleren Linie bewegt, eine Kunst, die zu Tode erklärt ist' ${ }^{222}$ Lux etabliert den literarischen Mittelweg als qualitativ minderwertig und als Verhängnis der Literatur, wobei er allerdings bestätigt, dass eine Literatur der Mitte der Breitenwirkung förderlich ist. Ebenso lehnt der Autor Carl Onno-Eisenbart in 'Literarische Ausländerei?' (1910) den kommerziellen Aspekt von Literatur ab: 'Künstlerisches Wirken ist selbstlos, und einer Kunst, die bewußt nach Brot geht, fehlt gewöhnlich die Größe jener Schöpfungen, die innerer Notwendigkeit ihre Entstehung verdanken'. ${ }^{223}$ Aus Onno-Eisenbarts Aussage spricht ein idealistischer Einfluss: der Künstler soll sich mit seiner Kunst nur um ihrer selbst willen beschäftigen. Pekuniäre Interessen sind demnach mit Qualität unvereinbar. Den Grund dafür liefert Lux, der konstatiert, dass ein Autor, um in der Breite zu wirken,

\footnotetext{
220 Dass es sich dabei um ein im Expressionismus weiterverbreitetes Selbstverständnis der Autoren handelt, zeigt Walter Fähnders. Er verweist auf die gesellschaftliche Isolation des Dichters und Ansätze zu einem schriftstellerischen 'Messianismus', vgl. Fähnders 1998, S. 154-157; Im Reallexikon führt Georg Jäger an, dass speziell die Autoren des Sturm sich mit der Avantgarde identifizierten und auch von Literaturhistorikern besonders mit der Avantgarde assoziiert werden, vgl. Jäger 1997, S. 185.

221 Vgl. Soyka 1910, S. 259.

222 Lux 1910, S. 13.

223 Onno-Eisenbart 1910.
} 
'der namenlosen Menge zu gefallen suchen [muss], die, so verschiedenartig an Bildung und Menschlichkeit, nur einen sehr oberflächlichen Berührungspunkt haben kann'.224 Durch die Oberflächenmetaphorik dekonstruiert Lux die Qualität einer Literatur, die dem Publikumsgeschmack entspricht und auf eine breite Leserschaft zielt, da die Diversität des Publikums keine tiefgreifende Vorgehensweise erlaubt.

Die Qualitätsfrage wird im Sturm zudem anhand einer leichten Textverständlichkeit diskutiert. Lux fährt in seinen Ausführungen zum Publikumserfolg fort: '[D]er Künstler habe es als sittliche Forderung zu betrachten, daß er der Menge gefalle, daß er den niedrigsten Schönheitsbegriff zur Richtschnur nehme, daß er nur das zur Darstellung bringe, was auch dem gewöhnlichsten Mann verständlich bleibt. ${ }^{225}$ Lux etabliert leichtes Textverständnis als weitere Grundvoraussetzung für Breitenwirkung. Die Konzeption von Texten, die einer breiten Masse zugänglich sind, wertet er umgehend höhenmetaphorisch ab. Lux rückt diesen Lesbarkeitsaspekt in den Kontext der Verkaufsabsicht und der Anbiederung an das Publikum, indem er solchen Autoren, die leicht verständliche Texte schreiben, die 'schamlose Verlogenheit, mit der sich jede Dirne auf der Straße verkauft', attestiert. ${ }^{226}$ Verständlichkeit klassifiziert er demnach generell als verwerfliche Verkaufsstrategie.

Jedoch finden sich auch im Expressionismus gegenläufige Auffassungen zu Publikumsfreundlichkeit und Breitenwirkung. In der Literaturzeitschrift Das neue Pathos (1913) etabliert Stefan Zweig jene beiden Kategorien als wichtige Programmpunkte. ${ }^{227}$ Seit das Schrifttum den mündlichen Vortrag als Rezeptionsort von Literatur abgelöst habe, argumentiert Zweig in seinem Plädoyer für die Rhetorik, hätten sich die Schriftsteller ästhetisch von ihrem Publikum entfernt. Besonders zu Beginn des 20. Jahrhunderts habe sich deswegen eine poetische Sprache entwickelt, die 'weltfremd' und daher 'dem größten Teil der Tätigen, den Wirklichen, dem Arbeiter und dem Werkmann fremd und wertlos geworden ist. ${ }^{228}$ Zweig tritt für eine am Alltag orientierte Sprache ein, die Verständlichkeit fördert und dem Publikum die Literatur näher bringt.

\footnotetext{
${ }^{224}$ Lux 1910, S. 14.

225 Ebd.

226 Ebd.

${ }^{227}$ Es muss darauf hingewiesen werden, dass sich Zweig überwiegend auf den mündlichen Vortrag bezieht. Daher kann nicht im engeren Sinne von Lesbarkeit gesprochen werden. Dennoch finden sich in Breitenwirkung und Begeisterung Kategorien, die für eine Auseinandersetzung mit der Lesbarkeitsdebatte nützlich sind.

${ }^{228}$ Zweig 1913, S. 3.
} 
Das Pathos fungiert als Begeisterungsmittel, um die Nähe zum Rezipienten durch Vergnügen und Gefühlsregung herzustellen.229

Im Expressionismus gab es demnach Strömungen, die für eine publikumswirksame Literatur eintraten und dabei von rhetorischen Einflüssen inspiriert wurden. Diese konkurrierten mit einem elitären Publikums- und Literaturbegriff, die gegen Lesbarkeit aber für ein avantgardistisches und komplexes Literaturprogramm standen.

Erich Kästner und die Vertreter der 'Neuen Sachlichkeit' hingegen setzten sich in Opposition zu den exklusiven Literaturprogrammen des Expressionismus und des Symbolismus und strebten eine leserfreundliche Literatur an. ${ }^{230}$ Kästner macht als Wirkungsabsicht vorwiegend, aber - wie z.B. seine unterhaltsamen Kinderbücher zeigen - nicht ausschließlich, einen Gebrauchswert mit gesellschaftskritischer Dimension für die Leser geltend.

Angesichts seines gattungs- und genreübergreifenden Werks beschränkt sich bei Kästner die Anwendung des Lesbarkeitsbegriffs nicht auf die Gattung der Prosa. Seine poetologischen Äußerungen beziehen sich auch auf die Lyrik. Die Basis für Kästners Autorpoetik liefert die Abkehr vom Expressionismus. ${ }^{231}$ In einem Beitrag für die Monatsschrift Das deutsche Buch (1928) kritisiert Kästner am Expressionismus, dass ihm 'das große Publikum [...] beziehungslos gegenüber[stand]'. ${ }^{232}$ Kästner sucht die Nähe zum Publikum und nimmt die Autoren in die Pflicht, ihre Texte lesbar zu gestalten. In seinem programmatischen Epigrammband Kurz und bündig (1950) schreibt Kästner in dem Epigramm 'Präzision':

Wer was zu sagen hat, hat keine Eile.

Er läßt sich Zeit und sagt's

in einer Zeile. ${ }^{233}$

\footnotetext{
229 Vgl. ebd., S. 3-5.

230 Es ist bekannt, dass sich Erich Kästner von dem Begriff der 'Neuen Sachlichkeit' distanzierte. Thomas von PlutoProndzinski zeigt jedoch, dass rein ästhetisch keine Zweifel an der Zugehörigkeit Kästners zur 'Neuen Sachlichkeit' bestehen könnten; vgl. Pluto-Prondzinski 2014.

231 Die Kritik bezog sich ebenso auf den Symbolismus, wenn auch nicht mit derselben Intensität und Prominenz; für die Fragestellung dieser Arbeit reicht es aus, Kästners Expressionismuskritik exemplarisch darzustellen.

232 Kästner 1928, S. 143.

233 'Kurz und bündig', in Kästner 1959, Bd. 1, S. 317-346, hier: S. 321.
} 
Kästner rekurriert auf die Lesbarkeit fördernden rhetorischen Qualitäten der brevitas und der claritas. Er betont durch die Verwendung der temporären Kategorien der 'Eile' und des 'Sich-Zeit-Lassens' die Intensität des Arbeitsaufwands, den eine möglichst kurze und präzise Schreibweise erfordert. Dadurch unterstreicht er die zur Umsetzung nötige schriftstellerische Kompetenz und etabliert eine auf das Wesentliche konzentrierte Ausdrucksweise als qualitativ hochwertig. ${ }^{234}$

In Kästners Autorschaftsentwurf und inhaltlicher Gestaltung zeigt sich eine Hinwendung zum Leser. Hinsichtlich der Autorschaftskonzeption schreibt er im Vorwort seiner Gedichtanthologie Die 13 Monate (1955): '[die Gedichte] schrieb [...] ein Großstädter für Großstädter'. ${ }^{235}$ Zwar schließt Kästner in diesem spezifischen Beispiel alle Nicht-Großstädter als Adressaten aus und zielt demnach nicht auf ein größtmögliches Publikum, jedoch etabliert er ein egalitäres Verhältnis zwischen Autor und Leser. ${ }^{236}$ Der Autor nimmt keine genialisch exponierte Stellung gegenüber seinem Publikum ein, sondern schreibt als Gleicher unter Gleichen, wodurch ein effektiveres Kommunikationspotenzial gegeben ist. Durch diese Konstellation kann von einer gemeinsamen sprachlichen wie thematischen Grundlage des Schreibenden und des Lesenden ausgegangen werden.

Dieses Heranrücken an den Leser zeigt sich auch an Kästners Rückgriff auf realistische Gestaltungsweisen. In Das Deutsche Buch nennt Kästner einen 'Trieb zur Wirklichkeit' als Basis für die Abkehr vom hochambitionierten Expressionismus und der Zuwendung zu einer Breitenliteratur. ${ }^{237}$ Im Vorwort zum Roman Emil und die Detektive (1929) diskutiert eine Autorfigur, die den Namen Kästner trägt, mit einem Oberkellner, der die breite Masse repräsentiert, die Kästner als höhere Autorität ansieht als Literaturwissenschaftler oder -kritiker, über die Möglichkeit, einen in der Südsee angesiedelten Abenteuerroman zu schreiben. Der Kellner überzeugt die Figur Kästner, davon abzulassen: 'Und so habe ich, eigentlich nur, weil der Oberkellner Nietenführ es so wollte, eine Geschichte über Dinge geschrieben, die wir, ihr und ich, längst

\footnotetext{
${ }^{234}$ Von Pluto-Prondzinski verweist zudem darauf, dass diese 'Präzisionsästhetik' (Sabina Becker) vor allem dazu diene, das Publikum für das neusachliche Literaturprogramm zu gewinnen: 'Die folglich klare und eher bescheidene Sprache ist ein Aspekt der Ästhetik der Neuen Sachlichkeit, der die Schwelle zu literarischen Texten für ein breites Publikum absenkt.' (Pluto-Prondzinski 2014, S. 232); der Terminus der 'Präzisionsästhetik' wird auch auf Prosa und speziell auf Romane angewendet, vgl. Becker S. 2000, S. 129-138.

235 'Die dreizehn Monate', in Kästner 1959, Bd. 1, S. 347-391, hier: S. 351.

${ }^{236}$ Es geht Kästner bei seinem Autorschaftsmodell vor allem um die Nähe zum Publikum. Angestrebt wird ein möglichst großer Realitäts- und Aktualitätsbezug. Dazu trägt auch die Wahl der Großstadt als typisches Sujet der 'Neuen Sachlichkeit' bei.

${ }^{237}$ Kästner 1928, S. 144.
} 
kennen. ${ }^{238}$ Das Alltägliche avanciert zum literarischen Stoff, der an die Lebenswirklichkeit der Leser anknüpft. Das Autor und Leser einschließende 'wir' führt zu einer auf gemeinsamer Erfahrung aufbauenden Komplizenschaft zwischen Kästner und seinem Publikum. Dass allerdings keine getreue Abbildung der Realität angestrebt wird, zeigt das Vorwort zu Pünktchen und Anton (1931):

Ob wirklich passiert oder nicht, das ist egal. Hauptsache, daß die Geschichte wahr ist! Wahr ist eine Geschichte dann, wenn sie genau so, wie sie berichtet wird, wirklich hätte passieren können. [...] Wenn ihr [die Kinder] das verstanden habt, habt ihr ein wichtiges Gesetz der Kunst begriffen. ${ }^{239}$

Wie Fontane zieht Kästner das Wahre dem Wirklichen vor. Eine Erzählung speist sich zwar aus der Wirklichkeit, ist aber nicht an sie gebunden. Wahrhaftigkeit kann also als philosophische Kategorie der Plausibilität des Erzählten begriffen werden und verweist auf das aristotelische Mimesisprinzip. Zudem versieht Kästner seine auf 'wahre' Geschichten ausgelegte Kinderliteratur mit dem Kunstprädikat, was der Kinder- und Jugendliteratur ihm zufolge zu seiner Zeit oft abgesprochen wurde. ${ }^{240}$ Zudem deutet der letzte Teilsatz darauf hin, dass Kästner eine Kunsterziehung des Rezipienten zum mündigen Leser betreibt, die Schillers Postulat der Ausprägung eines Publikumsgeschmacks gleicht.

Mit dem Realismus verfolgt Kästner zwei Ziele: Das erste erschließt sich aus einem weiteren Kurz und bündig-Epigramm. Kästner fordert seine Autorenkollegen auf: 'Die Zeit zu schildern, ist eure heilige Pflicht. / Erzählt die Taten! Beschreibt die Gesinnungen!'. ${ }^{241}$ Entgegen der Vorstellung von einem Autor, der als Großstädter für Großstädter schreibt, führt Kästner mit dem Adjektiv 'heilig' eine transzendente Funktion des Schreibens ein. Diese Funktion beinhaltet die Aufgabe einer nicht-

\footnotetext{
${ }^{238}$ Emil und die Detektive, in Kästner 1959, Bd. 6, S. 159-265, hier: S. 164.

239 Pünktchen und Anton, in ebd., S. 413-509, hier: S. 416; dies korrespondiert mit dem Begriff der 'Wahrscheinlichkeit' aus dem 9. Buch der Poetik des Aristoteles, vgl. Aristoteles 1994, 9, S. 29-33.

${ }^{240}$ Kästner beklagt diesen Misstand in seiner Rede zur Kundgebung für das Jugendbuch (1953). Die Kritik an der Jugendliteratur beziehe sich hauptsächlich auf die notwendige Vereinfachung der Darstellung. Daher sei es dazu gekommen, dass Schriftsteller, die nur Jugendbücher schrieben, nicht als Schriftsteller angesehen wurden, da nur jene Jugendbücher schrieben, die nicht schreiben könnten. Zum Symbol für dieses Missverständnis wird für Kästner das Adverb 'nur'. Zu oft lese man, es handle sich 'nur' um Jugendliteratur. Aufgrund dieses Stigmas schreckten viele fähige Autoren davor zurück, Jugendbücher zu schreiben. Kästner jedoch fordert seine Kollegen auf, sich gerade jenem Genre zuzuwenden, um einem Erziehungsauftrag für die Jugend gerecht $\mathrm{zu}$ werden; vgl. 'Jugend, Literatur und Jugendliteratur', in Kästner 1959. Bd. 5, S. 505-513.

241 'Die Wirklichkeit als Stoff', in Kästner 1959, Bd. 1, S. 340.
} 
didaktischen Dokumentation der Zeit, wodurch Kästner ironisch die künstlerische Selbstisolation des Elfenbeinturms unterläuft.

Das zweite Ziel stellt eine unmittelbare Funktion der Literatur im Leben ihrer Leser dar. In Kästners Prosaischer Zwischenbemerkung (1929) heißt es:

\begin{abstract}
Man hat für diese Art von Gedichten die Bezeichnung 'Gebrauchslyrik' erfunden, und die Erfindung beweist, wie selten in der jüngsten Vergangenheit wirkliche Lyrik war. Denn sonst wäre es jetzt überflüssig, auf ihre Gebrauchsfähigkeit wörtlich hinzudeuten. Verse, die von den Zeitgenossen nicht in irgendeiner Weise zu brauchen sind, sind Reimspielereien, nichts weiter. ${ }^{242}$
\end{abstract}

Kästner wendet sich gegen die Vorstellung des l'art pour l'art. Die Gebrauchsfähigkeit für den Leser sieht der Berufsschriftsteller Kästner als inhärente Qualität der Kunst. Dies ist ein Kunstverständnis, das die Position des Lesers im Vergleich zu der des Autors im Literaturbetrieb deutlich aufwertet. Die Literatur wird in den Dienst des Lesers gestellt, rückt in die unmittelbare Nähe des Lesers und muss für diesen verständlich gestaltet werden.

Die Analyse der Autorpoetik Kästners zeigt, dass auch in der Moderne lesbare und Breitenwirkung anstrebende literarische Formen nicht nur vertreten waren, sondern auch ausdrücklich gefordert wurden. Zieht man zudem einen Brief Thomas Manns an Hermann Hesse heran, stellt sich die Frage, ob die Moderne nicht einer rezeptionellen Überhöhung anheimgefallen ist, die eine genauere Reevaluierung der Epoche nötig macht. Thomas Mann - das im Literaturstreit der 1990er Jahre angeführte Paradebeispiel moderner Qualitätsliteratur - reflektiert über die Vielfalt der Kritiken zu seinem Roman Königliche Hoheit (1909) und folgert, dass es ihm, wie intendiert, gelungen sei, ein möglichst breites und diverses Publikum anzusprechen:

Nietzsche spricht einmal von Wagners 'wechselnder Optik': bald in Hinsicht auf die gröbsten Bedürfnisse, bald in Hinsicht auf die raffiniertesten. Dies ist der Einfluß, den ich meine. Und ich weiß nicht, ob ich je den Willen finden werde, mich seiner völlig zu entschlagen. Die Künstler, denen es nur um eine CoenakelWirkung zu thun ist, war ich stets geneigt, gering zu schätzen. Eine solche Wirkung würde mich nicht befriedigen. Mich verlangt auch nach den Dummen. ${ }^{243}$

${ }^{242}$ Aus 'Lärm im Spiegel' in ebd., S. 97-156, die 'Prosaische Zwischenbemerkung' findet sich ebd., S. 125f, hier: S. 126.

${ }^{243}$ Mann 2002-2011, Bd. 1, S. 448. Der letzte Satz ist in der zitierten Ausgabe kursiv. 
Die 'wechselnde Optik' gedenkt Mann dadurch zu erreichen, dass er in seinen Texten 'populäre' mit 'artistischen' Elementen mischt. ${ }^{244}$ Auf diese Weise erklärt er es zum Ziel, eine bildungsferne Leserschaft anzusprechen, lehnt es allerdings ab, populäre Elemente heranzuziehen, wenn sie einzig dem Zweck des 'bewusste[n] Liebäugeln[s] mit dem Publikum' dienen. ${ }^{245}$ Mit diesem Literaturbegriff nimmt Mann die postmoderne Strategie des doppelkodierten Vergnügens vorweg, die dazu eingesetzt wird, eine möglichst breite Leserschaft anzusprechen. ${ }^{246}$

Im Nationalsozialismus nahmen Breitenwirkung und Lesbarkeit in einem kompetitiven Wettstreit um die Vorherrschaft bei der literarischen Ausrichtung des NS-Apparates eine zentrale Stellung ein. Zwar verfolgten alle Beteiligten das Ziel, eine nationalsozialistische Literatur zu schaffen, jedoch konnte dies aufgrund der zahlreichen Einfluss nehmenden Institutionen nicht umgesetzt werden. ${ }^{247}$ Ein Fürsprecher von Lesbarkeit war Propagandaminister Joseph Goebbels, ${ }^{248}$ der in seiner Rede auf der Herbstveranstaltung des deutschen Schrifttums in Weimar (1942) für Unterhaltungsliteratur eintritt und ein umfassendes Lesbarkeitsprogramm entwirft:

ein leichtes, fesselndes Schrifttum, das keinen großen seelischen Aufwand erfordert, sondern unaufdringlich vom Alltag hinwegführt. Inhalt wie Sprache müssen der breiten Masse unserer Volksgenossen und unserer Soldaten ohne

\footnotetext{
244 Ebd.

245 Ebd.

246 Der Romanist Gerhard Regn erläutert: 'Das postmoderne Vergnügen ist doppelt kodiert. Es stell sich zum einen dar als das unmittelbare, exoterische Vergnügen an der erzählten Geschichte; und zum anderen präsentiert es sich als das esoterische Vergnügen am Spiel des Erzählens, und das heißt vor allem: am subtil-elitären Spiel der Intertextualität und des Jonglierens mit verschiedenen Gattungskonventionen.' (Regn 1992, S. 69). Das 'esoterische Vergnügen' fungiert als Qualitätsgarant. Im Anschluss an die Tradition des poeta doctus muss ein Autor gebildet sein, um das Spiel mit intertextuellen Einflüssen zu meistern. Das Einarbeiten von Texten zielt auf literarische kompetente Leser und vor allem Kritiker, die Freude aus der Entschlüsselung der Anspielungen ziehen. Um auch die breite Leserschaft zu unterhalten, müssen die postmodernen Texte über ihre Handlung funktionieren. Texte werden durch die Verarbeitung von literarischen Vorbildern mit Semantik aufgeladen und komplexer gestaltet, zugleich müssen diese so integriert werden, dass sie dem Leser keine Schwierigkeiten bei der Lektüre bereiten.

${ }^{247}$ In seiner 2010 erschienen Monographie zu Bestsellern in der Nazizeit Lesen unter Hitler spricht Christian Adam von einem 'Kompetenz-Wirrwar' ; siehe Adam 2010, S. 19.

248 Es muss berücksichtigt werden, dass Goebbels' Befürwortung von Unterhaltungsliteratur als pragmatisch und opportunistisch angesehen werden kann und zeitlichen Gegebenheiten unterworfen war. Adam führt an, dass unter dem Naziregime tendenziell ein Hang dazu herrschte, Bestsellern literarische Qualität abzusprechen. Goebbels Gewogenheit gegenüber unterhaltsamer Literatur gründete, so Adam, nicht zuletzt darin, dass sich mit ihr viel Geld verdienen ließ. Später hätte das Regime die Verkaufszahlen konformer Autoren sogar zu Werbezwecken eingesetzt. Unterhaltung wurde Adam zufolge als Spezifikum anglo-amerikanischer Literatur angesehen, von der man sich insbesondere nach Kriegseintritt der Vereinigten Staaten abzuwenden gedachte: Wurden englischsprachige Unterhaltungswerke zuvor noch in Ermangelung einer nazideutschen Unterhaltungsliteratur und dem Verlangen der Gesellschaft nach Unterhaltung geduldet, disqualifizierten sie Kritik und Goebbels' Propagandaministerium später als 'Pulp Fiction', was im Oktober $1940 \mathrm{zu}$ einem Verbot führte. Goebbels zog demnach eine qualitative Untergrenze, die das deutsche Volk vor amerikanischem Schund bewahren sollte und nutzte diese Maßnahme gleichsam zur Propaganda gegen das vermeintlich bildungsferne Amerika; vgl. ebd., v.a S. 48f, 54 und 205f.
} 
weiteres zugänglich sein; ein frischer und fortschreitender Fluß der Handlung, ohne langatmige Ausdeutungen und Betrachtungen soll den Leser fesseln und in den Bannkreis des Buches ziehen. Gerade diese Erzählungen sind es, die seit Kriegsbeginn wieder und wieder von der Wehrmacht und vom Volk in der Heimat begehrt werden. ${ }^{249}$

Goebbels begründet seine Sympathie für unterhaltende Lesestoffe damit, die Nachfrage des Publikums befriedigen zu wollen, und etabliert Breitenwirkung als explizites Ziel. Indem Goebbels den Eskapismus als literarische Funktion hervorhebt, betont er, dass Unterhaltungsformen während des Zweiten Weltkriegs vor allem dazu dienten, die Leser zu bannen und vom Kriegsalltag abzulenken. Dies soll möglichst unterschwellig vonstattengehen, was dadurch erreicht werden soll, dass dem Leser sowohl sprachlich als auch thematisch keine Hindernisse in den Weg gestellt werden. Besonders hebt Goebbels die Bedeutung der Handlung hervor. Im aristotelischen Sinne sollten sich die Autoren auf das Fortschreiten der Handlung konzentrieren und von ausgiebigen Kommentaren oder weiterführenden Gedankengängen absehen. Einen Qualitätsanspruch erhebt Goebbels nicht.

Goebbels instrumentalisiert Lesbarkeit zu dem propagandistischen Zweck, die deutsche Leserschaft von den Kriegsvorgängen abzulenken. Vielleicht liegt gerade darin die von Heinrich Böll in seinen Frankfurter Poetikvorlesungen (1964) konstatierte Abwesenheit einer Unterhaltungsliteratur im Nachkriegsdeutschland begründet. ${ }^{250}$ Die in der Nazizeit instrumentalisierte Unterhaltungsliteratur konnte sich nicht mit einer engagierten Nachkriegsliteratur, die sich literarisch und moralisch mit dem Dritten Reich auseinanderzusetzen gedachte, als kompatibel erweisen.

Thomas Mann macht es den Autoren in seiner Ansprache im Goethejahr 1949 zur Aufgabe, moralisch und politisch die Zukunft Deutschlands zu gestalten, wodurch er den zentralen Konflikt des Literaturstreits zwischen Ästhetik und Engagement vorwegnimmt, der die folgenden Jahrzehnte bestimmen sollte. Gerade in der Frage nach der zukünftigen geistigen und moralischen Ausrichtung Deutschlands nimmt Mann die Schriftsteller in die Pflicht, entschieden gegen eine Teilung des Landes einzutreten: 'Wer sollte die Einheit Deutschlands gewährleisten und darstellen, wenn nicht ein unabhängiger Schriftsteller, dessen wahre Heimat [...] die freie, von Besatzungen

\footnotetext{
249 Joseph Goebbels in ebd., S. 171.
}

250 Vgl. Böll 1977, S. 87. 
unberührte deutsche Sprache ist'. ${ }^{251}$ Mann plädiert für das Ideal eines sich durch politisch-ideologische Unabhängigkeit definierenden freien Schriftstellertums, das er auf den Aktivismus zur Bewahrung der deutschen Einheit verpflichtet. Mann exponiert die Bedeutung der Sprache als kulturelle Heimat und als Vehikel, das auf Goethe und die Weimarer Klassik verweist, in der das kleinstaatlich geteilte Deutschland ebenfalls keine Einheit darstellte.

Mann erkennt weitere Funktionen der deutschen Nachkriegsliteratur, deren erste ein positiv besetzter Eskapismus ist:

Wenn nicht die Zuflucht der Phantasie wäre, wenn sie nicht wären, die [...] zu steigerndem Weitermachen verführenden Spiele und Unterhaltungen des Fabulierens, [...] der Kunst - ich wüßte nicht, wie zu leben, von Rat und guter Lehre für andere ganz zu schweigen. ${ }^{252}$

Die Metapher der Zufluchtsstätte zielt auf die Bedeutung von Literatur für die eigene Person und den Schreibprozess ab - nicht auf die Immersion des Lesers. Die Flucht in die Fantasie wird zur Grundbedingung der Existenz. Beim Schreiben, dem auch eine Unterhaltungsfunktion zukommt, sollen immer neue künstlerische Möglichkeiten ausgelotet werden. Darüber hinaus deutet sich eine didaktische Funktion für den Leser an. Sowohl bei der Ästhetik als auch bei der Didaktik teilt Mann der Doppelkodierung des Attributes 'gut' eine exponierte Stellung zu:

Es $[\ldots]$ hat mit der Kunst vielleicht eine glücklichere, hilfreichere, lebensdienlichere Bewandtnis als mit allem Ratwissen, Glauben und Lehren. Der dankbare Laie und Genießer des Kunstwerks braucht zu dessen Lob und Preis das Wort 'schön'. Aber der Künstler, der Mann vom Bau, sagt gar nicht 'schön', er sagt: 'gut'. Er bevorzugt dies Wort, weil das fachlich Lebenswerte, das technisch Gekonnte sich besser und nüchterner darin ausdrückt. [...] Tatsächlich schwebt alle Kunst in der Doppeldeutigkeit dieses Wortes 'gut', in dem das Ästhetische und Moralische sich treffen, vermischen, ununterscheidbar werden, dessen Sinn übers bloß Ästhetische hinausreicht ins überhaupt Zustimmungswürdige und hinauf bis zur höchsten, gebietenden Idee der Vollendung. ${ }^{253}$

Dem Kontext entsprechend rekurriert Mann auf die ersten Verse aus Goethes 'Das Göttliche' ('Edel sei der Mensch / Hilfreich und gut!') und auf das Ziel der

\footnotetext{
251 Mann 1949, S. 10.

252 Ebd., S. 11.

253 Ebd.
} 
künstlerischen Vollendung. ${ }^{254}$ Die Vollendung besteht für Mann in der Symbiose aus ästhetischer und moralischer Qualität, die beide gleichermaßen das Streben zur Vollendung bedingen. Durch diese Kombination soll ein Bild geschaffen werden, 'in das man vieles hinein, aus dem man vieles herauslesen kann, und das doch nicht verwirrt, sondern durch Anschauung befriedigt und den Sinn für das Gute belebt'. ${ }^{255}$ Demnach sollen Texte komplex gestaltet werden, um dem Leser vielfältige Interpretationsansätze und -möglichkeiten zu erschließen, ohne ihn dabei durch deren Vielzahl zu überfordern. Mann strebt ein einheitliches Bild an, in dessen Kern die Fusion aus Ästhetik und Moral steht. Er plädiert demnach für das Zusammenspiel zweier Kategorien, die später von beiden Lagern des Literaturstreits als nicht zu vereinbarende Antipoden der deutschen Nachkriegsliteratur dargestellt wurden.

In der Bundesrepublik stellten die frühen Nachkriegsjahre die Literatur vor schwierige Bedingungen: Die Besatzungsmächte verschärften im Zuge der Demokratisierung und Re-education Deutschlands die Lizenzbedingungen, die Papierknappheit und die alliierte Papierzuteilung führten zu einer Privilegierung von Zeitungen und literarischen Kurzformen.

In den westdeutschen Besatzungszonen wurden früh Stimmen nach einem Neuanfang laut. Wolfgang Borchert äußert in seinem Essay 'Das ist unser Manifest' (1947) spezifische Vorstellungen über die junge deutsche Literatur, wobei er wie Mann das 'Gute' als moralisches Ziel des Schreibens anführt. Borchert definiert dies als möglichst realistische Darstellung der sozialen und gesellschaftlichen Umstände der Nachkriegszeit. ${ }^{256}$ Zur literarischen Darstellung der Zeit vertritt Borchert eine Reduktion der Sprache auf ihre elementare Bedeutung, die sich teilweise über sprachliche Normen hinwegsetzt und jene bricht:

Wir brauchen keine Dichter mit guter Grammatik. [...] Wir brauchen die mit dem heißen heiser geschluchzten Gefühl. Die zu Baum Baum und zu Weib Weib sagen und ja sagen und nein sagen: laut und deutlich und dreifach und ohne Konjunktiv. ${ }^{257}$

\footnotetext{
254 Vgl. Goethe 1985-1999, Abteilung 1, Bd. 2, S. 303 f.

255 Mann 1949, S. 12.

256 Vgl. 'Das ist unser Manifest', in Borchert 1949, S. 308-315, hier: S. 313.

257 Ebd., S. 310.
} 
Borchert verzichtet zu Gunsten einer emotionalen Erzählform auf Sprachrichtigkeit. Das Vokabular soll auf gängige einfache Bezeichnungen reduziert werden, poetische Umschreibungen lehnt Borchert ab. Darüber hinaus fordert er von Schriftstellern, energisch Stellung zu beziehen, und diese bedingungslos zu vertreten. Der Bruch mit grammatikalischen Regeln widerspricht dem Lesbarkeitsbegriff, wohingegen die sprachliche Reduktion auf einfaches und eindeutiges Vokabular als der Lesbarkeit förderlich erscheint.

Wolfgang Weyrauch vertritt ebenfalls ein Programm der poetischen Reduktion. Im Nachwort zu seiner Kurzgeschichtenanthologie Tausend Gramm (1949) entwickelt er einen Literaturbegriff, der 'um den Preis der Poesie' eine 'Bestandsaufnahme' anstrebt, was auf einen niedrigeren Qualitätsanspruch hindeutet. ${ }^{258}$ In seiner Bestandsaufnahme wählt Weyrauch für die Prosa die Metapher eines 'verschlungenen und finsteren Dickicht[s]'. 259 Dementsprechend fordert Weyrauch 'Kahlschläge' des literarischen Dickichts, einen Neuanfang in 'Sprache, Substanz und Konzeption'. ${ }^{260}$ Unter dem Vorsatz der Vereinfachung soll, so Weyrauch, das geschrieben werden, 'was ist'. ${ }^{261}$ Die Abbildung der Zeitumstände hat zwei Funktionen: erstens die eines engagierten Literaturprogramms, das zwar unpolitisch sein, jedoch auf das Leben der Menschen unterstützend einwirken solle, und, zweitens, den 'Zweck einer guten und schönen Lektüre'. ${ }^{262}$ Der vereinfachende Kahlschlag von Form und Sprache bringt demnach neben einer didaktischen Funktion eine Vereinfachung der Lektüre mit sich. Dies drückt sich auch in Weyrauchs Publikumskonzeption aus: 'zwei Literaturen [sollte es] geben: eine Literatur für jedermann [...] und eine Literatur des Versuchs [...], welche die kommende Literatur für jedermann vorbereitet'. ${ }^{263}$ Selbst eine experimentelle Literatur ist demnach darauf ausgerichtet, einmal einer breiten Leserschaft zugänglich zu sein.

Etwa zeitgleich formierte sich heftiger Widerstand gegen populäre Formen der Literatur und Kunst. Die Philosophen Max Horkheimer und Theodor Adorno nahmen mit Dialektik der Aufklärung. Philosophische Fragmente (1944) gewichtigen Einfluss auf die Bewertung von fiktionalen Werken, die nach finanziellem Erfolg und

\footnotetext{
258 Weyrauch 1949, S. 217.

259 Ebd., S. 210.

260 Ebd., S. 214.

261 Ebd., S. 217.

262 Ebd., S. 210.

263 Ebd., S. 209.
} 
Breitenwirkung streben. ${ }^{264}$ Ein ganzes Kapitel widmen Horkheimer und Adorno dem Begriff einer 'Kulturindustrie', mit dem sie eine auf die Massen ausgelegte Kultur verbinden, die hauptsächlich auf Vergnügen und Unterhaltung zielt. Sie beziehen sich zwar überwiegend auf Film, Fernsehen und Radio, jedoch kritisieren sie ausdrücklich literarische Werke, die ihrer Meinung nach auf Strategien der 'Kulturindustrie' rekurrieren. ${ }^{265}$

Horkheimer und Adorno setzen den Begriff der 'Kulturindustrie' in ein antipodisches Verhältnis zu einer 'avancierten Kunst': Sie grenzen eine auf Profit ausgerichtete Massenindustrie strikt von einer idealistisch geprägten nichtkommerziellen Kunst ab, der sie durch das Attribut 'avanciert' eine höhere Qualität zusprechen. ${ }^{266}$ Dabei sehen die Autoren die Marktentwicklungen als Ursache für einen Qualitätsverlust in der Literatur. ${ }^{267}$ Sie loben das Mäzenatentum des 19. Jahrhunderts, das es anständigen Verlegern erlaubt hätte, Autoren zu publizieren, die nicht mehr einbrachten als die Achtung des Kenners; erst das Verdikt von Angebot und Nachfrage hätte Autoren mit hohem ästhetischem Anspruch und Können dazu gezwungen, sich den Marktgegebenheiten unterzuordnen und entsprechend den Anspruch aufzugeben.

Zudem machen Adorno und Horkheimer den Qualitätsverlust an einer Mischform fest, die sich aus Elementen der 'Kulturindustrie' und der 'avancierten Kunst' speist. Dabei bewerten sie nicht nur die kulturelle Spaltung zwischen den Polen als negativ, sondern erkennen das Bemühen um eine Mischform nicht als valide Lösung der Problemstellung an: 'Der Gegensatz läßt am wenigsten sich versöhnen, indem man die leichte in die ernste aufnimmt oder umgekehrt'. ${ }^{268}$ 'Ernst' evoziert den Begriff der EKultur, der in der Terminologie Horkheimers und Adornos als 'avancierte Kunst' auftritt. Der 'leichte' Gegenpol ist wie jener der U-Kultur von Unterhaltung geprägt: Vergnügen sei 'der Gegensatz zur Kunst', die 'Kulturindustrie' sei als 'Kathedrale des gehobenen Vergnügens [verderbt]' ${ }^{269}$ Horkheimer und Adorno folgern: 'Die Fusion von Kultur und Unterhaltung vollzieht sich [...] als Depravation der Kunst. ${ }^{270}$ Unterhaltung führt demnach zwangsläufig zu einer qualitativen Abwertung, was die Autoren nicht

\footnotetext{
264 Zur Horkheimer, Adorno und der 'Frankfurter Schule' siehe Wiggershaus 1987.

265 Siehe z.B. die Besprechung von Hans Falladas Kleiner Mann - was nun? (1932) und Alfred Döblins Berlin Alexanderplatz (1929) in Horkheimer/Adorno 2000, S. 161f.

266 Vgl. ebd., S. 141.

$267 \mathrm{Vgl}$ ebd.

268 Ebd., S. 144.

269 Ebd., S. 150 und 151.

270 Ebd., S. 152.
} 
zuletzt auf eine Affinität von Unterhaltung und Markterfolg zurückführen. ${ }^{271}$ Sie vertreten somit einen elitären Kulturbegriff, der Unterhaltung als Wirkungsabsicht ausschließt und Breitenwirkung vor allem in der Form finanziellen Erfolgs stigmatisiert. Die Spannung zwischen den Polen der 'avancierten Kunst' und der 'Kulturindustrie' in einer mittleren Mischform aufzulösen, verurteilen sie als 'Heroisierung der Durchschnittlichkeit'. ${ }^{272}$

Horkheimer und Adorno sprechen ein Verdikt gegen unterhaltende und Breitenwirkung suchende Fiktion aus, dessen Auswirkungen auf die Bewertung von mittleren Literaturformen noch bis in den Literaturstreit und darüber hinaus nachklingen:

\begin{abstract}
Alle Massenkultur unterm Monopol ist identisch, und ihr Skelett, das von jenem fabrizierte begriffliche Gerippe, beginnt sich abzuzeichnen. [...] Lichtspiele und Rundfunk brauchen sich nicht mehr als Kunst auszugeben. Die Wahrheit, daß sie nichts sind als Geschäft, verwenden sie als Ideologie, die den Schund legitimieren soll, den sie vorsätzlich herstellen. Sie nennen sich selbst Industrien, und die publizierten Einkommensziffern ihrer Generaldirektoren schlagen den Zweifel an der gesellschaftlichen Notwendigkeit der Fertigprodukte nieder. ${ }^{273}$
\end{abstract}

Die Kritik richtet sich gegen eine vermeintlich schematische Gestaltung von breitenwirksamen Werken. Mit ihrer Metaphorik suggerieren Adorno und Horkheimer, dass es der Massenkultur an Substanz und vornehmlich an Kunstcharakter fehlt. Daher sehen die Werke der Massenkultur von einem künstlerischen Anspruch ab und definieren sich ausschließlich über finanziellen Erfolg, welcher der Selbstlegitimierung dient. Um dies $\mathrm{zu}$ zeigen, ziehen Horkheimer und Adorno die Metapher des 'Fertigprodukts' heran, das seine Existenz aufgrund seiner Verkaufszahlen legitimiert, immer den gleichen Standard garantiert, aber eine minderwertige Form der Verarbeitung darstellt.

Heinrich Böll wendet sich in seinem Aufsatz Bekenntnis zur Trümmerliteratur (1952) gegen die Stigmatisierung von leserfreundlichen Vereinfachungstendenzen in der frühen Nachkriegsliteratur: 'Die ersten schriftstellerischen Versuche unserer Generation nach 1945 hat man als Trümmerliteratur bezeichnet, man hat sie damit abzutun versucht. Wir

\footnotetext{
271 Vgl. ebd., S. 153.

272 Edb., S. 165.

273 Ebd., S. 128f.
} 
haben uns gegen diese Bezeichnung nicht gewehrt, weil sie zu Recht bestand. ${ }^{274}$ Böll macht für die Berechtigung des Terminus überwiegend thematische Gründe geltend, aus denen er eine Präferenz für eine realistische Darstellung der Nachkriegszeit und eine Abneigung gegenüber Beschönigungen ableitet: 'Die Zeitgenossen in die Idylle zu entführen, würde uns allzu grausam erscheinen, das Erwachen daraus wäre schrecklich, oder sollen wir wirklich Blindekuh miteinander spielen'?275 Böll wendet sich gegen eine eskapistische Beschönigung der Nachkriegsrealität und fordert seine Autorenkollegen auf, zur Vergangenheitsbewältigung die Realität der Gegenwart darzustellen.

Böll entwirft einen Realismusbegriff, der sich durch eine genaue Beobachtungsgabe und einen präzise eingesetzten Humor auszeichnen sollte. Humor kann als Form der Unterhaltung gelten. Dem gegenüber positioniert Böll 'BlindekuhSchriftsteller', deren Auge nach innen gewandt sei, um sich eine eigene Welt zu schaffen. Er betont die 'Unlesbarkeit' dieser Werke und nennt Hitlers Mein Kampf (1925-1926) als Bespiel, das nur wegen seiner 'Unlesbarkeit' davor bewahrt worden sei, verboten $\mathrm{zu}$ werden, und sich dennoch millionenfach verkauft habe. ${ }^{276}$ Böll sieht Lesbarkeit demnach als Voraussetzung für den Verkaufserfolg an. ${ }^{277}$

Böll stellt die Trümmerliteratur in die Nachfolge Homers, der mit der Ilias und der Odyssee ebenfalls Kriegs-, Trümmer- und Heimkehrerliteratur geschrieben habe; daher 'haben [wir, die Trümmerliteraten,] keinen Grund, uns dieser Bezeichnung zu schämen.'278 Böll setzt die Werke der Trümmerliteratur thematisch mit dem prestigeträchtigen Vorgänger in Verbindung und versucht dadurch unter den Autoren seiner Zeit ein Selbstbewusstsein zu kreieren, für die Trümmerliteratur einzustehen.

In seinen Frankfurter Poetikvorlesungen (gehalten 1964), in denen er eine ‘Ästhetik des Humanen' entwirft, sieht Böll wie Mann in seiner Rede zum Goethejahr Ästhetik und Moral als nicht trennbar an:

\footnotetext{
274 'Bekenntnis zur Trümmerliteratur', in Böll 1998-, Bd. 6, S. 58-62, hier: S. 58.

275 Ebd., S. 59.

276 Vgl. ebd., S. 61.

277 Zudem eröffnet er die Dimension der aus einer anti-realistischen Einstellung resultierenden Unlesbarkeit als Schutz vor Zensur. Die Validität des Beispiels Mein Kampf kann angezweifelt werden. Zunächst hätte des Werk nur im Zeitraum vom Erscheinen der beiden Bände (1925 bzw. 1926) bis zur Machtergreifung 1933 durch die Weimarer Gesetzgebung zensiert werden können, verkaufte sich jedoch bei weitem nicht so oft wie in den Jahren des Nationalsozialismus, in denen Mein Kampf zwar millionenfach veräußert (bzw. u.a. Ehepaaren aufgezwungen), aber kaum gelesen wurde.Vgl. Adam 2010, S. 115-118.
}

278 Ebd., S. 62. 
Moral und Ästhetik erweisen sich als kongruent, untrennbar auch, ganz gleich, wie trotzig oder gelassen, wie milde oder wütend, mit welchem Stil, aus welcher Optik ein Autor sich an die Beschreibung oder bloße Schilderung des Humanen begeben mag. 279

Auf Seiten der Moral sowie auf Seiten der Ästhetik lässt Böll unterschiedliche Gestaltungsformen zu, solange die Einheit von Moral und Ästhetik gegeben ist. Er etabliert diese Einheit als Notwendigkeit, um neue Formen der deutschen Sprache und Literatur ausbilden und sie von den Relikten der Nazizeit zu befreien:

Wenn Humanität wieder entstehen soll, ist eine mühselige Kleinarbeit zu verrichten, langweilig, lästig, viel Geduld erfordernd - in den Lesebüchern beginnend; im Kindergarten. Das ist es, was Ihnen bevorsteht - eine Ästhetik des Humanen zu bilden, Formen und Stile zu entwickeln, die der Moral des Zustands entsprechen. Vorsicht vor Worten, vor Feiern, an denen, musikalisch verbrämt, düsteres Pathos sich böse artikuliert. ${ }^{280}$

Böll fordert den Anstoß eines aktiven Humanisierungsprozesses von Sprache und Literatur, der ihm zufolge in Texten für Leseanfänger bzw. sogar früher einsetzen soll. Dies erinnert an Schillers und Kästners Desiderat der ästhetischen Bildung des Lesers. Geprägt von der 'Moral des Zustands' - der Auseinandersetzung mit dem nationalsozialistischen Erbe und der Zukunftsgestaltung - sollen eingedenk der von ihm postulierten Untrennbarkeit von Moral und Ästhetik neue ästhetische Charakteristika ausgebildet werden, mit denen eine ‘Ästhetik des Humanen’ begründet werden kann. Um diese Ästhetik durchzusetzen, müsste die deutsche Sprache sich jedoch erst von einem speziell im Dritten Reich ausgeprägten Pathos befreien. Entsprechend findet diese Ausprägung des movere, wie sie etwa im Expressionismus vertreten wurde, keinen Eingang in Bölls Poetologie.

Böll beschäftigt das Fehlen einer deutschen Unterhaltungsliteratur, wobei er besonders die von ihm als Subkategorien klassifizierten Genres des Kinder- und Jugendbuchs sowie des Kriminalromans herausstellt. Er macht geltend, dass der Literaturbetrieb $\mathrm{zu}$ seiner Zeit die Unterhaltungsliteratur in ein oppositionelles Verhältnis zu einer 'wahren' Literatur setzt:

\footnotetext{
279 Böll 1977, S. 84.
}

280 Ebd., S. 87. 
Ich habe den Unterschied zwischen der wahren und der Unterhaltungsliteratur nie wahrgenommen, d.h., ich habe mich frivolerweise von wahrer Literatur unterhalten lassen oder, was negativ deutlicher definiert werden kann: ich habe mich nur selten bei wahrer Literatur gelangweilt. ${ }^{281}$

Böll geht im Literaturbetrieb von einer Dichotomie aus, in der das Attribut 'wahr' für eine exkludierende Literaturauffassung steht, bei der nur ein bestimmter Literaturbegriff als Literatur bezeichnet werden kann. Das 'Wahre' erfüllt hier eine gänzlich andere Funktion als die aus der Mimesistradition bekannte der Plausibilität und steht für eine 'echte' Literatur, die Unterhaltungsliteratur vom literarischen Status ausschließt. Böll kritisiert und setzt die vom Literaturbetrieb angeführte Divergenz zwischen den Kategorien der 'wahren' und der Unterhaltungsliteratur außer Kraft, indem er Unterhaltung zur inhärenten Qualität der Kategorie einer 'wahren' Literatur erhebt.

Neben den Gegenpolen von 'wahrer' und unterhaltender Literatur attackiert Böll zudem den Unterschied zwischen engagierter und nicht engagierter Literatur:

ich [habe] den Unterschied zwischen engagierter und nicht engagierter Literatur [nie] wahr-, schon gar nicht ernst genommen. Ich wiederhole: es findet Betrug statt, wenn ein Autor seiner (jeweils für gut befundenen) Gesinnung wegen gelobt wird, ihm die Form verziehen oder diese nicht gewürdigt wird. [...] Eine Regel sollte gelten: je engagierter sich ein Autor glaubt, desto besser sollte er schreiben. Ich sehe jedenfalls nicht ein, daß ich mich irgendeiner Gesinnung wegen langweilen sollte..$^{282}$

Böll setzt im Literaturbetrieb das Fehlen eines ethisch-ästhetischen Konzepts und damit eine Spaltung zwischen Moral und Ästhetik voraus, die auf den Literaturstreit vorausdeutet. Wie Mann verwendet Böll 'gut' als Bewertungskategorie, jedoch kritisiert er Tendenzen des Literaturbetriebs, Moral und Ästhetik getrennt zu bewerten bzw. die moralischen Aspekte zu privilegieren. Dabei verwendet Böll den Terminus der 'Gesinnung', der später im Literaturstreit durch den Neologismus der 'Gesinnungsästhetik' zu Prominenz gelangen sollte. Bölls Kritik richtet sich an die Rezensenten und nicht an die Autoren, was daraus ersichtlich wird, dass er sich ebenso darüber echauffiert, dass ästhetische Qualität, wenn vorhanden, nicht gewürdigt wird. Er etabliert Unterhaltung als unmittelbare Folge einer ansprechenden Gestaltung, die er einer minderwertig präsentierten Gesinnung entgegensetzt, und legitimiert durch diese Verknüpfung sein Unterhaltungsdesiderat. 
Ebenso wie mit Unterhaltung sympathisiert Böll mit einer intellektuellen Kreisen fernstehenden Leserschaft und bekennt sich dazu, seine Literatur an ihren Bedürfnissen auszurichten: 'Man hat mich mit einiger Herablassung oft einen Autor der kleinen Leute genannt: peinlicherweise empfinde ich solche Einschränkungen immer als Schmeichelei.' ${ }^{283}$ Böll widersetzt sich einem Stigma, das der Literaturbetrieb an ihn herantrug. In der Terminologie Horkheimers und Adornos ist der Grund der Stigmatisierung von Bölls Gewogenheit gegenüber einer massenfreundlichen Literatur anstatt einer Präferenz für 'avancierte Kunst'. Böll identifiziert sich damit, Literatur für die 'kleinen' Leuten zugänglich zu gestalten. Durch den Begriff der Peinlichkeit unterläuft Böll subversiv die Erwartungshaltung des herablassenden Kritikers und stellt die Konvention der Privilegierung ästhetisch komplexer Werke in Frage.

In der DDR zeichnete sich im Programm des 'Sozialistischen Realismus' die Instrumentalisierung von Lesbarkeit zur politischen Erziehung des Volkes ab. Johannes R. Becher, Autor und Kulturminister der DDR, integriert in seinem poetologischen Sammelband Über Literatur und Kunst (1962) die Literatur in eine 'Massenkulturbewegung', die das Ziel verfolgt, die DDR zur 'Wiedergeburtsstätte einer neuen großen deutschen humanistischen Kultur' werden zu lassen. ${ }^{284}$ Becher vertritt eine äußerst spezifische Auffassung des Humanismus, den er mit einer politischen Aufgabe versieht und daher ins Zentrum einer auf Breitenwirkung ausgelegten Literatur stellt. Um größtmöglichen Einfluss zu nehmen, entwirft Becher ein egalitäres Zusammenspiel von Kunst, Wissenschaft und Politik: 'Die Aufgabe der Kunst muß es sein, sich auf die Höhe des Politischen zu erheben und einer seiner organischen Teile zu werden, wobei wir unter dem Begriff des Politischen die öffentlich wirksame Form der Menschenerziehung verstehen'. ${ }^{285}$ Becher strebt Breitenwirkung an, um Literatur und Kunst als politisches Instrument wirksam zu machen, wobei er der Politik durch die Höhenmetaphorik mehr Bedeutung als der Literatur zugesteht, die der Politik durch eine didaktische Funktion zu dienen hat. Zwar lehnt Becher Unterhaltung nicht als Wirkungsabsicht ab, jedoch soll sich ein Schriftsteller nicht mit der Rolle des 'Unterhalters' begnügen, sondern sich in die Politik einmischen. ${ }^{286}$ Auch das movere

\footnotetext{
283 Ebd., S. 16.

${ }^{284}$ Becher 1962, S. 10.

285 Ebd., S. 61.

286 Vgl. ebd., S. 67.
} 
spielt eine Rolle, da Bechers Literaturbegriff auf Gefühle und Emotionen zielen soll, um die sozialistische Gesinnung der Leser zu fördern: Literatur habe die Macht, 'den Menschen bis ins tiefste Innerste hinein [...] zu erschüttern und umzugestalten.'287 Becher rekurriert auf die expressionistische Tradition und ordnet die Gefühlserschütterung ebenfalls der didaktischen Funktion unter, um die Idee des Sozialismus in den Lesern zu implementieren.

Der 'Sozialistische Realismus' fungiert laut Becher als Mittel, um der 'Masse der Unterdrückten, den armen Bauern, den Heimarbeitern, dem Proletariat' zu dienen. ${ }^{288}$ Dieser Dienst bestehe insbesondere darin, inklusive Werke zu konzipieren, die von bildungsfernen Gesellschaftsteilen ohne größere Probleme gelesen werden können: 'Wir werden nicht umhinkönnen, uns auch mit der Herausgabe von Schriften zu befassen, welche diejenigen Menschen ansprechen, die bisher nicht die Gelegenheit gehabt haben, sich künstlerisch oder wissenschaftlich zu bilden.' ${ }^{289}$ Becher führt die Furcht des Bildungsbürgertums an, dass dieses Programm zu einer 'Niveausenkung' führen werde. ${ }^{290}$ Er selbst setzt sich vehement gegen diese negative Einstellung ein: 'Es wäre grundfalsch, an alles und jedes die höchste Anforderung $\mathrm{zu}$ stellen und nur Gipfelleistungen gelten zu lassen. Wir brauchen auch anständiges Mittelmaß besten Durchschnitts.' ${ }^{291}$ Becher zieht die Höhenmetapher der 'Gipfelleistung' zur qualitativen Einordnung heran. Er erkennt die Existenz jener qualitativ hochwertigsten Werke an, wertet jedoch gleichzeitig einen mittleren Literaturbegriff auf, was vor allem auf die Bildungsfunktion zurückzuführen ist. Die literarische Mitte wird nicht als qualitativ ebenbürtig etabliert, gelangt aber aufgrund ihres praktischen Nutzens zu Akzeptanz und Wert. Dies zeigt auch die folgende kulinarische Metapher: 'Kunst ist [...] kein Luxus mehr und keine Delikatesse für Feinschmecker, Kunst ist ein Lebensmittel, das entscheidend mitbestimmt den geistigen und moralischen Ernährungszustand eines Volkes. ${ }^{292}$ Die hochwertige Kunst beschreibt Becher mit dem Bild des Gourmets. Er löst die Kunst von einem hohen Qualitätsanspruch und stellt sie durch die Analogie zur

\footnotetext{
287 Ebd., S. 33.

288 Vgl. ebd., S. 448.

289 Ebd., S. 7; an anderer Stelle heißt es: 'Die Zeiten sind endgültig vorbei, daß die Kultur nur das Privileg einer verhältnismäßig kleinen Schicht war. Es kann und wird keine neue deutsche Kultur geben, die nicht den Dienst am Volk als ihre höchste Sendung betrachtet und die nicht ihre wesentlichste Verpflichtung darin sieht, die Bildungs- und Wissensschätze allen zugänglich zu machen', ebd., S. 15.

290 Vgl. ebd., S. $5 f$.

291 Ebd., S. 7.

292 Ebd., S. 3.
} 
'Ernährung' in einen noch bedeutenderen, da existenziell wesentlichen, Kontext, in dem ihre Bedeutung der Funktion gegenüber der Gesellschaft entstammt. ${ }^{293}$

Um Lesbarkeit zu garantieren, führt Becher verschiedene Qualitäten an, die er dem Realismusgebot unterwirft: die sprachliche und thematische Gestaltung sowie die Figurenzeichnung. ${ }^{294}$ 'Zugänglichkeit', die für Becher die literarische Umsetzung des Demokratiegedankens darstellt, avanciert zum Schlagwort seines Realismusbegriffs. ${ }^{295}$ Becher etabliert 'Zugänglichkeit' als Grundsatz, den er anhand der drei Kategorien ausführt. Hinsichtlich des Stils fordert er 'Klarheit und Übersichtlichkeit'; zudem sollten sich die Autoren vor 'Stilisierungskunststücken' hüten. ${ }^{296}$ All dies sind rhetorische Begriffe, die für Lesbarkeit sprechen. Hinsichtlich einer thematischen Zugänglichkeit macht Becher die Erarbeitung eines Sujets geltend, das die Lebensumstände des Volkes widerspiegelt und in dessen Fokus der Protagonist steht. Der Arbeiter wird zum positiven Helden, was Becher als essentielle Voraussetzung für Zugänglichkeit wertet: 'Indem die Literatur den Zugang eröffnet für alle Gestalten des Volkslebens, wird sie auch dem Volke selbst zugänglich, das sich in ihr dargestellt [...] findet.' 297 Die Identifikation mit dem Helden liefert dem Volk den Zugang zum Text. Das Identifikationspotenzial knüpft Becher zudem an einen Erziehungsanspruch: 'Was ist ein Held: ein Vorbild, hingestellt vom Dichter, damit Tausende sich ihm nachbilden.' 298 Dies unterstreicht die Instrumentalisierung von Lesbarkeit für die politische Agenda. ${ }^{299}$

In Bechers politisch instrumentalisierten 'Sozialistischen Realismus' wird 'Zugänglichkeit' zur zentralen Metapher für Lesbarkeit. 'Zugänglichkeit' resultiert aus der Orientierung an den realen Verhältnissen des Volkes: 'Das Volk ist die beste Schule der Einfachheit', 300 Gerade von der einfachen Gestaltung verspricht sich Becher eine

\footnotetext{
293 Dass dieses Prinzip der Volksverbundenheit ein wesentliches Anliegen der DDR-Literatur war, äußert auch Wolfgang Emmerich in seiner Literaturgeschichte der DDR. Emmerich führt an, dass in diesem Kontext 'Lesbarkeit' zum zentralen Begriff der Literaturproduktion wird, vgl. Emmerich, 1989, S. 21.

294 Wie Fontane führt Becher die Unterscheidung von Wirklichkeit und Wahrheit ein. Allerdings bleiben seine Ausführungen vage, sodass nur vermutet werden kann, dass Wirklichkeit die Wiedergabe der Realität und Wahrheit die sozialistische Weltsicht meint, die es zu verbreiten gilt; vgl. Becher 1962, S. 36.

295 Vgl. ebd., S. 36f.

296 Vgl. ebd., S. 449.

297 Ebd., S. 37.

298 Ebd., S. 449.

299 Zudem sollten die Autoren vermehrt aus der Arbeiterklasse rekrutiert werden, wodurch Becher ähnlich wie Kästner Autoren und Leser als ebenbürtige und Erfahrungsschatz teilende Gruppierungen positioniert. Vgl. ebd., S. 453.

300 Ebd., S. 449.
} 
'große deutsche Kunst', die zu großen Teilen der Bevölkerung spricht. ${ }^{301}$ Durch die Größenmetapher und das Kunstattribut erhebt er Einfachheit zur Qualität. Dementsprechend wurden Werke kritisiert, die vermeintlich zu komplex konzipiert waren, um die didaktische Funktion des 'Sozialistischen Realismus' zu erfüllen. Ironischerweise war der Gegenstand dieser Debatte wie 1990 Christa Wolf. Ihr Roman Der geteilte Himmel (1963) bewegte aufgrund seiner ästhetischen Qualitäten westliche Feuilletons zu einem allmählichen Umdenken hinsichtlich der DDR-Literatur. Waren die bisherigen Werke nicht als anspruchsvolle Literatur wahrgenommen worden, so begannen die bundesdeutschen Kritiker die DDR-Literatur nun als eigenständige Literatur ernst zu nehmen. Die Literaturkritik in der DDR lobte und prämierte Wolfs Roman zwar, jedoch sah sie das Ziel der sozialistischen Umerziehung der Bevölkerung wegen einer komplexen ästhetischen Gestaltung gefährdet. ${ }^{302}$

In der westdeutschen Gruppe 47, der stilbildenden Institution der deutschen Nachkriegsliteratur, befürchtete man hingegen, dass ein zu starker Fokus auf die Didaktik zu einem ästhetischen Qualitätsverlust führen würde, worin sich gegen Ende der 1960er Jahre bereits die Diskussion von 1990 spiegelt. ${ }^{303}$ Die Gruppe formierte sich 1947 um Alfred Andersch und Hans Werner Richter, die Redakteure des politischen Journals Der Ruf, was als Indiz für die spätere Verlagerung des Kräfteverhältnisses zugunsten eines politisch-moralischen Engagements gegenüber der Ästhetik gewertet werden kann. ${ }^{304}$ Jedoch trat vor allem Andersch für eine Literatur ein, die künstlerischen Ansprüchen genügen sollte, wobei auch kommerzieller Erfolg eine Rolle spielt. Andersch urteilt in einem Brief an Richter (1954):

Es ist natürlich ein grosser Unterschied, ob ein Künstler seine Arbeit unter dem Gesichtspunkt des Erfolgs sieht, oder unter dem des Kunstwerks, d.h. ob er Erfolge haben oder seine Vision verwirklichen will. Der Anblick eines um den Erfolg ringenden Kollegen stimmt mich immer zum Hohngelächter. ${ }^{305}$

\footnotetext{
301 Ebd., S. 454; die thematische Hinwendung zum Volk und die dadurch wachsende Kunstfertigkeit thematisiert etwa Eduard Claudius in seinem Arbeiter-Roman Menschen an unserer Seite (1951). Der Maler Andrytzki gelangt erst dann zu künstlerischem Ansehen als er Aehre, den Helden des Romans, bei der Arbeit portraitiert. Vgl. Claudius 1984.

302 Für eine detaillierte Dokumentation und einen Kommentar der Debatte siehe Reso 1965.

303 Zur Gruppe 47 siehe u.a.: Arnold 2004; Arnold 1980; Kröll 1979; Neunzig 1979; Richter/Mannzen 1964.

304 Der Ruf war eine zwischen 1946 und 1949 herausgegebene, der Demokratisierung und Sozialisierung Deutschlands verschriebene kulturpolitische Zeitschrift.

${ }^{305}$ Alfred Andersch zitiert nach Arnold 2004, S. 54.
} 
Deutlich ist hier die auch bei Adorno und Horkheimer festzustellende Dichotomie zwischen künstlerischem Anspruch und finanziellem Erfolg. Wie die Frankfurter Philosophen privilegiert Andersch den künstlerischen Anspruch. Dazu nutzt er den religiösen Terminus der 'Vision', durch den er den Spott gegenüber Schriftstellerkollegen verdeutlicht, die sich intensiv um Verkaufserfolg bemühen.

Ihren Höhepunkt erreichte die Debatte um ästhetische Qualität im April 1966. Peter Handke rekurriert auf das die Nachkriegsliteratur prägende Spannungsfeld zwischen Ästhetik und Moral und zieht in seiner nachträglichen Reflexion Bilanz:

In Princeton nun mußte ich hören, wie sehr das sogenannte gesellschaftliche Engagement des Schriftstellers von den Kritikern in der Gruppe 47 an den Objekten gemessen wurde, die er beschreibt, und nicht an der Sprache, mit der er diese Objekte beschreibt. [...] Sprache ist eine Realität für sich, und ihre Realität kann nicht geprüft werden an den Dingen, die sie beschreibt, sondern an den Dingen, die sie bewirkt. Mir ist während der Tagung aufgegangen, daß formale Fragen eigentlich moralische Fragen sind. Wagt es jemand, in einer unreflektierten Form über heiße Formen zu schreiben, so erkalten diese heißen Dinge und erscheinen harmlos. Den berüchtigten Ort A in einem Nebensatz zu erwähnen, geht vielleicht an. Ihn aber bedenkenlos in jede Wald- und Wiesengeschichte einzuflechten, in einem unzureichenden Stil, mit untauglichen Mitteln, mit gedankenloser Sprache, das ist unmoralisch. ${ }^{306}$

Handkes Kritik richtet sich gleichsam gegen Rezensenten und Autoren. Zunächst attackiert Handke die in der Gruppe vertretenen Kritiker um Marcel Reich-Ranicki. Wie Böll wirft er jenen vor, beinahe ausschließlich nach inhaltlichen moralischen Kriterien und nicht nach ästhetischen Qualitäten $\mathrm{zu}$ urteilen. Sprache wird für ihn zum entscheidenden Gradmesser, wobei er für eine wirkungsästhetische sprachliche Gestaltung plädiert, mit der er ein moralisches Engagement anstrebt. Dies wird besonders im zweiten Teil des Absatzes deutlich, in dem Handke sich explizit gegen seine Kollegen wendet. Er wählt die literarische Aufarbeitung von Auschwitz als Bezugspunkt, um das Missverhältnis zwischen Moral und Ästhetik aufzuzeigen. Autoren würden das moralische Engagement - in diesem Fall die Aufarbeitung des Holocaust - in unzulängliche Handlungsverläufe und eine dilettantische stilistische Gestaltung betten und könnten dadurch der Bedeutung des Themas nicht Genüge leisten. Als Effekt stelle sich eine Verharmlosung des Gegenstands ein, was Handke als unmoralisch bezeichnet. Demnach wird für Handke eine qualitativ hochwertige Ästhetik 
zur Grundvoraussetzung für den Wirkungserfolg einer moralisch ambitionierten Literatur, was mit Manns Forderung nach dem moralisch und ästhetisch 'Guten' korrespondiert.

In der Gruppe 47 zeigt sich deutlich eine zunehmende Unzufriedenheit mit der Tendenz, dass sowohl Autoren als auch die Kritik Werke vorrangig nach moralischen Gesichtspunkten bewerten und ästhetische Kategorien hingegen außer Acht lassen. Während Mann die beiden Kategorien als Einheit etabliert, zeichnet sich in der Nachkriegsliteratur eine zunehmende Spaltung zwischen ihnen $a b$, die ein Konfliktpotenzial entwickelt, das zum zentralen Streitpunkt der Debatte um 1990 wird. 


\section{Kapitel 3: Der Literaturstreit und das literarische Programm der 'Neuen Deutschen Lesbarkeit'}

Zwischen dem Mauerfall am 9.11.1989 und der Wiedervereinigung am 3.10.1990 entbrannte in Deutschland ein Literaturstreit, der das Spannungsfeld zwischen moralisch, politischem Engagement und Ästhetik, das die Nachkriegsliteraturen beider deutscher Teilstaaten geprägt hatte, aufzulösen gedachte. Während die poetologischen Aspekte des Lesbarkeitsbegriffs in der deutschen Literaturgeschichte seit 1700 diskutiert wurden, erhielt der Terminus 'Lesbarkeit' durch das Programm der 'Neuen Deutschen Lesbarkeit' seine terminologische Fixierung und wurde in einem bis dahin nicht da gewesenen Kontext herangezogen. Die Vertreter der 'Neuen Deutschen Lesbarkeit' nutzten Lesbarkeit auf einem globalen Markt und in Konkurrenz zu den neuen Medien, um die deutsche Literatur bei Lesern im In- und Ausland erfolgreich zu machen. Dabei unternahmen sie es, den Einfluss der Frankfurter Schule auf den deutschen Literaturbetrieb zu brechen, im Anschluss an die amerikanische Postmoderne die Dichotomie zwischen E- und U-Literatur obsolet zu machen und Unterhaltung als Wirkungsabsicht zu legitimieren. Uwe Wittstock liefert mit Leselust ein grundlegendes theoretisches Werk, das bereits Martin Hielscher in seiner Kurzgeschichtenanthologie Wenn der Kater kommt - 38 deutschsprachige Autorinnen und Autoren (1996) als Standardwerk zur 'Neuen Deutschen Lesbarkeit' anführt und dadurch eine Traditionsbildung vorantreibt. Matthias Politycki, der einzige fiktionale Autor des Trios, unterstreicht mit seinen teils provokativen, plakativen und polemischen poetologischen Äußerungen, die er in seiner die Lesbarkeitsdebatte dokumentierenden Essaysammlung Die Farbe der Vokale. Von der Literatur, den 78ern und dem Gequake satter Frösche (1998) zusammenfasst, den Streitcharakter der Lesbarkeitsdebatte. Im Folgenden sollen zunächst die zeitgeschichtlichen Hintergründe des Literaturstreits umrissen werden, ehe eine fundierte Analyse des Programms der 'Neuen Deutschen Lesbarkeit' die nachhaltigen Hauptaspekte des Literaturstreits herauskristallisieren wird, um eine zeitliche und theoretische Grundlage für die in den anschließenden Kapiteln zu besprechenden Autoren und Werke zu erarbeiten.

Die Publizisten Ulrich Greiner und Frank Schirrmacher lösten den Literaturstreit mit ihrem Angriff auf Christa Wolfs autobiografisch inspirierte Erzählung Was bleibt (1990) 
aus. $^{307}$ Sie warfen Wolf vor, sich nach der Wende zum Opfer des DDR-Regimes stilisieren zu wollen. Nachdem mehrere westdeutsche Autoren wie Günter Grass oder Walter Jens Wolf vor den feuilletonistischen Angriffen in Schutz genommen hatten, wuchs sich die Debatte um deren moralisches und politisches Verantwortungsbewusstsein zu einem Streit über die Rolle der Intellektuellen in der Gesellschaft aus. ${ }^{308}$

Der Philosoph und Feuilletonist Karl-Heinz Bohrer prägte in Anspielung auf Max Weber den Terminus einer 'Gesinnungsästhetik', der die von Heinrich Böll und Peter Handke erkannte Privilegierung der politisch moralischen Aussage gegenüber der Ästhetik in der Nachkriegsliteratur suggeriert. ${ }^{309}$ Greiner und Schirrmacher nutzten dann in ihren Artikeln 'Die deutsche Gesinnungsästhetik. Noch einmal Christa Wolf und der deutsche Literaturstreit / Eine Zwischenbilanz' (1990) und 'Abschied von der Literatur der Bundesrepublik' (1990) den zeitgeschichtlichen Impuls der Wiedervereinigung und forderten eine Abkehr von der 'Gesinnungsästhetik'. Greiner argumentiert, dass sich die deutsche Nachkriegsliteratur in Auseinandersetzung mit dem Nationalsozialismus ein 'humanitäres und politisches Debet' auferlegt hätte, unter dem die ästhetische Qualität gelitten habe. ${ }^{310}$ Anders als Böll oder Handke, die jeweils im Sinne Thomas Manns eine Wiederaufwertung der Ästhetik gegenüber der politisch moralischen Aussage gefordert hatten, um ein Gleichgewicht herzustellen, dient im Literaturstreit der Verzicht auf die Gesinnungsverbreitung als Lösungsansatz.

In der Forschung variieren die Einschätzungen der Bedeutung der politischen Zäsur der Jahre 1989 und 1990 für den deutschen Literaturbetrieb. Thomas Kraft unterstreicht diese in der Einleitung $\mathrm{zu}$ seiner Essaysammlung Aufgerissen. Zur Literatur der 90er (2000) mit der Metapher eines 'drastischen Umbruch[s]'. ${ }^{311}$ Frank Finlay hingegen geht in seinem Essay 'Literary debates and the literary market since unification' (2007) von der Wiederkehr einer sich turnusmäßig wiederholenden

\footnotetext{
307 In der Erzählung behandelt Wolf einen Tag im Leben einer DDR-Autorin, die unter der Observation der Stasi zu leiden hat, vgl. Wolf 2007.

308 Zur Debatte um Christa Wolf und den deutschen Literaturstreit vgl.: Anz 1995; Deiritz/Krauss 1991; Wittek 1997; Koch 2001; kompaktere Darstellungen finden sich in Taberner 2005 und Finlay 2007.

309 Weber verwendet in seinem Essay "Der Sinn der "Wertfreiheit" der soziologischen und ökonomischen Wissenschaften' (1917) den Begriff 'Gesinnungsethik' und macht für die Sozialwissenschaften die Aufgabe geltend, Gesinnungen zwar deskriptiv darzustellen, jedoch keinen didaktischen Allgemeingültikeitsanspruch zu vertreten. Dies korrespondiert mit der Anwendung des Begriffs der 'Gesinnungsästhetik' auf die Literatur; vgl. Weber 1988. Detailliertere Ausführungen zu dieser Assoziation finden sich in Witteck 1997, S. 108f und Finlay 2007, S. 23.

310 Vgl. Schirrmacher 1990 und speziell hier Greiner 1991, S. 141.

311 Kraft 2000, S. 12.
} 
Konkurrenzsituation zwischen Politik und Ästhetik aus. ${ }^{312}$ Hier gilt es zu unterscheiden: Einerseits wurden die poetologischen Kategorien von Lesbarkeit auch hinsichtlich eines politischen Engagements in der deutschen Literaturgeschichte seit dem frühen 18. Jahrhundert immer wieder diskutiert. Andererseits ist Krafts Metapher jedoch dann valide, wenn sie sich auf die Vereinigung der beiden deutschen Literaturmärkte, den Einfluss der Globalisierung und den damit einhergehenden Entwicklungen in der deutschen Literaturlandschaft bezieht. ${ }^{313}$

Finlay hebt die Bedeutung der 'Neuen Deutschen Lesbarkeit' in diesem dynamischen Prozess hervor, indem er eine intensive Wechselwirkung geltend macht: 'The debate on the need to adopt Anglo-American models [...] was, to a greater extent than any of its precursors, both constitutive of, and constituted by, major changes in the literary market.' 314 Finlay bestätigt die für Lesbarkeit konstitutive Interdependenz von Literaturtheorie und zeitgeschichtlichem Hintergrund, wobei er hinsichtlich der Intensität eine Ausnahmestellung in den 1990ern erkennt. Die englischsprachige Literatur rückt als Vorbild in den Fokus, was er zudem an einer Dominanz von ausländischen Autoren auf dem deutschen Buchmarkt festmacht. ${ }^{315}$ Das Zusammenspiel eines Impetus von außen und eines Agens von innen initiiert im deutschen Literaturbetrieb demnach eine Abkehr von der idealistischen Tradition und insbesondere von Adornos und Horkheimers Vorbehalten gegenüber kommerziell erfolgreicher Literatur.

In dieser Konstellation avanciert der Leser zur entscheidenden Instanz im literarischen Produktionsprozess. Kraft äußert hinsichtlich der erfolgsorientierten Ausrichtung der Literatur: 'Alles hängt vom Interesse des Lesers $a b$, den es zu gewinnen gilt.'316 Dieser Impuls führte zur Suche nach ästhetischen Lösungsansätzen, um der deutschen Literatur den Erfolg bei der Leserschaft zu sichern, und bildete zwei Lager heraus. Das eine Lager repräsentierten Schirrmacher, Greiner und Bohrer, die gegen Ende des Jahres 1990 für einen Anschluss an die Tradition der europäischen Moderne und für einen auf komplexen Formen beruhenden hohen ästhetischen

\footnotetext{
312 Finlay 2007, S. 31 und 36.

${ }^{313}$ Kraft macht folgende Veränderungen geltend: 'Die rapide Globalisierung und die Vernetzung der Welt verstärkten das Primat von Wirtschaft und Medien, mit allen Vor- und Nachteilen für das literarische Leben in Deutschland' (Kraft 2000, S. 11).

314 Finlay 2007, S. 33.

315 Vgl. ebd., S. 32. Kraft attestiert der deutschen Literatur zudem wegen ihres vermeintlich ernsten und didaktisierenden Charakters wenig Chancen auf Verkaufserfolg im Ausland; Vgl. Kraft 2000, S. 15.

316 Ebd., S. 13.
} 
Anspruch plädierten. ${ }^{317}$ Um deren Literaturbegriff terminologisch greifbar zu machen, verwendet Stuart Taberner in seinem Essay "A New Modernism or "Neue Lesbarkeit"?' (2002) in Opposition zu dem im deutschen Literaturbetrieb etablierten Begriff der 'Neuen Lesbarkeit' den einer 'Neuen Moderne/New Modernism'. Er meint damit hauptsächlich ein nostalgisches Verlangen nach einer Wiederbelebung des modernen Großstadtromans, um die deutsche Literatur in eine europäische 'Hochkultur' $\mathrm{zu}$ integrieren. ${ }^{318}$ Vor allem deswegen, da sich dieser Literaturbegriff in der Praxis nicht durchsetzen konnte, ist auch der Begriff im literarischen Diskurs nicht prominent vertreten.

Auf der Gegenseite standen Wittstock, Hielscher und Politycki für das Programm einer 'Neuen Deutschen Lesbarkeit'. Wittstock liefert in Leselust (1995) sowohl eine akribische Dokumentation des '[Streits] um die Lesbarkeit in der neuen deutschen Literatur' als auch die zeitgeschichtliche und theoretische Grundlage des Programms der 'Neuen Deutschen Lesbarkeit. ${ }^{319}$ Er beginnt sein Plädoyer für die qualitative Aufwertung von Unterhaltung mit einer empirischen Beobachtung des deutschen Literaturbetriebs: viele deutsche Schriftsteller, insbesondere die jüngeren, würden zwar von der Kritik durchaus positiv aufgenommen, doch hätten die meisten von ihnen ihr Publikum verloren, das größtenteils ausländische Literatur vorziehe. ${ }^{320}$ Anders als Adorno und Horkheimer sieht Wittstock zwar keine Korrelation zwischen Verkaufszahlen und Qualität, doch schätzt er deren Sinken als bedenkliche Entwicklung ein, die auch im Ausland zu beobachten sei: 'Bedauerlicherweise gerät die deutsche Belletristik offensichtlich auch im Ausland immer mehr in den Ruf, besonders schwierig, unsinnlich und weltfern zu sein - also mehr Lesemühsal zu bereiten als Leselust zu bieten. ${ }^{321}$ Der zentrale Kritikpunkt an der deutschen Literatur ist ihre auf Komplexität beruhende Unlesbarkeit.

Mit dem Begriff der 'Sinnlichkeit' rekurriert Wittstock auf Maxim Billers Essay 'Soviel Sinnlichkeit wie der Stadtplan von Kiel. Warum die neue deutsche Literatur nichts so nötig hat wie den Realismus. Ein Grundsatzprogramm.' (1991), in dem sich

\footnotetext{
317 Genauere Analysen dieses Konzepts findet sich in Taberner 2002, S.137-139, Taberner 2005, S. 4f und Witteck 1997, S. 123-129.

318 Vgl. Taberner 2005, S. 5.

319 Wittstock 1995, S. 162.

320 Vgl. ebd., S. 7-11.

321 Ebd., S. 10.
} 
Biller gegen ein 'modernistische[s] Wirklichkeitsverbot' der Avantgarde wendet; dieses würde von Germanisten und Feuilletonisten unterstützt, die den nicht-professionellen Leser verachteten und versuchten, einer breiten Leserschaft den Zugang zum 'großen Tempel des Geistes' zu erschweren. ${ }^{322}$ Die Sakralmetapher suggeriert eine exkludierende Literaturauffassung, mit der die Autoren sowie eine elitäre Leserschaft durch eine anti-realistische Gestaltungsweise, die Wittstock durch das Adjektiv 'weltfern' ausdrückt, ihre erhabene Position zu behaupten suchen. Biller plädiert demgegenüber für einen realistischen Literaturbegriff und Verständlichkeit, um eine breite Leserschaft dadurch zu gewinnen, dass sie etwas über sich und die Welt erfahren könne. ${ }^{323}$ Die Strategie, die dargestellte Welt nahe am Erfahrungsraum des Lesers anzusiedeln, dient demnach als Mittel zur Breitenwirkung.

Breitenwirkung und Erfolg bestehen für Wittstock aus mehreren Facetten. Zum einen eröffnet er eine Dichotomie zwischen Kritik und allgemeinem Publikum, die in Deutschland seit den 1970er Jahren identifiziert werden könne: 'Zwar genießen zahlreiche Autoren nach wie vor den Respekt der Kritik, doch das Publikum haben die meisten von ihnen verloren. [...] Ihre Bücher erreichen, von einem kleinen Zirkel Eingeweihter abgesehen, niemanden mehr.' ${ }^{324}$ Wie Fiedler erkennt Wittstock eine Diskrepanz zwischen dem Geschmack der Kritik und der Leser, wobei die Weihemetaphorik auf eine exklusive Literatur verweist, die sich von der breiten Masse absetzt und vorrangig den Erfolg bei der Kritik anstrebt. Wittstock jedoch zielt ebenso auf große Leserkreise und fordert einen 'Ausbruch aus dem esoterischen Zirkel der Kenner und Connaisseurs. ${ }^{325}$ Weitere Kategorien sind dezidiert hohe Verkaufszahlen und die Nachhaltigkeit des finanziellen Erfolgs: 'Der weitaus größte Teil unserer Gegenwartsliteratur ist schon nach zwei, drei Jahren nicht mehr über den Buchhandel erhältlich, sondern nur noch in wenigen gutbestückten Bibliotheken. ${ }^{326}$ Ein letzter Aspekt ist der Erfolg im Ausland, den Wittstock an Übersetzungen aus dem Deutschen

\footnotetext{
322 Vgl. Biller 1998, S. 63 und 68.

323 Ebd., S. 69.

324 Wittstock 1995, S. 8.

325 Ebd., S. 11.

326 Ebd., S. 9. Hinsichtlich genauer Vorstellungen äußert Wittstock: 'Drei- bis viertausend Exemplare eines deutschsprachigen Romans an den Leser zu bringen, gilt heute als ein Ergebnis, das sich sehen lassen kann. Mehr wird als Erfolg gefeiert, weniger ist gerade bei jungen Autoren die Regel.' (S.8) Zum Vergleich zieht er die Verkaufszahlen der englischsprachigen Romane heran: 'Das ganze Ausmaß des Desasters wird sichtbar, sobald man sich ein paar Vergleichsgrößen vor Augen hält. Um auf einen der vorderen Plätze der Bestsellerlisten zu kommen, müssen von einem Titel einige hundert, manchmal über tausend Exemplare pro Tag verkauft werden.' (S.9).
} 
festmacht. Im Moment sei das Interesse an deutscher Literatur im Ausland so gering, dass 'immer weniger Verlage das Risiko [eingehen], sie zu übersetzen.' ${ }^{327}$

Wittstock geht im deutschen Literaturbetrieb von einem Vorurteil gegenüber kommerziell erfolgreicher Literatur aus. Dieses verdeutlicht er am Beispiel von Patrick Süskinds Weltbesteller Das Parfum (1985):

Gewöhnlich erwartet man von einem bedeutenden Autor eher, verkannt zu sein. Erfolg gilt $[\ldots]$ schnell als rechtfertigungsbedürftig. Dagegen wirkt die Behauptung, ein Schriftsteller werde von einer Minderheit hochgeschätzt, im selben sozialen Milieu gemeinhin wie ein Adelsprädikat - und zwar für diesen Schriftsteller und für jene Minderheit. Ich fürchte, hinter solchen Voreingenommenheiten steckt ein letzter Rest intellektueller Massenverachtung, schwingt etwas mit von der Sehnsucht nach kleinen, feinen Zirkeln, die über Wissen verfügen, das der bornierten Menge nicht zugänglich ist. ${ }^{328}$

Wittstock bezieht sich auf die Tradition der 'Kulturindustrie', die finanziell erfolgreichen Werken generell das Qualitätssiegel abspricht, wobei ihm zufolge ästhetische Kategorien keine Rolle spielen. Daran anschließend erkennt er eine Privilegierung von Werken mit geringen Verkaufszahlen, die im kleinen Kreis hochgelobt werden. Die zentrale Kategorie ist das soziale Prestige, das sowohl der Autor als auch die entsprechenden Leser aus der Exklusivität ziehen. Die becher'sche Zugänglichkeitssmetapher steht für eine vermeintliche soziale und intellektuelle Überlegenheit der Elite gegenüber einer breiten Leserschaft, die Wittstock in der deutschen Literaturtradition verankert sieht. ${ }^{329}$

Wittstock dekonstruiert schließlich die Tendenz des deutschen Literaturbetriebs, Misserfolg auf dem Absatzmarkt als Qualitätsbonus anzusehen. Um nach den Gründen für den mangelnden Verkaufserfolg der deutschen Literatur $\mathrm{zu}$ fragen, stellt er provokativ fünf Thesen auf, die die Schuld beim Leser suchen. ${ }^{330}$ In deren Negierung

\footnotetext{
327 Ebd., S. 10.

328 Ebd., S. 139f. Im Anschluss kritisiert er vor allem den Umgang der Germanistik mit Süskind, die den Roman kaum beachtet habe.

329 Dass dies zutrifft und der Beginn dieser Entwicklung mit dem Aufstieg eines Bildungsbürgertums im 18. Jahrhundert datiert werden kann, hat das historische Kapitel der vorliegenden Arbeit aufgezeigt.

330 Ebd., S. 12. Die Thesen lauten folgendermaßen: 1. In der heutigen vereinnahmenden Berufswelt wird der Feierabend lediglich zur Reproduktion der Arbeitskraft und nicht zum Lesen gebraucht. 2. Audiovisuelle Medien haben Bücher in die Vergessenheit verdrängt. 3. In der von Leistungsdenken beherrschten Zeit werden nur noch Sachbücher, Ratgeber oder Reiseführer, also Bücher mit einem Zweck gekauft und nicht zwecklose Literatur. 4. Die Fähigkeit der Konzentration lässt in der Leserschaft nach, sodass nur noch literarische Kurzformen rezipiert werden. 5. Bei der Rezeption von Literatur und auch Filmen wird davon ausgegangen, dass auf dem Markt nur triviale und keine anspruchsvollen Werke reüssieren können. Die Thesen und deren Widerlegungen finden sich auf den Seiten 12-16.
} 
erhellt Wittstock die Verantwortlichkeit der Autoren und nimmt sie in die Pflicht, sich einem 'Prinzip der Verführung' zu verschreiben:

An einem Winterabend lehnt sich ein einigermaßen gebildeter, gutwilliger Medienkonsument in seinem Sofa zurück, die Stehlampe brennt, die Zentralheizung rauscht leise, und vor ihm auf dem Couchtisch liegen ein sogenanntes 'gutes' Buch, ein ziemlich blutrünstiger Kriminalroman, die Tageszeitung, eine Illustrierte und der Spiegel, dazu noch die Fernbedienungen für den Videorecorder, den CD-Player und das TV-Gerät. Das ist, glaube ich, kein übertriebenes, eher ein recht alltägliches Szenario. Welche Chancen hat nun jenes 'gute' Buch, es stammt von einem deutschen Schriftsteller, die Gunst seines Besitzers auf sich zu lenken? Es hat nur eine: Es muß ihm Vergnügen machen. ${ }^{331}$

Wittstock zeichnet ein Bild der Behaglichkeit, das an die Konzeption der Gartenlaube erinnert. Es unterscheidet sich dadurch von dem der Zeitschrift, als dass es nicht dazu herangezogen wird, um die angenehme Rezeption des Textes zu spiegeln und zu unterstreichen, sondern um die gegenwärtigen ökonomischen Bedingungen darzustellen, die ein Konkurrenzverhältnis zwischen verschiedenen Genres und Medien befördern. Wie die Gartenlaube entwirft er einen Jedermann als Rezipienten, der möglichst viele Leser einschließt. Mit 'gut' verwendet Wittstock ein Attribut, das Thomas Mann in der 'Rede zum Goethejahr' als Verschmelzung von ästhetischer Qualität und moralischer Aussage etabliert. Wittstock betont in seinem Szenario die ästhetische Qualität und macht keine politisch moralische Funktion geltend. 332 Das Vergnügen etabliert er als Mittel zum Erfolg und als Bestandteil einer qualitativ ansprechenden Literatur.

Das Vergnügen ist der zentrale Aspekt von Wittstocks Einordnung der deutschen Literatur in den internationalen Kontext. Er spricht sowohl von einem 'literarische[n] Sonderweg Deutschlands' als auch von Deutschland als einer 'verspätete[n] Nation'. ${ }^{333}$ Dies macht Wittstock an zwei Punkten fest: Zum einen an einer populären Formen feindseligen Konfiguration der Autor-Leser-Beziehung. Zum anderen an einer in

\footnotetext{
331 Ebd., S. 18.

332 Wittstock wundert sich darüber, welcher Stellenwert politischen Äußerungen von Autoren in Deutschland in politischen Umbruchszeiten wie 1989/1990 zugemessen wird. Er selbst vertritt die Position, dass 'die politischen Ansichten von Schriftstellern für das Klima einer hochdifferenzierten Industriegesellschaft wahrscheinlich keine größere Bedeutung haben als, sagen wir, die entsprechenden Verlautbarungen der Faserplatten-Industrie oder der Binnenschifffahrt' (37f). Die Autoren bettet er egalitär zu anderen Berufsgruppen in die gegenwärtige Gesellschaft ein und stellt sie nicht auf ein exponiertes Podium, das sie als moralische Instanzen herausstellen würde. Später zieht er Sten Nadolnys Münchner Poetikvorlesungen (1990) heran, um sich gegen die didaktische Literatur von 'literarischen "Gurus" (als Beispiele nennt er Günter Grass und Christa Wolf) zu wenden und für eine Literatur einzutreten, die auf Unterhaltung setzt (S. 77f.). Zudem sieht er diese Idee von einer didaktischen Funktion der Literatur gegenüber der Gesellschaft als 'eine sehr deutsche [Idee]' an, was die Vorstellung eines deutschen Sonderwegs unterstreicht (S. 33).

333 Ebd., S. 24 und 25.
} 
Deutschland erst spät einsetzenden Rezeption der Postmoderne. Im Fazit fordert Wittstock eine Literatur, die sich an den 'common reader' richtet, ein Terminus aus The Life of Gray des englischen Autors Samuel Johnson (1781). ${ }^{334}$ Wittstock lobt Johnson, weil jener 'die ästhetische Urteilskraft des gewöhnlichen Lesers zu schätzen wusste [...], weil er die gewöhnlichen Leser für das Publikum hielt, an das die Literatur sich richtet. ${ }^{335}$ Wittstock definiert die 'gewöhnlichen Leser' als 'Menschen, die aus freien Stücken lesen, jenseits professioneller Notwendigkeiten oder Zwecke, die über gesunden Menschenverstand verfügen und von literaturtheoretischen Vorurteilen unverdorben sind. ${ }^{336}$ Wittstock zeichnet den Einfluss Johnsons auf Virginia Woolfs Anthologie The Common Reader (1925) und führt an, welchen Wert Woolf 'der Perspektive eines Menschen [beimaß], der [...] "mehr zum Vergnügen" liest "und kaum, um Wissen zu vermitteln oder die Ansichten anderer zu korrigieren." 337 Der Fokussierung der deutschen Literatur auf einen kleinen elitären Kreis stellt Wittstock die Privilegierung einer Leserschaft in der englischen Moderne gegenüber, deren Lektürebedürfnisse im deutschen Literaturbetrieb gering geschätzt werden. Er nutzt das Modell der anglo-amerikanischen Tradition, das einen hohen Stellenwert bezüglich der E-Literatur hat, um die E-U-Dichotomie zu unterlaufen. Der Adressat ist ein Leser, der Wittstocks 'Prinzip der Verführung' entsprechend keine professionell literarische Ausbildung durchlaufen hat, grundsätzlich dem Lesen gewogen ist und das Lesen als Teil seines Freizeitvergnügens betrachtet, ohne dabei den eigenen Nutzen oder den Nutzen anderer im Auge zu haben. Erneut stellt Wittstock damit das Vergnügen als vorrangige literarische Wirkungsabsicht ins Zentrum, die seiner Meinung nach von der deutschen Literatur kaum angestrebt wird. Dieses setzt er in Opposition zu einer didaktischen Funktion, hinsichtlich derer er zwischen einer Vermittlung von Bildungsgut und einer Umerziehung des Lesers unterscheidet. Woolf fungiert als prestigeträchtiges Vorbild, um eine didaktische Funktion zu Gunsten des Vergnügens aufzugeben und eine breite Leserschaft anzusprechen. Dadurch etabliert Wittstock ein differenziertes Lesbarkeitsprogramm, das den Kontext sowohl der deutschen als auch

\footnotetext{
334 Vgl. ebd., S. 155 und Johnson 2006, S. 184.

335 Wittstock 1995, S. 155.

${ }^{336}$ Ebd. Bei Johnson heißt es: 'for by the common sense of readers uncorrupted with literary prejudices, after all the refinements of subtilty and the dogmatism of learning, must be finally decided all claim to poetical honours' (Johnson 2006, S. 184).

${ }^{337}$ Ebd., S. 156. Im Original schreibt Woolf: 'He reads for his own pleasure rather than to impart knowledge or correct the opinions of others' (Woolf 1984, S. 1).
} 
der englischsprachigen Gegenwartsliteratur transzendiert, und eröffnet die Perspektive auf eine umfassende internationale Tradition.

Das Autor-Leser-Verhältnis der englischsprachigen Moderne dient Wittstock als Alternative zur Rezeption der deutschen Moderne in der Literatur der 1990er Jahre. Er äußert, dass der 'gewöhnliche Leser' den Versuchen der Autoren ausweiche, 'mit alten Mitteln der Moderne zu befremden, zu brüskieren oder zu belehren, also den Versuchen, ein vergangenes Verhältnis zwischen geistig vorausgeeiltem Autor und aufzuklärender Öffentlichkeit wiederherzustellen.' ${ }^{338}$ Dies kommt einer Absage an die Genieästhetik und an eine philosophisch aufklärerische Didaktik gleich. Wittstock rekonfiguriert das Verhältnis dahingehend, dass der Autor im Leser nicht eine hierarchisch unterlegene Instanz, sondern einen ebenbürtigen Partner sieht. Der Leser emanzipiert sich und macht seine eigenen Forderungen geltend, indem er eine Literatur, die seine Bedürfnisse nicht bedient, nicht liest oder kauft.

Als Hauptbedürfnis der Leserschaft benennt Wittstock ein 'Erkenntnisinteresse'; dies meint, dass die Alltagswelt des Lesers in die Romane Einzug hält, was er in der deutschen Literatur nicht gegeben sieht:

Es ist, kurz gesagt, für jene gewöhnlichen Leser nicht leicht, von deutschsprachigen Schriftstellern etwas über das eigene Leben und die eigene gesellschaftliche Rolle in Erfahrung zu bringen. Doch das Bedürfnis, das eigene [...] Leben bei der Lektüre mit entsprechenden Beschreibungen und undogmatischen Deutungsvorschlägen zeitgenössischer Autoren zu vergleichen, ist mit Sicherheit [...] berechtigt [...]. Falls aber hierzulande einen Leser das Bedürfnis [...] überkommen sollte, so ist er oft genug auf ausländische, zumal englischsprachige Erzähler angewiesen [...]. ${ }^{339}$

Wittstock formuliert ein Realismusdesiderat, mit dem er Autoren dazu auffordert, sich gezielt mit der Alltagswelt der Leser auseinanderzusetzen, wobei sie auf Didaktik verzichten und mimetisch abbilden sollen. Dem Rezipienten soll durch die Ähnlichkeit mit den Figuren ein Identifikationspotenzial gegeben werden. Der deutschen Literatur attestiert Wittstock einen Mangel an entsprechenden Werken, den er als Ursache für deren Misserfolg und die Beliebtheit der englischsprachigen Literatur bei der Leserschaft ausmacht. Insgesamt lässt sich festhalten, dass Wittstock den Verkaufserfolg der anglo-amerikanischen Literatur dazu benutzt, um die Defizite der deutschen

\footnotetext{
338 Wittstock 1995, S. 159.

339 Ebd., S. 158 und 160.
} 
Literatur zu identifizieren, während der Literaturbetrieb zur damaligen Zeit noch stark von der Theorie der Frankfurter Schule geprägt war und kommerzielle Interessen und eine leserfreundliche Ausrichtung als Missstand galten.

Neben der elitären Auffassung der Leserschaft führt Wittstock eine verzögerte Rezeption der Postmoderne als zweite Hauptursache für den Misserfolg der deutschen Literatur an. Den späten Anschluss an die Postmoderne sieht er als weiteren Grund dafür, dass die deutschen Leser ausländische Literatur bevorzugen:

Es geht $[\ldots]$ darum, $[\ldots]$ alte normative Vorstellungen beiseite zu räumen und die literarischen Spielräume zu erweitern. In anderen Weltgegenden hat diese Debatte schon vor zwei Jahrzehnten stattgefunden, und vielleicht ist ihr Ausbleiben oder ihre Verspätung hierzulande mitverantwortlich dafür, daß die Bücher ausländischer Autoren für die Leser oft attraktiver sind als die der deutschen. ${ }^{340}$

Wittstock nutzt die postmoderne Theorie, um etablierte Qualitätshierarchien zu hinterfragen und abzulösen. Dabei geht es ihm zum einen um eine Rückbesinnung auf das traditionelle Erzählen, was er in seiner Analyse von Sten Nadolnys poetologischem Werk verdeutlicht: '[Die Postmoderne] verkündet nicht neueste ästhetische Dogmen, sondern will, so Sten Nadolny, an die "vitalen Selbstverständlichkeiten des Erzählens" erinnern.' ${ }^{341}$ Zum anderen geht es Wittstock wie Fiedler darum, 'sich einiger Stoffe anzunehmen, die hierzulande gern und pauschal der Trivialliteratur zugerechnet werden.'342 Wittstock fordert eine aktive Auseinandersetzung und Aufwertung von Unterhaltungselementen, mit denen Autoren vor allem den 'gewöhnlichen Leser' ansprechen sollen. ${ }^{343}$

Zur Aufwertung von Unterhaltung rekurriert Wittstock auf prestigeträchtige Vorbilder aus der deutschen Tradition. Bertold Brechts Kleinem Organon (1948) entlehnt er die Kapitelüberschrift 'Die nobelste Funktion: Unterhaltung' und zeigt damit auf, dass Unterhaltung keineswegs exklusiv die Wirkungsabsicht der Trivialliteratur ist, sondern sich auch qualitativ ambitionierte Autoren und Werke ihrer verschreiben. Dazu eröffnet er mit Schillers Studie Über den Grund des Vergnügens an tragischen

\footnotetext{
340 Edb., S. 35.

341 Ebd., S. 59.

342 Ebd., S. 34. Als Beispiele nennt er Seeräuber und Abenteuergeschichten in Nadolnys Entdeckung der Langsamkeit (1983), den historischen Roman in Süskinds Parfum, die Detektivgeschichte in Christoph Ransmayrs Die letzte Welt (1988) sowie die Liebesgeschichte in Bodo Kirchhoffs Infanta (1990), vgl. ebd., S. 34.

${ }^{343}$ Die Affinität der Postmoderne zu Unterhaltung benennt Wittstock als eine Ursache dafür, dass Deutschland sich den postmodernen Entwicklungen lange verschlossen hat. Vgl. ebd., S. 53.
} 
Umständen (1792) und Kants Kritik der Urteilskraft (1790) eine Dichotomie zwischen einer 'Poetik der puren Vergnüglichkeit' und einer Literatur, 'die Vernunft und Sinnlichkeit der Leser auf gleiche Weise anspricht.' 344 Wittstock spielt keinen qualitativ hochwertigeren, mit einer prestigeträchtigeren Funktion kombinierten, gegen einen qualitativ minderwertigeren Unterhaltungsbegriff aus, sondern versucht ein Umdenken hinsichtlich der qualitativen Einordnung von Unterhaltung einzuleiten. Das Publikum zu unterhalten, zählt für ihn zu den 'Wesensmerkmalen der Kunst' und daher müsse in Deutschland mit dem Missverständnis aufgeräumt werden, dass große Werke ausschließlich an den Intellekt appellierten und nur ausgebildeten Kennern begreiflich seien. $^{345}$ Unterhaltung avanciert $\mathrm{zu}$ einer Grundfunktion, die ungeachtet des Qualitätsanspruchs eines Werkes gewahrt werden soll.

Dass Wittstock durchaus hohe ästhetische Ambitionen verfolgt, zeigt die Etablierung der Schwierigkeit, eine Kombination von qualitativem Anspruch und Unterhaltung in der Praxis umzusetzen:

Die Forderung, Literatur müsse [...] Vergnügen bereiten, legt es [...] nicht darauf an, das Niveau der Literatur zu senken. Es geht vielmehr darum, daß ein literarisch ernst $\mathrm{zu}$ nehmendes Buch neben großen ästhetischen Qualitäten auch Unterhaltungsqualitäten haben sollte. Es geht darum, daß die künstlerischen Ansprüche verschmelzen mit dem Anspruch, dem Leser Vergnügen zu bereiten. Folglich wird nicht weniger von den Autoren verlangt, sondern mehr [...]. ${ }^{346}$

Wittstock bietet den Autoren den Anreiz einer literarischen Herausforderung. Dadurch, dass er den diffizilen Charakter eines solchen Literaturprogramms unterstreicht und eine schriftstellerische Meisterschaft voraussetzt, kann sein Unterhaltungsbegriff als qualitativ hochwertig gelten. ${ }^{347}$ Die Verschmelzungsmetapher deutet auf einen Literaturbegriff, in dem Unterhaltung und Ästhetik einander in einer hochwertigen Mischform bedingen, die einen dynamischen Prozess vermittelt, welcher am Ende zu einer Einheit führen soll.

\footnotetext{
344 Ebd., S. 19.

345 Vgl. ebd., S. 20. An selber Stelle äußert er zudem, dass seiner Auffassung nach Texte auch durch ernste Themen unterhalten können und auch Leser, die schwierige und für Nicht-Kenner unzugängliche Texte bevorzugen, befriedigt werden sollen.

346 Ebd., S. 22.

${ }^{347}$ Er attestiert einem Autor, der es schafft beiden Anforderungen gerecht zu werden: 'Er is anderen, denen das nicht gelingt, überlegen, denn er fügt seiner Arbeit eine wesentliche Dimension hinzu.' (Ebd., S. 20).
} 
Nach der Etablierung von Unterhaltung als Qualität widmet sich Wittstock der Definition. Laut ihm heißt unterhaltsam zu sein, 'mit literarischen Mitteln beim Publikum Interesse für ein Thema, Anteilnahme an einer Figur, Neugier auf ein Geschehen oder auch Lust an einem ungewöhnlichen Sprachspiel zu wecken und wachzuhalten.' ${ }^{348}$ Wittstock evoziert sein 'Prinzip der Verführung', in dessen Kontext Unterhaltung als zugrundeliegende Wirkungsabsicht fungiert, verweist aber zudem explizit auf literarische Mittel. Aus dem Zitat lassen sich zwei Hauptbereiche ableiten, auf die diese Mittel abzielen: Handlung und Sprache. Dabei wird deutlich, dass durch die unterhaltsame Gestaltung sowohl die Sinne/Emotionen als auch der Intellekt angeregt werden sollen, um den Leser so nachhaltig an die Lektüre eines Werkes zu binden.

Um 'Intellekt' und 'Sinnlichkeit'/'emotionale Aspekte' zugleich zu befriedigen, greift Wittstock auf Fiedlers theoretische Werke zurück. Er zieht erneut eine Dichotomie zwischen 'literarischen Qualitäten' und 'Unterhaltungsqualitäten' und die Strategie der postmodernen Doppelcodierung heran:

[Fiedler erwartet] von zeitgenössischen Schriftstellern, daß sie - wie die zeitgenössischen Leser - mehrere Sprachebenen zugleich beherrschen, daß sie Anspruchsvolles wie auch Triviales parallel zu formulieren verstehen (wobei trivial genaugenommen 'für jedermann zugänglich' meint und erst im übertragenen Sinn 'alltäglich, gewöhnlich, platt'). Charles Jencks nannte das 'Doppelcodierung': Im Zeitalter der Postmoderne dürfe sich ein Kunstwerk nicht auf eine Sprachebene [...] beschränken. Es müsse vielmehr die Kenner wie die große Öffentlichkeit ansprechen, müsse elitäre Bedürfnisse genauso befriedigen wie populäre und moderne Elemente genauso aufnehmen wie traditionelle. ${ }^{349}$

Wittstock bezieht die Doppelcodierung einerseits auf intertextuelle Bezüge, die er als Referenzen auf moderne und traditionelle Elemente anführt. Dies zeigt, dass die postmoderne Abkehr von der Avantgarde auch in Wittstocks poetologischem Instrumentarium verankert ist. Andererseits bezieht Wittstock die Doppelkodierung auf die Stilistik. Er meint eine vom Realismus inspirierte Stilvielfalt, die unter der Prämisse des rhetorischen Prinzips der Angemessenheit adäquat eine literarische Kunstsprache und Alltagssprache einsetzt. ${ }^{350}$ Während die Kunstsprache dem Einfluss der komplexen Moderne zuzuordnen ist und auf den elitären Leser zielt, steht Alltagssprache für

\footnotetext{
348 Ebd., S. 22.

349 Ebd., S. 48.

350 Wittstock führt die Moderne wie auch Biller als hauptverantwortlich für die Abkehr von realistischen Erzählformen und einer daraus resultierenden 'Kluft' zwischen Autoren und Lesern im deutschsprachigen Raum an; vgl. ebd., S. 50.
} 
populäre Elemente, mit denen eine breite Masse angesprochen werden soll. In dieser Einteilung spiegelt sich eine Stigmatisierung von vermeintlich trivialen Sprachformen. Durch die Definition des Terminus' 'trivial' etabliert Wittstock den aus Bechers Literaturtheorie bekannten Begriff der 'Zugänglichkeit' als prestigeträchtigeres Synonym, wodurch er sein Lesbarkeitsdesiderat bekräftigt. Die Nähe zur Sprache der Leser bezeichnet Wittstock als 'unausgesprochenes Einverständnis', was an Jonathan Franzens Vertrags-Metapher erinnert und ein egalitäres Verhältnis vermittelt: '[Der Autor] will seine Leser weder belehren noch sie durch besonders kostbare Ausdrucksmittel einschüchtern. ${ }^{351}$ Durch die Ablehnung einer didaktischen Funktion geben Autoren ihre überlegene Position gegenüber den Lesern auf. ${ }^{352}$ Eine zu anspruchsvolle Sprache, die er in der Form des ornatus vorbringt, verwirft Wittstock im Sinne von Aristoteles' Rätsel-Metapher, um die Balance zwischen leichter Lesbarkeit und ästhetischer Ambition zu wahren. Dabei setzt er zudem auf 'sprachliche Präzision', was im rhetorischen Stilprinzip der brevitas verankert ist.

Der Einfluss der klassischen Rhetorik sowie der aristotelischen Mimesis-Tradition wird zudem in Wittstocks Romananalysen deutlich sowie in seiner Ablehnung der Werke der älteren Generation, die durch Christa Wolf und Peter Handke vertreten wird. Für Nadolnys Roman Selim oder Die Gabe der Rede (1990) macht Wittstock geltend, dass der Roman auf einer Metaebene anhand der Hauptfigur des Redners einen Übergang von der Rhetorik zum Romanschreiben darstellt: 'an die Stelle des Redens und der Rhetorik treten für ihn das Erzählen und die Poetik.' ${ }^{353}$ Wittstocks Begriff des Erzählens speist sich wiederum aus den poetologischen und rhetorischen Werken von Aristoteles:

Die Inspiration verlangt [...] nach Organisation: Der Romancier muß sie nach rationalen Kriterien durchformen, um eine angemessene und in sich konzise Architektur der zu entwickelnden Geschichte zu entwerfen. Für einen Schriftsteller wie Nadolny, der nicht zufällig einen Rhetor zur Hauptfigur eines Romans gemacht hat, spielt hierbei die Absicht, seine Geschichte einem größeren Publikum zu vermitteln, sicher eine beträchtliche Rolle. [...] Hat der Autor seine Geschichte bedachtsam konzipiert, entwickelt sie eine innere Logik und Zwangsläufigkeit. ${ }^{354}$

\footnotetext{
351 Ebd.

352 Dies untermauert Wittstock, indem er den Leser als 'Mitspieler' und 'Koproduzenten' bezeichnet, was an Umberto Ecos Bild des 'Komplizen' erinnert, das Wittstock später selbst verwendet und von einer Begegnung 'auf Augenhöhe' spricht; vgl. ebd., S. 83 und 123 f sowie Eco 1986, S. 59f.

353 Wittstock 1995, S. 86

354 Ebd., S. 72.
} 
Wittstock konstatiert zwei für die Lesbarkeitsdebatte wichtige Kriterien. Zum einen präferiert er eine Handlungsführung, die im Sinne der aristotelischen Theorie eine akribisch erzählte Geschichte logisch aufbaut und konsequent ausführt. Dies verweist auf das traditionelle Erzählen. Zum anderen sieht er eine solche Handlungskonzeption als Erfolgsgarantin dafür an, eine möglichst große Breitenwirkung zu erzielen, was hier meint, für eine möglichst große Leserschaft verständlich zu sein.

Bei seinen Ausführungen zum Werk des Autors Ulrich Woelk kombiniert Wittstock die Einheit der Handlung mit einer Einheit der Figuren. Er lobt dessen stringente Handlungsführung und außerdem, dass es Woelk gelingt, 'prägnante und klar umrissene Figuren zu präsentieren, die den Leser miterleben lassen.' ${ }^{355}$ Die möglichst kurze und klare Darstellung spiegeln auf der Figurenebene die rhetorischen Stilprinzipien der brevitas und der claritas, die als Mittel zur Anschaulichkeit fungieren. Das Miterleben teilt dem Leser jedoch eine weitaus aktivere Rolle zu. Es impliziert, dass der Autor bei der Abfassung des Textes auf eine Partizipation des Lesers setzt und seine Figuren dementsprechend gestaltet.

In Wittstocks Lesbarkeitsprogramm stellt die Rekonfiguration des Autor-LeserVerhältnisses die zentrale Kategorie dar, aus der sich alle weiteren ästhetischen Überlegungen ableiten. Er proklamiert in der deutschen Nachkriegsliteratur einen Übergang von einer 'radikale[n] Produktionsästhetik', in der die 'Frage nach der Rezeption [...], wie eindringlich etwas an die Leser weitergegeben wird, [...] nur eine sehr untergeordnete Rolle' spielt, zur 'Kommunikation'. ${ }^{356}$ Der Autor beansprucht für sich zunächst die zentrale Rolle in der Literaturproduktion, missachtet die Bedürfnisse seines Publikums und bemüht sich nicht, seine Texte leserfreundlich zu gestalten. Durch den Einfluss der Postmoderne avanciert der Leser zum emanzipierten Partner, auf den die ästhetischen Überlegungen während der Textgenese ausgerichtet werden. Wittstock wählt zwei etablierte und fundierte Referenzsysteme, die für die angestrebten Ziele stehen: die anglo-amerikanische und die aristotelische Tradition. Erstere dient der Legitimierung von Breitenwirkung als Desiderat und von Unterhaltung als Qualität, im Zuge derer dem Leser eine tragende Rolle im Produktionsprozess zugeteilt wird. Die aristotelische Tradition fungiert als Gegenpol zu der von Wittstock kritisierten 'Produktionsästhetik' und liefert ein System dafür, möglichst viele Rezipienten für den 
Text zu gewinnen, indem es eine Balance aus Verständlichkeit und hohen Ambitionen herstellt. Wittstock kreiert in Leselust eine umfassende theoretische Grundlage für den Lesbarkeitsbegriff zum Ende des 20. Jahrhunderts. Er erschließt zeitgeschichtliche Impulse, verweist auf die stabile Basis der Rhetorik und die Postmoderne als unmittelbaren zeitgenössischen Einfluss und eröffnet zudem eine Perspektive auf eine große internationale Tradition der Lesbarkeit.

Während die theoretischen Ansätze von Hielschers Wenn der Kater kommt im Wesentlichen mit denen von Wittstocks Leselust kongruieren, kann Hielschers Kurzgeschichtenanthologie als früher Indikator für den Erfolg von Wittstocks poetologischen Forderungen gelesen werden. Er argumentiert auf derselben Basis wie Wittstock: die deutsche Literatur habe im In- und Ausland ihre Leser verloren, was auf eine Stigmatisierung von Breitenwirkung und eine damit einhergehende Missachtung der Bedürfnisse der Leser zurückzuführen sei; die Debatte in den 1990er Jahren habe jedoch eine Reevaluierung der Einstellung gegenüber Lesbarkeit angestoßen:

Wir scheinen nun in eine Phase gekommen zu sein, da die deutschsprachige Gegenwartsliteratur, was die jüngere Generation anbelangt, [...] wieder offener geworden ist und auch ein größeres Publikum zu interessieren vermag. [...] Die in jüngster Zeit erschienenen Bücher von [u.a. Helmut Krausser] erweckten großes Interesse und erreichten oft beachtliche Verkaufszahlen, ohne daß auch nur eines dieser Bücher dafür Zugeständnisse an die Trivialität hätte machen müssen. ${ }^{357}$

Wie Wittstock und Politycki versteht Hielscher die Generationenfrage zentral. Die Jungen erscheinen als literarische Bewegung, die erfolgreich eine Akzeptanz von lesbarer Literatur eingeleitet und damit Breitenwirkung erreicht hat, während der Umschwung bei den älteren Vertretern des Literaturbetriebs noch keine Resonanz zeigt. Breitenwirkung schließt für Hielscher dezidiert kommerziellen Erfolg ein. Diesen setzt er zur Qualität in Bezug, macht einen künstlerischen Anspruch geltend und entkräftet somit Adornos und Horkheimers Vorurteil, dass sich nur triviale Werke gut verkaufen und von der breiten Masse positiv aufgenommen würden.

Während Wittstock vor allem die theoretische Seite des Lesbarkeitsbegriffs abarbeitet, stellt Hielscher dem Charakter einer Kurzgeschichtenanthologie 
entsprechend den Aktivismus von Autoren als notwendigen Impuls aus der Praxis heraus, um eine publikumsaffine Literatur durchzusetzen. Vor den Entwicklungen der 1990er hätten sich viele Autoren davon abschrecken lassen, ein solches Literaturprogramm praktisch umzusetzen: 'Eine lustvolle, neugierige, kluge und an dem, was die tatsächlichen Menschen eigentlich bewegt, interessierte literarische Erkundung findet unter den Vorzeichen von Angst und intellektuellem Hochmut zu selten statt. Auch dies ändert sich gerade. ${ }^{358}$ Ein zu starkes Eingehen auf die Interessen des Publikums ist bei vielen Autoren demnach verpönt. Hielscher definiert 'Angst' nicht genauer, aus dem Kontext kann jedoch davon ausgegangen werden, dass er darunter die Furcht vor einer Stigmatisierung durch den Literaturbetrieb versteht. Die Nähe zum Publikum mindert demnach den künstlerischen Wert, worin sich Adornos und Horkheimers theoretische Ausführungen zur 'Kulturindustrie' niederschlagen. Hielscher koppelt dies an die Furcht vor dem Verlust des intellektuellen Status, die ambitionierte Autoren davor zurückschrecken lässt, publikumswirksam zu schreiben, wobei der Intellekt als Distinktionsmerkmal fungiert, das den Status des Autors und seines Werkes aufwertet. Der von Hielscher diagnostizierte Umschwung besteht darin, dass Autoren diese Vorurteile überwinden und vermehrt nach einer Literatur suchen, die ein intellektuelles Sujet für breite Massen lesbar macht und die Interessen des Publikums auf ansprechende Weise verarbeitet.

Wirkte Wittstock vornehmlich als Theoretiker und Hielscher als kommentierender Chronist, so bemühte sich Politycki, der einzige Romanautor des Trios, um die Definition konkreter ästhetischer Parameter zur praktischen Umsetzung von Lesbarkeit. Aufgrund des oft simplifizierend provokativen Charakters seiner poetologischen Äußerungen können jene allerdings eher als literaturpolitische Strategie denn als fundierte poetologische Auseinandersetzung mit Lesbarkeit gelten, die den Konfrontationscharakter der Lesbarkeitsdebatte unterstreichen. ${ }^{359}$

Den Grundkonflikt der deutschen Debatte in den 1990er Jahren benennt er in Die Farbe der Vokale an Wittstock anknüpfend als Übergang von der Moderne zur

\footnotetext{
358 Ebd., S. 321.

359 Dies spiegelt sich in der teils martialischen Terminologie, die er zur Beschreibung der Debatte heranzieht. Er spricht von einem 'selektiven Wettstreit', 'Schlachten', 'Frontlinien' und 'Stellungskrieg'; vgl. Politycki 1998 f, S. 6 und Politycki 1998 g, S. 52f.
} 
Postmoderne. ${ }^{360}$ Anders als Wittstock, der den Wandel hauptsächlich an die Unterhaltung knüpft, assoziiert Polyticki diesen mit einem Umdenken hinsichtlich der Textkomplexität. ${ }^{361}$ Er macht geltend, dass in der Moderne 'die literarische Leistung eines Textes vornehmlich aus dessen Unverständlichkeitsquotienten errechnet' wurde; in der Postmoderne hingegen liege der Fokus darauf, 'gute, lesbare Texte' zu produzieren. ${ }^{362}$ Politycki bringt künstlerische Qualität wie Mann oder Wittstock mit dem Adjektiv 'gut' zum Ausdruck, wobei die Interpunktion zu beachten ist. Während ihn Wittstock zur qualitativen Legitimierung von Unterhaltung anführt, verweist Polityckis Kommasetzung auf einen Literaturbegriff, der Lesbarkeit nicht als qualitätsexkludierend, sondern als immanente Qualität auffasst. Lesbarkeit wird so zum poetologischen Bestandteil ästhetisch ambitionierter Literatur. Dass dies durchaus auch für gewisse Strömungen und Vertreter der Moderne gilt, hat das historische Kapitel der vorliegenden Arbeit aufgezeigt, sodass Polityckis Gegenüberstellung wenig differenziert erscheint.

Politycki kritisiert drei Institutionen des deutschen Literaturbetriebs dafür, dass sie die in der Praxis vorangetriebenen Entwicklungen nicht fördern, wobei die breite Ausrichtung den umfassenden Charakter der angestrebten Wende in der deutschen Literatur hervorhebt: die Verlage, die Germanistik und das Feuilleton. Im Hinblick auf die Verlage lanciert er exemplarisch eine Kritik am Suhrkamp Verlag. Dessen Verlagsprogramm, dem er die zentrale Rolle bei einem vermeintlichen Beharren der Nachkriegsliteratur auf dem 'Hochliterarischen' zuschreibt, stellt er Wittstocks Plädoyer für Unterhaltung und Lesbarkeit gegenüber. ${ }^{363}$ Politycki fokussiert insbesondere das Vergnügen. Während die Suhrkamp Autoren überwiegend die Didaktik anstrebten und die deutsche Literatur deshalb ihre Leser verloren habe, lobt er Wittstocks 'Vorstoß gegen die Langeweile'. ${ }^{364}$ Durch die Schlachtenmetaphorik betont Politycki den Kampfcharakter der Debatte und macht ebenfalls in militärischer Metaphorik eine von

\footnotetext{
360 Vgl. Politycki 1998 f, S. 8.

361 Politycki macht den Umschwung ebenfalls daran fest, dass eine junge Autorengeneration 'dem Leser Vergnügen bereiten' möchte, was er in direkten Gegensatz zu einer engagierten Nachkriegsliteratur setzt und dadurch das delectare dem docere vorzieht. Politycki 1998 c, S. 75. Auch das movere spielt für Politycki eine Rolle. Er macht geltend, dass die Nachkriegsliteratur wegen ihres Fokus auf den Intellekt ihre Leser verloren habe und fordert: 'Auch E-Literatur muß sein wie Rockmusik', die 'unter die Haut (und nicht etwa nur unter die Hirnhaut) und von dort "ins Blut"” geht. Das Bild der 'Hirnhaut' attackiert das ausschließliche Bedienen von intellektuellen Bedürfnissen. Wie Biller und Wittstock betont Politycki den sinnlichen Einfluss von Literatur und etabliert neben der Unterhaltungsfunktion auch das movere als Wirkungsabsicht. Politycki 1998 e, S. 69.

362 Politycki 1998 b, S. 49.

363 Politycki 1998 g, S. 51.

364 Vgl. ebd., S. 55 und 60.
} 
Wittstock initiierte 'kulturelle Wachablösung' geltend, die die Autoren durch den Anschluss an die Postmoderne im Verlagswesen umsetzen sollten. ${ }^{365}$

Die Germanistik attackiert Politycki wegen eines vermeintlichen Stigmas gegenüber einer Literatur, die sich der Lesbarkeit verschreibt. Er geht von einer Präferenz für komplexe Werke aus, die er folgendermaßen zu widerlegen sucht: 'Auch in der Literatur ist das Einfache oft viel schwerer zu gestalten [...] als das Schwere, das Komplizierte'. ${ }^{366}$ Politycki wertet die Qualität des einfachen Stils dadurch auf, dass er ihn wie Wittstock als anspruchsvolle schriftstellerische Aufgabe etabliert. ${ }^{367}$ Mit dieser Strategie will er die Germanistik dazu bewegen, sich fundiert mit der von Lesbarkeit inspirierten deutschen Gegenwartsliteratur auseinanderzusetzen und $\mathrm{zu}$ deren theoretischer Grundierung beizutragen. ${ }^{368}$ Die Germanistik fungiert als prestigeträchtige Institution, die durch eine Theoriebildung zur Legitimierung und Etablierung von Lesbarkeit beitragen soll.

Wie der Germanistik attestiert Politycki der Kritik einen Mangel an Parametern, um auf Lesbarkeit gründenden Werken angemessen zu begegnen: 'Die angebliche Krise der deutschen Literatur ist in Wirklichkeit eine Krise der deutschen Literaturkritik; eine kohärente literarische Ästhetik [...] unsrer neuesten Literatur liegt noch nicht mal in Ansätzen vor [...]. ${ }^{369}$ Politycki artikuliert das Verlangen, ein literarisches Programm durch eine umfassende theoretische Fundierung $\mathrm{zu}$ legitimieren, durch die das Programm der 'Neuen Deutschen Lesbarkeit' als die dominierende literarische Ästhetik der Gegenwart etabliert werden soll. Das Fehlen von adäquaten Analysekategorien habe bewirkt, dass die Kritik die entsprechenden Werke nicht an ein großes Publikum weiterempfehle, worin sich deutlich Polityckis Verlangen nach Publikumserfolg spiegelt.

Dem Anspruch auf eine umfassende Ästhetik der Lesbarkeit kann Politycki jedoch selbst nicht gerecht werden. In seiner 1997 gehaltenen Münchner Poetikvorlesung, seiner zentralen poetologischen Arbeit, formuliert er zwar das Ziel, diese mit einer theoretischen Basis versehen $\mathrm{zu}$ wollen, doch erkennt er, dass er lediglich

\footnotetext{
365 Vgl., ebd., S. 55.

366 Politycki 1998 d, S. 36.

367 In diesem Kontext führt er den Einfluss der Frankfurter Schule an. Die Betonung von intellektuellen Qualitäten der Literatur habe zu der Annahme geführt, dass die deutsche Nachkriegsliteratur klüger als ihre Leser sei und sie daher nicht am Geschriebenen teilhaben lasse. Autoren hätten 'Verkomplizierungstechniken' angewandt, die nur dafür bezahlten Kritikern 'Entschlüsselungsglück' bereitet hätten. Vgl. Politycki 1998 e, S. 68f.

${ }^{368} \mathrm{Vgl}$ Politycki 1998 d, S. 35f.

369 Ebd., S. 26.
} 
'Vorüberlegungen' liefern kann. ${ }^{370}$ Diese basieren auf der von Politycki in der Einleitung angeführten Prämisse, als Autor Vergnügen daran zu haben, dem Leser nicht 'zur Last' fallen zu wollen und Lesbarkeit als eine 'Wirkungsästhetik' zu sehen, welche er mit Roland Barthes folgendermaßen definiert: 'Sich eine Ästhetik ausdenken [...], die restlos [...] auf der Lust des Konsumenten beruhte [...]: die Folgen wären enorm, vielleicht sogar umwerfend [...]. ${ }^{371}$ In den Begriffen des Vergnügens und der Lust findet sich Wittstocks Terminologie wieder, die Barthes ebenso wie Wittstock dazu heranzieht, Autoren darauf $\mathrm{zu}$ verpflichten, sich des Unterhaltungsbedürfnisses des Publikums anzunehmen, wodurch beide den Leser emanzipieren. Polityckis Rekurrieren auf Barthes dient der Legitimation durch ein prestigeträchtiges Beispiel aus der Tradition und dem Anschluss an internationale Strömungen. Es wird deutlich, dass die Hinwendung zum Leser nicht als Qualitätsschmälerung angesehen wird, sondern als Chance, neue Wege für die Literatur zu erkunden.

Zur konkreten literarischen Umsetzung schlägt Politycki in seiner Vorlesung zunächst das bereits zu Beginn der vorliegenden Arbeit analysierte Prinzip einer 'Simplifizierung bei gleichzeitiger Verkomplizierung' vor. Diese mittlere Position benennt er durch die Metapher 'Kalbfleisch mit Reis', die er zwischen Hummern und Burgern als kulinarische Metaphern für E- und U-Literatur anführt. ${ }^{372}$ Von beiden Kategorien sollen jeweils wesentliche Aspekte beibehalten werden:

Von [der E-Position], daß die höchste Form des Vergnügens niemals auf direktem, schnellstmöglichem Weg erschrieben bzw. erlesen werden kann, sondern gegebenenfalls auch eines phasenweisen, eines erheblichen Unvergnügens bedarf, um - im Rückblick auf den gesamten Text - eine viel höhere Befriedigung zu erzielen als diejenige, die man beim Aufklappen einer BigMäc-Schachtel sofort erhält: "Sieh an, ein BigMäc, wer hätte das gedacht". Von der andern, der UPosition, ist zu bewahren, daß sich ein Genie nicht länger durch schiefe Metaphern, konfuse Gedanken und verschrobnen Satzbau definiert, sondern durch Kürzen, Feilen, Wegwerfen, mithin: durch eine professionelle Einstellung gegenüber seinen Produkten, durch ein Selbstverständnis, das sich eher am Dienstleistungsgewerbe orientiert als an dem des Weltweisen, der jeden Bücherherbst mit ein paar frischen Abstrusitäten aufwartet. ${ }^{373}$

\footnotetext{
370 Vgl. ebd., S. 34.

${ }^{371}$ Die Stelle stammt aus Barthes 1973, S. 94. Bei Politycki 1998 f, S. 9.

372 Vgl. Politycki 1998 d, S. 28f.

373 Ebd., S. 38.
} 
Die vom E-Pol beizubehaltenden Elemente lassen sich dem Oberbegriff der Verkomplizierung zurechnen, die eine nachhaltige, durch die vertikale Metaphorik exponierte, Form des Vergnügens fördern soll, die sowohl dem Autor als auch dem Leser intensive Arbeit abverlangt. In diesem Sinne bleibt das von Politycki eigentlich abgelehnte Vorurteil, dass nur Komplexes qualitativ hochwertig sei, zu einem gewissen Grad bestehen, was seine sonstige aggressive Ablehnungshaltung unterläuft und auf eine vorsichtige, konservative Anknüpfung an prestigeträchtige Parameter hindeutet. Diese werden durch das Prinzip des aptum gefiltert, sodass sie das Vergnügen des Lesers nicht schmälern, sondern intensivieren. Im Vergleich zum unmittelbaren Vergnügen des U-Pols soll eine Spannung aufgebaut werden, die den Leser solange an den Text bindet, bis er mit dem endgültigen Vergnügen belohnt wird. Die Elemente des U-Pols fungieren bis dahin als simplifizierende Aspekte. Politycki strebt eine Neuwertung des Geniebegriffs an, in der Autoren sich nicht als vermeintliche intellektuelle Elite durch Komplexität gegenüber der Leserschaft zu exponieren suchen, sondern durch ihre Fähigkeit, Texte auf anspruchsvolle Weise für Leser zugänglich zu gestalten. Der Dienstleistungsaspekt verdeutlicht den sozioökonomischen Hintergrund, welcher den Leser als Endpunkt der literarischen Produktion aufwertet und die Produktherstellung an seinen Bedürfnissen ausrichtet. Als konkrete Mittel führt Politycki Methoden an, die um die rhetorischen Kategorien der brevitas, des aptum, der Sprachrichtigkeit und des stringent logischen Aufbaus kreisen. Wenn er auch im Kontext des virulenten Literaturstreits eine polemisch vereinfachte Charakterisierung des 'Genies' vornimmt, so ist doch sein Verweis auf eine Fehlverwendung von Metaphern insofern bedeutsam, als diese bereits seit Aristoteles als Ursache dafür gilt, dass Texte zu Rätseln werden. Durch die Betonung der vom Autor zur praktischen Umsetzung benötigten Fähigkeiten etabliert Politycki das Lesbarkeitsprogramm als qualitative Herausforderung.

Im Vergleich zu Wittstock und Hielscher erscheint Politycki sowohl gegenüber der Gegenseite als auch gegenüber der eigenen Seite als aggressiver. So teilt er zwar Wittstocks theoretische Forderungen, doch könne er dessen praktische Beispiele nicht nachvollziehen, da er sie der 'Trivialliteratur' zurechne. ${ }^{374}$ Dies unterstreicht ebenso die Dynamik der in der Praxis vorangetriebenen Entwicklungen des Lesbarkeitsbegriffs wie 
den Mangel an soliden Parametern, um der Lesbarkeit eine theoretische Grundlage zu schaffen und eine fundierte Auseinandersetzung mit ihr $\mathrm{zu}$ ermöglichen. Zudem kritisiert Politycki Wittstock dafür, lediglich die E-Schriftsteller auf Unterhaltung und nicht auch umgekehrt die Unterhaltungsschriftsteller auf Techniken der E-Literatur verpflichten zu wollen. ${ }^{375}$ Dies erscheint in Bezug auf die generelle Tendenz zur Mitte als konsequente Forderung. Für das Programm der 'Neuen Deutschen Lesbarkeit' lässt sich festhalten, dass deren poetologische Ansätze sowohl in ihrer Terminologie als auch in ihren Inhalten Aspekte aus der Tradition des Lesbarkeitsbegriffs aufgreifen. Sie sticht allerdings insofern heraus, als sie in einem zeitgeschichtlichen Kontext operiert, der sich aufgrund seiner globalen ökonomischen sowie künstlerischen Ausrichtung radikal von der Einbettung vergangener Lesbarkeitsdiskurse unterscheidet. Der Einfluss der Postmoderne ermöglichte es Wittstock, Hielscher und Politycki, Lesbarkeit und Unterhaltung zum ersten Mal um ihrer selbst willen zum poetologischen Desiderat und zur primären Wirkungsabsicht in der deutschen Literatur zu deklarieren.

Gegen Ende der 1990er und in den frühen 2000er Jahren tauschten sich die Vertreter der 'Neuen Deutschen Lesbarkeit' weiterhin hinsichtlich der Implementierung von Lesbarkeit aus, wobei unter ihnen Uneinigkeit herrschte, inwiefern die Werke ihrem poetologischen Programm entsprachen. Besonders entbrannte diese Debatte an den vielfach verkauften Werken der Strömungen des 'Fräuleinwunders'376 und der 'Popliteratur' 377.

Hielscher resümiert in seinem Essay 'Geschichte und Kritik. Die neue Lesbarkeit und ihre Notwendigkeit' (2001), dass im Literaturstreit der Aktivismus für Lesbarkeit eine 'ins Reaktionäre gewendete Interpretation der Ästhetik Adornos' aufgebrochen habe. ${ }^{378}$ Adornos und Horkheimers Abneigung gegen Breitenwirkung, die Hielscher dadurch definiert, dass Bücher 'verkauft und gelesen' werden, sei durch das Zelebrieren der Popularität der Literatur in den Medien abgelöst worden. ${ }^{379}$ Dies signalisiert, dass

\footnotetext{
375 Vgl. Politycki 1998 c, S. 77.

376 Den Begriff prägte Volker Hage im Spiegel vom 22. März 1999. Er bezieht sich auf eine Gruppe von jungen und erfolgreichen deutschen Autorinnen. Als Vertreterinnen nennt Hage u.a. Karen Duve, Judith Hermann und Zoë Jenny. Vgl. Hage 1999.

377 Gemeint ist explizit eine literarische Strömung im Deutschland der 1990er. Als Ausgangspunkt kann Christian Krachts Roman Faserland (1995) gelten. Weitere Vertreter: Benjamin von Stuckrad-Barre und Benjamin Lebert. Eine umfassende Auseinandersetzung mit der deutschen Popliteratur der 1990er liefert Ernst 2001.

378 Vgl. Hielscher 2001, S. 65.

379 Ebd., S. 70.
} 
sich die Literatur in der von Wittstock eröffneten Konkurrenzsituation mit den Medien nicht nur behaupten, sondern sie sich diese auch zu Nutze machen konnte, um Breitenwirkung zu sichern. Hielscher macht gar einen 'Boom [...] der deutschen Gegenwartsliteratur' geltend, wegen dem er es als unproduktiv ansieht, die unterschiedlichen ästhetischen Ansätze der jungen Generation gegeneinander oder auch gegenüber denen der älteren auszuspielen. ${ }^{380}$ Zum einen spiegelt sich in Hielschers Aussage die Dynamik des Lesbarkeitsbegriffs, der sich in jedem einzelnen Werk neu konstituieren kann. Zum anderen vermittelt dies, dass Hielscher aufgrund des vorhergehenden Misserfolgs zumindest vorübergehend das Ausbleiben einer kritischen Auseinandersetzung mit den Werken gutheißt.

Hielscher hebt die Bedeutung eines generellen 'Mentalitätswandel[s]' hervor. ${ }^{381}$ Dieser manifestiere sich in der Ablehnung einer elitär didaktischen Nachkriegsliteratur und der Umsetzung von realistischen und an internationale Tendenzen anschließenden Erzählformen, die eine Hinwendung zum Leser bewirkt hätten. ${ }^{382}$ Der Verdienst der 'Neuen Deutschen Lesbarkeit' sei es, 'die Wahrnehmung für solche Texte [geschärft] und ein Klima [geschaffen zu haben], in dem eine junge, erzählerische Literatur Geltung bekam und Verlage bereitwilliger das Risiko eingingen, sie auch zu verlegen. ${ }^{383}$ Hielscher erklärt hier die in Wenn der Kater kommt indizierte Entwicklung hin zu einer Akzeptanz von Lesbarkeit für abgeschlossen. Er betont vor allem die Bedeutung der Marktbedingungen, die deutlich macht, dass die Lesbarkeitsbestrebungen im Kontext der Jahrtausendwende nicht auf rein ästhetischer Ebene umzusetzen gewesen wären, sondern die Prozesse zur Etablierung eines literarischen Programms zusehends komplexer werden.

Einen Erfolg erkennt auch Politycki: 'mit dem Durchbruch der "Neuen Deutschen Lesbarkeit" meldete sich unsere Gegenwartsliteratur zurück unter die europäischen Literaturen.' ${ }^{384}$ Politycki benennt den Erfolg des Lesbarkeitsprogramms ebenso mit dem

\footnotetext{
380 Vgl. ebd., S. 67.

$381 \mathrm{Ebd}$.

382 Vgl. ebd., S. 67. Gegen Ende des Essays liefert Hielscher einige konkrete Beispiele: 'Und wenn man an die Erfolge ganz junger Autoren denkt wie Benjamin Lebert, Benjamin von Stuckrad-Barre, Alexa Hennig von Lange oder immer wieder Christian Kracht, muss man bedenken, dass die Leser dieser Bücher begeistert waren, überhaupt Autoren entdecken zu können, die über das schrieben, was ihre Welt war, mit ihrer Sprache und über ihre Empfindungen. Dass hier ein junges Publikum Autoren wie Popstars behandelt und verehrt, ja anhimmelt, darüber mag man kulturkonservativ die Nase rümpfen, in Wirklichkeit ist es für die Literatur als Identifikationsmittel, für das Lesen und die Bücher, und damit auch für die Verlage und den Buchhandel ein absolut positives Signal.'; ebd., S. 70f.

383 Ebd., S. 70.

384 Politycki 1998 a, S. 5.
} 
Erreichen von Breitenwirkung, das er zudem um eine internationale Komponente erweitert. Allerdings steht er dem erlangten Erfolg der deutschen Literatur skeptisch gegenüber und zweifelt in seinem Artikel 'Der amerikanische Holzweg' (2000) aufgrund des zunehmenden amerikanischen Einflusses dessen Fortbestehen an:

Geht's ihr nicht gut, der allerneuesten deutschen Literatur, jetzt, wo wir vor lauter Frolleinwunder, Neuer Deutscher Lesbarkeit, Popliteratur und Renaissance des Erzählens gar nicht wissen, wie wir noch hinterherkommen sollen mit dem Lesen? Oh ja, es geht ihr wieder gut - fragt sich nur, wie lange noch [...]. ${ }^{385}$

Politycki impliziert den Wunsch nach nachhaltigem Erfolg, was in Bezug auf die Tradition als weitere Strategie gewertet werden kann, Lesbarkeit als qualitativ hochwertige poetologische Kategorie zu etablieren. Während er den unmittelbaren Einfluss des Programms der 'Neuen Deutschen Lesbarkeit' in Form von Verkaufserfolg und Popularität begrüßt, zweifelt er jedoch an der Qualität der praktischen Umsetzung des von ihm vertretenen Literaturprogramms. Dies macht er daran fest, dass sich die deutschen Autoren bei der realistischen Abbildung der Wirklichkeit einer Sprache bedienten, die durch die amerikanischen Vorbilder gefiltert worden sei: '[Wir erhalten] diese Wirklichkeit in immer stärkerem Umfang aus zweiter Hand, aus der Hand dessen nämlich, der sie für uns vorformuliert und geprägt hat - während wir selbst [...] über kurz oder lang zum Hinterwäldler herunterkommen.' 386 Politycki plädiert für einen differenzierten Umgang mit den englischsprachigen Vorbildern: er fordert mehr Originalität und weniger intertextuelle Orientierung, ein neues Selbstbewusstsein der deutschen Literatur, die sich nach dem Anschluss an internationale Tendenzen nun von den Vorbildern emanzipieren soll, um nachhaltig ihre Qualität sowie ihr Renommee und ihren Erfolg zu sichern.

Eine weitere Strategie, um die Nachhaltigkeit des Einflusses von Lesbarkeit zu sichern, besteht für Politycki in der Einleitung $\mathrm{zu}$ der Farbe der Vokale darin, lesbarkeitskritische und -oppositionelle Vertreter des deutschen Literaturbetriebs für Lesbarkeit $\mathrm{zu}$ gewinnen. ${ }^{387}$ Als Beispiel für eine poetologisch paradigmatische Verschiebung hin zur Lesbarkeit kann Greiners Essay Ulrich Greiners Leseverführer.

\footnotetext{
385 Politycki 2000.

386 Ebd.

387 Vgl. Politycki 1998 f, S. 9.
} 
Eine Gebrauchsanweisung zum Lesen schöner Literatur (2005) gelten. Greiner, im Literaturstreit ein Hauptvertreter der 'Neuen Moderne', spielt bereits im Titel auf Wittstocks 'Prinzip der Verführung' an. Ebenso impliziert der Terminus das beispielsweise von Gutzkow bekannte Prinzip, den Leser sicher durch den Text geleiten zu wollen, um dadurch dessen Lesbarkeit sicherzustellen.

Zudem spricht auch Greiners Adressierung für Lesbarkeit. Er wendet sich in seinem Buch an Leser, die lesen, 'weil sie gerne lesen': 'Für Sie also habe ich dieses Buch geschrieben - nicht für den Kenner und nicht für den Profi, nicht für den Germanisten und nicht für den Bibliothekar. ${ }^{388}$ Greiners Buch richtet sich in der Terminologie Wittstocks bzw. Johnsons und Woolfs an den 'common reader', den er den professionellen Lesern vorzieht. Dadurch entdeckt Greiner die breite Masse als Publikum und wertet sie als Adressat auf. Dementsprechend rücken in seinen theoretischen Ausführungen Lesbarkeit und ihr qualitativer Wert in den Fokus:

Wahr ist immerhin, dass es leichte Texte und schwierige gibt [...]. Die Qualität der Werke [hat] mit ihrer Schwierigkeit wenig zu tun. Es ist nicht so, dass die schwierigeren Bücher immer die besseren sind und die leichteren die weniger guten. Joseph Roths "Hiob" zum Beispiel ist ein außerordentlich leicht lesbarer Roman und zugleich ein großes Kunstwerk, [...]. Oder [...]: Ich halte den 1250 Seiten umfassenden gelobten Roman "Die Fälschung der Welt" von William Gaddis (deutsch 1998) nicht nur für eine schwer lesbare Demonstration stupender Gebildetheit, sondern außerdem für missraten. ${ }^{389}$

Greiner dekonstruiert - ohne näher auf deren Vorhandensein im deutschen Literaturbetrieb einzugehen - die Annahme, dass nur komplexe Texte ästhetisch wertvoll seien. Dies erreicht er einerseits durch die Assoziation von leichter Lesbarkeit mit dem Prädikat 'Kunst', das er zudem durch die Größenmetapher intensiviert. Andererseits wählt er ebenso wie Franzen die Werke William Gaddis' als Beispiel für Komplexität. Greiner sieht dessen Bildung zwar als Qualitätsparameter an, disqualifiziert jedoch deren literarische Einarbeitung aufgrund der schweren Lesbarkeit.

Greiner beendet seinen Beitrag mit einer Kanondiskussion, bei der er dem 'common reader' denselben Einfluss einräumt wie den Forschern:

Es gibt den Höhenkamm der kanonischen Texte, was Sie allein schon daran erkennen können, dass sich die Schriftsteller immer wieder und immer von neuem

\footnotetext{
388 Greiner 2007, S. 9f.

389 Ebd., S. 199f.
} 
darauf beziehen. Aber dieser Höhenkamm ist nicht unveränderlich - wie auch das reale Gebirge vulkanischen Neubildungen und Erosionen unterliegt. ${ }^{390}$

Greiner zementiert die Bedeutung von traditionellen, dichotomen Modellen als Orientierungsgrößen zur Qualitätsdiskussion. Allerdings sieht Greiner die qualitativ hochwertige Position nicht als hermetisch abgeriegelte Kategorie an, sodass Reevaluierungen durch dynamische Prozesse jederzeit möglich sind. In Bezug auf Lesbarkeit bedeutet dies, dass der Aktivismus der 1990er Jahre durch die Aufwertung einer breiten Leserschaft und durch das Werben um Autoren, Verlage, Kritiker und Germanisten von Lesbarkeit inspirierten Werken Zugang zum 'Höhenkamm' eröffnet hat. 


\section{Kapitel 4: Helmut Krausser, Daniel Kehlmann, Thomas Glavinic und der Kampf für die 'gute Sache'}

Im Text+Kritik-Band zum Werk Daniel Kehlmanns (2008) verwendet Helmut Krausser den Begriff einer 'guten Sache'. 391 'Gut' hat in der Diskussion um die öffentliche Funktion der deutschen Literatur eine bedeutende Tradition. Anders etwa als in Thomas Manns Ansprache im Goethejahr, in der Mann sowohl moralische als auch ästhetische Qualität einfordert, beinhaltet 'gut' bei Krausser keine moralische Botschaft, sondern steht für ein poetologisches Programm, das sich der Lesbarkeit verschreibt. Mit diesem Programm vertritt Krausser im Tagebuch des Juli 1994 den Anspruch, ein 'verbindendes Qualitätsparadigma [...] auf das sich alle [...] einigen können' zu etablieren. ${ }^{392}$ Die poetologischen Parameter dieses Programms und dessen praktische Umsetzung sowie die im Kampf um dessen Etablierung herangezogenen Strategien sollen im Folgenden eruiert werden. Zunächst wird die Rezeption Kraussers in den 1990er Jahren im Fokus stehen, die den Einfluss der Lesbarkeitsdebatte spiegelt, ehe Kraussers und Kehlmanns Zusammenarbeit sowie deren Rezeption durch die Kritik in die Kollaboration von Kehlmann und Thomas Glavinic mündet. Im Anschluss werden die poetologischen und fiktionalen Werke der Autoren aufzeigen, dass das Eintreten für die Lesbarkeit seit dem Literaturstreit $\mathrm{zu}$ tiefgreifenden poetologischen Entwicklungen in der deutschen Literatur geführt hat, die, von den Autoren vorangetrieben, inzwischen auch von der Kritik befürwortet werden.

Die Kritik bespricht Kraussers Romane in den 1990ern explizit im Kontext der vom Literaturstreit angestoßenen Entwicklung, wobei sich eine Unsicherheit hinsichtlich der qualitativen Einordnung von Lesbarkeit abzeichnet. Maxim Biller sorgt sich in seiner Kritik zu Kraussers Fette Welt (1992) um die Zukunft der deutschen Literatur und sieht Krausser als Hoffnungsträger:

Die Krise der deutschen Literatur - das ist also kein sinnlos dahingemurmeltes Mantra auf den Lippen alter und junger Großkritiker. Das ist eine Tatsache. Und Tatsache ist aber auch, daß der junge Schriftsteller Helmut Krausser mit seinem Roman 'Fette Welt' [...] beweist, daß alles [...] zwar die Wahrheit ist und trotzdem nicht richtig. [...] Denn der 27jährige Krausser hat [...] ein richtiges kleines Meisterwerk hingelegt [...] und gezeigt, dass er über das Talent eines echten

\footnotetext{
391 Krausser 2008, S. 57.
}

392 Krausser 1997, S. 21. 
Erzählers verfügt. [...] Und vielleicht ist Helmut Krausser der erste Großmeister dieser kommenden Zeit. Es wäre ihm und uns wirklich zu wünschen. Vor allem aber unserer Literatur. ${ }^{393}$

Die negative Einstellung gegenüber der deutschen Literatur der Zeit spiegelt die Ausgangslage der Suche nach ästhetischen Lösungsansätzen im Literaturstreit. Biller, der selbst Autor und Kritiker ist, nimmt die Kritik in Schutz und macht die Autoren für den Misserfolg der deutschen Literatur verantwortlich. ${ }^{394}$ Ebenso sieht er die Autoren in der Pflicht, diesen Missstand zu beheben, wobei er sowohl Krausser als auch dem Erzählen eine zentrale Bedeutung zumisst, die Kraussers poetologisches und fiktionales Werk und dessen Rezeption als Indikator für den Erfolg des poetologischen Prinzips der Lesbarkeit etabliert. Die Fokussierung auf eine Person erscheint jedoch als nicht nachvollziehbarer Anspruch, der von einem Autor nicht zu erfüllen ist und der Dynamik und Vielfalt des Lesbarkeitsbegriffs unterläuft.

Auch Christa Fenzl diskutiert in ihrer Rezension zu Fette Welt eine mögliche Vorreiterrolle Kraussers: 'Ist das, was Helmut Krausser [...] in der Sprache von hier und heute erzählt, eine ganz neue Komponente und hervorstechend auf dem Literaturmarkt? Was steckt hinter seiner ungemein frischen, spontanen, mitreißenden Formulierungskunst? ${ }^{395}$ Fenzl benennt die realistisch an die damalige Alltagssprache angelehnte Stilistik als das originelle Qualitätsmerkmal des Romans. Dass diese Ansicht umstritten war, zeigt Lutz Hagestedts Kritik in der Süddeutschen Zeitung: Krausser habe 'einen leichten Ton gefunden, aber nicht immer gelingt ihm die Wanderung auf dem schmalen Grat zur Trivialität.' ${ }^{396}$ Hagestedt disqualifiziert einen leicht verständlichen Stil zwar nicht generell, hegt jedoch qualitative Zweifel. Dies spiegelt Auerbachs Ergebnis, dass der Realismus in der Literaturgeschichte zuweilen als Kategorie angesehen wurde, die nicht mit dem hohen Stil zu vereinbaren ist. ${ }^{397}$ Die Rezeption von Fette Welt positioniert ein leichtes Textverständnis demnach als umstrittene Kategorie im Spannungsfeld qualitativer Hierarchien.

\footnotetext{
393 Biller 1992.

394 Krausser hingegen kritisiert das Feuilleton dafür, die Literatur durch die Privilegierung einer 'Gesinnungsästhetik' ästhetisch und thematisch gehemmt sowie gut verkauften und beim Publikum beliebten Büchern den Wert abgesprochen zu haben; vgl. Krausser 1995, S. 95-97.

395 Fenzl 1993.

396 Hagestedt 1992.

397 Vgl. Auerbach 1977, S. 515f.
} 
Kraussers Roman Melodien (1993) attestieren die Kritiker eine schwere Lesbarkeit. ${ }^{398}$ Eine genaue Analyse zeigt jedoch, dass die Komplexität hauptsächlich im intellektuellen Stoff gesehen wurde, wohingegen die ästhetischen Qualitäten als lesbarkeitsfördernd eingestuft wurden, sodass sich eine ausgleichende Tendenz abzeichnet. Verena Auffermann schreibt, dass es Krausser gelungen sei, schwere thematische Sachverhalte in 'gaumengerechte, um nicht zu sagen, leichtverdauliche Façon zu bringen.' 399 Affermanns Verzehrmetapher spricht für einen thematisch diffizilen Text, der auf eine Weise gestaltet wurde, die dem Leser eine leichte Lektüre ermöglicht. Herbert Heinzelmann greift auf ein Bild aus der griechischen Mythologie zurück: 'Ein Labyrinth? Wohl eher die literarische Behauptung eines Labyrinths. Der Leser wird vom Autor recht sicher am Ariadnefaden gehalten. ${ }^{400}$ Heinzelmann macht eine grundsätzliche Komplexität geltend, die Krausser dadurch umgeht, dass er den Leser sicher durch die schwierigen Passagen des Romans führt, was an die Autorschaftskonzeptionen Gutzkows und Greiners erinnert. Jörg Bartels Besprechung des Romans rekurriert auf Höhenmetaphorik: 'Krausser erzählt das spielend leicht, [...] wechselt immer wieder vom hohen in den Schnodderton.' ${ }^{401}$ Ein solches Changieren zwischen dem hohen und niederen Stil steht für eine Tendenz zur Mitte, die nicht durch eine stilistische Mischform, sondern durch das Wechselspiel Lesbarkeit garantiert - eine ähnliche Strategie, wie sie Wieland bei der Konzeption des Teutschen Merkur verfolgt. Lutz Hagestedt führt darüber hinaus eine weitere rhetorische Kategorie an, indem er die Wirkungsabsicht der Melodien hervorhebt: 'Docere et delectare, belehren und unterhalten, heißt die Devise dieses großen Romans. ${ }^{402}$ Hagestedt knüpft die horazische Kombination von Unterhaltung und Didaktik, wie sie auch bei Wieland und Keil erscheint, an eine Größenmetaphorik, mit der er dem Roman Qualität zuspricht. Unterhaltung fungiert nicht als eigenständiges Qualitätsmerkmal.

Die Rezeption von Kraussers Roman Thanatos (1996) kann als Barometer für einen poetologischen Umschwung gelesen werden, im Zuge dessen zentrale Aspekte des Lesbarkeitsbegriffs zum Desiderat wurden. Tilmann Krause konstatiert im Tagesspiegel:

\footnotetext{
398 Joachim Türk etwa spricht von 'Lesemühe'; vgl. Türk 1993.

399 Auffermann 1993.

400 Heinzelmann 1993.

401 Bartel 1993.

402 Hagestedt 1993.
} 
Light soll man heut' sein. Nur nichts Schweres, nur nichts Belastendes. Auch kurz hat man's gern und mit Pfiff. Ein Spielverderber, wer das Arge nur denkt. [...] Helmut Krausser, soviel steht fest, mag das Weich- und Weggespüle nicht. [...] Als alle sich zu freuen begannen, daß endlich auch die lieben Deutschen jene kleinen gesitteten Geschichtchen erfinden, die man jetzt überall lesen kann, schleuderte der wilde Mann aus München ein Mammut-Epos über die Faszination der Musik in die Menge. [...] Mit seinem neuen 500-Seiten Roman 'Thanatos' wirft uns der 32jährige einen weiteren Knüppel zwischen die Beine. ${ }^{403}$

Krause liefert ein Indiz für den Erfolg des Lesbarkeitsaktivismus, in dessen Kontext viele positiv aufgenommene Romane entstanden waren. Dabei verweist er auf einen verspäteten Anschluss an internationale Tendenzen in Deutschland. Die Wirkung benennt er damit, dass Autoren den Lesern die Lektüre so leicht als möglich gestalten wollen, wobei er Komplexität und Länge als zentrale Kategorien für Schwierigkeit anführt. Aufgrund dieser beiden Aspekte setzt er Kraussers Romane Melodien und Thanatos in Opposition zu den Vereinfachungstendenzen. Krause bestätigt demnach den frühen Erfolg des poetologischen Umschwungs zur Lesbarkeit, wobei er diesem jedoch kritisch gegenübersteht und Krausser deswegen qualitativ hervorhebt, weil er sich diesem vermeintlich nicht anschließt.

Die Komplexität der Romane sieht Ulrich Baron als Grund für deren geringen Verkaufserfolg:

Bei gefälliger Aufbereitung hätte 'Thanatos' ein weiteres umsatzträchtiges Stückchen Genieersatz-Literatur werden können. Aber Helmut Krausser will nicht nur mehr, er kann auch mehr als etwa ein Patrick Süskind oder Robert Schneider. Statt Ambition ist hier eine Begabung am Werk, die allen intellektuellen Tonfallschwindel in wohlkalkulierter Figurenrede denunziert. ${ }^{404}$

Baron verweist auf die Bestsellerautoren von Das Parfum und Schlafes Bruder, die im Hinblick auf die Genie-Thematik ähnliche Romane wie Krausser geliefert hatten. Adornos und Horkheimers Einfluss spiegelt sich darin, dass Baron die kommerziell erfolgreicheren Werke wegen ihrer vermeintlich niedrigeren ästhetischen Qualität Kraussers Thanatos unterordnet. Die finanzielle Unterlegenheit führt Baron auf die höhere Qualität zurück, die ihm zufolge mit Breitenwirkung nicht kompatibel ist. Es finden sich klare Züge des Idealismus, da Kunst und Genie hier keinen Erfolg bezwecken dürfen.

\footnotetext{
403 Krause 1996.
}

404 Baron 1996. 
Günter Franzens Thanatos-Kritik in der Zeit deutet darauf hin, dass die Kritik Lesbarkeit zusehends als anzustrebendes Ideal ansieht:

Wenn es einem jungen, ästhetisch ambitionierten Vertreter der wegen ihrer Leserunfreundlichkeit und mangelnden Welthaltigkeit vielgescholtenen deutschen Literatur denn wirklich einmal gelingt, aus dem Gatter der autobiographisch oder politisch motivierten Bekenntnisprosa auszubrechen und draufloszufabulieren, was Mundwerk und Software hergeben, kennt die Dankbarkeit der darbenden Kritik keine Grenzen. ${ }^{405}$

Franzen rekurriert auf den Topos der Unzufriedenheit der deutschen Kritik mit der einheimischen Literatur, die er an einer schweren Lesbarkeit und einem Mangel an phantasievoller Darstellung festmacht. Außerdem erkennt er die Ablehnung einer politisch didaktischen Dimension. Franzens Beobachtungen sprechen für eine frühe Wirksamkeit der 'Neuen Deutschen Lesbarkeit' auf die Kritik, die sich eine leichte Zugänglichkeit bei gleichzeitig hohem ästhetischen Anspruch und eine sowohl thematische als auch sprachliche Wiederbelebung des Realismus zum Leitbild wählt.

Noch deutlicher tritt der Einfluss der 'Neuen Deutschen Lesbarkeit' auf den deutschen Literaturbetrieb bei der kontroversen Rezeption von Kraussers Roman Der große Bagarozy (1997) hervor. Thomas E. Schmidt setzt Kraussers Roman in der Frankfurter Rundschau explizit in deren Kontext:

\begin{abstract}
Man gewinnt den Eindruck, daß dieser Roman vor allem deswegen geschrieben wurde, weil sich ein Autor entschloß, auf die Diskussionen über Unterhaltsamkeit und Intensität deutscher Gegenwartsliteratur vorauseilend zu antworten: Er hat sich einen spektakulären Stoff gewählt und alles, was an eine komplexe Erzählweise erinnert, vermieden. Statt dessen: kurze Kapitel, einfacher Stil, für gelehrte Leser einige nicht zu schwer entschlüsselbare literarische Anspielungen, vor allem auf Thomas Mann. Diese Literatur schielt am Leser vorbei auf zukünftige Lizenzverkäufe. ${ }^{406}$
\end{abstract}

Schmidt führt Themenwahl, Kürze, eine leicht zugängliche Sprache und intertextuelle Referenzen als Garanten für finanziellen Erfolg an. Diese Kategorien setzt er korrekterweise zum Programm der 'Neuen Deutschen Lesbarkeit' in Bezug. Erstere dienen der Gewinnung einer breiten Leserschaft, wohingegen die postmoderne Doppelkodierung auch auf ein elitäres Publikum zielt. Dabei fungiert die Moderne als

\footnotetext{
405 Franzen G. 1996.
}

406 Schmidt 1997. 
Referenzpunkt. Schmidt bestätigt die angeführten Kategorien als valide Mittel zur Breitenwirkung, lanciert jedoch zugleich eine Kritik, indem er ein solches Literaturprogramm im Sinne der 'Kulturindustrie' ablehnt. 407

Stephan Wackwitz hingegen bewertet in Die Woche, die von 'Uwe Wittstock und seinen Mitstreitern' proklamierte 'Trendwende' als positiv, wobei er Krausser als Indiz für deren Vollzug anführt:

Die handwerklich und intellektuell anspruchsvolle, dabei auch unterhaltende deutschsprachige Literatur jedenfalls: Es gibt sie längst. Genau die Leute, von denen sie ein bisschen wohlverstandenen Lobbyismus verdient hätte, werden sie, statt genau hinzusehen, oft genug wieder einmal pauschal totsagen. Wir sollten damit aufhören. ${ }^{408}$

Wackwitz sieht Lesbarkeit als positive Entwicklung für die deutsche Literatur. Die qualitative Ambition macht er an einer hochwertigen Umsetzung einer intellektuell ansprechenden Thematik fest. Während Wackwitz die Verschmelzung von Unterhaltung und Ambition positiv rezipiert, sieht er seine Kritikerkollegen in der Bringschuld, aktiv für die deutschsprachige Gegenwartsliteratur zu werben. Den Autoren weist er eine Vorreiterrolle bei der Einleitung des Umschwungs zu, den die Kritik noch zu vollziehen hat.

Krausser teilt im Tagebuch des Oktober 1997 Wackwitz' Kritik am Feuilleton und nimmt die Autoren in Schutz:

$\mathrm{Da} ß$ Kritiker sich eine ärmliche Literaturgegenwart herbeiphantasieren, um selbst, in ihrer betrauernswerten Sekundärexistenz, eine beachtete Rolle zu spielen, ist ekelhaft. Die deutschsprachige Literatur ist, wie es für eine der führenden Industrienationen mit bewegter Vergangenheit ja auch wahrscheinlich sein müßte, eine blühende, fruchtbare, vielfältige. ${ }^{409}$

Krausser wirft der Kritik vor, eine Krise der deutschen Literatur herbeizuschreiben, um sich selbst $\mathrm{zu}$ profilieren. Als Grundvoraussetzung für eine florierende Literaturlandschaft etabliert er die finanzielle Prosperität, die literarische Qualität

\footnotetext{
407 Ähnlich argumentiert Martin Krumbholz: 'Dies ist nur eine schöne, witzige, beinahe perfekt erzählte und höchst kurzweilige Geschichte. Thanatos light, sozusagen. Und hat, demnach, das Zeug zum Bestseller.' (Krumbholz 1997). Durch das 'nur' ordnet Krumbholz den leichter zugänglichen und unterhaltsameren Bagarozy dem ästhetisch und thematisch komplexeren Thanatos qualitativ unter.

408 Vgl. Wackwitz 1997.

${ }^{409}$ Krausser 2000, S. 19.
} 
sichert. Ein ähnlicher Aspekt findet sich beispielsweise auch in Wielands Einführung in den Teutschen Merkur. Die Betonung der Geschichte weist auf einen reichen Fundus hin, der den Autoren zur Verfügung steht. Krausser sieht Deutschland angesichts seines Wohlstandes als fruchtbaren Nährboden für eine erfolgreiche Literatur an und vertritt wie Wackwitz die Auffassung, dass sich die Kritik noch nicht auf die Strömungen der Gegenwartsliteratur eingelassen habe.

Im Tagebuch des Juni 1993 macht Krausser den Reflex der Kritik, der deutschen Gegenwartsliteratur eine Krise einzureden, an einem mangelnden Selbstbewusstsein im globalen Kontext fest:

Erst war ich der deutsche Bukowski, dann der deutsche Eco, [...] das ist schon ziemlich witzig. Die Deutschen können sich noch nicht daran gewöhnen, daß ich einer der ihren bin. Bezeichnend für den hiesigen Minderwertigkeitskomplex: Ein halbwegs erfolgreicher Autor hierzulande wird dem Publikum immer als 'der deutsche XY' verkauft. ${ }^{410}$

Krausser fordert die deutsche Literaturszene auf, selbstbewusst gegenüber den marktdominanten internationalen Autoren aufzutreten. Er macht geltend, dass er wegen seines literarischen Programms nicht in das zur Mitte der 1990er Jahre vorherrschende Bild der deutschen Literatur passt. Deswegen werden Parallelen zu Vorbildern aus dem Ausland gezogen, anstatt eine auf Unterhaltung und Qualität zielende Literatur, wie sie Krausser anstrebt, als Teil der Literaturszene anzuerkennen.

Krausser differenziert, denn wie Politycki hält er die erfolgreichen Strömungen der 'Popliteratur' und des 'Fräuleinwunders' nicht für eine adäquate Umsetzung der Forderung nach einer unterhaltsamen und ambitionierten Literatur und kritisiert exemplarisch im Dezember 1999:

Judith Herrmann - das Ende von Etwas. [...] Die Kritiker haben [...] jahrzehntelang gelogen, [...] bis alle Qualitätsparameter verbraucht waren, bis der Verkaufserfolg als letzter übrig blieb. Seither übernahm das Marketing den Laden. ${ }^{411}$

Krausser attackiert eine von der Kritik durch vermeintliche Fehleinschätzungen verschuldete Qualitätsminderung zugunsten einer Kommerzialisierung von Literatur, die

\footnotetext{
410 Krausser 1995, S. 246.

${ }^{411}$ Krausser 2000, S. 325 bzw. $380 f$.
} 
letztlich den finanziellen Erfolg als einzigen Bewertungsparameter übrig ließ. Deswegen fordert er eine Ablösung der Popliteratur:

Kampfansage gegen die Spaßkultur, gegen Kunst, deren Warenwert sich über Geld definiert. [...] Bildung und Intelligenz als oberste Werte. Natürlich ist Kunst Unterhaltung, aber es gibt Unterhaltung auf diversem Niveau. [...]

Den absurden und wichtigtuerischen Pop-Diskurs lächerlich machen, wo immer möglich. ${ }^{412}$

Krausser lehnt kommerziellen Erfolg nicht generell ab, kritisiert aber die Nichtbeachtung ästhetischer Qualitäten zugunsten des Verkaufsergebnisses. Er führt Unterhaltung implizit als Grundvoraussetzung für hohe Absatzzahlen an. Krausser geht nicht von einem exkludierenden Verhältnis zwischen Unterhaltung und Kunst aus, sondern entwirft wie Wittstock eine Rangkontroverse zwischen verschiedenen Unterhaltungsbegriffen. Er bezeichnet Unterhaltung als elementaren Bestandteil der Kunst, fordert jedoch Qualitätsstandards ein, die er über ein intelligentes, von Bildungsgut angereichertes Unterhaltungsprogramm definiert.

Von dieser ambitionierten Unterhaltungsliteratur erhofft sich Krausser, dass sie die 'Popliteratur' langfristig in der Gunst der Leserschaft ablöst:

Das zu Ende gehende Popzeitalter produziert stündlich Wegwerfgötter, von mehr oder minder absehbarer Haltbarkeit, eine Art ideelles Pendant zur Fabrikfertigung [...]. Nun tendiert man wieder zur Größe, zur 'echten' Größe, was die Forderung nach einem komplexen, sich der schnellen Kommerzialisierung verweigernden Werk beinhaltet.

'Echter' und 'größer' ist also, was länger hält, länger unterhält. ${ }^{413}$

Den Werken der Popkultur attestiert Krausser nur eine geringe Lebensdauer. Dem setzt er komplexere Werke entgegen, die sich auf die Dauer durchsetzen würden. Die an Horkheimer und Adorno erinnernde Fabrikmetapher fungiert als Ausdruck davon, dass sich auf lange Sicht komplexere Formen durchsetzen, da sie länger und auf hochwertigere Weise unterhalten. Zur Benennung von Qualität rekurriert Krausser auf den Begriff des 'Echten', der an Bölls Ausführungen erinnert, sowie auf Größenmetaphorik. Inwieweit eine solche Theorie aufrechtzuerhalten ist, kann etwa anhand von Christian Krachts Poproman Faserland (1995), der einen festen Platz im

\footnotetext{
412 Krausser 1997, S. 148.
}

413 Ebd., S. 20. 
Gegenwartskanon hat, hinterfragt werden: schneller und nachhaltiger Erfolg schließen sich demnach nicht zwingend aus. Ebenso fragwürdig ist es, unmittelbar erfolgreichen Werken die Größe abzusprechen, zumal dies Kraussers eigener Wertschätzung für Kehlmanns Vermessung widerspricht.

Die Entwicklung einer ambitionierten Unterhaltungsliteratur sieht Krausser im Tagebuch des September 1996 erst am Anfang:

Zur Zeit geht die Vorbereitung einer Ära vor sich [...]. Aber vielleicht sind die Zeichen dessen wirklich nur sichtbar für jene, die aktiv mitgestalten. [...] Man ahnt, wie sie aussehen könnte, aber die Ahnung wird erst Wort im Moment, da irgendwer die Tore öffnet. ${ }^{414}$

Krausser erkennt eine Umbruchphase in der deutschen Literatur. Er hebt die Bedeutung des Aktivismus von Autoren hervor, wobei der von ihm gezeichnete Prozess im Wesentlichen Kuhns Theorie der revolutionären Krisenzeit und des von einer kleinen Gruppe initiierten Paradigmenwechsels entspricht. Gegen Ende der 1990er Jahre zeichnet sich in Kraussers Tagebucheinträgen die Tendenz zur Lesbarkeit ab. Beispielsweise kritisiert er im Oktober 1997 Raol Schrotts Roman Finis Terrae (1995): 'Könnte leserfreundlicher sein, ohne an Poesie und Kunstfertigkeit zu verlieren'. ${ }^{415}$ Künstlerische Qualität ist Desiderat, jedoch nicht um den Preis einer diffizilen Lektüre. Diesen Anspruch zementiert Krausser im Februar 2002 im Gespräch mit seinem Kollegen Thomas Hettche:

Die [...] Zeiten sind vorbei, da ich mir für Literatur übertrieben viel Mühe machen möchte. Ich will vom Autor auf einem Surfbrett durch die Wogen getragen werden. [...] Ob das ein schlechtes Zeichen sei? Es ist nun mal, wie es ist. Ich steh dazu. ${ }^{416}$

Krausser plädiert nicht dafür, auf schwierige Passagen gänzlich zu verzichten. Autoren können schwierig zu bezwingende 'Wellen' als Textgrundlage einbauen, müssen dem Leser dann jedoch auch ein Hilfsmittel, das 'Surfbrett', zur Hand geben, um jene meistern zu können. So nimmt Krausser die Autoren in die Pflicht, ihre Werke lesbar zu gestalten, indem sie den Lesern über schwierige Passagen sicher und auch vergnüglich hinweghelfen, was in der Surfmetaphorik angelegt ist. Lesbarkeit erscheint als

\footnotetext{
414 Ebd., S. 333.

415 Krausser 2000, S. 61.

416 Krausser 2003, S. 297.
} 
komplexes Konstrukt. Sie ist zunächst ein Anspruch der Leser an die Autoren, dann die dem Text zugrundeliegende Strategie mit Tendenz zur Mitte und hat schließlich die Funktion, auf den Leser als unterhaltendes und vereinfachendes Mittel zu wirken. Krausser sieht Lesbarkeit als poetologischen Status quo in der deutschen Literatur, den er entgegen möglicher Zweifel an dessen Validität unterstützt.

Den Grund dafür, dass viele Autoren weiterhin ein auf Komplexität gründendes Literaturprogramm verfolgen, sieht Krausser in einer 'Angst vor Verständlichkeit, die sie dummen Rezensenten zuvorkommend als Symptom der Trivialität mißinterpretieren. ${ }^{, 417}$ Autoren sehen Verständlichkeit demnach im Hinblick auf eine Missgunst der Kritik als qualitativ minderwertige poetologische Kategorie an. Um dem entgegenzuwirken, wendet sich Krausser direkt an die Literaten: 'Habt den Mut, für Menschen zu schreiben!'418 Die Kategorie des Mutes verweist auf eine riskante Position, die Nachteile nach sich ziehen könnte. Diese besteht daraus, für ein konkretes Publikum zu schreiben. 'Für' verweist darauf, einen für die Leser leicht verständlichen Text zu kreieren. Zudem kann dies als Aufforderung zu einer realistischen Gestaltungsweise verstanden werden, da ein Identifikationspotenzial impliziert wird, indem das Geschriebene mit dem Erfahrungsbereich des Lesers korrespondiert.

Das Lesbarkeitsdesiderat sieht Krausser vor allem im Werk Kehlmanns umgesetzt. Aus beider Begegnung auf der Frankfurter Buchmesse 2003 entwickelt sich die wohl effektivste Zusammenarbeit zur Etablierung von Lesbarkeit in der deutschen Gegenwartsliteratur. Im Vergleich etwa $\mathrm{zu}$ Romantik und Idealismus ist es bemerkenswert, dass sowohl Theoriebildung als auch Praxis der Lesbarkeit von Autoren vorangetrieben wird und beide Bereiche nicht strikt getrennt sind. Krausser koppelt im Tagebuch des April 2004 die Lesbarkeit bei Kehlmann an Unterhaltung:

Daniel ist ein sagenhaftes Talent. Wieso Talent? Wo er bereits ein großer Autor ist. Weil er, wie ich glaube, noch viel mehr Zukunft hat, als er schon Gegenwart besitzt. Der Kerl ist 28 Jahre alt. Wo stand ich mit 28 ? Nicht annähernd dort, wo er heute steht. Er müßte viel berühmter sein. Aber wenn jemand so früh so groß ist, kann er wahrscheinlich nicht berühmter sein. Da hat die Welt ihre Selbstschutzanlagen vor. Obgleich bei ihm Tiefe und Schönheit mit außergewöhnlicher Lesbarkeit einhergehen, um nicht zu sagen: Unterhaltsamkeit. ${ }^{419}$

\footnotetext{
417 Krausser 2006, S. 89.

418 Ebd.

419 Ebd., S. 202.
} 
Größe fungiert als Qualitätsanzeiger. Generell gestaltet Krausser die gesamte Passage mit Termini, die in der deutschen literarischen Tradition zur Benennung hierarchischer Qualitätsverhältnisse herangezogen wurden. Dabei führt er die traditionell oppositionellen Kategorien 'Tiefe' und 'Schönheit' einerseits sowie 'Lesbarkeit' und 'Unterhaltsamkeit' andererseits an. Die romantische Innerlichkeits- und Tiefenmetaphorik evoziert einen unter der Textoberfläche verborgenen Behälter, der einen literarischen Mehrwert garantieren soll. ${ }^{420}$ Der Begriff 'Schönheit' avancierte mit Immanuel Kants Kritik der Urteilskraft (1790) zu einem zentralen Kriterium. Kant stellt die 'schöne Kunst' den 'angenehmen Künsten' gegenüber, wobei er erstere privilegiert, da sie nicht auf schnelles Vergnügen zielt, sondern auf eine bleibende Wirkung. ${ }^{421}$ Für die Literatur folgert Katrin Kohl in Poetologische Metaphern aus Kants Ausführungen, dass fortan "die "schöne" Literatur als "ernste" Kunst [gilt], die ausschließlich im bleibenden Wert ihre Rechtfertigung findet.' ${ }^{422}$ Es handelt sich demnach um Termini, die der traditionellen Unterscheidung zwischen E- und U-Literatur zuzuordnen sind. In Kehlmanns Werk sieht Krausser Elemente beider Kategorien im postmodernen Sinne vereint. Lesbarkeit ist für Krausser eine eigenständige Qualität aus der U-Literatur, die egalitär zu den traditionell prestigeträchtigeren Elementen der E-Literatur verhandelt wird, wobei er Lesbarkeit nicht automatisch mit Unterhaltung verbindet. Neben dieser Kombinationstendenz spricht Krausser zwei weitere Aspekte an, die für seine Zusammenarbeit mit Kehlmann wichtig sein werden: erstens, die Reaktionen des Literaturbetriebs auf Kehlmann; zweitens, die Inszenierung einer Konstellation, die an das Verhältnis Johannes des Täufers zu Jesus Christus erinnert, indem sich Krausser zum Vorkämpfer für die 'gute Sache' stilisiert, wobei sein Werk sich dann in dem seines jüngeren und von der Kritik weniger kontrovers aufgenommenen Nachfolgers erfüllt.

Krausser und Kehlmann beginnen eine Zusammenarbeit zur nachhaltigen Durchsetzung von Lesbarkeit, im Zuge derer sie sich strategisch um die diversen Beteiligten an Literaturproduktion und -rezeption kümmern, die auch Politycki zu gewinnen gedachte. Es handelt sich um einen Zusammenschluss über zwei Generationen unter Fokussierung auf die öffentlichkeitswirksamen Aspekte des

\footnotetext{
420 Vgl. Kohl 2007 b, u.a. S. 308, 344, 555 und 580.

421 Vgl. Kant 1908, S. 303-306 und 327.

422 Vgl. Kohl 2007 b, S. 344.
} 
auktorialen Schaffens. Als erstes überzeugt Krausser Kehlmann davon, vom Suhrkamp zum Rowohlt Verlag zu wechseln. In Text+Kritik schreibt er: 'Ich habe Daniel Kehlmann überredet, zu Rowohlt $\mathrm{zu}$ wechseln, habe meinem damaligen Verleger Alexander Fest einen Welterfolg vorhergesagt, und wenn meine Prophezeiungen auch selten in Erfüllung gehen, werde ich mit dieser einen immer angeben. ${ }^{423}$ Tatsächlich wurde die Vermessung, Kehlmanns erster bei Rowohlt verlegter Roman, zum internationalen Bestseller. Es lohnt ein Blick auf das Verlagsprogramm. Krausser macht sowohl im Interview ähnlich wie Politycki Suhrkamp für 'die Misere der deutschen Erzählkunst' verantwortlich, da der Verlag seiner Auffassung nach 'antipopulistische Literatur durchgepreßt' habe. ${ }^{424}$ Seine Kritik richtet sich gegen ein leserunfreundliches Literaturprogramm, dem die Agenda Rowohlts entgegensteht: 'Den Kern bildet der Buchverlag, der mit seinem Profil den Anspruch seines Gründers verkörpert: gut lesbare Literatur auf hohem Niveau.' ${ }^{425}$ Dieses Desiderat entspricht sowohl Kraussers Anspruch nach einer Fusion von ästhetischer Qualität und leichter Lesbarkeit als auch dem Programm der 'Neuen Deutschen Lesbarkeit'.

In der Folge beginnt Krausser Kehlmann in journalistischen Veröffentlichungen an ein breitgefächertes Publikum weiterzuempfehlen. Im Mai 2004 sagt er dem Magazin Galore: 'Daniel Kehlmann [...] halte ich für besser als mich, und der ist zwölf Jahre jünger. Ein echtes Genie.' 426 Der Begriff des 'Genies' verweist auf die literarische Tradition und fungiert als Ausdruck der Qualität von Kehlmanns Werk. Während die Genieästhetik aufgrund der gegenüber der Leserschaft exponierten Position des Autors traditionell eher nicht lesbarkeitskompatibel erscheint, geht der Begriff hier auf einen Autor über, der für Lesbarkeit eintritt. Zudem erwähnt Krausser Kehlmann häufig in seinen zwischen 2006 und 2012 im Süddeutschen Zeitung Magazin erschienenen, in Anklang an Heine titulierten Deutschlandreisen ${ }^{427}$ Er bringt Kehlmann und ihre geteilte Präferenz für Lesbarkeit in breitenwirksame Medien ein, die eine vielfältige Leserschaft ansprechen - Galore war Deutschlands größtes Interviewportal, das darauf zielte, möglichst viele Aspekte der Popkultur abzudecken, und das Süddeutsche Zeitung Magazin bedient ein bildungsbürgerliches Publikum.

\footnotetext{
423 Krausser 2008, S. 54.

424 Vgl. Steinert 1994, S. 88.

425 Rowohlt 2014.

426 Großmann 2004.

427 Später in einem Band zusammen mit Kraussers Münchner Poetikvorlesungen erschienen, vgl. Krausser 2014, u.a. S. 16, S. 94, S. 280.
} 
Außerdem zielen Krausser und Kehlmann darauf, Lesbarkeit und Unterhaltung auch im akademischen Betrieb zu etablieren. In seinen 'Pathos und Präzision' betitelten Münchner Poetikvorlesungen (2007) nennt Krausser Kehlmann dezidiert als literarischen Einfluss:

Seither gab es viele Bücher, die mich beeinflußt haben, aber kaum ein Werk, das mich dazu gezwungen hätte, mich komplett damit zu beschäftigen.

Doch. Natürlich. Daniel Kehlmann. [...]

Heute, nachdem die Vermessung der Welt weit über eine Million Exemplare nur in Deutschland verkauft hat, höre ich an jedem zweiten Biertisch Debatten darüber, daß Kehlmann doch nur Unterhaltungsliteratur sein müsse, das sei klar und logisch. Verflucht sei die perverse Zeit, die Menschen derlei Gedankengänge einst als logisch anempfohlen hat, als dialektisch notwendiges Zweifeln. Wohingegen man froh sein müßte, daß endlich wieder einmal etwas an sich viel Logischeres stattgefunden hat, nämlich daß sich ein sehr gutes Buch sehr gut verkauft. ${ }^{428}$

Krausser analysiert das Greifen der im Tagebuch noch angedeuteten 'Selbstschutzanlagen', nämlich den Reflex der Kritik, einem hochwertigen Werk aufgrund von hohen Verkaufszahlen Qualität abzusprechen. Die von Krausser kritisierte Diffamierung von Unterhaltung und die Abneigung gegenüber kommerziell erfolgreichen Werken entspricht dem kulturindustriellen Stigma von Adorno und Horkheimer. Hinsichtlich des finanziellen Erfolges lässt Krausser einen unmittelbaren Zusammenhang zwischen Qualität und Verkaufszahlen nicht gelten, sieht den Erfolg auf dem Absatzmarkt aber als an sich logische Konsequenz für ansprechende Literatur.

Diese Kritik führt Krausser im Text+Kritik-Band zu Kehlmanns Werk weiter aus. Die Konzeption der Reihe ermöglicht es Krausser, sich hybride zwischen den Rollen des Autors, des Kritikers und des Akademikers zu bewegen, worin sich die Komplexität der in der Lesbarkeitsdebatte einflussreichen Stimmen spiegelt. Krausser geht davon aus, dass die Vermessung eine Ausnahme für die Rezeption lesbarer und vielfach gelesener, unterhaltsamer Romane in Deutschland darstellen könnte:

Nun ist es so, dass die jüngste deutsche Literatur in den letzten zwei Jahrzehnten einige Meisterwerke hervorgebracht hat, die vom Feuilleton nicht die nötige Würdigung gefunden haben, um sie sozusagen aufs nächsthöhere Level zu heben, sprich auf internationaler Ebene weiterzuempfehlen. Stattdessen wurden öde und artifizielle Machwerke mit Preisen überhäuft, die kaum ein Publikum hinter sich wussten und über die das Ausland, wenn es sie überhaupt wahrnahm, nur den Kopf 
schütteln konnte. Bei Kehlmann, dachte ich, könnte ein Sonderweg der Rezeption möglich sein, eine Ausnahme. ${ }^{429}$

Krausser eröffnet durch die hierarchische Höhenmetaphorik eine qualitative Wertungsskala für Verkaufsserfolg und Anerkennung beim ausländischen Publikum. Dabei gesteht er den deutschen Feuilletons und Literaturpreisen eine wichtige Rolle bei der Weiterempfehlung zu; das Ziel ist es, den deutschen Literaturmarkt zu transzendieren und Erfolge im Ausland einzufahren. Er vertritt die Auffassung, dass die junge deutsche Gegenwartsliteratur höchsten Ansprüchen genügt und erneuert seinen Angriff auf die Kritik, weiterhin leserunfreundliche und bei den Lesern erfolglose Werke zu fördern, was der deutschen Literatur im Ausland einen schlechten Ruf eingebracht habe.

In diesem Kontext reflektiert Krausser über den internationalen Erfolg der Vermessung. Er macht geltend, dass die Synthese von künstlerischem Anspruch und der Ambition, dem Leser durch eine unbeschwerte Lektüre Freude zu bereiten, für Kehlmanns Erfolg beim ausländischen Publikum und der internationalen Kritik ausschlaggebend sei:

[E]inen solchen Schriftsteller hatte die deutsche Sprache lange nicht hervorgebracht. Höchste Belesenheit, bestechende Intelligenz und dezidierter Formwille gingen einher mit leichter Lesbarkeit, dieser Autor strotzte von Traditionsbewusstsein und Seriosität, war dennoch auf höchstem Niveau unterhaltsam, bot brillante Dialoge auf und schob unter die narrative Oberflächenstruktur unendlich viele Tiefenschichten. ${ }^{430}$

Krausser plädiert für Unterhaltung, wobei er seinen Unterhaltungsbegriff höhenmetaphorisch distinguiert. Vermeintlich wertvolle Romane grenzt er dezidiert ab. Den Unterschied $\mathrm{zu}$ minderwertigerer Unterhaltung macht er an der postmodernen Mixtur aus Aspekten der E- und U-Literatur fest. Krausser zieht erneut eine Tiefenmetaphorik heran, um einen qualitativen Mehrwert auszudrücken, die Oberflächenmetapher fungiert zur Betonung einer leicht zugänglichen und stringenten Handlungsführung. Diese Mehrfachkodierung entspricht den postmodernen Kategorien des exoterischen und esoterischen Vergnügens, mit denen eine breite Leserschaft gewonnen werden soll. Die E-Literatur drückt Krausser durch den Begriff der

\footnotetext{
429 Krausser 2008, S. 54.
}

430 Ebd., S. 55. 
'Seriosität' aus, mit dem er literarische und literaturhistorische Bildung, intellektuelle Kapazität und handwerkliches Vermögen verbindet, durch die eine ästhetisch und thematisch anspruchsvolle Leserschaft gewonnen werden soll. Als Begriff aus der ULiteratur dient Lesbarkeit dazu, ein möglichst breites Lesepublikum anzusprechen. In Kehlmanns Roman sieht Krausser diese Strategie ideal verwirklicht und gesteht dem Autor eine Vorreiterrolle bei deren praktischer Umsetzung zu.

Umso beschämender, fährt Krausser fort, sei der Reflex gewesen, der Vermessung Tiefe abzusprechen, sobald sich das Buch der Million verkaufter Exemplare näherte. ${ }^{431}$ Krausser rekurriert auf den Kontext der 'Kulturindustrie' und dessen Trivialitätsstigma gegenüber finanziell erfolgreicher Literatur, das er ebenso wie Wittstock anficht, um sich aktiv für ein Umdenken des Literaturbetriebs einzusetzen.

Eine Analyse der Rezeption der Vermessung bestätigt Kraussers Beobachtung. Als exemplarisch kann Denis Schecks monatliche Bestsellerkolumne im Tagesspiegel gelten. Im November 2005 wertet Scheck die Vermessung als 'glänzend gebauten Roman' und lobt, dass es Kehlmann gelinge, 'mit überraschend viel Humor vom Preis des Wissens, von zwei Arten des Zugriffs auf die Wirklichkeit und vom Unterschied zwischen Aufklärung und Desillusionierung' zu erzählen. ${ }^{432}$ Scheck schätzt sowohl die Struktur des Romans als auch den Umstand, dass komplexe Sujets humoristisch aufgearbeitet werden, was der Mixtur aus Elementen der E- und U-Literatur entspricht. Mit steigenden Verkaufszahlen thematisiert Scheck im Februar 2006 zum ersten Mal den Erfolg des Romans und lanciert umgehend seine erste Kritik: 'Es ist ein bisschen ein Schweinchen-Schlau-Roman'. ${ }^{433}$ Scheck bewertet die zuvor gelobte Thematik als altklug, womit er die Dekonstruktion des E-Elements der intellektuellen Aspiration initiiert. Im August 2006 verwendet er das Verhältnis zwischen Humor und Sujet, um Kehlmanns Roman die Lorbeeren zu entziehen:

Eine unterhaltsame Sparringrunde aus der deutschen Geistesgeschichte erzählt der junge Wiener Schriftsteller Daniel Kehlmann in seinem scharfsinnig konstruierten und gut geschriebenen Buch [...]. Der Reiz dieses historischen Kuriositätenkabinetts erschließt sich auf Anhieb - nicht aber seine Relevanz. ${ }^{434}$

\footnotetext{
431 Vgl. ebd.

432 Scheck 2005.

433 Scheck 2006 a.

434 Scheck 2006 b.
} 
Handwerkliches Können, Intellekt und Unterhaltung sind für Scheck die Kriterien für den Erfolg der Vermessung. Die Metapher der 'Sparringrunde' - ein lockeres Aufwärmen mit offensichtlichem Reiz aber geringer Bedeutung - verweist auf einen Mangel an Qualitäten jenseits der Unterhaltung, die Scheck nicht als Alleinstellungsmerkmal genügt. Im folgenden Monat ist Unterhaltung die einzige Qualität, die er noch gelten lässt: 'Bloß vermag ich jenseits der Unterhaltsamkeit keinen zwingenden Grund anzugeben, warum man unbedingt einen Blick in dieses wundersam bunte historische Kaleidoskop werfen müsste.' ${ }^{435}$ Scheck etabliert Unterhaltung als valide und akzeptierte Qualität, es verlangt ihn aber nach einem nicht konkreter benannten Mehrwert.

Krausser hingegen bewertet Kehlmanns Verkaufserfolg durchweg positiv und schreibt dem internationalen Erfolgszug der Vermessung in Text + Kritik eine Signalfunktion für die deutsche Gegenwartsliteratur zu:

Seit es Kehlmann gibt, wird deutsche Literatur im Ausland, vor allem im englischsprachigen Ausland, nicht mehr wie syrische Lyrik behandelt. Er schlägt Schneisen in den Dschungel der unverhohlenen Missachtung, um die ihm jeder deutsche Literat dankbar sein muss. Niemand hat diesen Erfolg so sehr verdient. ${ }^{436}$

Kraussers Metaphorik versieht Kehlmann mit einer aktiven Rolle beim Durchbruch der deutschen Gegenwartsliteratur auf dem internationalen Markt. Mit der Vorreiterrolle evoziert Krausser das Bild des 'Türöffners' aus den Tagebüchern und lässt auf den Anbruch der prognostizierten neuen Epoche in der deutschen Literatur schließen. Diese zielt speziell auf die Reputation und den Verkaufserfolg der jungen deutschen Literatur im Ausland.

Auch Wittstock erkennt in der Rezeption von Kehlmanns Werk Anzeichen für einen Umschwung im deutschen Literaturbetrieb, der für den nachhaltigen Erfolg des Lesbarkeitsaktivismus spricht. In seiner Laudatio bei der Verleihung des Kleist-Preises an Kehlmann (2006) entwirft er folgendes Szenario:

\footnotetext{
435 Scheck 2006 c.

436 Krausser 2008, S. 57.
} 
[Keinesfalls durfte sich ein Autor dabei ertappen lassen], mit seinen ästhetischen Strategien das Ziel zu verfolgen, möglichst unterhaltsam, möglichst anziehend und zugänglich, also möglichst publikumswirksam zu schreiben.

Denn der Verdacht, er habe sich von der Unterhaltungsindustrie korrumpieren lassen und stricke munter fort am universalen Verblendungszusammenhang, konnte für seine weitere literarische Karriere tödlich sein. So schnell kam man damals von ästhetischen Fragen zu politischen Antworten. ${ }^{437}$

Wittstock kritisiert die Stigmatisierung von Aspekten des Lesbarkeitsbegriffs durch die Kritik. Mit 'zugänglich' verwendet er die gleiche Terminologie wie Becher. Auffällig ist zudem die Betonung der Wirkung, die als 'Wirkungsästhetik' im Zentrum des Programms der 'Neuen Deutschen Lesbarkeit' steht. Wittstock bringt ferner Verkaufserfolg und einen mit ihm verbundenen Trivialitätsverdacht ins Spiel. Die Termini 'Unterhaltungsindustrie' und 'Verblendungszusammenhang' evozieren Horkheimers und Adornos Funktion der Ablenkung der Gesellschaft von kritischen Fragen nach der nationalsozialistischen Vergangenheit durch Unterhaltung. Wie Krausser erkennt Wittstock die Ablehnung einer publikumswirksamen Gegenwartsliteratur: ästhetische Streitfragen werden zu literaturpolitischen, was darauf verweist, dass Lesbarkeit in der deutschen Literaturlandschaft ein von diversen Instanzen heftig debattiertes Politikum darstellt.

Die öffentliche Resonanz auf Kehlmanns Werk und insbesondere auf die Vermessung macht Wittstock als Indikator für einen Wandel im Literaturbetrieb aus:

In unserer Literaturkritik hat sich während den vergangenen zehn, zwölf Jahren manches verändert, die Einsicht, dass literarisches Heil nicht allein nach dem Katechismus der Moderne zu erlangen ist, sondern auch auf anderen Wegen, wird allmählich immer selbstverständlicher. Denn schließlich kann die Forderung nach permanenter literarischer Innovation heute, in einer Phase rabiater technischökonomischer Innovationsschübe weder große Originalität für sich in Anspruch nehmen, noch birgt sie ein nennenswertes zeitkritisches Potenzial.

Zudem ist es wohl keine Überraschung, wenn eine Literatur, die sich verschärfter medialer Konkurrenz ausgesetzt sieht und händeringend um jeden Leser kämpft, nach ästhetischen Konzepten Ausschau hält, die es nicht als zentrale Aufgabe betrachten, Leser zu enttäuschen. [...] Als ein Indiz dafür, wie gründlich sich das literaturkritische Klima hierzulande verändert hat, darf man auch die Resonanz auf Daniel Kehlmanns jüngsten Roman 'Die Vermessung der Welt' betrachten. ${ }^{438}$

Wittstock bekräftigt seine in Leselust geäußerte Ablehnung komplexer moderner Formen. Er resümiert die im Literaturstreit angestoßenen Entwicklungen und proklamiert den Erfolg des Lesbarkeitsprogramms. Wittstock macht diesen an der 
Ablehnung von avantgardistischen Originalitätszwängen fest. Zudem sieht er eine Abkehr von einer engagierten Literatur. Außerdem eröffnet er wie in Leselust ein Konkurrenzverhältnis zwischen der Literatur und neueren Medien: die Literatur muss ästhetische Ansätze finden, die Lektüre für die Leser angenehm zu gestalten, um sich gegen die Konkurrenz durchsetzen zu können. Kehlmanns Vermessung teilt Wittstock die Funktion eines Indikators $\mathrm{zu}$, der die umfassende Akzeptanz auf Lesbarkeit zielender Werke durch den Literaturbetrieb bestätigt. 439

Die durch den Erfolg der Vermessung erarbeitete Sonderstellung Kehlmanns im deutschen Literaturbetrieb wurde in den Feuilletons ausgiebig diskutiert, was wiederum Schlüsse auf Erfolg und Akzeptanz von Lesbarkeit zulässt. ${ }^{440}$ Rainer Moritz schreibt zum Erscheinen von Kehlmanns Episodenroman Ruhm (2009) in der Stuttgarter Zeitung:

\begin{abstract}
Wann durfte es ein deutscher Schriftsteller erleben, das das Erscheinen seines neuen Werkes am selben Abend in den 'Tagesthemen' und im 'heute Journal' ausführlich gewürdigt wurde? [...] Er absolvierte Großlesungen vor über tausend Besuchern; er lässt sich von dem bislang nicht als Belletristikconnaisseur aufgefallenen Reinhold Beckmann in dessen Talkshow zu mitternächtlicher Stunde loben [...]. Mühelos ist es ihm gelungen, woran sich seine zwanzig Jahre älteren Kollegen wie Georg Klein, Matthias Politycki oder Bodo Kirchhoff vergeblich abarbeiteten: die Dauerbefragten Günter Grass, Hans Magnus Enzensberger und Martin Walser abzulösen und unangefeindete Omnipräsenz in den Medien zu erlangen. Daniel Kehlmanns Auftritte - und nicht zuletzt die Absatzzahlen seiner Bücher - signalisieren einen internationalen Aufbruch für die deutsche Literatur, einen 'change'. ${ }^{441}$
\end{abstract}

Durch die Besprechungen in den beiden prestigeträchtigsten und meist gesehenen Nachrichtensendungen im deutschen Fernsehen und dem Auftritt in einer der beliebtesten Talkshows erreicht Kehlmann ein möglichst breitgefächertes Publikum. Ebenso wie seine Lesungen zeigt dies einerseits, dass er sich der zunehmenden Eventisierung der deutschen Gegenwartsliteratur anschließt, und andererseits, dass Kehlmanns Erfolg eine Plattform für Lesbarkeit geschaffen hat, die ein weitaus größeres Publikum erreicht als Kehlmanns Vorgänger in den 1990ern. ${ }^{442}$ Durch den Vergleich zu den Vorgängergenerationen verdeutlicht Moritz nicht nur den Generationskonflikt in der

\footnotetext{
439 Wittstock kann zu diesem Zeitpunkt noch nichts von den Entwicklungen in der Kritik bei steigenden Verkaufszahlen wissen. Diese scheinen sich jedoch mehr aus der idealistischen Ablehnung finanziell erfolgreicher Romane zu speisen, denn aus einer kritischen Auseinandersetzung mit ästhetischen Kriterien.

${ }^{440}$ Eine wissenschaftliche Analyse von Kehlmanns Status als 'literary celebrity’ liefert Braun 2013.

441 Moritz 2009.

${ }^{442}$ Zur Eventisierung vgl. Kohl 2007 a.
} 
Lesbarkeitsdebatte um 2000, sondern auch die zunehmende Komplexität des literarischen Apparates, in der sich Breitenwirkung nicht mehr einzig über das Werk, sondern über eine Kombination aus fachlichem Können, Kompetenz im Umgang mit den Medien und Erfolg beim Publikum definiert. Kehlmann fungiert als herausragendes Beispiel dafür, dass sich die junge deutsche Literatur in ihrer öffentlichen Wahrnehmung an die internationalen Vorbilder annähert und sich einen Markt im Ausland erschließt.

Die Auslandskritik attestiert Kehlmann ebenfalls eine Vorreiterrolle. Luke Harding betitelt seine Guardian-Kritik zum Erscheinen der englischsprachigen Ausgabe der Vermessung mit 'Unlikely bestseller heralds the return of lightness and humour to German literature'. Harding macht die Kombination von wissenschaftlichem Sujet und ästhetischer Seriosität mit den oppositionellen Komponenten der Leichtigkeit und des Humors als das für die deutsche Gegenwartsliteratur ungewöhnliche Element aus. ${ }^{443}$ Er zeichnet sein Bild der deutschen Nachkriegsliteratur, in das er Kehlmann einordnet: 'The novel marks a change in Germany's post-war literary landscape. For decades German fiction has enjoyed the reputation of being serious [...] and a bit dull. It has, for the most part, been preoccupied with the country's grim past. ${ }^{\prime 44}$ Harding thematisiert den E-Pol und konstatiert, dass die deutsche Nachkriegsliteratur im Ausland aufgrund ihrer Auseinandersetzung mit der Nazizeit den Ruf habe, Ernst und Langeweile zu verkörpern. Unterhaltung ist demnach wegen eines politisch moralischen Debets zu kurz gekommen, worin sich die Inhalte des Literaturstreits spiegeln. Dass das Bild der deutschen Literatur im Ausland von der Nachkriegsgeneration geprägt ist, deutet auf eine Bestätigung von Kraussers Annahme hin, dass die deutsche Kritik die in Folge der Lesbarkeitsdebatte entstandenen Werke nicht erfolgreich an das Ausland weiterempfohlen hat.

Harding folgert: Die Vermessung 'does not feel like a "German" novel'. ${ }^{445} \mathrm{Er}$ bestätigt den internationalen Ruf der deutschen Literatur und wertet Kehlmanns Roman als Anzeichen dafür, dass die deutsche Literatur sich zunehmend an internationalen Vorbildern orientiert, zu leichteren und unterhaltsameren Formen übergeht und dadurch

\footnotetext{
${ }^{443}$ Um dies zu untermauern, zitiert er Felicitas von Lovenberg: 'A lot of German literature has the same reputation as Germany does abroad, Felicitas von Lovenberg, literary editor of the Frankfurter Allgemeine Zeitung said. It's serious. And it's all about history. You feel weighed down by it. And then Kehlmann comes along. He's amazing. He's very serious yet at the same time makes it all look so easy and playful. It's very hard to be witty and funny at the same time without being shallow. He's charming as well.' (Harding 2006).

${ }^{444}$ Ebd.

${ }^{445}$ Ebd.
} 
internationale Breitenwirkung erlangt. Dies stellt eine Umkehrung von Kraussers Kritik dar, wonach er vom Feuilleton häufig als der 'deutsche XY' bezeichnet wurde. Während Krausser dem deutschen Literaturbetrieb ein mangelndes Selbstbewusstsein attestiert, stellt das Nicht-Deutsche bei Harding eine positiv besetzte ästhetische Entwicklung dar, die vom Lesbarkeitsbegriff geprägt ist. Diese führt Harding auf den zunehmenden Einfluss von internationalen Autoren auf die junge deutsche Generation zurück:

Increasingly, it seems, young German writers are no longer looking to Thomas Mann and Grass for inspiration, or studying the theories of Theodor Adorno. Instead, they are looking to Anglo-Saxon fiction and Spanish magic realism. ${ }^{446}$

Die Beispiele machen die Tendenz einer Abkehr von Vorbildern sichtbar, die eine lesbarkeitsantagonistische Literatur vertreten: Mann steht für eine komplexe, ernste Literatur der Moderne, Grass für eine politisch und moralisch engagierte Literatur, Adorno und Horkheimer gegen Unterhaltung und Breitenwirkung. Realismus hingegen spricht für Lesbarkeit und die britische und amerikanische Literatur wurden, eminent durch die postmoderne Theorie Leslie Fiedlers, zum Vorbild der 'Neuen Deutschen Lesbarkeit'. ${ }^{447}$

Kehlmanns Abkehr von Schwere, Ernst und Langeweile bewertet auch die Kritik in anderen Ländern als positive Errungenschaft der jungen deutschen Literatur. In Italien schreibt die Republica, dass Kehlmann für die deutsche Literaturlandschaft viele Sympathien geerntet habe, von der sich die Italiener wegen Günter Grass und dessen Zeitgenossen abgewendet hätte. ${ }^{448}$ Die französische Libération lobt die Vermessung deshalb, weil Kehlmann im 'Vergleich mit der von Böll und Grass beherrschten Nachkriegsliteratur, die von Seriosität und Bedeutungsschwere gezeichnet war, [...] eine unbeschwerte und burleske Literatur [vorlegt]. ${ }^{, 49}$ Die Opposition zwischen E- und ULiteratur drückt sich durch einen Generationenkonflikt aus, wobei die komplexe Nachkriegsliteratur der älteren Generation gegen die leicht zugängliche und unterhaltsame Literatur der jungen Generation um Kehlmann angeführt wird. Besonders prägnant findet sich dies in der niederländischen Berichterstattung. Dort heißt es, dass man sich 'nur zu oft über bemühten Tiefgang, Didaktik und Ethikfimmel [gelangweilt

\footnotetext{
446 Ebd.

447 Als Einflüsse auf Kehlmann benennt Harding neben Borges und Nabokov Zadie Smith und Ian McEwan.

448 Anon. 2007 a.

449 Anon. 2007 c.
} 
habe]'. ${ }^{450}$ Der Hauptkritikpunkt der Rezension ist ein Unterhaltungsdefizit, das auf ein intensives Bemühen um Qualität und eine politisch moralische Funktion zurückgeführt wird. Die Frankfurter Allgemeine Zeitung zieht das Fazit, dass den Rezensenten in den Niederlanden 'die Erleichterung, hier könnte um und hinter Kehlmann eine jüngere Generation geistreicher und amüsanter deutscher Schriftsteller heranwachsen, deutlich anzumerken' gewesen sei. ${ }^{451}$ Die Auslandskritik stützt sich auf ein verallgemeinerndes, auf die Generation Grass fokussiertes Bild der deutschen Gegenwartsliteratur, das im Wesentlichen der vom Programm der 'Neuen Deutschen Lesbarkeit' abgelehnten Nachkriegsliteratur entspricht. Aufgrund ihrer eigenen Abneigung gegenüber einer derartigen Literatur begrüßt sie den Umschwung hin zu Lesbarkeit und Unterhaltung. Kehlmann stilisiert die Auslandskritik wohl auch aufgrund seines Erfolgs zur Galionsfigur dieses Wandels, die die poetologischen Kriterien erfüllt, um international Anschluss zu finden.

Im deutschsprachigen Raum hingegen wurde die Stilisierung Kehlmanns zur Leitfigur wegen der Ausrichtung auf Lesbarkeit auch negativ rezipiert. Evelyne PoltHeinzl benennt in der österreichischen Furche folgende Gründe für Kehlmanns Publikumserfolg: 'Seine Romane sind einlässig geschrieben und nicht zu umfangreich, sie versprechen eine philosophische Tiefendimension, ohne $\mathrm{zu}$ verstören. Das prädestiniert sie für das Lizenzgeschäft - "Exportable, Less Weighty German Novels",.452 Die Kritikerin bewegt sich im semantischen Feld von Bechers 'Zugänglichkeit', die dem Leser eine leichte Lektüre garantiert. Ein geringer Umfang verweist auf das rhetorische Prinzip der brevitas. Polt-Heinzl stellt die e-literarische Tiefenmetapher einer für den Leser angenehmen Textgestaltung gegenüber, den Mehrwert bezieht sie auf ein philosophisches Erkenntnispotenzial; bei Kehlmann sieht sie eine aus der Fusion der Qualitäten der E- und U-Literatur resultierende Tendenz zur Mitte. Außerdem stellt Polt-Heinzl die ästhetische Nähe Kehlmanns zur anglophonen Literatur heraus, die sie einerseits durch den Sprachwechsel und andererseits durch die Gewichtsmetaphorik unterstreicht, welche zudem wie die Auslandskritik das Bild einer schwer lesbaren deutschen Literatur zeichnet. Die Rezensentin etabliert ein auf Lesbarkeit und Qualität ausgelegtes Literaturprogramm als aussichtsreiche Basis für

\footnotetext{
450 Anon. 2007 d.

451 Ebd.

452 Polt-Heinzl 2006.
} 
kommerziellen Erfolg. Der Verkaufserfolg sowie die Fähigkeit der medialen Selbstdarstellung hätten Kehlmann - so Polt-Heinzl - zur 'Symbolfigur der [...] neuen Erzählergeneration' gemacht. ${ }^{453}$ Diese Position hätte er genutzt, um als 'Königsmacher' zu fungieren: 'Auch Kehlmann betätigt sich gern und freizügig als Referenzfigur, seine Spiegel-Eloge von Die Arbeit der Nacht etwa hat seinem Generationskollegen Thomas Glavinic die Bahn geebnet.' ${ }^{454}$ Demnach übernimmt Kehlmann, nachdem er sich selbst im Literaturbetrieb etablieren konnte, in Bezug auf Glavinic dieselbe Funktion, in der Krausser auf ihn gewirkt hatte, und setzt ebenso auf eine mediale Kollaboration zur Förderung eines poetologisch gleichgesinnten Autors.

Die literarische und mediale Zusammenarbeit Kehlmanns mit seinen Kollegen, insbesondere mit Glavinic und Krausser, wurde in Feuilletons kritisch hinterfragt. Eine Analyse der Diskussion um das Dreigestirn kann einen exemplarischen Einblick in die Lesbarkeitsdebatte der 2000er Jahre liefern. In der Furche schreibt Anton Thuswaldner, Kehlmann sei 'die Symbolfigur einer neuen Literatur der Unbekümmertheit'. ${ }^{455}$ Mit Unbekümmertheit meint Thuswaldner, dass besagte Literatur keine ideologischen Standpunkte vertritt, sondern nur mehr versucht, dem Leser die Lektüre so angenehm wie möglich zu machen. Thuswaldner bezieht einen ähnlichen Standpunkt wie Adorno und Horkheimer und kritisiert Werke, die auf Unterhaltung zielen und die Leser von zeitgenössischen Problemstellungen ablenken, wodurch er die im Literaturstreit forcierte Ablehnung einer 'Gesinnungsästhetik' unterläuft. Kehlmann bezeichnet er in diesem Zusammenhang ironisch abwertend als den 'strahlenden jungen [Gott] der österreichischen Literatur', auf den sich stellvertretend für andere Kollegen seine Kritik entlädt: 'Von anderen wäre noch zu sprechen, von Thomas Glavinic'. ${ }^{456}$ Während Thuswaldner ausschließlich auf eine poetologische Verwandtschaft der Werke Kehlmanns und Glavinics eingeht, attackiert Moritz in 'Der Avantgardist in der Medienfalle' das Gebaren Kehlmanns, durch seinen Status als Publikums-, Medien- und Kritikerliebling die Arbeiten seiner Freunde zu fördern: 'Bereits in den letzten Jahren tat sich Kehlmann keinen Gefallen damit, die Romane seiner Freunde Thomas Glavinic und Helmut Krausser publizistisch wirksam über den grünen Klee zu loben und so einen

\footnotetext{
453 Ebd.

454 Ebd.

455 Thuswaldner 2009, S. 6.

456 Ebd., S. 4 bzw. 6.
} 
zwiespältigen Eindruck zu erwecken. ${ }^{457}$ Moritz diskreditiert Kehlmanns Engagement als Marketingstrategie für die weniger prominenten Glavinic und Krausser. Dies ist sicherlich ein valider Aspekt, da alle drei Autoren dem Verkaufserfolg gewogen sind. Moritz lässt aber ästhetische Kriterien vollkommen außen vor. Krausser ging es bei seinen Äußerungen zu Kehlmanns Werk vornehmlich um die Promotion einer auf Lesbarkeit und Unterhaltung ausgerichteten Literatur, mit der Kehlmann dann der Durchbruch bei nationaler und internationaler Kritik und Leserschaft gelang. Kehlmanns Aktivitäten für die Werke seiner Kollegen sollten demnach als nächster Schritt der Etablierung von Lesbarkeit verstanden werden, die nun nicht vom Rand, sondern vom Zentrum des Literaturbetriebs aus angegangen werden kann.

Zunächst betrifft die Zusammenarbeit die Textgenese. Krausser stellt die poetologische Strategie autobiographisch in 'Ich und Kehlmann' dar: 'Kehlmann wurde nicht nur mein bewunderter Kollege, sondern auch ein enger Freund, ohne dessen Urteil ich keines meiner eigenen Bücher in Druck gehen lassen würde. ${ }^{358}$ Umgekehrt sendet Kehlmann seine Entwürfe, wie etwa sein Manuskript zur Eröffnungsrede der Salzburger Festspiele (2009) an Krausser. ${ }^{459}$ Kehlmann rekurriert in seinem Beitrag zum Text + Kritik-Band zum Werk Kraussers (2010) ebenfalls auf eine autobiographische Komponente, die er an die künstlerische Kollaboration und gegenseitige Inspiration knüpft: Er habe erleben dürfen, 'wie aus gegenseitiger Wertschätzung eine Freundschaft entstand, die zu den befruchtendsten und erfreulichsten Ereignissen seines Lebens gehört. ${ }^{460}$ Die Zusammenarbeit entsprang demnach einer gegenseitigen Achtung, die durch die poetologische Kompatibilität der beiden Werke hervorgerufen wurde. Auf dieser Basis aufbauend arbeiteten die beiden bei der Entstehung neuer Werke und bei deren Etablierung im Literaturbetrieb zusammen, was an weitere programmstrategische Zusammenarbeiten zwischen Freunden wie z.B. die Kollaboration zwischen Goethe und Schiller erinnert.

Dass auch Kehlmann versucht, möglichst viele Positionen der Literaturproduktion und -rezeption anzusprechen, zeigt die Publikationsgeschichte seines Text + Kritik-

\footnotetext{
457 Moritz 2009; beispielsweise wurde Kehlmanns Lob auf den Umschlägen von Kraussers Romanen Einsamkeit und Sex und Mitleid (2009) und Eros (2006), dem Cover der Kartongeschichte (2007) und dem Einband der Deutschlandreisen gedruckt.

458 Krausser 2008, S. 54f.

459 Vgl. Krausser 2014, S. 94.

460 Kehlmann 2010 a, S. 46.
} 
Beitrags, der in drei Fassungen an drei Publikationsorten erschien: Zuerst in der Frankfurter Allgemeinen Sonntagszeitung (30. Januar 2005), dann in Kehlmanns Essayband Wo ist Carlos Montúfar? (2005) und zuletzt 2010 in Text+Kritik. Kehlmann positioniert den Artikel in der Publizistik, im eigenen poetologischen Werk sowie in einer akademischen Zeitschrift. Durch die verschiedenen Publikationskontexte erzielt er eine umfassende Breitenwirkung für die Förderung von Lesbarkeit.

Auf Kraussers frühe Werke zurückblickend macht Kehlmann einen Wandel der Kritik geltend, den er an Kriterien des Lesbarkeitsbegriffs festmacht:

Ein Jahr später ist 'Melodien' erschienen und ist einerseits sehr erfolgreich, andererseits weit weniger, als es diesem Roman gebührt hätte: Noch reagiert der deutsche Journalismus auf prononciert narrative Texte, die große Geschichten und weite Erzählbögen entwerfen, erschrocken und mit Verdruß - und es ist nicht zuletzt Kraussers Verdienst, daß sich das inzwischen geändert hat. ${ }^{461}$

Kehlmann erkennt zu Beginn der 1990er eine Erzählfeindlichkeit im deutschen Feuilleton. Eine im aristotelischen Sinne einheitliche Handlungsführung fungiert als zentraler Punkt von Kehlmanns Ausführungen. Kraussers literarisches und poetologisches Werk sowie dessen Aktivismus sieht er als entscheidenden Beitrag zu der auch von Wittstock und der internationalen Kritik ausgemachten Wende. Somit revanchiert sich Kehlmann ebenfalls in Text+Kritik dadurch, dass er seinerseits Krausser die Vorreiterrolle zum Durchbruch ihres ästhetischen Programms zuschreibt. Er erklärt Krausser zum möglicherweise epochenprägenden Autor im wiedervereinigten Deutschland: 'In der ferneren Rückschau aber könnte es sich erweisen, daß es [...] die Zeit Helmut Kraussers [gewesen sein wird]. ${ }^{462}$ Kehlmann nutzt die prestigeträchtige Kategorie des Nachruhms, wie ihn Kant als oberstes Ziel des Schaffens ausgibt, um Kraussers Ausnahmestellung herauszuarbeiten und bekräftigt zudem die Qualität von Lesbarkeit im akademischen Kontext.

Rainer Moritz und Polt-Heinzl kritisierten neben Kehlmanns Eintreten für Krausser vor allem die Förderung von Glavinic. In der Spiegel-Kritik von Glavinics Die Arbeit der Nacht (2006) stellt Kehlmann den Roman als Höhepunkt einer Werkentwicklung dar:

\footnotetext{
461 Ebd., S. 45.
}

462 Ebd., S. 48. 
Glavinic erreiche 'die Höhe seiner Kunst' ${ }^{463}$ Kehlmann versieht Glavinics Roman mit dem Kunstattribut, wodurch er ihm zusammen mit der Höhenmetaphorik Qualität zuerkennt. Die Qualität des Romans stellt für ihn die ansprechende Umsetzung einer Mischform dar, die sich aus der E- und U-Literatur speist: Glavinic gelinge eine 'Synthese zwischen Narration und Experiment [...], psychologisch ausgefeilte, spannende Geschichten auf formal radikal innovative Weise zu erzählen.' ${ }^{464}$ Die EKategorie repräsentiert ein avantgardistisches Originalitätsstreben auf formaler Ebene, das in der Tradition und bei Krausser aufgrund der Nichtberücksichtigung der Bedürfnisse der Leserschaft für eine hohe Komplexität spricht. Die U-Kategorie vertritt das Erzählen, das den Leser durch eine exakte und realistisch plausible Gestaltung des Geschehens unterhalten soll.

Die synthetische Form sieht Kehlmann als nachhaltige Erfolgsgarantin:

Und nun also dieses Buch, zugleich aufregender Schauerroman und komplexes
literarisches Werk [...]. Ein Ereignis nicht nur in dieser Saison, sondern, man kann
es getrost voraussagen, lange darüber hinaus.
Denn 'Die Arbeit der Nacht' ist bei aller geschickt inszenierten Spannung letztlich
ein philosophischer Roman [...]. ${ }^{465}$ Der Schauerroman und Komplexität stehen oppositionell als Elemente der U- und ELiteratur. Dies bestätigt sich in Kehlmanns Beispielen: er rückt Glavinic bei der UKategorie in die Nähe von Stephen King, bei der E-Kategorie in die Nähe von Samuel Beckett, wobei Glavinics Leistung als Autor auf einer harmonischen Kombination beruht. ${ }^{466}$ Wie schon in Kehlmanns Krausser-Essay ist es auch Desiderat, lange nachzuwirken. Die Autoren streben demnach nicht nur nach der unmittelbar stärksten Wirkung auf die eigene Zeit, sondern ebenso nach dauerhaftem Ruhm, der in der Kantrezeption ein Merkmal der E-Literatur ist. Dieser soll aber im Gegensatz zu Kant dezidiert mit einem Literaturprogramm erreicht werden, das sich der Unterhaltung des Lesers verschreibt. Kehlmann zufolge wird in Die Arbeit der Nacht Unterhaltung durch eine spannende Handlung garantiert und Qualität durch die literarische Aufbereitung philosophischer Fragestellungen. Diese Strategie korrespondiert mit der postmodernen Doppelkodierung, da sie sowohl mit einer unterhaltsamen Handlung als auch mit

\footnotetext{
463 Kehlmann 2006, S. 128.

464 Ebd., S. 129.

465 Ebd.

466 Ebd.
} 
bildungsbürgerlichem Gedankengut operiert, um Qualität zu sichern und ein möglichst breites Publikum anzusprechen.

Genese und Rezeption der Arbeit der Nacht sind eines der Hauptthemen in Glavinics Literaturbetriebssatire Das bin doch ich (2007). Glavinic erzählt von einem Autor namens 'Thomas Glavinic', der unmittelbar vor der Fertigstellung von 'Die Arbeit der Nacht' steht und sich erhofft, für den Deutschen Buchpreis nominiert zu werden. Dabei reibt er sich am Erfolg seines Kollegen und Freundes 'Daniel Kehlmann' bei Publikum und Kritik. Der Titel Das bin doch ich verweist auf Kehlmann, da die Figur 'Glavinic' so auf die Nachricht reagiert, dass 'Kehlmann' in der Süddeutschen Zeitung zum 'besten Autor seiner Generation' erklärt wurde, womit 'Glavinic' seinen Qualitätsanspruch bekräftigt. 467 Ansonsten ist die Beziehung der Figuren von 'Glavinics' Neid auf und Bewunderung für 'Kehlmanns' Verkaufszahlen geprägt. Die steigenden Verkäufe der 'Vermessung' fungieren als konstantes Element der Handlungsführung und werden mit der Angst vor einem Misserfolg der "Arbeit der Nacht' kontrastiert. In dieser Hinsicht ist es konsequent, dass 'Glavinic' die Frage danach, ob die 'Vermessung' von ihm geschrieben worden sei, mit 'Nein, leider nicht' beantwortet. ${ }^{468}$ Dies beinhaltet sowohl eine ästhetische als auch eine finanzielle Dimension, was besonders während 'Glavinics' Telefongespräch mit der Mutter heraussticht, die sich darüber echauffiert, dass 'Kehlmann' den Adenauer Preis verliehen bekommt:

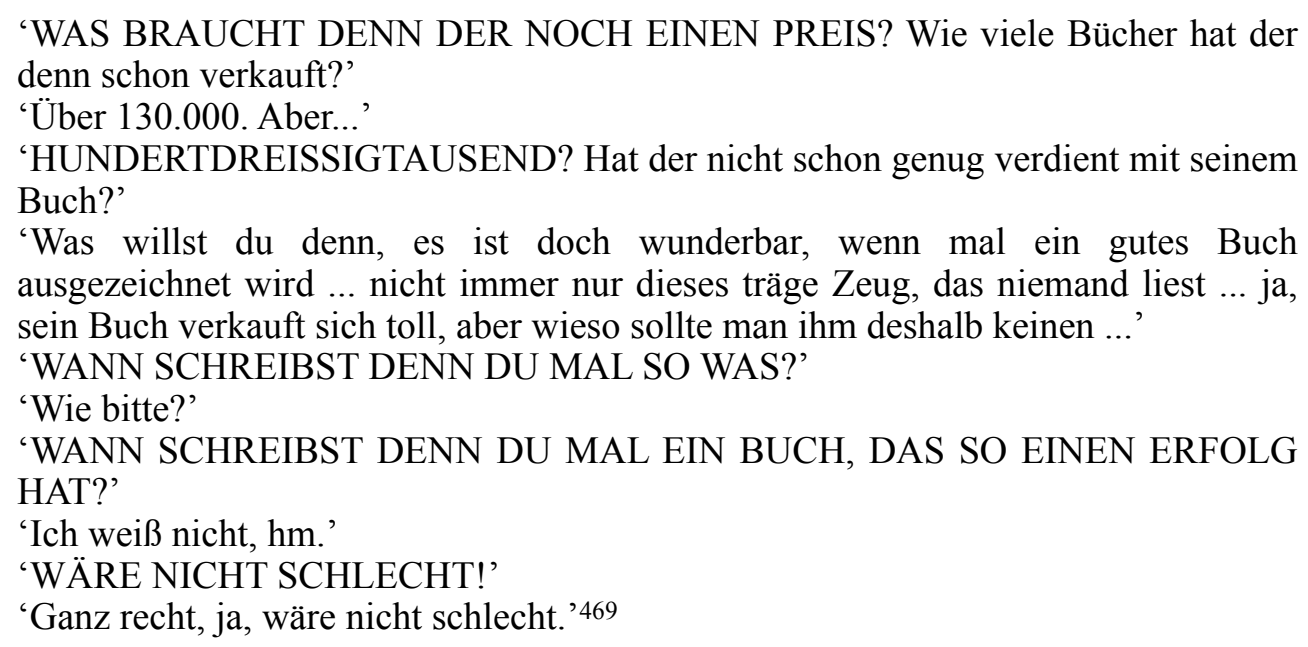

\footnotetext{
467 Vgl. Glavinic 2010, S. 41; gemeint ist Ijoma Mangolds Kritik, in der er schreibt: 'Daniel Kehlmann, die größte Begabung der jüngeren deutschen Literatur' (Mangold 2005).

468 Glavinic 2010, S. 96.

469 Vgl. ebd., S. 113.
} 
Einerseits zeigt die Stelle, dass Breitenwirkung für 'Glavinic' kommerziellen Erfolg miteinschließt. Andererseits begrüßt es 'Glavinic' wie Krausser, dass sich ein 'gutes' Buch gut verkauft hat. 'Glavinic' bezieht das Attribut 'gut' hauptsächlich auf die Vergabe von Literaturpreisen. Die auszeichnungswürdigen Kriterien gleichen dabei Kraussers Erfolgskriterien: eine unterhaltsame Literatur, die Breitenwirkung durch tatsächliche Leser- und hohe Verkaufszahlen erreicht.

Im Roman werden für Kehlmann-Glavinic Aspekte der Krausser-KehlmannZusammenarbeit verhandelt. 'Glavinic' berichtet von einem produktiven Austausch bei der Textgenese, was auf eine poetologische Affinität hindeutet. ${ }^{470}$ Zudem kritisiert 'Glavinic' am Beispiel Denis Scheck, dass die Kritik Kehlmann bei steigenden Verkaufszahlen die Qualität abspricht. ${ }^{471}$ Dadurch unterläuft er die Position Adornos und Horkheimers und tritt für eine vielverkaufte Literatur ein. Außerdem betont 'Glavinic' 'Kehlmanns' Intelligenz und Bildung, die vor allem während dessen Diskussion mit dem 'größten Starautor der westlichen Welt' - gemeint ist Jonathan Franzen - zur Geltung kommen. ${ }^{472}$

Spätestens seit der Veröffentlichung von Das bin doch ich rezipiert die Kritik Glavinic und Kehlmann zusehends als Duo. In einem Interview Glavinics mit dem Süddeutsche Zeitung Magazin heißt es:

SZ: Wie geht es Daniel Kehlmann?

G.: Kehlmann ist komischerweise jener Mensch, auf den ich am häufigsten angesprochen werde.

SZ: Weil sie befreundet sind?

G.: Weil in einem meiner Romane der Ich-Erzähler heißt wie ich und eine Romanfigur wie er. ${ }^{473}$

Diese Tendenz wurde auch auf das literarische Werk angewandt. Zur Veröffentlichung der Romane F und Das größere Wunder im Sommer 2013 bespricht Polt-Heinzl, die Kehlmann und Glavinic zuvor im Kontext einer neuen Erzählergeneration angesiedelt

\footnotetext{
470 Vgl. ebd., S. 121; dass Glavinic die Vermessung vor deren Veröffentlichung las und ebenso wie Krausser einen Verkaufserfolg prophezeite, geht aus Glavinics Artikel zu Kehlmann für den Wiener hervor, vgl. Glavinic 2013 d. 471 Vgl. Glavinic 2010, S. 206.

472 Vgl. ebd., S. 28-34; Kehlmann hat sich im Tagesspiegel lobend zur Darstellung der nach ihm benannten Figur geäußert. Die Interviewer fragen ihn, ob er es Glavinic verübelt habe, dass er als Streber rüberkomme: 'Im Gegenteil, ich sage da dauernd zynische und geistreiche Sätze und bin viel witziger als in Wirklichkeit. [...] Ein wunderbarer Roman, ich bin stolz, darin eine Figur zu sein.' (Mayer/Austilat 2008).

473 Schneider 2010, S. 24.
} 
hatte, die Romane als 'Zwillingsgeschichten'. ${ }^{474}$ Neben thematischen Übereinstimmungen macht sie die Korrespondenzen an dem metaphorisch an die Terminologie Bechers erinnernden Adjektiv 'einlässig' fest. ${ }^{475}$ Einlässigkeit stellt sie deswegen heraus, weil diese trotz einer komplexen Struktur mit wechselnden Erzählsträngen und zahlreichen intertextuellen Bezügen erreicht wird. ${ }^{476}$ Dies entspricht Polityckis Prinzip einer Simplifizierung bei gleichzeitiger Verkomplizierung, das sich aus Elementen der E- (strukturelle Komplexität und intertextuelle Verweise) und UKategorie (Einlässigkeit) speist. Entsprechend der postmodernen Theorie Regns dient das Spiel mit Referenzen dem Gewinn einer intellektuellen Leserschaft, Einlässigkeit dem Gewinn eines breiten Publikums.

Nachdem sich Glavinic im Literaturbetrieb etabliert hatte, wurde er gebeten, sich publizistisch zu Kehlmann zu äußern. Für die Lesbarkeitsdebatte ist vor allem Glavinics Beitrag zum Erscheinen der Film-Adaption der Vermessung im Magazin Wiener von Belang, in dem er vom Beginn der Freundschaft und Zusammenarbeit berichtet, den er auf die gemeinsame Vorliebe für das Erzählen zurückführt. Deswegen hätte sie 1998 bei einer Lesung ein österreichischer Germanist kritisiert, was Kehlmann auf Vorurteile im Literaturbetrieb zurückgeführt habe, die sich 'einer elitären Experimentalhermetik verschrieben hätten und alles, was so etwas wie Geschichten zu erzählen bereit war, literarisch zu verdammen pflegten.' 477 Die Bevorzugung komplexer avantgardistischer Literatur in der deutschsprachigen Literaturlandschaft schließt ein breites Publikum dezidiert aus und wertet die Erzählliteratur qualitativ ab.

Später erkennt der Germanist Helmut Gollner, dass sich das Erzählen als anzustrebendes Ideal etablierte, nimmt in seinem Interviewband zur Renaissance des Erzählens einen Praxisimpuls auf und erreicht durch die Kombination mit der Theorie eine hohe Aussagekraft in Bezug auf den Durchbruch des poetologischen Programms. Er formuliert zwei Hypothesen: erstens, dass 'Umfang und Wertschätzung der erzählenden Literatur [...] im letzten Jahrzehnt zugenommen [haben]'; zweitens, dass es unter den jungen Autoren eine Tendenz gibt, sich einer 'Indienstnahme für gesellschaftliche Aufgaben und autobiographische Absichten' zu verweigern und ein

\footnotetext{
474 Polt-Heinzl 2013, S. 6; eine Doppelbetrachtung der Entwicklung der beiden Autoren und Freunde findet sich in Philip Oltermanns Rezension von Thomas Glavinics Die Arbeit der Nacht in der London Review of Books. Dort bezeichnet Oltermann sie als 'very similar writers, a literary double act', vgl. Oltermann 2008, S. 36.

475 Polt-Heinzl 2013, S. 6.

476 Vgl. ebd.

477 Glavinic 2013 d.
} 
'Stück Welt [...] zu Literatur [zu] machen.' ${ }^{478}$ Gollner beobachtet einen Umschwung zu Gunsten des Erzählens: Er erkennt die Ablehnung einer didaktischen Funktion und macht eine realistische Gestaltungsweise geltend. Kehlmann und Glavinic zählt Gollner zu den 'künstlerisch erfolgreichsten Vertreter[n]' der jungen Erzählergeneration, wodurch er ihnen Qualität zugesteht. ${ }^{479}$ Der vom Programm der 'Neuen Deutschen Lesbarkeit' eingeleitete Paradigmenwechsel findet demnach auch in der Germanistik positive Resonanz, was den tiefgreifenden Charakter der Einflussnahme unterstreicht.

\section{Helmut Krausser}

Die Konstellation zwischen Kehlmann und Krausser spiegelt die zwischen Wittstock und Politycki. Während Kehlmann überwiegend theoretisch argumentiert, äußert sich Krausser verstärkt auch provokativ, um den Wettkampfcharakter der Etablierung seines Literaturprogramms zu unterstreichen. Diesen Wettstreit lässt er dann in seinem Roman Nicht ganz schlechte Menschen auch ins fiktionale Werk einfließen.

Krausser rekurriert im Tagebuch des August 1995 auf Termini der klassischen Rhetorik, um ein leichtes Textverständnis als Mittel zum Erreichen von internationaler Breitenwirkung zu etablieren. Obwohl er den Roman schätzt, kritisiert er an Arno Schmidts Brands Haide (1951) einen Bruch mit grammatikalischer Korrektheit und eine überambitionierte sprachliche Gestaltung: 'Eine Strafe dafür könnte die absolute Unübersetzbarkeit sein.' ${ }^{480}$ Sprachrichtigkeit stellt für Krausser eine Grundbedingung für Lesbarkeit dar, deren Nichtbeachtung eine hohe Komplexität nach sich zieht, die das Textverständnis gefährdet. Durch die Betonung des Übersetzens unterstreicht Krausser die Bedeutung von Lesbarkeit für den Weiterverkauf an ausländische Verlage, um die internationale Breitenwirkung zu fördern.

Dem kommerziellen Aspekt der Breitenwirkung steht Krausser gespalten gegenüber. Hinsichtlich seines hohen Publikationspensums gibt Krausser 1997 im Interview mit der Zeit an: 'Ich muß alle zwei Jahre ein Buch fertig haben, um mich und meine Frau zu ernähren. ${ }^{481}$ Die Sicherung des Lebensunterhalts ist demnach

\footnotetext{
478 Gollner 2005, S. 7.

479 Ebd., S. 9.

480 Krausser 1997, S. 177.

481 Burger 1997.
} 
selbstverständlicher Bestandteil des schriftstellerischen Wirkens. ${ }^{482}$ Dies stellt Krausser als Hauptgrund dafür dar, hauptsächlich Romane zu verfassen und nur sekundär in anderen Gattungen tätig zu sein. In den Deutschlandreisen führt er an, dass er sich mit zunehmendem finanziellen Verantwortungsbewusstsein dazu entschieden habe, 'Prosa (also Geld) [zu] machen'. ${ }^{483}$

Andererseits lehnt Krausser ausschließlich auf finanziellen Erfolg zielende Werke ab und wettert im Tagebuch des September gegen 'seelenlose Kommerzscheiße', der es an Niveau mangele. ${ }^{484}$ Im Interview zur Veröffentlichung des Bagarozy wehrt er sich gegen Vorwürfe, er schreibe ausschließlich des Geldes wegen: 'Und wer mich einen Kommerzschriftsteller nennt, muss einen an der Waffel haben.' ${ }^{485}$ Aus dieser entschiedenen Zurwehrsetzung spricht die Furcht vor einer Abwertung des eigenen Werkes als Teil der 'Kulturindustrie'. So eröffnet Krausser in den Deutschlandreisen eine Opposition zwischen kommerziellem Erfolg und künstlerischer Qualität und äußert, dass er es nicht verstehen könne, wenn Künstler 'Geldarbeiten' abliefern und sich damit ihren Ruf ruinieren. ${ }^{486}$ Die künstlerische Reputation fungiert gegenüber dem Verkaufserfolg als prestigeträchtigere Kategorie. Dass sich diese Pole für Krausser nicht ausschließen, zeigt seine Aussage zur Vermessung, dass sich endlich ein gutes Buch gut verkauft habe. Genügt ein Werk qualitativen Ansprüchen, verdient es sich den Markterfolg. Er teilt mit dem Programm der 'Neuen Deutschen Lesbarkeit' das Verlangen nach hohen Verkaufszahlen und die Ablehnung des idealistischen Gedankens, dass kommerziell erfolgreiche Literatur keine Kunst sein könne, fordert aber von Autoren einen hohen Qualitätsstandard ein.

Dass die Privilegierung von Qualität gegenüber Markterfolg auch Kraussers eigenem Schaffen zugrunde liegt, zeigen seine Münchner Poetikvorlesungen:

Ich möchte ein Autor für wenige sein, diesen wenigen aber will ich in besonderer Weise verbunden sein. Gerade bei meinen mir wichtigsten Büchern hab ich Entscheidungen getroffen, die jeder kommerziellen Verwertung Hohn sprechen. Gut möglich, daß ich mich damit ohne zwingenden Grund ins eigene Fleisch geschnitten habe. Aber ich fühle mich gut dabei, und das zählt enorm. ${ }^{487}$

\footnotetext{
482 Drastischer drückt er dies in der Frühlingsreise 2012 aus: 'Ich veröffentliche auch deshalb so viel, um nicht verhungern zu müssen.', Krausser 2014, S. 283.

483 Ebd., S. 16.

${ }^{484}$ Krausser 1997, S. 317.

485 Link 2014.

486 Vgl. Krausser 2014, S. 38.

487 Ebd, S. $235 f$.
} 
Krausser unterscheidet zwischen Verkaufserfolg und dem Wirken auf die Leserschaft. Letzteres wird zum Desiderat, wobei er eine umfassende Breitenwirkung zugunsten einer intensiveren Beziehung zu einer kleinen Leserschaft ablehnt. Dies ist die Variation einer Passage aus dem November 1998: 'Ich würde gerne ein Autor für wenige bleiben, dies aber dauerhaft. ${ }^{488}$ Krausser führt den Nachruhm als anzustrebende Kategorie an, der in der kantischen Tradition der E-Literatur zugeordnet werden kann. Krausser verzichtet auf die unmittelbar höchste Wirkung in der Gegenwart, erhofft sich dies jedoch durch die Nachhaltigkeit des Wirkens auszugleichen.

Innerhalb seiner gewünschten kleinen Leserschaft, zielt Krausser auf ein möglichst diverses Publikum. Im Januar benennt er die 'Verfügbarkeit aller geistigen Genüsse, auch für Schichten, die vordem niemals Zutritt erlangt hätten' als einen der großen ästhetischen Verdienste des 20. Jahrhunderts. ${ }^{489}$ Bechers Zugänglichkeitsmetaphorik steht auch bei Krausser für eine Abminderung der Schwierigkeit, die literaturfremden Lesern Vergnügen an den Werken ermöglicht. Hinsichtlich der Besetzung seiner Leserschaft merkt Krausser in den Deutschlandreisen an, dass es vollkommen egal sei, ob man vom 'Mob' geliebt werde oder von der 'Elite'. ${ }^{490}$ Krausser setzt zwei Extrempunkte der Leserschaft, die sowie alle dazwischenliegenden Gruppierungen angesprochen werden sollen.

Die Leser sieht Krausser in den Deutschlandreisen als die elementaren Akteure des Literaturbetriebs:

Ohne Publikum geht nichts. Und alles, was entsteht, ist Teil einer komplexen Unterhaltungsindustrie. Nur der allerdümmste Künstler glaubt, er wäre niemandem verpflichtet [...]. [...] Der Künstler kann, so er wichtig sein will, [den Sehnsüchten der Leserschaft] entgegenarbeiten oder strikt für sich tätig sein. Dann muß er warten und darauf hoffen, daß die Restbewohner des Planeten ihm irgendwann entgegenkommen. ${ }^{491}$

Krausser rekurriert auf die Terminologie Horkheimers und Adornos, stellt Unterhaltung und merkantiles Interesse aber nicht einer anspruchsvollen Kunst gegenüber. Letztere klassifiziert er als Teil einer 'Unterhaltungsindustrie', wodurch er die Position des

\footnotetext{
488 Krausser 2000, S. 279.

489 Krausser 2003, S. 108.

490 Krausser 2014, S. 172.

491 Ebd., S. 267.
} 
Lesers, der zum unumstrittenen Zielpunkt der Literaturproduktion wird, aufwertet. Kraussers Auffassung von Lesbarkeit speist sich demnach aus der Konstellation zwischen Autor, Werk und Leser: zugunsten der Bedürfnisse der Leserschaft verabschiedet er die idealistische Freiheit der Kunst, sich auf rein ästhetische Qualitäten zu konzentrieren. Das Schaffen soll an die Ansprüche des Publikums angepasst werden, das Krausser zum endgültigen Richter über die Bedeutung von Autor und Werk erhebt. Für Autoren eröffnet er die Möglichkeiten, sich entweder an den Bedürfnissen der Leser zu orientieren oder diese strikt auszublenden, wodurch sie sich jedoch nicht dem Verdikt der Leserschaft entziehen können.

Während Krausser, wie oben analysiert, Unterhaltung als inhärenten Bestandteil der E-Literatur etabliert, die speziell der Gewinnung der Leserschaft dient, fungiert Unterhaltung in seinen Münchner Poetikvorlesungen zudem als literarisches Politikum. Er attackiert Kollegen, die sich Unterhaltung nicht als primäre Wirkungsabsicht wählen: 'Aber wem es nicht in erster Linie darum geht, sein Publikum zu unterhalten, [...] wer in Kauf nimmt, sein Publikum zu langweilen, der hat nichts verloren in meinem literarischen Verständnis.' ${ }^{492}$ Die Indifferenz gegenüber dem Unterhaltungsbedürfnis der Leserschaft sieht Krausser als größte Schwäche der deutschen Nachkriegsliteratur an, die er darin begründet sieht, dass die Literatur ihr 'Existenzrecht nurmehr im Experiment' sah. ${ }^{493}$ Krausser wendet sich gegen ein unbedingtes Originalitätsstreben, das Unterhaltung gegen die vermeintliche Qualität experimenteller Formen tauscht.

Zudem drückt sich für Krausser ein 'Sonderweg der deutschen Literatur nach 1945' an einem Unterhaltungsdefizit aus:

[Der] Autor [...] nahm dem Leser sogar den Wunsch nach Unterhaltung übel, wollte ihn stattdessen erziehen. Das aber - ist gutgemeinte Verachtung. Die Abkehr der Leserschaft von der zeitgenössischen deutschen Literatur wurde von etlichen denn auch als kultureller Niedergang begriffen, in Wahrheit war es eine natürliche, vernünftige und nachvollziehbare Reaktion - und sehr begrüßenswert. ${ }^{494}$ 
Krausser kritisiert eine 'Gesinnungsästhetik', der eine unterhaltende Funktion in Deutschland zum Opfer gefallen ist. ${ }^{495}$ Der Leser sollte nicht mehr zur Lektüre verführt, sondern durch den erzieherischen Aspekt des docere einer Gesinnung nahe gebracht werden. Dadurch verschiebt sich die Hierarchie zwischen Autor und Leser zur Seite des Autors, der als indoktrinierende Instanz eine erhabene Position einnimmt. Krausser lehnt ein derartiges Programm ab und begrüßt, dass die deutsche Literatur zunehmend das Interesse der deutschen Leserschaft verlor. Den Misserfolg sieht er als Chance, Lesbarkeit und Unterhaltung über den Rückgewinn der Leserschaft zu legitimieren. Diesen Prozess beschränkt Krausser nicht auf Deutschland, sondern macht geltend, dass der deutsche 'Sonderweg innerhalb der globalen ästhetischen Bewegung' der deutschen Literatur im Ausland leider 'den Ruf der Unlesbarkeit eingebracht' habe. ${ }^{496}$ Wie die Besprechung der Vermessung im Ausland demonstriert, bezog sich das internationale Feuilleton tatsächlich zumeist auf Kehlmanns Leichtigkeit und Unterhaltsamkeit, die sie von deren Bild der deutschen Gegenwartsliteratur absetzte.

Die Ablehnung einer 'Gesinnungsästhetik' bedeutet jedoch nicht, dass Krausser das docere als Wirkungsabsicht ausschließt. Im Februar schreibt er: 'Unsre Gesellschaft legt keinen Wert mehr darauf, von Schriftstellern belehrt zu werden. Das geht höchstens durch die Hintertür der Unterhaltung. ${ }^{497}$ Aufgrund einer vermeintlichen Unwilligkeit des Publikums, sich belehren zu lassen, zieht Krausser wie die Gartenlaube eine Strategie heran, die der Metapher des Zuckerns gleicht: Eine wichtige Botschaft wird durch eine angenehm zu konsumierende Verpackung attraktiv gemacht. Die Belehrung spezifiziert Krausser in Galore als die Vermittlung von Bildungsgut. ${ }^{498}$ Entsprechend seines eigenen Aktivismus fordert Krausser in den Münchner Vorlesungen außerdem: 'Aufgabe eines Autors ist, ästhetisch Position zu beziehen.' ${ }^{499}$ Die Verpflichtung der Autoren zu literaturpolitischem Engagement evoziert Kraussers Aussage, dass Poetik

\footnotetext{
495 Krausser begründet seine Abneigung gegenüber einem politisch moralischen Engagement zunächst, indem er wie Wittstock eine vermeintliche moralische Überlegenheit der Schriftsteller gegenüber anderen Berufsgruppen dekonstruiert: 'Der Dichter ist keine moralische Instanz. Warum sollte der Pen-Club etwas ethisch Wertvolleres zu sagen haben, als der Metzgerverband Südhessen?' (Krausser 1995, S. 209). Im Oktober sorgt er sich um den Nachruhm: 'Politisch ist auch das Gegenteil von zeitlos. Euphemistisch ausgedrückt: Bestenfalls saisonal verwendbar' (Krausser 2000, S. 87). Schließlich argumentiert er in den Deutschlandreisen idealistisch und entwirft eine Idealvorstellung, in der über Literatur nicht 'nach Ideologien, nach Genderquote, nach Gutgemeintheit, nach politischem Engagement', sondern allein nach 'Qualität' entschieden wird; Krausser 2014, S. 274.

496 Ebd., S. 232.

${ }^{497}$ Krausser 2003, S. 294.

498 Vgl. Großmann 2004.

499 Krausser 2014, S. 246.
} 
immer auch Politik sei, was er selbst durch seinen Kampf für Lesbarkeit und Unterhaltung erfüllt.

Neben dem delectare und dem docere ist für Kraussers Autorpoetik auch das movere zentral. Krausser zeichnet in der ersten Münchner Vorlesung eine Pathostradition, die bei den klassischen Vorbildern Aristoteles, Cicero und Quintilian beginnt und bis zur Verurteilung des Pathos als nationalsozialistisches Stilmittel durch die deutsche Nachkriegsliteratur reicht. ${ }^{500}$ Krausser definiert Pathos folgendermaßen: 'Hauptmerkmal des Pathos besteht darin, einen bestehenden Sachverhalt zu überhöhen, um das von ihm ausgehende Gefühl noch $\mathrm{zu}$ steigern, $\mathrm{zu}$ verdeutlichen oder anzuklagen. ${ }^{501}$ Krausser beabsichtigt, das Leseereignis durch die Betonung emotionaler Texthaushalte $\mathrm{zu}$ veranschaulichen und $\mathrm{zu}$ intensivieren. Pathos stellt für Krausser zudem ein Qualitätsmerkmal dar: 'Der pathetische Sachverhalt bildet einen Gegensatz zum Trivialen. Der hohe Ton, um etwas auszudrücken, was mit dem üblichen Vokabular nicht ausgedrückt werden kann. ${ }^{502}$ Die Vertikalitätsmetapher der rhetorischen Stilhöhe bringt die Qualität des Pathos zum Ausdruck. Das Triviale steht mit dem Üblichen in Bezug, was die aristotelische Kategorie des Banalen evoziert. Gegen Ende des Zitats klingt mit dem aptum eine weitere rhetorische Kategorie an, die Krausser explizit darlegt: 'Es gibt angemessenes Pathos. Der Sachlage adäquat. ${ }^{503}$ Pathos muss demnach mit dem Sujet kongruieren, was eine präzise Anwendung einfordert. Ein präzise eingesetztes Pathos fungiert bei Krausser als 'zur Darstellung der Wirklichkeit [...] [notwendige Stilform]. ${ }^{504}$ Pathos avanciert zum mimetischen Mittel, das realen Lebenssituationen angemessen literarisch eingesetzt werden soll. Durch diese Doppelstrategie soll die Anschaulichkeit gesichert und die Lektüre anregend gestaltet werden.

In seinen Vorlesungen positioniert sich Krausser dezidiert als Realist:

Ich bezeichne mich selber als Realisten, das ist keine bequeme Position. Der Realist ist gezwungen, alles, was der Fall ist, ins Werk einzuarbeiten. Und das ist doch verdammt viel.

\footnotetext{
500 Vgl. ebd., S. 55-58 und 135; auch Böll spricht in seinen Frankfurter Lesungen von einer Aburteilung des Pathos als Stilmittel des Nationalsozialismus; vgl. Böll 1977, S. 87.

${ }^{501}$ Krausser 2014, S. 59.

502 Ebd., S. 75.

503 Ebd., S. 61.

504 Ebd., S. 135f; hier klingt auch Kästner Terminus der 'Präzisionsästhetik' an.
} 
Jeder andere Kunstentwurf abseits des Realismus kann als selbstgefällige Simplifikation betrachtet werden. [...] Denn nichts ist so vielfältig und kompliziert wie die Welt, in der wir leben. ${ }^{505}$

Wie Fontane etabliert Krausser die realistische als prestigeträchtigste literarische Gestaltungsweise. Während Fontane diese Erkenntnis wie Aristoteles aus der Auffassung zieht, dass der Ursprung der Kunst in der Fähigkeit und der Freude an der Nachahmung liegt, macht Krausser die Überlegenheit des Realismus an der Komplexität einer realistischen Schreibweise für den Autor fest. ${ }^{506}$

Krausser fordert in einem Gespräch (2004) neben Komplexität auch ein leichtes Textverständnis ein: 'Ich lege bei aller Komplexität des verhandelten Gegenstands Wert auf eine gewisse Oberflächenverständlichkeit'. ${ }^{507}$ Die Oberflächenverständlichkeit fungiert in Kombination mit der Komplexität, wie Krausser im Interview mit Heiner Link erklärt, als Grundstrategie einer Literatur, die sowohl anspruchsvoll sein als auch gekauft und gelesen werden soll: 'Das hat mit Raffinesse zu tun. Man baut seinen Text in vielen Schichten auf, sodass ganz oben eine Schicht erscheint, die selbst ein Depp zu verstehen glaubt.' 508 Diese Strategie erinnert an Regns doppelkodierten Vergnügensbegriff und an Kraussers Äußerungen zu Kehlmann, in denen er von einer narrativen Oberflächenstruktur und Tiefenschichten spricht. Die Oberfläche garantiert leichte Verständlichkeit, die Qualität bezieht der Text aus den komplexen Tiefendimensionen.

Allerdings erhebt Krausser in der Winterreise 2010 auch eine leicht verständliche Sprache an sich zur Qualität: 'Ich glaube, jeder halbwegs reife Künstler strebt der Einfachheit zu. Alles, was ist, so einfach auszudrükken wie möglich, bleibt das Schwierigste. ${ }^{509}$ Krausser legitimiert wie u.a. Kästner eine möglichst einfache Gestaltungsweise durch die Betonung des benötigten schriftstellerischen Vermögens als Qualität. Kraussers Befürwortung der Einfachheit entspringt zudem seiner realistischen Veranlagung. In seinen Vorlesungen entgegnet Krausser der Kritik, er habe einer Figur des Romans Eros (2006) simple und daher triviale Phrasen in den Mund gelegt: 'Aber der, der das von sich sagt, ist ein Nichtliterat. Den anders als auch mal in Phrasen

\footnotetext{
505 Ebd., S. 258.

506 Fontanes Haltung findet sich auch bei Krausser: 'Kunst ist Leben, in Form gebracht [...].' (Ebd., S. 255).

507 Fasthuber 2014.

508 Link 2014.

${ }^{509}$ Krausser 2014, S. 188.
} 
sprechen zu lassen, wäre ganz und gar unwahrhaftig, käme gekünstelt., ${ }^{510}$ Wahrhaftigkeit deutet das Desiderat einer realistischen Stilistik an, die dem Grundsatz des aptum entspricht. Kraussers Figur spricht in einer Sprache, die ihrem impliziten realen Pendant angemessenen ist. ${ }^{511}$ Krausser schöpft auch die niederen Stilebenen aus, die er als ästhetisch wertvoll ansieht, wodurch er die traditionelle höhenmetaphorische Unterteilung unterläuft, indem er normativ die realistische Gestaltungsweise als einzig angemessene etabliert.

In Kraussers Autorpoetik zeichnet sich eine Tendenz zur Mitte ab, die er im Resümee des letzten Tagebuchbands zementiert: 'Ich empfand mich stets als verlorengegangene Mitte'. ${ }^{512}$ Kraussers Kampf für die Anerkennung der mittleren Position gründet auf seiner Einschätzung, dass sie unter dem Ruf des 'Mittelmaßes [litt], dabei ist sie der gefährlichste Standpunkt, nimmt man dort doch einen Zweifrontenkrieg äußerster Angriffsfläche in Kauf.' ${ }^{513}$ Hier spiegeln sich das Spannungsfeld zwischen der E- und U-Literatur, deren jeweilige Anhänger sich an der 'mittleren' Literatur reiben. In den Tagebüchern wählt Krausser zur Beschreibung dieser Position die Metapher des 'Mix':

Meine Poetologie ist eine der radikalsten, die hierzulande vorzufinden ist, indem sie versucht, sich aus diversen Quellen zu speisen, die einander lange spinnefeind waren.

Alles bleibt, aber von allem nur das Beste, dieses will ich miteinander vermengen zu einem neuen Mix. Man kann das von der Gegenwart her durchaus als unerträglichen Mischmasch, als Eklektizismus verurteilen, aber ich glaube, daß die Zukunft immer nur der ideale Mix aus diversen Vergangenheiten ist und uns ganz anders beurteilen wird. ${ }^{514}$

Die Mischform gilt in Deutschland seit Adorno und Horkheimer als nicht adäquate Lösung für das Spannungsverhältnis zwischen der E- und U-Kategorie. Kraussers Poetologie ist deutlich vom Einfluss der Postmoderne geprägt, die eine Abkehr von der Originalität zugunsten einer aus Elementen der E- und U-Literatur gespeisten Mischform propagierte. Indem Krausser die Ausnahmestellung seiner Poetik in den deutschen Kontext einbettet, verweist er wie Wittstock auf Deutschland als verspätete

\footnotetext{
${ }^{510}$ Edb., S. 150.

511 Dazu passt auch Kraussers Aussage aus dem Tagebuch des Januar, dass nichts schlimmer sei als eine 'Überdosis Stilmittel', vgl. Krausser 2003, S. 113.

512 Krausser 2006, S. $226 f$.

513 Ebd., S. 227.

514 Krausser 2000, S. 295.
} 
Nation in Bezug auf die Postmoderne, zu deren Umsetzung Krausser in einer Vorreiterrolle beigetragen hat. Der Nachruhm fungiert als endgültige Qualitätsautorität. Den Abschluss der Wende terminiert er im April 2004: 'Zu Beginn der Neunziger standen Erzähler noch unter dem Verdacht des Publikumsopportunismus. Heute eine kaum mehr vorstellbare Diskussion. Alles, wofür ich gekämpft habe, ergab sich von selbst, als geradezu selbstverständlich. ${ }^{515}$ Die Kampfmetaphorik verweist wie der Terminus 'Literaturstreit' auf die Intensität der literaturpolitischen Debatte um Lesbarkeit seit 1990. Krausser stellt seinen eigenen Aktivismus in den Kontext eines größeren Umschwungs, der als Paradigmenwechsel von einer politisch und moralisch engagierten Nachkriegsliteratur zur Etablierung von Lesbarkeit als Qualität verstanden werden kann.

Die in den 1990ern verhandelten ästhetischen Positionen beeinflussen maßgeblich Kraussers historischen Roman Nicht ganz schlechte Menschen (2012). Krausser exemplifiziert mit seinen Protagonisten, dem Nietzscheaner Max Loewe und dem Marxisten Karl Loewe, zwei große ideologisch philosophische Strömungen des frühen 20. Jahrhunderts. Er rekurriert damit auf ein prestigeträchtiges intellektuelles Sujet. Krausser nutzt die Zwillingskonstellation außerdem dazu, um eine Metaebene in den Roman einzuziehen, die verschiedene poetologische Ansätze diskutiert. Dabei leitet er einen Emanzipationsprozess ein, der Lesbarkeit als hochwertige Kategorie etabliert. Zu Beginn des Romans heißt es: 'Karl fand unter anderem lobende Worte über den immer noch jungen Brecht, der sich politisch ja überraschend positiv entwickelt habe und nun endlich klare, verständliche Werke zur Aufklärung der Arbeiter verfasse. ${ }^{516}$ Der Marxist Karl zieht Brecht als Beispiel einer Literatur heran, die an das Programm des ‘Sozialistischen Realismus' erinnert. Oberstes Ziel ist die didaktische Erziehung des Publikums zum Sozialismus. Um auf möglichst breite Schichten wirken zu können, muss die Lesbarkeit des Werkes sichergestellt werden. Lesbarkeit wird einerseits durch die rhetorische Sprachqualität der claritas zum Ausdruck gebracht, andererseits durch den im Programm der 'Neuen Deutschen Lesbarkeit' oft synonym verwendeten Begriff der 'Verständlichkeit'.

\footnotetext{
515 Krausser 2006, S. 226.
}

516 Krausser 2012, S. 34. 
Wegen Brecht kommt es zum Streit zwischen den Brüdern. Max äußert, dass 'Brecht [...] als Autor für ihn erledigt [sei]', da jener 'eine derart trockene, kunstlose Propagandascheiße fabriziere [...]. [...] Dieser einst von Talent gesegnete Literat hatte seinen Verstand an der Garderobe der Politik abgegeben'.517 'Trocken' steht als Antonym zu mit Lesbarkeit assoziierten Metaphern wie 'süffig' und suggeriert eine zähe Rezeption. Durch den Begriff 'kunstlose Propagandascheiße' und die Gegenüberstellung von Talent und politischem Aktivismus wird eine Spannung eröffnet, die im Begriff der 'Gesinnungsästhetik' zum Ausdruck kommt: dass ein Autor wegen seines politischen Engagements die ästhetische Qualität seines Werkes vernachlässigt. Karl hingegen heißt die politische Botschaft gut und diskutiert mit Max über die ‘schlichte Sprache' des Autors. ${ }^{518}$ Während Max der einfachen Sprache einen Mangel an 'Poesie', also an künstlerischer Qualität, attestiert, nennt sie Karl 'wohltuend unpreziös'. ${ }^{519}$ Die beiden Positionen spiegeln eine Diskrepanz bei der qualitativen Einordnung einer einfachen sprachlichen Gestaltung. Während ihr Max das Gütesiegel Kunst abspricht, stellt sie für Karl gerade ob ihres Mangels an Gekünsteltem und Nähe zu der Sprache des Publikums eine Qualität dar. ${ }^{520}$

In Anschluss setzt beim ambitionierten Jungautor Max ein Prozess ein, während dessen er sich von der Vorstellung eines eminenten, prophetengleichen Autors ab- und einem egalitäreren Verhältnis mit dem Leser zuwendet. Zunächst empfiehlt er Karl Hans Falladas Roman Kleiner Mann - was nun? (1932) als Lektüre:

Genau dieses oder ein sehr ähnliches Buch hatte [Karl] schreiben wollen, traurig, witzig, raffiniert, es war kaum ein einziges Mal didaktisch, aber wahr und poetisch, dabei immer auf dem Boden der Tatsachen, geerdet, mit einem enorm liebevollen, dennoch präzisen Blick für die üblichen menschlichen Schwächen. Ein Welterfolg, ein Meisterwerk, das er nie in die Hand genommen, weil er es aufgrund seiner hohen Verkaufszahlen als trivial vorverurteilt hatte..$^{521}$

\footnotetext{
517 Ebd., S. 346.

518 Vgl. ebd. Gegen Ende des Romans führt Krausser das Beispiel Knut Hamsun an, wobei er dessen Werk bewundert und dessen politische Haltung verachtet. Eine Figur, die die literarische Qualität von Hamsuns Werk wegen dessen Affinität zum Nationalsozialismus zu schmälern sucht, wird umgehend abgekanzelt, dass sie naiv 'an einen Zusammenhang von politischer Moral und literarischer Qualität' glaube. Demnach sind Politik und Literatur indifferent, solange die Politik nicht Einzug ins Werk erhält oder die ästhetische Qualität der Literatur beeinträchtigt, was idealistische Züge trägt; vgl. ebd., S. 543f.

519 Vgl. ebd.

520 Dabei darf die Ironie nicht übersehen werden, dass der Wunsch nach einfacher Sprache mit dem bildungssprachlichen Begriff 'unpreziös’ zum Ausdruck gebracht wird.

521 Krausser 2012, S. 109.
} 
'Traurig' und 'witzig' evozieren das movere und das delectare, die Krausser in seiner Autorpoetik als bevorzugte Wirkungsabsichten benennt. Abgelehnt wird hingegen das docere, das als negativ besetzte Kategorie in Opposition $\mathrm{zu}$ den beiden anderen Funktionen gestellt wird. Ebenso wird eine didaktische Funktion als minderbewerteter Gegenpol zu den Begriffen 'wahr' und 'poetisch' etabliert, welche etwa bei Fontane und Böll als anzustrebende Qualität des Realismus gelten. Ebenso lassen sich die Termini 'raffiniert' und 'präzise' sowie die Metaphern 'auf dem Boden der Tatsachen' und 'geerdet' dem Realismus zuordnen. Sie zeigen eine mimetische Abbildung der Wirklichkeit an, die nicht unnötig künstlerisch komplex ausgestaltet ist, aber durch die akribische Ausgestaltung von Details höchsten Ansprüchen genügt. Abschließend attackiert Max ein Stigma gegenüber Verkaufserfolg, dessen internationalen Aspekt er betont und welches schließlich durch das Umdenken des Protagonisten für obsolet erklärt wird.

Zum Gegenpol einer einfacheren, realistischen Gestaltung avanciert Alfred Döblins Roman Berlin, Alexanderplatz (1929). Max' Verdikt fällt negativ aus: 'Max hatte Berlin, Alexanderplatz gelesen, einen Roman, den er bis zur Hälfte sehr ordentlich und originell, dann überambitioniert und zerfahren bis ermüdend fand. ${ }^{522}$ Originalität fungiert als Qualitätsmerkmal, was im Sinne von Max' Nietzsche-Nachfolge konsequent ist. Allerdings zeichnet sich eine positive Bewertung von Lesbarkeit ab. Max kritisiert, dass die Lektüre für den Leser wegen eines hohen künstlerischen Anspruchs zur Anstrengung wird. Eine leichte Lektüre hat für Max qualitativ einen höheren Stellenwert als eine avantgardistische und vermeintlich künstlerisch anspruchsvolle Komplexität.

Max distanziert sich von avantgardistischen Strömungen und wendet sich zusehends einer einfacheren, realistischen Literatur zu. Eine zentrale Funktion kommt dabei dem Leser zu:

Anders als Nietzsche wollte Max Loewe seine künftigen Leser nicht zu Schülern, sondern zu Verbündeten machen, die mit ihm unmittelbar und von Anfang an auf Entdeckungsreise gehen sollten, statt von Ergebnissen erzählt zu bekommen. Max hielt sich für zu jung, um glaubhaft einen Propheten zu mimen. ${ }^{523}$ 
Max zeichnet eine Autor-Leser-Konstellation, die an Ecos Prinzip des Komplizen erinnert. Durch die Ablehnung der Bezeichnung 'Schüler' wird eine hierarchische Unterscheidung zwischen Autor und Leser aufgehoben und ein didaktischer Anspruch abgelehnt. Der 'Verbündete' steht demnach wie Ecos Komplize für eine Gleichberechtigung zwischen Autor und Leser. Max untermauert dies durch die Absage an eine, durch die religiöse Metapher des Propheten ausgedrückte, überhöhte Position des Autors. Die Entdeckungsreisen-Metapher erinnert an Gutzkows Anspruch, den Leser in unbekannte Gefilde mitzunehmen. Allerdings erhebt Gutzkow im Gegensatz zu Kraussers Figur einen Führungsanspruch, der den Autor hierarchisch eindeutig oberhalb des Lesers ansiedelt.

Dass die Entdeckungsreise an realistische Gegebenheiten gebunden ist, zeigt eine Stelle, in der Max Feedback zu seinem Romanentwurf erhält: 'Max Loewe mußte, wie jeder angehende Romancier, lernen, daß der Leser vor allem liest, was er auf sich bezieht, was ihn bestätigt oder ihm sofort einleuchtet. ${ }^{924}$ Der Erzähler formuliert die Notwendigkeit, dass fiktive Figuren den Lesern ein Identifikationspotenzial anbieten müssen, um auf ihn wirken zu können. Die Lichtmetapher signalisiert, dass dazu ein leichtes Textverständnis als Voraussetzung nötig ist. Die beiden Strategien werden als textuelle Basis für Breitenwirkung etabliert. In der gleichen Passage wirft der Rezensent Max vor, seine Figuren in trivialen Szenarien wie Verfolgungsjagden, Drogen- und erotischen Exzessen agieren zu lassen. Max entgegnet:

Wenn es derlei gab, konnte es nicht trivial gennant werden. [...] Wo käme man hin, wenn man beim Schreiben eines Romans peinlich genau vermeiden wollte, Ähnlichkeiten mit lebenden Menschen zu riskieren? Eher müsse man als Realist doch bestrebt sein, die Welt so abzubilden, wie sie war. ${ }^{525}$

Max verwendet dieselbe Rechtfertigungsstrategie, mit der Krausser den Trivialitätsvorwurf gegenüber Eros revidiert. Der realistische Ansatz wird dann zur Legitimation der Abbildung gewisser Vorkommnisse, wenn diese in der Realität fundiert sind. Ebenso kommt ihm eine Schutzfunktion gegen die Trivialität zu. Max' Entwicklungen von einer komplexen Genieästhetik hin zur Lesbarkeit wird hier durch 
das klare Bekenntnis zu und der Einforderung der konsequenten Umsetzung einer realistischen Gestaltungsweise abgeschlossen.

Dass Max’ Realismusbegriff auf Unterhaltung zielt, zeigt eine Diskussion mit dem Anarchisten Zanoussi. Jener äußert, dass Unterhaltungsromane, 'oftmals unterschätzt würden, wo sie ihre inneren Qualitäten doch vor viel mehr Lesern beweisen müßten als all das hochgestochene Zeug. Ein solches Denken faszinierte Max, forderte zugleich seinen Widerwillen heraus, als müßte er sich davon abgestoßen fühlen. ${ }^{526}$ Die Stelle beinhaltet die Auffassung, dass unterhaltende Literatur ein größeres Publikum findet als eine künstlerisch ambitionierte. Dadurch, dass Krausser Max das implizierte Vorurteil gegenüber Unterhaltung hinterfragen lässt und dieser sich auf die Seite der Unterhaltung schlägt, findet eine Reevaluierung des Werts von Unterhaltungsliteratur statt, die dem Stigma gegen die 'Kulturindustrie' entgegenwirkt. ${ }^{527}$ Breitenwirkung wird nicht wie in der Dialektik der Aufklärung als Indiz für Trivialität gewertet, sondern als Kategorie, die es einem Werk schwieriger macht, in seiner Rezeption qualitativ zu reüssieren. Die Kritik wird als Hoheitsinstanz abgelöst, eine Pluralität der Geschmäcker akzeptiert und die breite Leserschaft als Instanz zur qualitativen Bewertung von Literatur aufgewertet.

Nicht ganz schlechte Menschen fungiert wie die poetologischen Passagen der Tagebücher als Apologetik des eigenen Schaffens. Durch Max’ Entwicklung diffundiert Kraussers Kampf für die Lesbarkeit selbstreflexiv ins literarische Werk. Krausser transportiert die Lesbarkeitsdebatte in ein anderes Medium und macht sie einem womöglich größerem Publikum zugänglich, nämlich den Lesern, die sich nicht mit poetologischen Fragestellungen auseinandersetzen. Die Lesbarkeitsdebatte erhält somit einen größeren Resonanzraum und wird breitenwirksamer. Dazu entwirft Krausser ein Mini-Corpus, das weitere historische Analysen der Lesbarkeit, etwa am Beispiel von Falladas Kleiner Mann - was nun?, inspirieren könnte. Abschließend wird Lesbarkeit durch die selbstreflexive Herangehensweise nicht nur als Qualitätsmerkmal etabliert, sondern auch Selbstreflexivität als Qualitätsmerkmal von auf Lesbarkeit gründender Literatur.

\footnotetext{
526 Ebd., S. 371.

527 Ulrich Rüdenauer bestätigt in seiner Rezension die Aufwertung von Unterhaltung in der deutschen Kritik: 'Der Roman ist weder neu noch groß, dafür aber unterhaltsam.' (Rüdenauer 2012). Rüdenauer schließt Unterhaltung von einer durch die Größenmetaphorik ausgedrückten Qualitätsliteratur zwar aus, sieht sie jedoch als eigenständiges Qualitätsmerkmal, was darauf hindeutet, dass Unterhaltung bei der Kritik als Qualität gilt, die von traditionellen Wertungshierarchien unabhängig ist.
} 


\section{Daniel Kehlmann}

Aufgrund seines Erfolgs entwickelte Kehlmanns Werk die größte Wirksamkeit hinsichtlich der Etablierung von Lesbarkeit als Qualität. Im Rahmen der prestigeträchtigen Frankfurter Poetikvorlesungen rekurriert Kehlmann in Kommt, Geister (2014) auf das im Literaturstreit umkämpfte Spannungsfeld zwischen Unterhaltung und politisch-moralischem Engagement in der deutschen Nachkriegsliteratur. Stellvertretend für die Literatur diskutiert er die Unterhaltung am Beispiel der Filme Peter Alexanders; die Gruppe 47 dient ihm als Beispiel für künstlerisches Engagement, wobei er beide vor dem Hintergrund der nationalsozialistischen Vergangenheit durchleuchtet. Kehlmann lehnt sowohl ein vermeintliches 'Politikergehabe' und 'Moralisieren' als auch die Peter-Alexander-Filme, die er in Anspielung auf Adorno und Horkheimer als Teil einer deutschen 'Unterhaltungsindustrie' der Nachkriegszeit bezeichnet, ab. ${ }^{528}$ Er kritisiert, dass die Filme 'schlecht' und 'langweilig' sind, wodurch er das Fehlen eines ästhetisch anspruchsvollen Unterhaltungsfilms bzw. einer qualitativ hochwertigen Unterhaltungsliteratur in der Nachkriegszeit moniert. ${ }^{529}$ Zudem bemängelt er, dass die Filme die 'Wirklichkeit [...] radikal aus[sperren]' und formuliert so ein Realismusdesiderat. ${ }^{530}$ Kehlmann folgert am Beispiel von Ingeborg Bachmanns Frankfurter Poetikvorlesungen, dass die schlechte Unterhaltung und die politische Didaktik dazu geführt hätten, dass die deutsche Literatur den Anschluss an die Weltliteratur verloren hätte und interpretiert Bachmanns Ausführungen als Kampf um den Anschluss an die internationale Literatur. ${ }^{531}$ Dies spricht für die Ausrichtung seiner Literatur auf einen globalen Kontext.

In Reportagen im Guardian und dem Times Literary Supplement führt Kehlmann seine Gedanken zur Nachkriegsliteratur genauer aus. Im GuardianArtikel, der die Vermessung als Wendepunkt für die deutsche Gegenwartsliteratur ansieht, kritisiert Kehlmann das elitäre Gebaren der Gruppe 47, 'that only high culture counts. ${ }^{532}$ Er tritt für eine Aufwertung der Populärkultur ein, um an internationalen

\footnotetext{
528 Ebd., S. 12.

529 Ebd.

530 Ebd., S. 18.

531 Ebd., S. 10

532 Harding 2006.
} 
Tendenzen, vor allem in der anglo-amerikanischen und der südamerikanischen Literatur, anzuschließen und suggeriert zugleich eine Rückständigkeit der deutschen Literatur. ${ }^{533}$ Dezidiert fordert Kehlmann im Times Literary Supplement seine Kollegen auf: 'to get over the self-imposed heaviness of German letters, an old literary generation's guilt and obsession with the war.' ${ }^{534}$ Kehlmann setzt die literarische Auseinandersetzung der älteren Generation mit dem Zweiten Weltkrieg der Leichtigkeit entgegen. Das Streben nach Leichtigkeit benennt er als 'intention to revive German literature as popular entertainment'. ${ }^{535}$ Wie in seinen Frankfurter Vorlesungen setzt Kehlmann Unterhaltung und Didaktik in Opposition und äußert dezidiert eine Inklination für Unterhaltung, die er an den Wunsch knüpft, möglichst viele Leser anzusprechen. ${ }^{536}$

Neben einer politischen Funktion lehnt Kehlmann im Interview mit der Presse auch ein moralisches Engagement ab, indem er mahnt 'wie problematisch es ist, Künstler als moralische Autoritäten zu sehen' und dies damit begründet, dass manche der bedeutendsten Schriftsteller des 20. Jahrhunderts politisch radikale Positionen vertreten hätten. ${ }^{537}$ Dies bettet er in eine allgemeine Befragung der Position der Schriftsteller in der Gesellschaft: 'Jeder Bürger ist berechtigt, seine Meinung zu äußern, aber die eines Romanciers hat nicht per se größeres Gewicht als die eines Zahnarztes oder Ingenieurs. ${ }^{538}$ Kehlmann dekonstruiert eine etwaige Überlegenheit der Autoren gegenüber anderen Berufsgruppen und befreit sie dadurch von dem Debet, politisch und moralisch Stellung beziehen zu müssen.

Ein ambivalenteres Verhältnis pflegt Kehlmann zur Belehrung im Sinne der Verbreitung von Bildungsgut. Im Interview mit dem Falter (2009) entgegnet er auf die Annahme, dass viele die Vermessung gelesen hätten, um etwas über Humboldt und Gauß zu lernen:

Die 'Vermessung' wurde jedenfalls auf eine sehr eigenartige Weise als Gegengift zur Pisa-Katastrophe betrachtet und - auf der anderen Seite - von einer konservativen Publizistik stark angegriffen, weil sie angeblich zu respektlos mit der deutschen Tradition umgeht. Ich hatte immer das Gefühl, dass diese Leute das

\footnotetext{
533 Vgl. ebd.

534 Roth 2006.

535 Ebd.

536 Die Auflösung dieses Spannungsfeldes zugunsten der Unterhaltung postuliert Kehlmann zudem in seinen Göttinger Poetikvorlesungen (2006) und in Gollners Interviewband; vgl. Kehlmann 2011 c, S.128f und Gollner 2005, S. 36.

537 Klauhs 2009, S. 63.

538 Ebd.
} 
Buch besser verstanden haben als jene, die es als Bildungsbuch für die Schullektüre empfehlen. 539

Kehlmann befürwortet eine kritische Auseinandersetzung mit Bildungsgut. Da die Reflexion über Bildungsgüter ein gewisses Maß an Wissen voraussetzt, avanciert Bildung zu einem immanenten Bestandteil von Kehlmanns Büchern. Dies unterstreicht er im Interview mit dem Magazin $D u$ (2009), in dem es ihn stört, dass die Kritik seine Bildung kritisch hinterfrage: 'Einem Schriftsteller vorzuwerfen, dass er gebildet sei, ist aber in etwa so, als würde man einem Arzt seine Anatomiekenntnisse verübeln.' ${ }^{540}$ Bildung fungiert als grundlegende Voraussetzung von Autorschaft und als elementarer Baustein von Kehlmanns Werken. Kehlmann vertritt jedoch keinen Anspruch, Kenntnisse an die Leserschaft weiterzugeben. ${ }^{541}$

Der Verzicht auf eine didaktische Funktion bedeutet jedoch nicht, dass Kehlmann keine Wirkung auf die Leser auszuüben gedenkt. Im Gesprächsband Requiem für einen Hund (2010) benennt er einen Wunsch nach Ausstrahlung: 'Und ausstrahlen heißt Leute erreichen, gelesen, wahrgenommen werden. Und wenn einem das gelingt, dann ist es auch die Erfüllung einer Sehnsucht.. ${ }^{542}$ Kehlmann etabliert das Gelesenwerden und den Bekanntheitsgrad in der Öffentlichkeit als privilegierte Ziele. Die Leserschaft fungiert als Königsmacher, die dieses Verlangen durch ihre Lektüre- bzw. Kaufentscheidung erfüllen oder versagen kann, wodurch deren Position im Literaturbetrieb aufgewertet wird. Im Hinblick auf ein Zielpublikum äußert Kehlmann im Tagesspiegel, dass er bei der Konzeption nicht 'zielgruppenorientiert [...] denken' könnte. ${ }^{543}$ Kehlmann impliziert das Desiderat, eine breite und diverse Leserschaft anzusprechen. Dieses knüpft er im Interview mit der Presse zudem an den traditionell prestigeträchtigen Nachruhm: Er wünsche sich 'Bücher [...], die nicht schon nach zwei Jahren vergessen werden. [...] Zu dem Ausstrahlen-Wollen gehört der Wunsch, von möglichst vielen Menschen gelesen zu werden. Insofern wünscht man sich schon Dauerhaftigkeit'. ${ }^{544}$ Gelesenwerden und Ausstrahlung stellen die bevorzugten Kategorien der

\footnotetext{
539 Nüchtern 2009, S. 25.

540 David 2009, S. 29.

541 Eine Ausnahme macht er für seinen Roman Ich und Kaminski (2003), in dem er aufgrund der kritischen Auseinandersetzung mit dem Kulturjournalismus eine 'gesellschaftliche Botschaft' erkennt; vgl. Maar 2008, S. 66.

542 Kehlmann/Kleinschmidt 2010, S. 136.

543 Meller 2006.

544 Klauhs 2009, S. 62.
} 
Breitenwirkung dar, wobei Kehlmann diesen hier eine diachronische Komponente hinzufügt, um über einen längeren Zeitraum möglichst viele Leser zu erreichen.

Gegenüber dem finanziellem Aspekt der Breitenwirkung nimmt Kehlmann in der Dankesrede zur Verleihung des Welt-Literaturpreises (2007) eine differenzierte Haltung ein, die er in Anlehnung an den ungarischen Schriftsteller Imre Kertész als 'Glückskatastrophe' ausdrückt. ${ }^{545}$ Die positive Seite sieht er des Öfteren in einer 'Querfinanzierung'.546 Darunter versteht er: 'Das Wichtigste ist ein gewisses Sicherheitsgefühl. Der Eindruck, dass man für eine ganz schöne Zeit seine Arbeit machen kann, ohne dass einen irgendjemand davon abhalten kann. ${ }^{547}$ Der kommerzielle Erfolg garantiert eine finanzielle Absicherung, die eine alleinige Konzentration auf die Literatur ermöglicht und dadurch Qualität sichert. ${ }^{548}$ In der Presse sieht Kehlmann einen weiteren positiven Aspekt: 'Und der Hauptzweck des Bestsellergeldes ist ja, dass ich die Freiheit habe, künstlerisch über lange Zeit zu machen, was ich will.' ${ }^{549}$ Die finanzielle Unabhängigkeit sichert demzufolge künstlerische Autonomie, worin sich sowohl das Idealbild des freien Schriftstellers als auch die komplexen Bedingungen des Literaturmarktes, die Einfluss auf das Werk nehmen, spiegeln.

Die Abhängigkeit von Marktgegebenheiten beschäftigen Kehlmann im Hinblick auf einen Qualitätsverlust: 'Wenn Autoren vom Markt leben müssen, müssen sie eine Art von Büchern schreiben, die auf den Markt zielen und vielleicht ihr eigenes künstlerisches Kalkül verletzen. ${ }^{550}$ Kehlmann rekurriert insofern auf die Vorbehalte der 'Kulturindustrie', als dass er eine Anpassung an Marktbedingungen als möglichen Grund für einen ästhetischen Kompromiss anführt. Er disqualifiziert erfolgreiche Werke nicht automatisch, hegt aber dennoch Zweifel am eigenen Werk. In der Literatursendung Das blaue Sofa wundert sich der Moderator Wolfgang Herles darüber, dass sich Kehlmann um die Qualität des eigenen Werks sorge, wo er doch einen Weltbestseller hingelegt habe. ${ }^{551}$ Kehlmann lehnt eine solche Gleichsetzung von Verkaufserfolg und Qualität ab: 'Man weiß ja trotzdem nicht wirklich [...], wie viel das eigentlich taugt,

\footnotetext{
545 Kehlmann 2011 b, S. 174.

546 U.a. Paterno 2006, S. 135; Lovenberg 2008.

547 Meller 2006.

548 Dies sei zudem dadurch gewährleistet, dass er die Anzahl an Lesungen verringern könne, um sich auf die Schreibarbeit zu konzentrieren; vgl. Paterno 2006 und Nüchtern 2009 sowie Kehlmann 2011 c, S. 136.

549 Simon 2008, S. 35.

550 Polt-Heinzl 2013, S. 6.

551 Herles 2013 a.
} 
was man gemacht hat. ${ }^{552}$ Wie Wittstock sieht Kehlmann Markterfolg zwar als Indikator, aber nicht als Garanten für Qualität und setzt sich gegen eine solche Verknüpfung ein. ${ }^{553}$ Einerseits kritisiert er in der Presse eine Stigmatisierung von vielverkauften Romanen, indem er sich darüber freut, dass 'das Feuilleton mein Buch gegen dessen eigenen Erfolg verteidigt [hat]. ${ }^{554}$ Andererseits zeigt er sich gleichermaßen begeistert, dass die Annahme, Niveau finde kein großes Publikum, durch die Vermessung widerlegt worden sei, und sich auch seine Kollegen über den Erfolg von Qualität auf dem Absatzmarkt freuten. ${ }^{555}$ Dies trifft, wie Kraussers und Glavinics Reaktionen zeigen, zu. In der Dankesrede folgert er: 'Noch immer macht mich jede Anerkennung, die [die Vermessung] nicht als nur Verkaufsschlager, sondern als geformtes literarisches Kunstwerk erhält, stolz' ${ }^{556}$ Kehlmann ist sich der seit Adorno und Horkheimer in Deutschland verankerten Ächtung von erfolgreichen Werken bewusst. Er sieht Verkaufserfolg grundsätzlich positiv, ordnet ihn jedoch der Qualität und dem Ruf eines anspruchsvollen Künstlers unter.

Kehlmanns Auffassung einer qualitativ ansprechenden Literatur gründet auf einer realistischen Gestaltungsweise. In Kommt, Geister benennt er eine mimetische Textgestaltung als Grundvoraussetzung aller Literatur. Er referiert über die Shakespeare-Figuren Prospero und Oberon. Oberon sei wie Prospero kein Künstler, weil 'seine Werke [...] nicht mehr mimetisch [sind]; er schafft nicht eine beschränkte Nachbildung der Wirklichkeit, sondern er gestaltet diese selbst.' ${ }^{557}$ Mimesis dient Kehlmann als zentraler Kunstbegriff über den sich der Kunststatus erst definiert.

Kehlmanns Ausführungen zur Handlungsgestaltung beziehen sich deutlich auf die aristotelische Tradition. Er spricht von 'narrative[r] Stringenz', die er folgendermaßen definiert:

Handlung ist zweckbestimmte Kausalität. Ein Ereignis folgt aus einem anderen, zugleich aber wirkt auf die Ereignisse noch eine Kraft von außen: der Wille des Erzählers. [...] Handlung ist [...] die Substanz der Erzählung [...]. Das Prinzip Plot ist unverzichtbar, aber es ist nie ganz unproblematisch. Wird der ordnende Wille des Autors zu deutlich, wirkt eine Geschichte zurechtgemacht und konstruiert. Der Grund dafür ist eben der Umstand, dass es Handlung nur in Geschichten gibt und

\footnotetext{
552 Ebd.

553 Konsequenterweise argumentiert Kehlmann, dass umgekehrt auch Qualität kein Garant für Erfolg ist, sondern komplexe Marktmechanismen ineinandergreifen müssen; vgl. Maar 2008, S. 61.

554 Simon 2008, S. 33.

555 Vgl. Paterno 2006.

556 Kehlmann 2011 b, S. 177.

557 Kehlmann 2015, S. 86.
} 
nicht in der Wirklichkeit. [...] Schon Aristoteles unterscheidet die Wirkursache von der Zweckursache, [...] jene ist der Grund, aus dem, diese der Zweck, zu dem etwas geschieht. Beim Erzählen [...] bleiben Zwecke unverzichtbar, und der Erzähler spielt, ob er das will oder nicht, in seinem eigenen zweitklassigen Kosmos Gott. In der Realität walten die Gesetze der Physik, in der Erzählung aber die Zwecke der Dramaturgie. ${ }^{558}$

Kehlmann steht für eine kausal schlüssige Handlungsfolge ein, die er als wichtigste Kategorie etabliert, was Aristoteles' Einordnung als 'erste[r] und wichtigste[r] Teil der Tragödie' entspricht. ${ }^{559}$ Zudem sieht Kehlmann eine geformte Handlung als kompensatorische Ordnung für die Unordnung der Realität an, was im Sinne von Fontane für eine erzählerische Wahrhaftigkeit spricht. ${ }^{560}$ Dem Autor weist Kehlmann eine gottgleiche Position zu, die sich jedoch nicht wie bei den ebenfalls auf religiöse Terminologie zurückgreifenden Expressionisten in einer Überhöhung der Autorgegenüber der Leserposition ausdrückt. Wie in der aristotelischen Poetik und Rhetorik erscheint der Begriff des 'Zwecks' als zentraler Aspekt, den Kehlmann an den Begriff der 'Dramaturgie' knüpft. In Die Wahrheit lügen formuliert er zunächst eine dem vorhergehenden Zitat entsprechende Form der Handlungsführung. ${ }^{561}$ Dann benennt er den Zweck dieser Erzählstrategie. Es geht Kehlmann darum, 'alle Techniken einzusetzen, um am Ende im Leser [...] dieses gleiche [...] Gefühl innerer Folgerichtigkeit zu erzeugen.' ${ }^{562}$ Die stringente Handlungsführung nutzt Kehlmann als Mittel dazu, dem Leser die logischen Zusammenhänge klar zu machen und dadurch die Lektüre zu erleichtern.

Eine ähnliche Strategie verfolgt Kehlmann bei der Gestaltung von einzelnen Szenen. In seinen Göttinger Poetikvorlesungen Diese sehr ernsten Scherze (2006) erörtert er die Bedeutung einer detaillierten und fehlerfreien Szenengestaltung im Hinblick auf den Leser und macht geltend, dass nur 'wenn der Autor eine Szene sieht, dann sieht sie auch der Leser.' 563 Die Wortwahl erinnert an den rhetorischen

\footnotetext{
558 Ebd., S. 55 und 150f.

559 Aristoteles 1994, S.25.

${ }^{560}$ Dieselbe Position vertritt Kehlmann in Wo ist Carlos Montúfar?: 'Ein Erzähler operiert mit Wirklichkeiten. Aus dem Wunsch heraus, die vorhandene nach seiner Vorstellung zu korrigieren, erfindet er eine zweite, private, die in einigen Punkten und vielen gut versteckten von jener ersten abweicht.' Später heißt es: 'Erzählen, das bedeutet, einen Bogen zu spannen, wo zunächst keiner ist, den Entwicklungen Struktur und Folgerichtigkeit gerade dort zu verleihen, wo die Wirklichkeit nichts davon bietet - nicht um der Welt den Anschein von Ordnung, sondern um ihrer Abbildung jene Klarheit zu geben, die die Darstellung von Unordnung erst möglich werden läßt.' (Kehlmann 2010 c, S. 10 und S. 14). 561 'Es gibt ein Gefühl von Notwendigkeit in einer Geschichte. Das wird hervorgerufen durch die Komposition. Dieses und jenes geschieht diesen und keinen anderen Leuten in dieser Reihenfolge und auf diese Art' (Gollner 2005, S. 30).

562 Ebd., S. 30f.

563 Kehlmann 2011 c, S. 133.
} 
Hauptzweck der 'Anschaulichkeit', die die Autoren dazu anhält, ein möglichst detailliertes und wahrscheinliches Gesamtbild zu zeichnen, um dem Leser möglichst leicht Zugang zum Text zu verschaffen.

Richtigkeit und Präzision stehen bei Kehlmann auch in Bezug auf die sprachliche Gestaltung im Fokus. In den Ernsten Scherzen schreibt er: 'Ich fand Literatur immer am faszinierendsten, wenn sie nicht die Regeln der Syntax bricht, sondern die der Wirklichkeit. ${ }^{564}$ Kehlmann akzeptiert Brüche auf der inhaltlichen Ebene, um die Grenzen zwischen Realität und Fiktion zu destabilisieren, die Sprachregeln sollen jedoch eingehalten werden. ${ }^{565}$ Kehlmanns Plädoyer für Sprachrichtigkeit entspricht dem ersten rhetorischen Stilprinzip, das wie auch bei Krausser eine Grundvoraussetzung für Lesbarkeit darstellt. Rhetorische Begriffe finden sich auch in weiteren Äußerungen zur Sprache. Im Interview mit der Stuttgarter Zeitung (2007) gibt Kehlmann an, 'genau' und 'exakt' mit der Sprache zu sein. ${ }^{566}$ Die Adjektive lassen sich auf zweierlei Weise lesen: Erstens, bezüglich der wörtlichen Quantität, einen Sachverhalt mit möglichst wenigen Worten darzustellen (brevitas); Zweitens, hinsichtlich der Qualität der Darstellung, als eine möglichst eindeutige Ausdrucksweise (perspicuitas vs. ambiguitas). Beide Auslegungen korrespondieren mit Kästners 'Präzisionsästhetik', die für Lesbarkeit steht. Kehlmann schreibt in den Ernsten Scherzen ebenfalls, dass Literatur in formaler Perfektion aus 'möglichst brillanten, tänzelnd überraschenden Sätzen bestehen' müsse. ${ }^{567}$ Die Verwendung von 'tänzelnd' und 'brillant' kombiniert eine spielerische Leichtigkeit mit dem Willen zu einer ambitionierten stilistischen Gestaltung, worin sich Anklänge an die aristotelische Mischung von Klarheit und NichtBanalem finden. Die Kombination aus Sprachrichtigkeit, und dem Willen zu brillanten Sätzen, lässt sich als Form einer stilistisch hochwertigen und zugleich fließend lesbaren Sprache lesen, die dem Rezipienten keine großen Hindernisse entgegensetzt.

Kehlmanns Autorpoetik kann im Sinne des Guardian-Artikels als Eintreten gegen den Ernst der deutschen Gegenwartsliteratur gelten. ${ }^{568}$ Dabei rekurriert er auf bewährte Mittel der Realismustradition, um die laut ihm im Literaturbetrieb verbreitete

\footnotetext{
564 Ebd. S.137.

565 Es geht Kehlmann um den Einfluss der südamerikanischen Autoren und die Verwendung von fantastischen Elementen. Jene lässt auch Aristoteles in seiner Poetik zu, sodass diese mit dem Mimesis-Gebot zu vereinbaren sind; vgl. Gollner 2005, S. 31-34 bzw. Aristoteles 1994, 24, S. 83.

566 Vgl. Anon. 2007 b.

567 Vgl. Kehlmann 2011 c, S.134f.

568 Im Interview sagt er der Frankfurter Allgemeinen Zeitung, dass er in der deutschen Gegenwartsliteratur das 'Spielerische und [die] Leichtigkeit' vermisst, vgl. Lovenberg 2006.
} 
Auffassung 'Realismus=Erzählen=altmodisch' zu dekonstruieren. ${ }^{569}$ Es geht ihm um einen Paradigmenwechsel, der eine nach Lesbarkeit und Unterhaltung strebende Erzählliteratur als qualitativ hochwertig etabliert. Diesen Wechsel sieht er im Erfolg der Vermessung bestätigt. In Wo ist Carlos Montúfar? schreibt er, dass die

\begin{abstract}
deutsche Literaturkritik vorübergehend aufgehört hat, das Erzählen totzusagen [...]. Dieselben, die vor ein paar Jahren mit Adornozitaten nach jedem erzählerischen Absatz schleuderten, überschlagen sich heute vor Begeisterung, wenn sie die einfachsten realistischen Schilderungen aus dem Alltagsleben lesen. ${ }^{570}$
\end{abstract}

Kehlmann macht eine Umerziehung der Kritik geltend, die sich einer realistischen Erzählliteratur zugewandt hat. Dies macht er an einem Wandel in der Adorno-Rezeption fest. Sein Verweis auf die Temporarität stellt zudem die Bedeutung der historischen Einordnung des Lesbarkeitsparadigmas zu Beginn des 21. Jahrhunderts heraus.

Dass Kehlmann mit der Vermessung einen qualitativen Anspruch verfolgt, zeigt sich zunächst an der Wahl eines prestigeträchtigen, bildungsgütlichen und intellektuellen Sujets. ${ }^{571}$ Alexander von Humboldt und Carl Friedrich Gauß als Protagonisten einer fiktionalen Doppelbiografie zu wählen, zieht strukturelle Konsequenzen nach sich, die nicht zwingend mit der von Aristoteles eingeforderten Handlungsführung korrespondieren. Im ersten Kapitel fungiert der von Humboldt organisierte Berliner Naturforscherkongress (1828) als Zeitpunkt der Rahmenhandlung. Dann eröffnet Kehlmann in den folgenden neun Kapiteln eine umfangreiche Analepse, in der abwechselnd Humboldts und Gauß' Lebensgeschichten chronologisch vom Kindesalter bis hin $\mathrm{zu}$ deren wissenschaftlichen Errungenschaften erzählt werden. Beide Erzählstränge verlaufen gleichberechtigt nebeneinander und werden im elften Kapitel im Rahmen des Kongresses zusammengeführt. Dies spricht für eine komplexere Gestaltung der Handlungsführung als die in der aristotelischen Poetik angelegte.

Es gilt, die Ausarbeitung der Übergänge zwischen den Zeitebenen zu analysieren. Die Gegenwartsebene wird in den ersten Sätzen des Romans explizit etabliert:

\footnotetext{
569 Gollner 2005, S. 31.

570 Kehlmann 2010 c, S. 141.

571 Zur Wahl und Gestaltung der Protagonisten im Hinblick auf die Publikumswirksamkeit siehe auch Braun 2013.
} 
Im September 1828 verließ der größte Mathematiker des Landes zum ersten Mal seit Jahren seine Heimatstadt, um am Deutschen Naturforscherkongreß in Berlin teilzunehmen. Selbstverständlich wollte er nicht dorthin. Monatelang hatte er sich geweigert, aber Alexander von Humboldt war hartnäckig geblieben, bis er in einem schwachen Moment und in der Hoffnung, der Tag käme nie, zugesagt hatte. Nun also versteckte sich Professor Gauß im Bett. ${ }^{572}$

Die Jahreszahl begründet das historische Setting. Zudem wird mit Berlin die Lokalität eingeführt. Kehlmann benennt und charakterisiert ansatzweise die beiden Hauptfiguren, wobei besonders bei Gauß der narratologische Ansatz des Romans, die Figuren als wissenschaftliche Genies und als sozial inkompetente Privatpersonen darzustellen, angelegt ist. Deutlich wird zudem, dass beide Figuren bereits eminente Vertreter der deutschen Wissenschaft sind.

Dementsprechend werden die ersten Analepsen in Bezug auf den Status der Figuren in der Gegenwartsebene eingeführt. Zu Humboldt, der auch die letzte auftretende Figur des ersten Kapitels ist, heißt es in Kapitel 2:

Alexander von Humboldt war in ganz Europa berühmt wegen einer Expedition in die Tropen, die er fünfundzwanzig Jahre zuvor unternommen hatte. [...] Er war der jüngere von zwei Brüdern. Ihr Vater, ein wohlhabender Mann von niederem Adel, war früh gestorben. Seine Mutter hatte sich bei niemand anderem als Goethe erkundigt, wie sie ihre Söhne auszubilden habe. ${ }^{573}$

Es findet eine schrittweise vollzogene Rückblende in die Kindheit Humboldts statt. Der Protagonist des Handlungsstrangs wird explizit benannt und aus einer Perspektive eingeführt, die dem bereits bekannten Gegenwartssetting des Jahres 1828 entspricht. Dann wird ein weiterer Schritt zurück in Humboldts Kindheit unternommen, der zu Vater und Mutter und einer ab-ovo-Situation führt. Die Stufen sind deutlich markiert, sodass der Beginn der Analepse leicht nachvollziehbar ist.

Bei der Einführung des ersten Gauß-Kapitels verfolgt Kehlmann eine ähnliche Strategie:

Wer den Professor nach frühen Erinnerungen fragte, bekam zur Antwort, daß es so etwas nicht gebe. [...] Leblos und zweitklassig fühlte sich etwa die Erinnerung an den Nachmittag an, als er seinen Vater beim Abzählen des Lohns korrigiert hatte. [...] Jede andere hatte mit seiner Mutter zu tun. [...] Er liebte sie unsagbar. Er würde

572 Kehlmann 2010 b, S. 7.

573 Ebd., S. 19. 
sterben, stieße ihr etwas zu. Das war keine Redensart. Er wußte, daß er es nicht überleben würde. So war es gewesen, als er drei Jahre alt war [...].574

Kehlmann verwendet mit dem Professorentitel dieselbe Bezeichnung, mit der er Gauß am Romanbeginn einführt. Der Erinnerungstopos eröffnet unmittelbar einen Blick zurück in die Vergangenheit. Kehlmann verweist wie bei Humboldt auf die Eltern des Protagonisten, ehe er mit der Altersangabe eine Einordnung in die frühe Kindheit von Gauß vornimmt. Darüber hinaus zeigt er neben dessen wissenschaftlicher Persona auch dessen menschliche Seite, die es dem nicht-wissenschaftlich-bewanderten Leser erleichtert, eine Beziehung zu der Figur herzustellen.

In der Folge erleichtert das Schema des regelmäßigen Wechsels zwischen der Gauß- und der Humboldt-Erzählung die Orientierung. Zudem erzählt Kehlmann beide Handlungsstränge stringent chronologisch und leitet sie durch thematische Titel ein, die auf das im jeweiligen Kapitel erzählte Abenteuer des jeweiligen Protagonisten verweisen. ${ }^{575} \mathrm{Zu}$ beachten ist der Übergang zwischen der letzten Analepse und dem Sprung auf die Gegenwartsebene. So heißt es am Ende des letzten Humboldt-Kapitels in Bezug auf dessen Rückkehr in die Heimat: 'Nach Berlin? Humboldt lachte. Kein Mensch von Verstand könne diese greuliche Stadt sein Zuhause nennen. Er meine natürlich Paris. In Berlin, soviel sei sicher, werde er nie wieder wohnen.' ${ }^{576}$ Der Beginn des darauffolgenden Kapitels lautet schließlich: 'Mißmutig legte Gauß die Serviette weg. Das Essen hatte ihm gar nicht geschmeckt. Aber da er sich schlecht darüber beschweren konnte, begann er über die Stadt zu schimpfen. Er fragte, wie man es hier aushalten könne. Es habe auch Vorteile, sagte Humboldt. ${ }^{577}$ Kehlmann forciert den Übergang mit der thematischen Verbindung des Unmuts über Berlin. Dabei behält er den Wechsel zwischen der Humboldt- und der Gauß-Erzählung bei, indem zunächst letzterer agiert. Durch die Einführung Humboldts als tatsächlich präsentem Gesprächspartner verdeutlicht Kehlmann den Wechsel auf die Gegenwartsebene, da Humboldt und Gauß nur dann physisch aufeinander treffen.

Das Kapitel 'Die Steppe' birgt eine letzte für Lesbarkeit interessante Erzählstrategie. In dem Kapitel spiegelt sich die Doppelhelix der Erzählstruktur vor dem

\footnotetext{
574 Ebd., S. 53.

575 Bei Humboldt handelt es sich etwa um die wichtigsten Stationen seiner Reise: 'Das Meer', 'Die Höhle', 'Der Fluß', 'Der Berg' und 'Die Hauptstadt'.

576 Kehlmann 2010 b, S. 214.

577 Ebd., S. 215.
} 
Zusammentreffen in Berlin. Humboldt bricht zu einer Expedition nach Russland auf; Gauß forscht in Göttingen. Die Erzählung wechselt ständig zwischen Humboldt und Gauß. Der Übergang wird immer dadurch vorbereitet, dass der jeweils andere zum Ende hin in die Erzählung des einen Eingang findet. Dabei zeichnet sich folgende Entwicklung ab: Zunächst funktionieren die Übergänge über Briefe, gemeinsame wissenschaftliche Fragestellungen, die den einen an die Theorien des anderen erinnern, und über physische Gegenstände wie eine Karte Russlands, die Gauß an Humboldt denken lässt ehe die Erzählung zu Humboldts Expedition changiert. ${ }^{578}$ Dann besteht die Überleitung darin, dass es Humboldt nicht mehr gelingt, an Gauß zu schreiben; schließlich funktioniert sie über ein Traummotiv: Gauß erwacht gegen Ende seines Abschnitts und Humboldt dann zu Beginn des seinen. ${ }^{579}$ Zuletzt entzieht Kehlmann dem Austausch jegliche materielle Substanz und beide kommunizieren durchgehend telepathisch. ${ }^{580}$ Die bekannte Wechselstruktur wird graduell konsequent und für den Leser leicht verständlich zugespitzt.

Durch die Auffächerung in zwei Zeitebenen und die Aufsplitterung in zwei gleichrangige Haupthandlungen gestaltet Kehlmann den Roman zwar komplexer, als es die aristotelische Theorie vorsieht, wirkt dem jedoch durch vereinfachende Mittel entgegen, sodass eine Tendenz zur Mitte sichtbar wird. Zum einen dient den Analepsen die Chronologie der Lebensläufe als roter Faden. ${ }^{581}$ Zum anderen bewirken die Repetition der Wechselstruktur und die leicht nachvollziehbare Gestaltung der Sprünge zwischen Ebenen und Strängen eine einfache Orientierung in der Handlung. Die Analepsen haben dabei eine explikative Funktion für die Rahmenhandlung: sie begründen den wissenschaftlichen Ruhm und das private Scheitern der Protagonisten. ${ }^{582}$

Kehlmann wählt zwei geniale Protagonisten, deren professionelle Errungenschaften für eine breite, nicht fachspezifisch gebildete, Leserschaft nicht leicht

578 Vgl. ebd., S. 266, 268, 271 und 274.

579 Vgl. ebd., S. 280 und 283.

580 Vgl. ebd., S. 290-293. Zudem wurde die Möglichkeit von Telepathie schon zu Beginn des Romans etabliert, vgl. ebd., S. 13.

${ }^{581}$ Im Erzählstrang Humboldts kann zudem das Reisemotiv als roter Faden gelten. Beispielsweise greift Kehlmann im Kapitel 'Der Fluß' auf den laut ihm für die südamerikanische Literatur exemplarischen Erzählgestus eines 'scheinbar unstrukturierten, sprudelnden Erzählens' zurück, das 'ständige Entwickeln und Wegwerfen von kleinen und kleinsten Geschichten, die alle gleich wichtig scheinen und die man achtlos fallen lässt' (Kehlmann $2011 \mathrm{c}, \mathrm{S}$. 165). Auch wenn dies gegen die Vorstellung einer stringenten Handlung spricht, dynamisieren die stets mit Ortswechseln verbundenen Mikroerzählungen Humboldts Reisen, durch deren größeren Kontext sie zusammengehalten werden.

582 Kehlmanns Folgewerke Ruhm (2009) und F (2013), folgen diesem Schema nicht mehr. Bei Ruhm ist dies der in neun Geschichten aufgeteilten Struktur geschuldet, in $F$ sind die Übergänge zwischen den verschiedenen Erzählsituationen weitaus weniger deutlich markiert, sodass der Leser selbst mehr Arbeit leisten muss, um sich die Zusammenhänge zu erschließen. Vgl. Kehlmann 2009 und Kehlmann 2013. 
zugänglich sind. Durch das Interesse an den privaten Mängeln der Figuren, wie etwa Humboldts unterdrückte Pädophilie oder Gauß' Umgang mit seinem Sohn, eröffnet Kehlmann eine 'wechselnde Optik' - im Sinne Thomas Manns - auf die Figuren, um sie auch für ein Nichtfachpublikum interessant zu gestalten. Dabei spielt er mit der Diskrepanz zwischen Privatperson und Genie, was einen weiteren Reiz für ein breites Publikum darstellt. Dies gelingt ihm durch die Figurenkonstellation. Jedem der beiden Protagonisten wird z.B. in Aimé Bonpland bzw. Eugen Gauß ein Begleiter zur Seite gestellt, der die Charakterisierung der Hauptfigur durch Kontrastierung vorantreibt. Neben dem so erzeugten unterhaltenden Humor fungieren die Kontrastfiguren zudem als Helfer, um dem Leser den Zugang zu den Wissenschaftlern zu erleichtern. ${ }^{583}$ Als Beispiel kann die Szene von Gauß' Hochzeitsfeier gelten:

[Gauß] stand auf, schluckte und sagte, er habe nicht erwartet, daß er etwas wie Glück finden würde, und im Grunde glaube er auch jetzt nicht daran. Es komme ihm wie ein Rechenfehler vor, ein Irrtum, von dem er nur hoffe, keiner werde ihn aufdecken. Er nahm wieder Platz und wunderte sich über die fassungslosen Blicke. Leise fragte er Johanna, ob er etwas Falsches gesagt habe. Aber woher denn, antwortete sie. Genau diese Rede habe sie sich immer für ihre Hochzeit erträumt. ${ }^{584}$

In dieser Passage treffen Intellekt und Sozialinkompetenz direkt aufeinander. Kehlmann inszeniert Johanna und die Hochzeitsgesellschaft als Vertreter des Publikums. Gauß' Rede versucht, seine wissenschaftliche Seite mit der privaten zu vereinen. Die Rede ist logisch und nachvollziehbar aufgebaut, verfehlt jedoch die rhetorische Qualität des aptum, was bei der Zuhörerschaft wie auch bei den Lesern Befremden auslöst. Durch die ironische Spiegelung der Rezeption innerhalb des Romans macht sich Kehlmann die eigene Erfahrungswelt der Leser zu Nutze. Um sie zu unterhalten, ergreift er die Partei des Publikums, indem er die soziale Inkompetenz der Figur offen zur Schau stellt und den Lesern eine Anleitung zum Umgang mit der Figur zur Hand gibt. Ebenso kommen die mit Gauß vertrauten Fachleser auf ihre Kosten, indem ihr Erfahrungshorizont ebenfalls Einfluss findet. Die ironische Herangehensweise an ein diffiziles Sujet erinnert an die Methode des 'Zuckerns', in der ein komplexer Sachverhalt unterhaltsam aufbereitet wird, um Breitenwirkung zu generieren.

Aus der Thematik ergibt sich im Hinblick auf die Sprache zunächst Komplexität. An sich greift Kehlmann zumeist auf kurze, einfache Sätze zurück. Da er aber die 
Errungenschaften der beiden Wissenschaftsgrößen abarbeitet, kommt er nicht ohne Fachtermini aus. Dies führt zu einem teils komplexen Wortschatz. Jedoch erklärt Kehlmann Fachwörter explizit; als Beispiel kann die Episode gelten, in der die unterschiedlichen Gerätschaften, die Humboldt zur Vermessungsarbeit nutzt, eingeführt und zugleich erläutert werden. ${ }^{585}$ Eine weitere Verkomplizierung ergibt sich aus der Gestaltung der Figurenrede in indirekter Rede, bei der es sich nicht um eine realistische Darstellungsweise, sondern um eine künstlerische Stilisierung handelt. ${ }^{586} \mathrm{Im}$ Spannungsfeld zwischen Lesbarkeit und literarischer Qualität kann die indirekte Rede als nicht-realistische, komplexe sprachliche Form gelten, die den Lesern eine anspruchsvolle Lektüre verspricht - die sonst einfache Stilistik fungiert als ausgleichender Gegenpol. Aufgrund dieser sowohl bei der sprachlichen Gestaltung als auch allgemein verfolgten dynamischen Strategie, die künstlerische Ambition und Lesbarkeit erfolgreich austariert und dadurch eine breite Leserschaft anspricht, kann die Vermessung als praktische Umsetzung des Lesbarkeitsbegriffs gelten.

\section{Thomas Glavinic}

Die Analyse von Glavinics Autorpoetik wird aufzeigen, dass für ihn Lesbarkeit eine selbstverständliche poetologische Kategorie darstellt. Dementsprechend bemüht er sich in Das größere Wunder intensiv um traditionelle Elemente des U-Pols, die er in seinem qualitativ ambitionierten Roman offensiv ausstellt. Die dazu herangezogenen Methoden sollen durch eine vergleichende Lektüre zu Thomas Manns Roman Der Zauberberg (1924) verdeutlicht werden, der als Folie dient.

In der ZDF-Literatursendung Das blaue Sofa erhebt Glavinic das Erzählen zum zentralen Begriff seines literarischen Projekts: 'Ich bin so auf Erzählen eingestellt. Also mein ganzes Programm, mein Modus heißt Erzählen.' ${ }^{587}$ Das Erzählen bedeutet für Glavinic in Gollners Interviewband eine Abbildung der Wirklichkeit: 'In erster Linie möchte ich einen Teil von Wirklichkeit transportieren, der mich interessiert. Das ist

\footnotetext{
585 'Zwei Barometer für den Luftdruck, ein Hypsometer zur Messung des Wassersiedepunktes, ein Theodolit für die Landvermessung [...]', vgl. ebd., S.37f.

586 Im Roman thematisiert Kehlmann den Realismus selbstreflexiv, indem Humboldt ihm ein Primat innerhalb der Kunst zuerkennt, weil er das Publikum nicht durch unnötige künstlerische Abstraktion irritiert: 'Künstler vergäßen zu leicht ihre Aufgabe: das Vorzeigen dessen, was sei. Künstler hielten Abweichungen für eine Stärke, aber Erfundenes verwirre die Menschen, Stilisierung verfälsche die Welt' (Ebd., S. 221).

587 Herles 2013 b.
} 
immer etwas recht klar Vermittelbares. ${ }^{588}$ Neben seiner Vorliebe für eine realistische Gestaltungsweise etabliert Glavinic durch die perspicuitas zugleich deren Affinität zu einer einfach zu durchschauenden Textgestalt.

In seiner Rezension zum Werk des österreichischen Autors Michael Köhlmeier (1999) erkennt Glavinic eine Abneigung gegenüber erzählender Literatur: 'Du tust ja nie etwas anderes als Geschichten erzählen, deswegen mögen dich die Experimentalhansis auch nicht.' 589 Ebenso wie Krausser eröffnet Glavinic eine Dichotomie zwischen Erzählen und Experiment, wobei die Originalität als Qualitätsparameter fungiert. Glavinic hingegen favorisiert 'Geschichten, [...] nicht [tiefsinnige], einfache, unterhaltsame, leicht zu lesende Geschichten eben'.590 Die angeführten Aspekte entstammen dem Lesbarkeitsbegriff: das stringente Erzählen, das vom Autor herbeizuführende leichte Textverständnis sowie das Unterhaltungsdesiderat. Das traditionelle Qualitätsmerkmal der Tiefe spielt keine Rolle.

In der Zeitschrift Spectrum (1999) setzt sich Glavinic dezidiert mit der Qualität des Erzählens auseinander: 'Geschichten erzählen kann Kunst sein, es kommt eben darauf an, ob es dem Autor gelingt, eine interessante Geschichte auf hohem handwerklichen und intellektuellen Niveau spannend zu erzählen.' ${ }^{591}$ Glavinic etabliert eine narrative Literatur als künstlerisch anspruchsvolle Form, wobei er nicht von einem generellen Kunstcharakter ausgeht, sondern gewisse ästhetische Qualitäten geltend macht, die das Erzählen zur Kunstform erheben. Das Intellektuelle findet sich in der Form von hoher Bildung des Autors und der nicht-didaktischen Verarbeitung von Bildungsgütern wie auch bei Krausser und Kehlmann als Qualitätsindikator. Spannung verweist auf die Unterhaltung des Lesers. In Gollners Interviewband beschreibt Glavinic das Handwerk genauer: 'Eine Geschichte funktioniert nicht ohne Ordnung und Regeln. Kunst ist in diesem Sinn etwas Konservatives. ${ }^{992}$ Unter 'Ordnung' versteht Glavinic wie Kehlmann eine aristotelische Folgerichtigkeit der Handlung, die Regeln entsprechen prinzipiell denen der Wirklichkeit. Im Falter (2011) äußert Glavinic jedoch Einwände gegen eine strikte Wirklichkeitsabbildung: 'Mit dem Wunsch nach totaler Authentizität im Sinne von blanker Abbildung der Realität kann ich nichts anfangen. Wahrhaftigkeit ist der

\footnotetext{
588 Gollner 2005, S. 21.

589 Glavinic 1999 b.

590 Ebd.

591 Glavinic 1999 a.

592 Gollner 2005, S. 22.
} 
Begriff, der mich in der Literatur interessiert. ${ }^{593}$ Glavinic rekurriert wie etwa Fontane, Kästner, Kehlmann und Krausser auf die Distinktion zwischen Wirklichem und Wahrem, wobei er ebenso dem Wahrem eine privilegierte Stellung einräumt und so für sich einen Qualitätsanspruch formuliert.

In seinen Bamberger Poetikvorlesungen Meine Schreibmaschine und ich (2012) artikuliert Glavinic seine Auffassung von einer hochwertigen stilistischen Gestaltung: ' $\mathrm{Zu}$ einem guten Schriftsteller macht einen Schnörkellosigkeit. Ich mag Schnörkellosigkeit. Ich mag keine Großspurigkeit. Und oh, wie sehr ich all diese misslingenden, auftrumpfenden Bemühungen um Ästhetik hasse [...]. Kunst prahlt nicht. ${ }^{594}$ Diese mit Krausser geteilte Abneigung gegen den übermäßigen Einsatz von Stilmitteln entspricht der von Aristoteles angeführten Warnung vor übermäßiger Stilisierung, die das Gleichgewicht zwischen Simplizität und Komplexität breche und einen Text unverständlich mache. Ein weiterer stilistischer Vorsatz ist der einer möglichst großen 'Genauigkeit' ${ }^{595}$ Der an die kästner'sche 'Präzisionsästhetik' und Kehlmanns Fokus auf Details erinnernde Terminus steht für eine anspruchsvolle Auseinandersetzung mit der brevitas, die sich eine leichte Verständlichkeit zum Ziel setzt.

Verständlichkeit ist in Glavinics Vorlesungen nicht nur bei der Stilistik von zentraler Bedeutung: 'Ich mag Bücher, die leichtfüßig von der Schwere erzählen. Ich mag vielschichtige Erzählebenen. Ich mag Bücher, die oberflächlicher wirken, als sie sind. ${ }^{596}$ Wie Kehlmann und Krausser etabliert Glavinic eine leicht lesbare Verarbeitung eines anspruchsvollen Sujets als Qualität. Prägnant ist der Fokus auf Oberfläche, Tiefe und Erzählebenen, der sich auch bei Kehlmann und Krausser findet und den Glavinic in dem programmatischen Essay 'Das Handwerk des Romans' (2008) an intertextuelle Verweise knüpft: 'Für das umfassende Verständnis des Romans spielt es tatsächlich keine Rolle, ob man als Kind Astrid Lindgrens Michel gelesen hat oder nicht. Wenn nicht, merkt man nichts. Wenn ja, sickert etwas in den Hinterkopf.' ${ }^{597}$ Dies ist die postmoderne Technik des ‘esoterischen’ und ‘exoterischen' Vergnügens: Der allgemeine Leser zieht aus der leicht verständlichen Handlung Vergnügen. Der literarisch

\footnotetext{
593 Schaffer 2011, S. 53.

594 Glavinic 2014 a, S. 42.

595 Vgl. ebd., S. $56 f$.

596 Ebd., S. 28.

597 Glavinic 2014 b.
} 
bewanderte Leser zieht es aus dem Erkennen der intertextuellen Referenz. Dadurch kann die Leichtigkeit des Erzählten bewahrt und eine breite Leserschaft unterhalten werden.

Hinsichtlich der Breitenwirkung äußert Glavinic im Falter: 'Ich hoffe, ich gehöre zu jenen Autoren, die Kunst machen und trotzdem gelesen werden. Und zwar nicht nur von drei- oder fünftausend Leuten. ${ }^{598}$ Wie Adorno und Horkheimer unterscheidet Glavinic zwischen einer künstlerisch wertvollen und einer breitenwirksamen Literatur, die für ihn jedoch keine unvereinbaren Pole darstellen. Er strebt eine Synthese an, die ihm sowohl künstlerische Anerkennung als auch Breitenwirkung sichert. Glavinic äußert sich gegenüber Die Presse (2014) dezidiert zum Markterfolg:

Solange ich zu Hause an meinem Buch arbeite, produziere ich ein Kunstwerk. Aber sobald ich es dem Verlag schicke und der es vervielfältigt, wird eine Ware daraus. Verlag und Autor sind daran interessiert, möglichst viele Exemplare zu verkaufen. Davon lebt ein Schriftsteller. Es können ja nicht nur reiche Adelige Bücher schreiben. ${ }^{599}$

Glavinic differenziert zwischen dem Status eines Buchs während der Genese und danach. Beim Schreiben zählen für ihn lediglich künstlerische Aspekte. Nach der Veröffentlichung sind hohe Verkaufszahlen Desiderat, wobei er es akzeptiert, Literatur generell als Verkaufsware anzusehen. Zuletzt spielt Glavinic ironisch auf die im 18. Jahrhundert vom Markt abgelöste höfische Literaturproduktion an, die nicht auf finanziellen Verdienst aus war, und rechnet somit das Geldverdienen als selbstverständliche Funktion der schriftstellerischen Tätigkeit zu. Außerdem verbindet Glavinic wie Wieland finanziellen Erfolg mit der Qualitätssicherung. Während Wieland mit hohen Summen die besten Autoren für seinen Teutschen Merkur zu gewinnen gedenkt, konstatiert Glavinic, dass 'Künstler auch bessere Arbeit leisten, wenn es ihnen materiell gut geht und sie ihre Bilder nicht unter der Brücke malen oder ihre Romane nicht im ungeheizten Kellerabteil schreiben müssen'. ${ }^{600}$ Glavinic sieht die Schaffung eines angenehmen und von existenziellen Sorgen freien Lebens- und Arbeitsraums als grundlegende Voraussetzung qualitativ ansprechender Literatur. Darüber hinaus formuliert er im Presse-Interview den Wunsch nach Bestsellerdimensionen. Auf die

\footnotetext{
598 Nüchtern 2007.

599 Hierländer/Stern 2014.

600 Anon. 2013.
} 
Frage, ob er neidisch auf seinen Freund Daniel sei, entgegnet er, dass er auch 'gern zwei Millionen Bücher verkaufen würde. ${ }^{901}$ Glavinic sieht demnach nicht nur wie Krausser finanzielle Absicherung als erstrebenswert an, sondern auch den großen Verkaufserfolg. Künstlerische Qualität ist für ihn mit finanziellem Erfolg vereinbar und auch erstrebenswert, um ebenjene Qualität zu sichern.

In seinen Poetikvorlesungen formuliert Glavinic ein Streben nach internationaler Breitenwirkung: '[Ich] wollte niemals ein österreichischer Autor sein, ich wollte jemand sein, der bedeutende Romane für die ganze Welt schreibt und eben zufällig in Österreich geboren ist. ${ }^{602}$ Wie Krausser und Kehlmann sucht Glavinic vehement den Anschluss an den internationalen Literaturbetrieb. Dazu entwirft er in Meine Schreibmaschine und ich ein ähnliches Szenario wie Krausser im Text+Kritik-Essay zu Kehlmann:

Es gibt Menschen, die sich insgeheim zu Tode langweilen bei einem Buch, es dann aber loben und dessen Autor mit Preisen überhäufen. [...] Und dann wundern sich dieselben Leute im Feuilleton, warum deutsche Literatur kaum übersetzt und im Ausland nur wahrgenommen wird, wenn ein alter Herr wieder mal ein seltsames politisches Gedicht veröffentlicht. ${ }^{603}$

Glavinic beklagt den Umstand, dass die Kritik Werke fördert, die nicht auf die Unterhaltung der Leser zielen. Wie Krausser führt er darauf den Misserfolg der deutschen Literatur im unterhaltungsaffinen Ausland zurück und benennt Unterhaltung als Mittel zur Erschließung des internationalen Marktes. Das Übersetztwerden avanciert zum Anspruch. Schließlich offenbart Glavinic ein Unbehagen gegenüber einer politisch engagierten Literatur, hier gegenüber dem Israel-Gedicht von Günter Grass, womit er ebenso eine auf die ältere Generation fokussierte Fehlrepräsentation der deutschsprachigen Literatur im Ausland suggeriert.

Im Interview mit der Presse am Sonntag (2009) lehnt Glavinic einen politischen Aktivismus in der Literatur ab:

Es gibt mit Schreiben eine Sache, von der ich annehme, dass ich sie recht gut kann. Aber das bedeutet noch nicht, dass ich automatisch mehr von Politik verstehe als andere. Ich möchte nicht zu jenen gehören, die sich zu allem und jedem zu Worte melden $[\ldots] .604$

\footnotetext{
601 Hierländer/Stern 2014.

602 Glavinic 2014 a, S. 47.

603 Ebd., S. 103.

604 Steiner 2009.
} 
Wie Krausser und Kehlmann wendet er sich gegen die Vorstellung, dass Autoren eine größere politische Kompetenz als andere Gesellschaftsgruppierungen hätten, weswegen er auf sämtliche Stellungnahmen verzichtet. In seinen Poetikvorlesungen erweitert er dies auf nicht-politisch motivierte didaktische Funktionen: 'Ich mag Ideologie nicht. Ich mag keine Literatur, die sich in den Dienst einer Sache oder einer Meinung stellt [...]. Ein Roman ist nur sich selbst verpflichtet, das sollte mittlerweile allgemein bekannt sein. ${ }^{605}$ Glavinic impliziert eine inzwischen vollzogene Abkehr von jedweder Art der ideologischen Vereinnahmung von Literatur. Dies bestätigt die im Literaturstreit initiierte Abkehr von einer 'Gesinnungsästhetik'. Glavinic löst den Roman von didaktischen Pflichten und propagiert die Kunstauffassung des l'art pour l'art. ${ }^{606}$

Die Wirkungsabsicht, für die Glavinic in seiner Köhlmeier-Rezension explizit eintritt, ist Unterhaltung: 'Unterhaltung, sagen manche, habe nichts mit Kunst zu tun, und beweisen damit, daß sie von Literaturgeschichte keine Ahnung haben.' ${ }^{607}$ Glavinics Aussage kann alls Rückgriff auf die Rezeption Adornos und Horkheimers gelesen werden, in der sich Unterhaltung und Kunst gegenseitig ausschließen. Um seine eigene Position zu legitimieren, beruft er sich auf die umfassendere Tradition der Literaturgeschichte und vertritt dabei wie Böll und Krausser die Ansicht, dass 'wahre' bzw. 'große' Literatur auch zwingend große Unterhaltung zu sein habe. Dadurch etabliert Glavinic Unterhaltung als selbstverständliche Qualität.

Wie aus dem Fazit von Glavinics Poetikvorlesungen hervorgeht, macht dieses Unterhaltungsdesiderat kombiniert mit Lesbarkeit den Kern seiner Poetik aus: 'Wenn ich an einer Stelle zwei gleichwertige Möglichkeiten habe, sei es sprachlich, sei es strukturell, sei es inhaltlich, dann wähle ich immer die anschaulichere, konkretere, ja unterhaltsamere. Unterhaltung ist nichts Böses, wenn sie klug ist.' ${ }^{608}$ Glavinic privilegiert Lesbarkeit und Unterhaltung gegenüber komplexeren Formen. Dabei führt er die Anschaulichkeit an, die das Hauptziel der antiken Rhetorik ist und für ein leichtes Textverständnis steht. Wie Wittstock lässt er Unterhaltung nicht per se als Qualität gelten, sondern beruft sich bei deren Ausgestaltung auf eine qualitative Ambition. Wie für Krausser und Kehlmann gehen Lesbarkeit und Unterhaltung mit literarischer

\footnotetext{
605 Glavinic 2014 a, S. 36.

606 Dass sich Glavinic in seinem Werk nicht strikt daran hält, zeigt sein Roman Lisa (2011), in dem sich ein IchErzähler, der sich bezeichnenderweise als Tom vorstellt, in hauptsächlich politischen Schimpftiraden ergeht; vgl. Glavinic 2013 c.

607 Glavinic 1999 b.

608 Glavinic 2014 a, S. 114.
} 
Qualität einher, wodurch Glavinic die Vorurteile gegenüber einer publikumsfreundlichen Literatur, die auf eine breite Leserschaft im In- und Ausland zielt, widerlegt und deren künstlerischen Gehalt herausstellt.

Das größere Wunder erinnert nicht nur aufgrund seines Hochgebirgssujets an Manns Zauberberg, sondern weist auch in seiner Figurenkonstellation Ähnlichkeiten mit Manns Sanatoriumsroman auf. Sowohl bei Glavinics Jonas als auch bei Manns Hans Castorp handelt es sich um finanziell wohlsituierte Waisen, die eine starke Bindung zu ihren Großvätern, bzw. im Falle von Jonas zu seinem Adoptivgroßvater, aufbauen. Zudem werden ihnen im Adoptivbruder Werner und dem Cousin Joachim Ziemßen zwei enge Bezugspersonen zur Seite gestellt - mit dem Unterschied, dass Jonas zunächst noch einen Zwillingsbruder hat.

In Bezug auf Werner und Joachim ergeben sich weitere Übereinstimmungen: beide sterben früh und werden dann aus dem Totenreich zurückgerufen. Bei Mann geschieht dies im Zuge einer spiritistischen Séance; Castorp wagt es nicht ihn anzusprechen und löst die Versammlung auf. ${ }^{609}$ Glavinic setzt sich intensiver mit magischen Elementen und transzendenten Erscheinungen auseinander. Jonas und Werner sind im Leben telepathisch miteinander verbunden und können nonverbal kommunizieren. ${ }^{610}$ Sie schließen einen Pakt, dass derjenige, der früher stirbt, dem anderen in einem Moment, in dem der andere dringend Hilfe benötigt, ein Zeichen gibt. ${ }^{611}$ Dies geschieht als Jonas versucht, vom Everest abzusteigen. Dabei kommt es zu einem Wettstreit zwischen Werners Erscheinung, die Jonas retten und zum Abstieg bewegen will, und zwei Leichen, die ihn zum Rasten und Sterben verleiten wollen. ${ }^{612}$ Wie Mann nutzt Glavinic das Höhensetting, um übernatürliche Phänomene zu etablieren, wobei sich diese bei Glavinic nicht allein auf die Höhe beschränken.

Hinsichtlich der Erzählstruktur finden sich zwischen den beiden Romanen ebenfalls Korrespondenzen. Beide beginnen mit der Ankunft des Protagonisten am Berg und führen dann durch Analepsen dessen Kindheit ein. Während Mann die Rückblenden sporadisch, aber in eindeutig abgegrenzten Kapiteln, einstreut, sind diese für Glavinics

\footnotetext{
609 Vgl. Mann 2013, S. 922-938; auf diese Szene verweist auch Kehlmanns Vermessung. Während einer Séance spricht ein Medium zu Humboldts Mutter, vgl. Kehlmann 2010 b, S. 253-255.

610 Im Zauberberg hat das Medium Elly Brand die Fähigkeit zur Telepathie; vgl. Mann 2013, S. $902 \mathrm{f}$.

611 Vgl. Glavinic 2013 a, S. 39-41.

612 Diese Szene erinnert an die Chimborazo-Episode in Kehlmanns Vermessung, in der Humboldt und Bonpland in der Höhe andere Personen bzw. andere Versionen von sich selbst herbeihalluzinieren, vgl. Kehlmann 2010 b, S. $163-180$.
} 
Roman konstitutiv. ${ }^{613}$ Glavinic spannt ähnlich der Vermessung eine Doppelhelix auf, die erst im 51. Kapitel endet. ${ }^{614}$ Dabei fungieren die Kapitel mit ungeraden Nummern als Gegenwartsebene, in der Jonas den Everest besteigt; die Kapitel mit geraden Nummern setzen in Jonas' Kindheit ein. ${ }^{615}$ Die Reihenfolge der Analepsen sowie die Erzählungen der einzelnen Rückblenden und der gegenwärtigen Zeitebene sind, wie im Zauberberg, chronologisch gehalten. Somit entsteht durch die Auffächerung in die unterschiedlichen Ebenen zwar eine größere Komplexität, allerdings garantieren die Chronologie der Handlungsführung und die Regelmäßigkeit der Abwechslung einen leichten Zugang zum Erzählten. Außerdem ist der Übergang vom stetigen Wechsel der Ebenen zum durchgängigen Erzählen auf der Gegenwartsebene leicht nachvollziehbar:

Von Oslo flog [Jonas] nach London. Im Fabric traf er Helen wieder, mit der er vor vielen Jahren ein paar Mal nach Hause gegangen war, und unterschrieb am nächsten Morgen an ihrem Küchentisch den Vertrag für den Everest, ohne auch nur eine Sekunde nachzudenken. ${ }^{616}$

Diese Stelle verlinkt die Analepsen durch Abschluss und Anschluss zweier thematischer Einheiten mit Kapitel 1 und dem Beginn der gegenwärtigen Erzählebene: zum einen durch den vertraglichen Beschluss, den Everest zu besteigen, zum anderen durch die Auflösung der Helen-Geschichte. In Bezug auf Helen ergeben sich zwei Funktionen. Erstens fungiert die zitierte Stelle explanatorisch, da sie eine Erklärung der seit Kapitel 7 ständig angestellten Vermutung über Jonas' Verhältnis zu Helen liefert. ${ }^{617}$ Die bis dato aufgebaute Spannung beim Leser wird aufgelöst. Zweitens bildet die Konstellation Helen-Jonas einen unmittelbaren Anschluss an Kapitel 1, in dem Helen die erste Person ist, mit der Jonas interagiert. ${ }^{618}$ So kommt eine Art dramatische Personenkette zustande, die dem Leser einen Bezugspunkt zwischen den beiden Handlungsebenen liefert und ihm so den Übergang erleichtert.

Der Entschluss, zum Everest aufzubrechen, schließt chronologisch direkt an das erste Kapitel an, in dem Jonas im Basiscamp eintrifft. Zudem wird der Everest im ersten

\footnotetext{
613 Beispielsweise lautet der Titel des ersten Kindheitskapitels im Zauberberg 'Von der Taufschale und vom Großvater in zwiefacher Gestalt'. Das Taufbild verweist unmittelbar auf ein Erzählen $a b$ ovo und erleichtert dem Leser die Orientierung; vgl. Mann 2013, S. 32.

614 Vgl. Glavinic 2013 a, S. 444; Kehlmann springt jedoch in seiner Doppelhelix nicht zwischen den unterschiedlichen Erzählebenen, sondern zwischen den Protagonisten Gauß und Humboldt.

615 Vgl. ebd., S. 7 und 12.

616 Ebd., S. 443.

617 Vgl. ebd., S. 67.

618 Vgl. ebd., S. 9-11.
} 
Kapitel als zentraler Eckpunkt von Jonas' Entwicklung etabliert. Der Everest sei jener Berggigant, 'der ihn schon als Kind auf geradezu unheimliche Art fasziniert hatte [...]. Er dachte an den Weg, der ihn hierher geführt, der ihn um die Welt getrieben hatte, der ihn in Südamerika auf Marie hatte stoßen lassen [...].' ${ }^{619}$ Der Erzähler gibt dem Leser, der sich zu diesem Zeitpunkt der Analepsen noch nicht bewusst ist, ein Programm für das Sujet und die Struktur der Rückblenden vor, an deren Anfang und Ende der Everest steht. Dadurch wird die Spaltung in Rahmen und Binnenhandlung vorweggenommen, was den Leser auf den tatsächlichen Wechsel vorbereitet. Die Binnenhandlung - Jonas' Weg zum Everest - hat die explikative Funktion, seine Motivation zum Aufstieg plausibel zu machen. In Kombination mit der Einführung der Figur Marie, die Jonas, wie sich später herausstellt, durch das Bezwingen des Berges zurückerobern will, erweckt dies bei den Lesern Neugier und Spannung. Trotz der Zeitsprünge kreiert Glavinic durch folgerichtige Einbettung, Wiederholung und eine implizite Lektüreanleitung eine anschauliche Erzählung, die es einer breiten Leserschaft ermöglicht, sich in den unterschiedlichen zeitlichen und örtlichen Gegebenheiten zurechtzufinden.

Allerdings zieht Glavinic eine weitere Ebene ein, die speziell auf diejenigen Leser zielt, die mit seinem Werk vertraut sind. Die Figurenkonstellation Jonas (Held), Werner (bester Freund) und Marie (Jonas' Freundin) ist aus den Romanen Die Arbeit der Nacht und Das Leben der Wünsche (2009) bekannt. Das Feuilleton sprach daher bei der Veröffentlichung von Das größere Wunder des öfteren von einer 'Jonas-Trilogie' ${ }^{620}$ Die ersten beiden Bücher verhandeln Szenarios, die nicht mit einer realistischen Grundhaltung zu vereinbaren sind, sondern zwischen dem réalismo magico und den Werken Franz Kafkas anzusiedeln sind: Was passiert, wenn plötzlich alle Menschen von der Erde verschwinden und ich als einziger übrig bin, und was passiert, wenn ich plötzlich drei Wünsche frei habe. In Das größere Wunder baut Glavinic Stellen ein, die auf den direkten Vorgänger, Das Leben der Wünsche, verweisen. Am deutlichsten wird dies an zwei Stellen. Zum einen trifft Jonas einen Jungen, der auf die Frage, was er sich wünschen würde, wenn er drei Wünschen frei hätte, antwortet, dass er sich mehr Wünsche wünschen würde. ${ }^{621}$ Dies entspricht dem Vorgehen der Jonas-Figur aus Das

\footnotetext{
619 Ebd., S. 8.

620 Vgl. u.a. Gösweiner 2013, Strigl 2013, Wiele 2013, Hugendick 2013.

621 Glavinic 2013 a, S. 381.
} 
Leben der Wünsche. ${ }^{622}$ Die zweite klare Referenz wird im Laufe des Romans aufgebaut. An mehreren Stellen wird Jonas von Träumen heimgesucht, in denen eine riesige Welle auf ihn zukommt. ${ }^{623}$ Dies kulminiert in einem leicht variierten Zitat des Endes von Das Leben der Wünsche:

Er stand mit einer gesichtlosen Frau am Strand einer einsamen Insel, als sich am Horizont ein ungeheurer Tsunami erhob. Die Welle rollte heran, kam näher und näher, erstarrte plötzlich. Die Frau beugte sich zu ihm hinüber und flüsterte ihm etwas ins Ohr, das er nicht verstand. ${ }^{624}$

Zum einen funktioniert das Meer als Kontrastsystem zum Hochgebirge. Ebenso wie später den Berg versucht Jonas surfend die Wellen zu bezwingen; dass Gebirge und Meer in dieser Hinsicht in einen Kontext gesetzt werden, zeigt auch Maries in beiden Fällen vorgetragene Bitte, Jonas möge sich in ihrer Gegenwart nicht umbringen. ${ }^{625}$ Der Leser kann ohne Probleme die Bezüge herstellen und den Handlungsfortgang leicht nachvollziehen. Dann erkennt der literarisch kundige Leser eine weitere Übereinstimmung mit Manns Zauberberg. Nicht nur stammt Castorp aus Hamburg und übt den Beruf des Schiffbauers aus, sondern sehnt sich im Kapitel 'Strandspaziergang' ans Meer. ${ }^{626}$ Die Herstellung des Zusammenhangs zwischen Glavinic und Mann bei der literarischen Ausgestaltungen der Meer-Berg-Opposition stellt ein 'esoterisches' Vergnügen dar. ${ }^{627}$ Dies gilt auch für die Glavinic-Kenner. Glavinic baut die Referenzen auf Das Leben der Wünsche als Entschlüsselungsangebot ein, um jenen durch das Erkennen des intertextuellen Spiels Vergnügen zu bereiten. ${ }^{628}$ Ziel ist es, möglichst viele Leser zu unterhalten, ohne sie beim Verfolgen der Handlung vor Schwierigkeiten zu stellen.

\footnotetext{
622 Vgl. Glavinic 2011, S. 14.

623 Vgl. Glavinic 2013 a, S. 214f, $231 \mathrm{f}$ und 242.

${ }^{624}$ Ebd., S. 361; in Das Leben der Wünsche lautet die Stelle: 'Eine unaufhörlich wachsende Wand kam. Was da auf ihn zurollte, hatte kein menschliches Auge vor ihm gesehen. Marie bewegte die Lippen, aber er hörte sie nicht. Er war taub. [...] Für diese eine Sekunde stand die Wasserwand vor ihm wie ein Bild.' (Glavinic 2011, S. 317).

625 Vgl. Glavinic 2013 a, S. 385f, 402-404 und 516.

626 Vgl. Mann 2013, S. 741-750.

627 Generell operiert Glavinic mit Referenzen aus der E- und der U-Literatur, was Leslie Fiedlers Forderung nach der Überwindung der Grenzen und auch Kraussers poetologischen Äußerungen entspricht. Glavinic etwa rekurriert früh im Roman auf Brechts berühmtes Diktum vom Fressen und der Moral aus der Dreigroschenoper, vgl. Glavinic 2013 a, S. 73. Im Laufe der Erzählung verweist er dann z.B. gleichermaßen auf Andrew Lloyd Webbers Musical Cats, vgl. ebd., S. 60 und $430 \mathrm{f}$.

628 Eine umfassende Gesamtanalyse der Trilogie und der Beziehungen zwischen den Romanen ist noch zu leisten. Zudem ist diese Werkentwicklung noch nicht abgeschlossen, da im Frühjahr 2016 mit Der Jonas-Komplex ein vierter Jonas-Roman erscheint.
} 
Wie Glavinic seinen Roman möglichst lesbar gestaltet, lässt sich anschaulich anhand eines direkten Textstellenvergleichs mit einer Passage aus dem Zauberberg aufzeigen. Sowohl Glavinics Jonas als auch Manns Castorp sind Sinnsuchende. So werden in beiden Romanen philosophische Fragestellungen erörtert, die sich beispielsweise bei den Themen Liebe, Tod und Zeit überschneiden. Während Mann Castorp zwischen den intellektuellen Schwergewichten Settembrini und Naphta positioniert, die sich in komplexen und mit Bildungsgut gespickten Dialogen ergehen und Castorp stellvertretend für den Leser belehren, nutzt Glavinic die realistische Herangehensweise, um trotz des diffizilen Sujets leicht zugänglich schreiben zu können. Jonas’ Bezugspunkt ist vor allem sein Adoptivgroßvater Picco.

Als exemplarisch für die Komplexität der intellektuellen Dispute im Zauberberg können Naphtas Ausführungen zum Streit zwischen Naturwissenschaft und Kirche um die Deutungshoheit gelten:

'Wahr ist, was dem Menschen frommt. In ihm ist die Natur zusammengefaßt, in aller Natur ist nur er geschaffen und alle Natur nur für ihn. Er ist das Maß der Dinge und sein Heil das Kriterium der Wahrheit. [...] Die christlichen Jahrhunderte waren völlig einig über die menschliche Unerheblichkeit der Naturwissenschaft. Lactantius, den Konstantin der Große zum Lehrer seines Sohnes wählte, fragte geradeheraus, welche Seligkeit er denn gewinnen werde, wenn er wisse, wo der Nil entspringt, oder was die Physiker vom Himmel faseln. [...] Wenn man die platonische Philosophie jeder anderen vorzog, so darum, weil sie sich nicht mit Naturerkenntnis, sondern mit der Erkenntnis Gottes abgab. Ich kann Sie versichern, die Menschheit ist im Begriff, zu diesem Gesichtspunkt zurückzufinden und einzusehen, daß es nicht Aufgabe wahrer Wissenschaft ist, heillosen Erkenntnissen nachzulaufen, sondern das Schädliche oder auch nur ideell Bedeutungslose grundsätzlich auszuscheiden und, mit einem Worte, Instinkt, Maß, Wahl zu bekunden. Es ist kindisch, zu meinen, die Kirche habe die Finsternis gegen das Licht verteidigt. Sie tat dreimal wohl daran, ein "voraussetzungsloses" Streben nach Erkenntnis der Dinge, das heißt: ein solches, das sich der Rücksicht auf das Geistige, auf den Zweck der Heilserwerbung entschlägt, für strafbar zu erklären, was den Menschen in Finsternis geführt hat und immer tiefer führen wird, ist vielmehr die "voraussetzungslose", die aphilosophische Naturwissenschaft.'[...]

'Ich bringe ein wenig Logik in Vorschlag', versetzte Naphta. 'Entweder Ptolemäus und die Scholastik behalten recht, und die Welt ist endlich in Zeit und Raum. Dann ist die Gottheit transzendent, der Gegensatz von Gott und Welt bleibt aufrecht, und auch der Mensch ist eine dualistische Existenz: das Problem seiner Seele besteht in dem Widerstreit des Sinnlichen und des Übersinnlichen, und alles Gesellschaftliche ist mit Abstand zweiten Ranges. Nur diesen Individualismus kann ich als konsequent anerkennen. Oder aber Ihre Renaissance-Astronomen fanden die Wahrheit, und der Kosmos ist unendlich. Dann gibt es keine übersinnliche Welt, keinen Dualismus; das Jenseits ist ins Diesseits aufgenommen, der Gegensatz von Gott und Natur hinfällig, und da in diesem Falle auch die menschliche Persönlichkeit nicht mehr Kriegsschauplatz zweier feindlicher Prinzipien, sondern harmonisch, sondern einheitlich ist, so beruht der innermenschliche Konflikt 
lediglich auf dem der Einzel- und der gesamtheitlichen Interessen, und der Zweck des Staates wird, wie es gut heidnisch ist, zum Gesetz des Sittlichen. [...]'629

Ein umfassendes Verständnis der Stelle verlangt eine umfassende Bildung des Lesers. Zu Beginn zitiert Mann implizit Protagoras' sophistischen Lehrsatz der Homo-Mensura. Dann werden weitere Philosophen, Theologen und Schulen wie Laktanz, Platon, die Logik, Ptolemäus und die Scholastik explizit benannt. Außerdem erscheint mit Konstantin eine historisch verbürgte Figur. Völlig vage bleibt, welche drei Verteidigungsmaßnahmen der Kirche gegen die Naturwissenschaften Naphta in Betracht zieht. Zur Klärung dieser Frage wäre ein fundiertes Wissen der Kirchengeschichte von Nöten. Um die Anspielungen entschlüsseln und die philosophischen, theologischen und historischen Gedankengänge nachvollziehen zu können, setzt der Autor beim Leser ein hohes Maß an Bildung voraus, gibt ihm wie z.B. in der Passage zu Ptolemäus und der Scholastik nur wenige erklärende Aspekte. Diese sind jedoch so formuliert, dass sie mit den Termini 'transzendent', 'dualistisch' und 'Individualismus' weitere semantisch komplexe Kategorien einführen, die weitere intellektuelle Fragestellungen nach sich ziehen und die Lektüre für den Leser zusätzlich erschweren. Zudem trägt die komplexe Matrix der Sätze zum schwierigen Verständnis bei. Als Beispiel kann der Satz von 'Ich kann Sie versichern' bis 'Worte, Instinkt, Maß, Wahl zu bekunden' angesehen werden, der hypotaktisch komplex aufgefächert ist. Neben der verhandelten Thematik zeigt auch die Formulierung 'Ich kann Sie versichern', dass die Lesbarkeitsdebatte stets an einen temporalen Aspekt gebunden ist. Rein linguistisch gesehen ist Manns Satzanfang nach der gegenwärtigen Grammatik in diesem Kontext nicht korrekt und widerspricht dem Stilprinzip der Sprachrichtigkeit. Darüber hinaus scheint der Wettkampf um den Hoheitsanspruch zwischen Religion und Wissenschaft inzwischen eindeutig zu Gunsten der Wissenschaft entschieden worden zu sein. So erscheint die Passage zwar als ansprechendes Bild der intellektuellen Diskussion des frühen 20. Jahrhunderts, spiegelt aber nicht die Umstände der Gegenwart. ${ }^{630}$

Glavinic hingegen nutzt eine realistische Gestaltungsweise, um komplexe Fragestellungen lesbar verhandeln zu können. Jonas denkt an eine Unterhaltung mit Picco zurück:

\footnotetext{
629 Mann 2013, S. 546f.

630 Dies gilt auch für die in der Zeit vor dem Ersten Weltkrieg angesiedelten Diskussionen über die politischen Entwicklungen in Europa sowie für alle medizinischen Fachdebatten.
} 
Einmal, er mochte zehn gewesen sein oder elf, hatte ihn Picco beiseitegenommen und gefragt, ob er schon etwas über die Welt wisse.

'Ich weiß viel!' hatte Jonas beleidigt geantwortet.

'Na? Und was weißt du?'

'Man muss zur Schule gehen. Man muss nett zu anderen Leuten sein. Man muss baden und Zähne putzen. Man...'

'Solchen Schnickschnak meine ich nicht', unterbrach ihn Picco. 'Ich meine etwas Tieferes. Verstehst du, was ich damit sagen will?'

'Ich bin ein Kind, aber ich bin nicht dumm!'

'Nun, weißt du etwas Tiefes über die Welt?'

'Vieles.'

'Verrätst du mir etwas davon?'

'Ich weiß etwas über Geheimnisse. Es ist wichtig, welche zu haben.'

'So? Was noch?'

'Ich weiß etwas über die Menschen. Im Mai sitzen sie alle draußen und tun so, als wären sie glücklich. Stimmt's?'

'Das stimmt. Was noch?'

'Über Menschen weiß ich schon einiges. Die meisten von ihnen leben nicht richtig, sie glauben es nur. Und viele sind nur für mich da, die gibt es gar nicht wirklich.'

'Du weißt eine Menge für dein Alter.'

'Ich weiß noch mehr. Ich weiß, dass ein Erwachsener, der einem Kind solche Fragen stellt, ziemlich seltsam ist.'

Picco lachte. ${ }^{631}$

Glavinic operiert mit kurzen, einfach strukturierten Sätzen. Diese setzen sich aus einfachem, wie im Fall von 'Schickschnack' alltagssprachlichem und kindgerechtem Vokabular zusammen. Er führt kindertypische Szenarien wie den Schulbesuch oder das Zähneputzen an, zu denen jeder Leser leicht Zugang finden sollte. Ein komplexes Fachwissen ist nicht von Nöten, um den Dialog nachzuvollziehen, lediglich alltägliche Erfahrungen. Hinsichtlich der Funktion ergibt sich der deutlichste Unterschied zu Mann daraus, dass die intellektuell überlegene Figur keinerlei didaktische Absichten verfolgt, während Settembrini und Naphta stets versuchen, Castorp, und damit auch den Leser, für sich zu gewinnen. Glavinics Ziel ist eher eine Bestandsaufnahme denn eine Belehrung des Jüngeren. Dies spiegelt sich auf der Ebene der Kommunikation zwischen Autor und Leser. Dadurch, dass die philosophischen Ansichten von einem Kind verlangt werden, können sie, durch die realistische Herangehensweise legitimiert, als vage Andeutungen verbleiben, was Glavinic durch die ironisch selbstreflexive Aussage, dass es ohnehin komisch sei, mit Kindern über so etwas zu sprechen, abrundet. Für den jungen Leser liefert der Bezug zu seiner Alltagswelt ein hohes Identifikationspotenzial. Für den erfahreneren und reflektierten Leser wird einerseits genug Ansatz geliefert, um 
beispielsweise darüber nachzudenken, was es heißt, glücklich zu sein. Andererseits wird ihm kein philosophischer Kontext vorgegeben, sondern Freiraum beim Füllen der bewusst gesetzten Textlücken gelassen. ${ }^{632}$ Dadurch erhält der Leser bei der Lektüre eine aktive Rolle, die auf seine Emanzipation in Bezug auf die Texthoheit schließen lässt, die der Autor durch die bewusst lesbare Gestaltung und durch die selbstreflexiv hinterfragte, realistische Gestaltung des Kindseins legitimiert.

Auch Mann greift im Zauberberg auf Selbstreflexivität zurück, um textuelle Gestaltungsweisen zulässig zu machen, die seines Erachtens nicht angemessen sind. Nach der Schilderung von Castorps Schmerz über den Abschied Clawdia Chauchats greift der Erzähler kommentierend ein: 'Man wird urteilen, der Erzähler trage dick und romantisch auf [...]. Und dennoch [...] halten [wir] uns genau an unseres schlichten Helden persönliches Gemüt, dessen Kenntnis uns auf eine Weise, die sich freilich der Untersuchung entzieht, gegeben ist [...].' ${ }^{633}$ Mann macht eine Überlegenheit des Erzählers gegenüber den Lesern geltend. Diese Souveränität im Umgang mit den Figuren und dem Text, die sich jedweder Prüfung entzieht und Einwänden der Kritik vorbeugt, zementiert die Position des allwissenden Erzählers und legitimiert die daraus resultierende Gestaltungsweise.

Gekoppelt an das Liebessujet evoziert Manns Beschreibung der emotionalen Gestaltungsweise den Begriff des 'Kitsch', den Hans-Edwin Friedrich im Reallexikon folgendermaßen definiert: 'Negativ wertender Begriff, meist als Oppositionsbegriff zu “Kunst" verwendet'. ${ }^{634}$ Friedrich führt aus, dass Kitsch in seiner Begriffsgeschichte ob einer vermeintlich unkontrollierten Sentimentalität als 'Ausgangspunkt für die Abwertung der Massenliteratur' verwendet worden war. ${ }^{635}$ Kitsch bewegt sich demnach in dem Spannungsfeld zwischen Kunst und massentauglicher Literatur - die selbstreflexive Aussage des Erzählers im Zauberberg positioniert Kitsch ebenfalls in Opposition zur Kunst. Kitsch kann also als der Breitenwirkung förderliches Mittel situiert werden.

Dass sich Glavinics Verlag von den Elementen des Liebesromans hohe Verkaufszahlen versprochen hatte, zeigt die Marketingstrategie. So wurde der Roman

\footnotetext{
632 Auch Wiele, der sich in der $F A Z$ äußerst negativ zum Roman äußert, bezeichnet eine ähnliche Stelle gegen Ende des Romans als Jonas und Marie wieder zusammentreffen als 'hohe Kunst der Aussparung' und 'beste Stelle', was dieser Strategie Qualität zuerkennt; vgl. Wiele 2013 und Glavinic 2013 a, S. 522.

${ }^{633}$ Mann 2013, S. 863.

634 Friedrich 2000, S. 263.

635 Vgl. ebd., S. 264.
} 
im Klappentext als 'Thomas Glavinics großes Buch der Liebe' und auf Glavinics Website als 'ein Buch der Liebe' beworben. ${ }^{636}$ In der ZDF-Sendung Das blaue Sofa entgegnet Glavinic auf den Kitschvorwurf der Kritik: 'Es gibt [...] Leute, die mir [...] vorgeworfen haben, [...] dass es ein bisschen kitschig sei. Gut, es ist ein Liebesroman. Liebe ist ja, also Liebe, die funktioniert, ist ja sowieso immer kitschig. ${ }^{637}$ Glavinic weist den Kitsch-Verdacht nicht von sich, setzt sich aber gegen den Banalitätvorwurf zur Wehr und etabliert Kitsch als notwendiges Gestaltungsmittel. Anders als bei Mann resultiert diese Überzeugung nicht aus einer vom Autor beanspruchten Texthoheit, sondern aus dem Wunsch der mimetischen Nachahmung von Liebesbeziehungen. Glavinic beansprucht Kitsch als legitimes Gestaltungsmittel, das mit einem Kunstanspruch zu vereinen ist. Dies kann als Indiz dafür gewertet werden, dass qualitativ ambitionierte Autoren im Zuge des Lesbarkeitsaktivismus zusehends auf traditionell verpönte Mittel zurückzugreifen. ${ }^{638}$

Hinsichtlich des Kitsches lohnt ein letzter Vergleich mit Manns Zauberberg. Während Jonas wieder mit Marie zusammenkommt, lässt Clawdia Castorp im Sanatorium zurück. Bezeichnend ist die Szene, in der Jonas bei stürmischem Wetter vom Everest absteigt. Er will aufgeben, ehe Marie ihn überzeugt abzusteigen. ${ }^{639}$ Die Konstellation entspricht der Castorps als dieser im Schneesturm gefangen unter Todesvisionen leidet. Castorp gibt wegen Clawdia nicht auf und kommt zu der Erkenntnis: 'Die Liebe steht dem Tod entgegen, nur sie, nicht die Vernunft, ist stärker als er.' ${ }^{640}$ In beiden Fällen rettet die Liebe den Protagonisten. Wie auch bei den philosophischen Fragestellungen führt Mann seinen Erkenntnisprozess mit einer komplexen Syntax aus, während Glavinic auf kurze, prägnante Dialoge setzt. Beide Stellen erfüllen jedoch die gleiche Funktion.

Allgemein spart Mann während der an Goethes Walpurgisnacht erinnernden Karnevalsepisode nicht mit kitschigen Elementen: 'wie ein sonderbar tiefer Traum, denn man muß sehr tief schlafen, um so zu träumen... Ich wollte sagen: Das ist mir ein längstvertrauter Traum, ein schon immer geträumter Traum, ein langer, ewiger Traum,

\footnotetext{
636 Vgl. Glavinic 2013 b.

637 Herles 2013 b.

638 In der Kritik kam es ob des Kitsches zu einem Streit zwischen den Feuilletons der FAZ, des Spiegel und der SZ, während die $F A Z$ den Roman deswegen abkanzelt, revidiert dies der Spiegel und lobt wegen der 'Grenzüberschreitung' den Kitsch wie die $S Z$ als spannendes neues Gestaltungsmittel, das mit Qualität versehen wird: 'Ein kitschiges Buch. Aber ein großes Buch.' Vgl. Wiele 2013, Becker T. 2013 und Magenau 2013.

639 Vgl. Glavinic 2013 a, S. $515 f$.

640 Mann 2013, S. 679.
} 
ja, so neben dir zu sitzen wie jetzt - das ist selige Ewigkeit. ${ }^{941}$ Mann leitet die Stelle durch ständige Wiederholungen und Variationen aus dem Wortfeld des Träumens in ein pathetisches Ende mit einem Ausblick auf Ewigkeit und Seligkeit. Wenn die Moderne und Mann als deren Vertreter als Höhepunkt ambitionierter Qualitätsliteratur gesehen wurde, so muss gefragt werden, ob in deren Rezeption die Gestaltungsmittel der ULiteratur nicht bewusst ausgeblendet wurden. Die Debatte um die Lesbarkeit hätte dann das Repertoire der qualitativ hochwertigen Literatur nicht etwa erweitert, sondern ein Umdenken eingeleitet: Autoren können Mittel wie Kitsch nicht nur verwenden, sondern deren literarisches Potenzial ausschöpfen, ohne sich für deren Verwendung vor der Kritik rechtfertigen zu müssen.

Krausser, Kehlmann und Glavinic operierten zunächst in einem Kontext, der noch eindeutig von den zentralen, aus der deutschen Literaturgeschichte tradierten Streitpunkte des Literaturstreits geprägt war. Sowohl in ihren eigenen primären und sekundären Werken als auch in deren Rezeption zeichnet sich zu Beginn des 21. Jahrhunderts eine klare Tendenz ab, dass der Kampf für Lesbarkeit tiefgreifende Veränderungen initiierte, die den deutschen Literaturbetrieb nachhaltig beeinflussen sollten. Dabei muss hervorgehoben werden, dass es Autoren waren, die durch strategische Zusammenarbeit sowohl die theoretische Fundierung als auch die praktische Umsetzung vorantrieben, und die anderen Vertreter der Literaturproduktion und -rezeption für Lesbarkeit gewannen. Der Umschwung hin zur Lesbarkeit führte zudem auch im Ausland zum Erfolg bei Publikum und Kritik, wodurch die internationale Reputation der deutschen Literatur reevaluiert wurde. Kraussers, Kehlmanns und Glavinics Romane verdeutlichen Strategien zur Wahrung des Qualitätsund des Lesbarkeitsanspruchs, wobei die Autoren auf ähnliche Mittel rekurrieren und dadurch eine Basis an Techniken liefern, die zur Erschließung einer Lesbarkeitstradition im frühen 21. Jahrhundert beitragen kann.

641 Ebd., S. 464 (französisches Original) bzw. 991 (Übersetzung). Im Original erscheint die Stelle auf Französisch, wodurch der Autor seine polyglotte Bildung ausstellt und dadurch den nicht-frankophonen Teil der Leserschaft ausschließt und womöglich den Kitsch legitimiert. 


\section{Kapitel 5: Alte und neue Formen der Lesbarkeit. Felicitas Hoppe und Ulrike Draesner}

Felicitas Hoppes und Ulrike Draesners Poetologien liefern kritische Gegenstimmen zum Lesbarkeitsparadigma. Oppositionelle Positionen in die vorliegende Arbeit einzubeziehen, liegt in Thomas Kuhns Paradigmentheorie begründet. Er schreibt, dass ein Paradigma nie ohne 'counterinstances' bestehen könne. ${ }^{642}$ Dies entspricht dem der Lesbarkeitsdebatte inhärenten Wettstreitcharakter: Ein Paradigma wird durch Gegenstimmen nicht etwa geschmälert, sondern bestätigt. Dies könne dann wiederum zu einer erneuten Krise mit Paradigmenwechsel führen. ${ }^{643}$ Daher wäre es auch im Hinblick auf den dynamischen Charakter des Lesbarkeitsbegriffs nicht förderlich, eine klare Grenze zwischen lesbarer und unlesbarer Literatur und den jeweiligen Autoren zu ziehen. Vielmehr soll es unternommen werden, am Beispiel von Hoppe und Draesner die Vielfalt und das kreative Potenzial des Paradigmas anzudeuten. Dies soll wie bei Helmut Krausser, Daniel Kehlmann und Thomas Glavinic durch eine kombinierte Analyse von Theorie und Praxis geschehen. Die theoretischen Schriften sollen dezidiert auf Stellen untersucht werden, die Aspekte des Lesbarkeitsbegriffs diskutieren. Hoppes Kinderbuch Iwein Löwenritter (2008) und Draesners Roman Sieben Sprünge vom Rand der Welt (2014) dienen dazu aufzuzeigen, dass die Autorinnen trotz ihrer kritischen Haltung auf Lesbarkeit rekurrieren. Da Lesbarkeit wegen des Zielpublikums ein Genrespezifikum der Kinderliteratur darstellt, soll zudem nachgewiesen werden, dass Hoppe gewisse Lesbarkeitsstrategien aus dem Löwenritter zudem auch in ihrem Roman Hoppe (2012) anwendet, um ein repräsentatives Bild ihres Gesamtwerks zu erhalten und ein den Romanbeispielen aus dem vorhergehenden Kapitel entsprechendes Werk zu behandeln.

\section{Felicitas Hoppe}

In Hoppes Sieben Schätze betitelten Augsburger Poetikvorlesungen (2008/2009) fungiert Lesbarkeit als bedeutsame Kategorie. Hoppe echauffiert sich, dass im 20. Jahrhundert 'so viele große Künstler so viele unlesbare Werke geschrieben haben', die

\footnotetext{
642 Vgl. Kuhn 1996, S. 79.
}

643 Vgl. ebd. 
kaum gelesen wurden und dennoch einflussreich und berühmt sind. ${ }^{644}$ Hoppe positioniert Lesbarkeit als Mittel zur Breitenwirkung im Sinne des Gelesenwerdens. Zudem kann die Passage als Plädoyer dafür verstanden werden, Lesbarkeit als Qualitätsmerkmal großer Kunst zu etablieren. Dennoch werden ihre weiteren poetologischen Ausführungen auf eine kritische Auseinandersetzung mit den Lesbarkeitstendenzen im frühen 21. Jahrhundert verweisen.

Zunächst macht Hoppe zwei Trends in der deutschen Gegenwartsliteratur aus, die für einen nachhaltigen Erfolg der Lesbarkeit im Hinblick auf Breitenwirkung sprechen:

erstens die Großveranstaltung, das Schimpfwort dafür lautet Event, sodass Einzellesungen zunehmend von Festivals abgelöst werden, also Literatur am Block statt Literatur als Fortsetzung von Geschichte und Geschichten. Zweitens die Biografisierung, womit ich nicht die inflationäre Überschüttung des Marktes mit Biografien aller Art meine, sondern den Trend, Werke im Doppelpack mit dem Leben ihres Autors nicht nur zu verknüpfen, sondern auch zu vermarkten.

Beiden Trends entspricht eine sich deutlich verändernde Preislandschaft, in der das, was ich vertikale Preise nenne, der Büchnerpreis ist das prominenteste Beispiel, durch einen Trend zum horizontalen Preis ersetzt wird, wobei ich an die Preise der Buchmesse denke, die in erster Linie hohe Auflagen erzielen sollen. ${ }^{645}$

Beide angeführten Trends deuten auf eine zunehmende Repräsentation von Autoren und deren Werken in der Öffentlichkeit, womit Hoppe die Umsetzung eines zentralen Anliegens der 'Neuen Deutschen Lesbarkeit' und auch der Autoren Krausser, Kehlmann und Glavinic attestiert. Was Hoppe an diesen Trends kritisch betrachtet, ist deren Instrumentalisierung für Marketingzwecke, d.h. Breitenwirkung im Sinne hoher Verkaufszahlen. ${ }^{646}$ Dies drückt sie durch die Höhenmetaphorik bei der Bewertung von Literaturpreisen aus. Die auf den Büchnerpreis angewandte Vertikalität verweist auf Höhen und Tiefen in der Qualität. Horizontalität impliziert bereits in ihrer Metaphorik Breite, die Hoppe unmittelbar mit finanziellem Erfolg in Verbindung setzt. Daran zeigt sich der Einfluss Adornos und Horkheimers und deren Stigma gegenüber kommerziell erfolgreicher Literatur.

\footnotetext{
644 Hoppe 2009 b, S. $216 f$.

645 Ebd. S. 55.

${ }^{646}$ In diesem Kontext müsste auch die Frage nach Poetikvorlesungen gestellt werden, zumal vor allem die Frankfurter Vorlesungen für den Autor nicht nur Prestige, sondern auch eine umfassende Medienberichterstattung nach sich ziehen. Außerdem scheint die eigene Person in fiktionaler Bearbeitung vermehrt den Weg ins eigene Werk zu finden. Sei es durch einen Stellvertreter wie in Daniel Kehlmanns Ruhm, eine unbenannte Autorfigur, die mit dem Bild der eigenen öffentlichen Persona spielt, wie in Helmut Kraussers Eros oder durch die explizite namentliche Einschreibung der Autoren wie in Kraussers UC (2003), Thomas Glavinics Das bin doch ich oder Hoppes Hoppe.
} 
In der Vorlesung 'Stroh zu Gold' geht sie speziell auf finanziellen Erfolg ein. Grundsätzlich betont Hoppe die Komplexität des Marktes, die Autoren und ihre Werke maßgeblich beeinflusst:

\begin{abstract}
Unermüdlich und mit den Jahren in wachsender Fülle und mit steigendem finanziellem und personellem Aufwand schickt [der Markt] den faktischen Lieferungen seiner Produkte in Verlagsvorschauen große Verheißungen und Versprechungen voraus, streut Gerüchte und Bilder (dem Genre des Autorenfotos gebührte eine eigene Vorlesung), schürt Erwartungen und Hoffnungen, rollt Klappentexte wie rote Teppiche aus, stellt Pokale in alle Fensterbänke, lobt Preise, Stipendien, Poetologievorlesungen und Gastprofessuren für wissenschaftlich nur bedingt zurechnungsfähige Schriftsteller aus. Kurz, er versucht, eine Aura zu schaffen, in der Werke erst gedeihen und dann vertrieben werden sollen. Dabei macht er sich eine Fülle von Mitteln zunutze, von denen nicht wenige zweifelhaft sind. 647
\end{abstract}

Hoppe setzt die Beobachtung fort, dass die Literatur zusehends in den öffentlichen Raum drängt, wozu auch die zunehmende Präsenz der Autoren beiträgt. Hier stellt sie dies als dezidiert geplante Strategie der Verlage heraus, um Bücher zu vermarkten. Auffällig ist die Wahl der Bezeichnung 'Produkt' für literarische Werke, was den industriellen Charakter des Verlagswesens zugunsten des künstlerischen in den Vordergrund stellt. Hoppe entwirft eine Vorstellung des literarischen Produktionsprozesses, der einerseits durch die Förderung von Autoren durch Preise, Stipendien oder Vorlesungen Züge eines Mäzenatentums trägt, durch das die Autoren mit ausreichenden finanziellen Mitteln versorgt werden, um ohne Existenzängste schreiben zu können. Andererseits ist jedoch auch diesem System der Marketingaspekt eingeschrieben, der bei der Veröffentlichung eines Buches zur vollen Entfaltung kommt. Hoppe selbst erkennt das Schielen auf Verkaufszahlen als unbestreitbare Realität an, nimmt jedoch zugleich eine kritische Distanz hinsichtlich dieser Entwicklung ein.

An anderer Stelle fügt Hoppe ihrem Bild vom System der Literaturvermarktung weitere Akteure hinzu. Die deutsche Literaturlandschaft, so Hoppe, sei einmalig:

Nicht nur, was ihren Produktionseifer betrifft, sondern auch ihren Ehrgeiz in Bezug auf Förderungen, auf Preise, Stipendien und geförderte Übersetzungen, von ihren Kommentarapparaten ganz $\mathrm{zu}$ schweigen, dem nach wie vor einzigartigen deutschsprachigen Feuilleton, der Wissenschaft, dem Netz und natürlich jeder Menge literarischer Zeitschriften. ${ }^{648}$ 
$\mathrm{Zu}$ den bereits angeführten Institutionen kommen hier sowohl Übersetzungen, die auf die Globalität des Literaturmarkts verweisen, als auch der sekundäre Sektor hinzu. Mit dem Internet bringt Hoppe ein neues Medium ins Spiel, das jedoch nicht wie andere neue Medien in den 1990er Jahren in Konkurrenz zur Literatur erscheint, sondern als weiteres Mittel neben den traditionellen publizistischen und wissenschaftlichen Veröffentlichungen dazu dient, Breitenwirkung herbeizuführen.

Auf dieser Basis stellt Hoppe die Frage nach der Position des Autors im Literaturbetrieb. Sie spricht zunächst von einem 'Spagat zwischen Kunst und Markt [...], von dem Abgrund, der sich auftut zwischen dem Werk und seiner Verwandlung in Ware, im günstigsten Fall in natürliches Gold. ${ }^{649}$ Hoppe eröffnet eine Dichotomie zwischen künstlerischem Anspruch und finanziellem Erfolg. Anhand des Spagat-Bildes macht sie deutlich, dass Autoren zwischen den Polen balancieren, um die von beiden Seiten gestellten Anforderungen zu erfüllen. In der Folge dekonstruiert sie die Metapher umgehend:

[Sie verabschiedet den] Mythos des unkorrumpierbaren Künstlers [...] der in kompromissloser Unabhängigkeit sein Werk schafft, um es danach Zähne knirschend zu Markte zu tragen und dort selbstherrlich und selbstmörderisch zugleich zur Diskussion zu stellen und damit der Öffentlichkeit verfügbar zu machen. (Martin Walser und Günter Grass sind schöne Beispiel für diese Art spätromantischen Gestus.) Aber ist ein Schriftsteller ohne Öffentlichkeit, sprich ohne den Markt, überhaupt ein Schriftsteller? (Walser und Grass wissen bestens, dass es nicht so ist, schicken sie doch gern gelegentlich einem Bucherfolg einen entsprechend produktiven Skandal voraus. $)^{650}$

Hoppe wendet sich gegen die idealistische Vorstellung des 'freien poetischen Kunstwerks'. ${ }^{651}$ Im Gegensatz zu Hegel setzt sie eine stetige Abhängigkeit des Künstlers von nicht-literarischen Faktoren für jedwedes Schaffen als grundlegend voraus. Zudem geht sie konkret auf die Einbettung der Literaturproduktion in den Markt ein. Zum einen zeichnet sich in der Erwähnung der Autoren Walser und Grass ein Generationenkonflikt ab, den z.B. auch der Guardian in seinem Kehlmann-Porträt am Beispiel ebenjener Autoren geltend macht. Zum anderen konstatiert Hoppe, dass der

\footnotetext{
649 Ebd., S. 40.

650 Ebd., S. 41.

651 Dass Hoppe auch von der Freiheit des Kunstwerks spricht, zeigt sich in folgender Passage: '[Es] stellt sich immer wieder die Frage nach dem, was es wahrscheinlich nie gegeben hat: die Frage nach der Autonomie der Kunst, dieser diffuse und immer etwas verstockte Versuch einer Selbstvergewisserung, Ausdruck einer Sehnsucht nach freien und unberührten geistigen Räumen, nach Authentizität (die mit Kunst sehr wenig zu tun hat), die zutiefst romantische Annahme also, es habe tatsächlich einmal so etwas wie künstlerische Freiheit gegeben.' (Ebd., S. 49).
} 
Prozess, ein Buch der Öffentlichkeit über den Markt zugänglich zu machen, konstitutiv für den Status als Schriftsteller ist. Dies führt insofern zu einer Aufwertung der Leserposition, als Autoren in einem direkten Dependenzverhältnis zu ihren Lesern stehen, wobei das Finden von Lesern einen Autor erst zum Autor macht.

Aus dieser Konstellation zwischen Autor, Markt und Leser zieht Hoppe zwei Schlüsse. Zum einen führt die Zurückweisung der Idee des 'freien poetischen Kunstwerks' zu einer Akzeptanz des Geldverdienens, was sie am Beispiel des Minnesängers Walther von der Vogelweide festmacht. Diesen setzt sie als reisenden Dichter zu den Lesereisen der Autoren der Gegenwart in Bezug. Sie schreibt: 'Die Kunst ist nicht frei, aber sie versorgt ihn am 12. November 1203 immerhin mit Geld für einen Mantel. ${ }^{652}$ Das Beispiel zeigt, dass Hoppe unter dem Geldverdienen wie Krausser den Erwerb lebensnotwendiger Produkte versteht. Einen direkten Bezug zwischen finanzieller Absicherung und der Qualität des Werkes stellt sie anders als z.B. Glavinic nicht her.

Zum anderen kommentiert Hoppe anhand ihres Bilds vom Literaturmarkt die Autor-Leser-Konstellation. Sie hält zunächst eine Deutungshoheit der Verlagsvertreter hinsichtlich des Publikumsgeschmacks für nicht tragbar:

Nur was wissen [die Verlagsvertreter] wirklich von [des Lesers] Wünschen? Ich habe den Leser als solchen nie persönlich getroffen. Trotzdem, oder eben deshalb, glaube ich an eine ernsthafte Beziehung zwischen Autor und Leser. In meiner naiven Vorstellung ist dies eine Beziehung von gleich $\mathrm{zu}$ gleich, [...] ohne Hierarchien und auf produktive Weise respektlos, dabei diskret und auf befreiende Weise distanziert, weil sie etwas mit echten und einfachen Bedürfnissen zu tun hat, zum Beispiel mit dem Wunsch danach, ernst genommen zu werden und dabei auf Vorschriften und Einreden von außen zu verzichten.

Eine Beziehung, die keine Märtyrer oder Opfer verlangt. Der Autor säße nicht länger unter dem Damoklesschwert permanenter Identifikationsangebote, sein Leser wäre vom Identifikationszwang befreit. ${ }^{653}$

Hoppe verweist auf die Problematik der Personifikation eines heterogenen und diversen Publikums. Wie das Programm der 'Neuen Deutschen Lesbarkeit' fordert sie daher, den Leser ernst zu nehmen, verfolgt dabei allerdings eine andere Strategie: Während erstere propagierte, aktiv auf den Leser zuzugehen, wählt Hoppe den Weg der vollkommenen Indifferenz. Ordnet man dies in Hoppes vorherige Aussage ein, dass sich Autorschaft erst über das Gelesen-Werden konstituiert, dann sollen die Leser von Autoren nicht zur 
Lektüre verführt, sondern ihnen freie Hand bei der Lektürewahl überlassen werden. Die Autoren hingegen gewinnen dadurch eine größere Freiheit von textexternen Faktoren. Durch beider Freiheit ergibt sich dann das von Hoppe ersehnte egalitäre Verhältnis.

Hoppe erkennt in der Literaturlandschaft zwei Vorgänge, die eine solche Gleichstellung verhindern. Als erste Ursache benennt Hoppe, dass der Leser durch die neuen technologischen Möglichkeiten der Meinungsverbreitung über das Internet zusehends in der Hierarchie nach oben wandert:

\begin{abstract}
Der Grund dafür, dass die Rechnung zwischen Autor und Leser so selten aufgeht, liegt aber mittlerweile längst nicht mehr in der aufsässigen Belehrung des Lesers durch den Autor, sondern, umgekehrt, in der möglichen Belehrung des Autors durch den Leser, der im Internet unter dem Siegel demokratisch verbürgter Meinungsfreiheit nach Feierabend im Meinungsblog sein lässiges Urteil fällt. Das Ergebnis ist so einfach wie zwingend: Wir suchen gar keine Partnerschaften, sondern die schlichte Umkehrung der Hierarchien, die die vertrauten Herrschaftsverhältnisse umdreht, ohne sie anzutasten. ${ }^{654}$
\end{abstract}

Hoppe lehnt die Abneigung der Leserschaft gegenüber einer didaktischen Belehrung als Grund für das Ungleichgewicht ab, da sie deren Zurückweisung im Literaturstreit in der Praxis umgesetzt sieht. ${ }^{655}$ Vielmehr macht sie eine Umkehrung des Kräfteverhältnisses aus, die den Leser als Richter über den Autor inthronisiert. Hoppe erkennt an, dass die Hierarchien unangetastet bleiben, und etabliert zugleich deren Abschaffung als einzigen Weg zu einer Gleichstellung von Autoren und Lesern.

Den zweiten Grund für die Spannungen auf dem Literaturmarkt sieht Hoppe darin, dass die gesamte Literaturproduktion zusehends auf Kompromissen beruht. Alle Vertreter des Marktes, die sich entweder unter Schreibenden oder Lesenden einordnen ließen, wollen Hoppe zufolge dasselbe:

das herausragende, originelle Individuum und sein Werk auf der einen Seite und den überragenden und überraschenden (finanziellen) Erfolg auf der anderen, der heute mehr denn je auf Konsens beruht, auf Entgegenkommen von allen Seiten.

Der romantische Geniekult hat dabei einem pragmatischen, aber deshalb nicht weniger Kräfte raubenden Geschäftssinn Platz gemacht. Autoren sind Lieferanten, Verleger Kreativindustrielle geworden. [...]

Übersättigte und so gut wie tabufreie Gesellschaften sind [...] vor allem harmoniesüchtig. Nur wie stellt man Harmonie in Pluralität her? Indem man sich an die Grundregeln hält: erstens überraschen, zweitens bestätigen. [...] Autonomie

\footnotetext{
654 Ebd., S. 58f.

655 An anderer Stelle führt Hoppe die Taktik des 'Süßens' als einzige Strategie zur Verarbeitung von politischem Gedankengut an: 'Wo derzeit [...] Politik in der Literatur auftaucht, ist sie mehr theatralische Pose als ernsthaftes Anliegen. Wenn sie verdaut werden soll, muss sie verpackt werden, nach wie vor am besten in Liebesgeschichten' (Ebd. S. 128).
} 
auf der einen, Dienstbarkeit auf der anderen Seite. Der Leser will überrascht werden, gerne auch hier und da bewegt, in Maßen erschüttert, aber immer auf bekannte und verdauliche Weise und in geordneten Bahnen. ${ }^{656}$

Hoppe stellt Originalität und Qualität eines Werks in Gegensatz zu finanziellem Erfolg. An finanziell erfolgreichen Werken kritisiert sie, dass jene aufgrund von Kompromissen zustande kommen. Dies steht zwar im Gegensatz zur Beurteilung eines Mittelwegs durch das Programm der 'Neuen Deutschen Lesbarkeit' sowie Krausser, Kehlmann und Glavinic, bekräftigt jedoch deren Annahme, dass eine solche Literatur in der deutschen Literatur der frühen 2000er Jahre für Verkaufserfolg garantiere. Adornos und Horkheimers idealistische Ablehnung eines industriellen Charakters prägen Hoppes weitere Ausführungen: Sie erkennt, dass sich die Autoren den Gegebenheiten einer 'Kulturindustrie', deren Regeln so maßgeblichen Einfluss auf die Literaturproduktion nehmen, unterordnen und den Schreibenden somit eine erhabene Genie-Position entzogen würde. Dies belegt sie durch die Annahme, dass die Autoren beim Schreiben immer auch die Bedürfnisse des Lesers beachten, was ein Grundgedanke der 'Neuen Deutschen Lesbarkeit' ist, und damit ihre künstlerische Unabhängigkeit unterlaufen. Als von den Lesern bevorzugte Wirkungsabsicht beobachtet Hoppe unterschiedliche Abstufungen des movere, die 'verdaulich' gestaltet sein sollen. Somit rekurriert Hoppe auf eine für den Lesbarkeitsdiskurs traditionelle Metapher aus dem Bereich der Nahrungsaufnahme, die Lesbarkeit als leicht verständliche Textgestaltung definiert. Diese Entwicklungen lehnt sie deswegen ab, da sie der Umsetzung des von ihr bevorzugten indifferenten doch respektvoll egalitären Verhältnisses zwischen Autor und Leser im Weg stünden.

In ihren Vorlesungen bespricht sie detailliert weitere zentrale Aspekte des Lesbarkeitsbegriffs: eine realistische Gestaltungsweise, die Dichotomie zwischen Trivial- und Hochliteratur, das Erzählen sowie die rhetorischen Wirkungsabsichten, deren Analyse in ebenjener Reihenfolge abgehandelt werden soll. Hinsichtlich des Realismus sieht Hoppe zwei mögliche literarische Verfahren:

[Ich ziehe] die gesamte Literatur auf zwei einfache Leinen: Auf der einen hängt, was versucht, der Wirklichkeit mit literarischen Mitteln auf die Schliche zu kommen, folglich Wirklichkeit mit literarischen Mitteln simuliert, während auf der anderen hängt, was diesen Prozess umkehrt: Hier wird mit den Mitteln der 
Wirklichkeit Literatur simuliert. Während also im ersten Fall die höchste Kunst darin besteht, eine Geschichte echt erscheinen zu lassen, wie im wirklichen Leben, besteht im zweiten Fall die Kunst darin, die Wirklichkeit so erscheinen zu lassen, als sei sie nichts als reine Erfindung, womöglich ein Märchen. [...]

Selbstverständlich ist die Frage danach, an welcher Leine das eigene Schreiben hängt, keine Frage nach der Qualität der jeweiligen Literatur [...].657

Der erste Pol ist die klassisch aristotelische Mimesis. Der zweite zielt darauf eine Wirklichkeit zu erschaffen, die nicht auf der Realität gründet. Da Hoppe als Beispiel das Genre des Märchens anführt, kann angenommen werden, dass das Ziel des zweiten Pols hauptsächlich im Verarbeiten fantastischer Elemente besteht, was mit Aristoteles' Mimesisbegriff $\mathrm{zu}$ vereinbaren ist, solange dadurch eine kohärente fiktionale Welt entworfen wird, in der diese Elemente realistisch sind. Ebenso positioniert sich Hoppe durch diesen Realismusbegriff in der Nähe Kehlmanns, dessen Vorliebe für fantastische Literatur jedoch nicht dem Märchen entstammt, sondern dem realismo mágico.

Hoppe geht dann hinsichtlich des Realismus auf die Frage nach der Qualität ein. Für die Rezeption des Realismus in Deutschland folgert sie am Beispiel ihres Reiseromans Pigafetta (1999):

\begin{abstract}
Dagegen steht der reine Erfinder auf den ersten Blick wesentlich besser da, denn er hat sich schließlich nur vorgestellt, was der Gegenstand seiner Reise ist. Der Rest wird gefüllt durch die Einbildungskraft, ein besonderes Vorrecht der Deutschen, die die Idee in der Regel höher hängen als das, was wir erfahrene Wirklichkeit nennen. ${ }^{658}$
\end{abstract}

Hoppe deutet auf eine Privilegierung des romantischen Geniegedankens gegenüber dem Realismus hin. Dass sie Romantik und Realismus direkt kontrastiert, ist folgerichtig; so kreiert beispielsweise auch Fontane sein Realismusprogramm aus Ablehnung gegen eine realismusferne Ästhetik der Romantik und macht zugleich den höchsten Qualitätsanspruch für den Realismus geltend. Da Hoppe selbst tatsächlich reist, teilt sie Fontanes Auffassung und attackiert die qualitativen Vorbehalte gegenüber einer realistischen Gestaltung.

Zur Qualitätseinordnung greift Hoppe auf Höhenmetaphorik zurück und geht explizit auf das Verhältnis zwischen hoher Literatur und Leserzahlen ein. Sie folgert, dass 'Kunst, die Höhe hat, so gut wie nicht gelesen wird' ${ }^{659}$ An dieser Aussage sind für 
Lesbarkeit zwei Komponenten relevant. Erstens erscheint Kunst nicht an Höhe gebunden. D.h. das Prädikat Kunst kann auch mittleren oder niedrigen Werken zuerkannt werden, was dem künstlerischem Selbstverständnis der 'Neuen Deutschen Lesbarkeit' entspricht. Zweitens stimmt Hoppe mit deren Literaturprogramm dahingehend überein, dass ambitionierte hohe Literatur wenig gelesen wird. Die Literatur, die ein breites Publikum anspricht, muss dementsprechend höhenmetaphorisch ausgedrückt darunter angesiedelt sein.

In der Folge setzt sich Hoppe kritisch mit der höhenmetaphorischen Hierarchie auseinander:

Der Begriff der Hohen Literatur leitet fehl, denn auch das Höchste schlägt sich physikalisch nieder in der Luft, die wir atmen, so wie auch Niedriges, ob wir nun wollen oder nicht, früher oder später unvermeidlich nach oben steigt. Alles, die Feuchtgebiete unserer Literatur inklusive, ist mit Tradition gesättigt, mit Mythen und Märchen, mit Geschichten, Erinnerungen und Faustregeln, mit lauter kleinen Teilen ungelesener Bücher, die sich als Teil unserer Kultur ständig in Gegenwart verwandeln. ${ }^{660}$

Hoppe zeigt auf, dass die Pole der hohen und der niedrigen Literatur künstliche Konstruktionen sind. Natürlicherweise existieren demnach nur Mischformen, die sich aus Elementen beider Pole speisen. Diesen Mix beansprucht z.B. Krausser als zentral für seine Autorpoetik. Zudem wird aus dem Zitat ersichtlich, dass die Zugehörigkeit der Elemente zu den Polen stetem Wandel unterworfen ist und zu verschiedenen Zeiten unterschiedlich rezipiert werden kann. Dies gilt in Anbetracht seiner Tradition auch für den Lesbarkeitsbegriff. Mit der Anspielung auf Charlotte Roches Bestseller Feuchtgebiete (2008) deutet Hoppe darauf hin, dass auch in den vermeintlichen Niederungen in der Kultur verankerte traditionelle Elemente verarbeitet werden, auf denen die Werke der hohen Literatur gründen, was nicht nur die Niederungen als ebenbürtigen Teil der Kulturlandschaft etabliert, sondern erneut die höhenmetaphorische Unterteilung anprangert. Allerdings findet sich Hoppe entgegen ihren persönlichen Neigungen mit den Gegebenheiten des deutschen Literaturbetriebs ab: 'Aber jenseits der Freiheiten in diesem Spiel bleibt, und das ist verständlich, seit jeher das Bedürfnis nach Ordnung und damit, jenseits aller demokratischen Verlautbarungen, ein Bedürfnis nach Rängen und Hierarchien.' ${ }^{661}$ Hoppe greift Hierarchien zwar als unzureichend an 
und fordert deren Auflösung, erkennt dennoch deren Funktion, Literatur kategorisierbar zu machen, an. Dabei darf nicht vergessen werden, dass das Pochen der Vertreter der Lesbarkeit auf eine mittlere Literatur ebenfalls die Beibehaltung von höhenmetaphorischen Hierarchien forciert.

Ebenso skeptisch beäugt Hoppe bei ihrer Analyse des Verhältnisses zwischen Autoren und der Germanistik eine ästhetische Entwicklung der Gegenwartsliteratur hin zum Erzählen, das einen immanenten Aspekt des Lesbarkeitsbegriffs darstellt. Hoppe erläutert im Kontext von Podiumsdiskussionen zwischen Schriftstellern und Akademikern:

Und doch legen [...] die meisten Podiumsgespräche von einer zunehmend friedlichen und produktiven Koexistenz Zeugnis ab. Autoren und Wissenschaftler sitzen Seite an Seite unter universitären Glasglocken, befragen sich nach den Quellen ihrer Geschichten, bescheinigen sich gegenseitig, dass wir ja alle irgendwie bloß Geschichten erzählen und nichts als artige Arbeiter in der großen Narrationsfabrik sind.662

Hoppe macht geltend, dass sowohl durch Autoren als auch durch die Wissenschaft das Erzählen als vorherrschendes Paradigma angesehen wird. Dass sie diesen Zustand nicht unbedingt gutheißt, zeigt sich an der Verwendung des Begriffs 'Narrationsfabrik', der Adornos und Horkheimers Terminus der 'Kulturindustrie' resoniert. Erzählende Literatur wird somit zur schablonenhaften Massenware. 663

Im Hinblick auf das Erzählen führt Hoppe eine Bemängelung ihres eigenen Werks an. Sie erklärt, dass ihr vom Beginn ihrer Karriere an von Seiten der Kritik der Vorwurf gemacht worden sei, 'nichts zu erzählen' zu haben. ${ }^{664}$ Hierbei geht es weniger um eine reservierte Haltung gegenüber dem Erzählen als um eine Inhaltslosigkeit der Geschichten. Hoppe geben die Beanstandungen der Rezensionen Anlass zu einer Reflexion über ihren geringen finanziellen Erfolg. Sie kommt zu dem Schluss, dass 'es beim Schreiben nicht um die Wahrheit geht, sondern darum Wirkungen zu erzielen und Rührung zu erzeugen. Wäre ich bei diesem Programm geblieben, wäre ich heute unter

\footnotetext{
662 Ebd., S. 214.

663 Die Annahme, dass auch in akademischen Arbeiten zunehmend erzählt wird, müsste in einer separaten Studie auf Validität und eventuelle Zusammenhänge mit dem Erzähldesiderat in der Literatur geprüft werden. Die Narratologie geht jedoch davon aus, dass auch wissenschaftliche Publikationen vermehrt narrative Züge tragen. Vgl. Herman 2007 b, S. 4-6. Hoppe selbst schreibt: 'Ob die in diesem Austausch zunehmend zerfließenden Grenzen der Literatur mehr nützen als schaden, sei dahingestellt, wie auch die Antwort auf die Frage, ob der in der Wissenschaft in Mode gekommene Hang zum Erzählen in Bezug auf ihren Gegenstand Früchte trägt.' (Hoppe 2009 b, S. 214).

664 Ebd., S. 113.
} 
Umständen erfolgreich.' ${ }^{665}$ Die Betonung der Wirkung auf den Leser entspricht der Breitenwirkungsstrategie des Programms der 'Neuen Deutschen Lesbarkeit'. Dabei setzt Hoppe jedoch nicht primär auf das delectare, sondern auf das movere, worin sie Krausser ähnelt. Ungewöhnlich ist an Hoppes Ausführungen die Opposition von Wahrheit und Wirkung. Traditionell ist Wahrheit ein Begriff, der im Gegensatz zu einer reinen Abbildung der Wirklichkeit auf Qualität verweist. Die Wirkungsabsichten stehen in der aristotelischen Tradition eigentlich in einem symbiotischen Verhältnis zur Wahrheit und werden durch jene nicht eingeschränkt.

Obwohl Hoppe selbst dem movere zugetan ist, erkennt sie Unterhaltung als dominante Funktion in der Gegenwart an: 'In der Gegenwartsliteratur [nimmt man], was gefällt, und lässt weg, was beschwert.' ${ }^{666}$ Auch hier zieht Hoppe eine ungewöhnliche Gegenüberstellung heran. Sie stellt die Wirkungsabsicht des Erfreuens nicht den anderen Funktionen gegenüber, sondern der Komplexität eines Textes. Dies verweist darauf, dass dem delectare automatisch eine einfache Textgestalt zugeschrieben wird. Später führt sie den Terminus einer 'intellektuelle[n] Erbauungsliteratur' ein, von der es gerade eine neue Welle gebe, die sie ablehne.667 Die Verbindung der religiösen Erbauungsmetapher und des Intellekts steht für eine Unterhaltungsliteratur, die sich durch das intellektuelle Sujet eine höhere Qualität verspricht. Eine solche zur Mitte tendierende Strategie weist Hoppe von sich.

Abschließend kann konstatiert werden, dass Hoppe eine ambige und oftmals oszillierende Position zu Lesbarkeit einnimmt, deren paradigmatische Stellung sie jedoch bestätigt: 'Wir [die Leser] haben uns längst an Gläser gewöhnt, an geschliffene Ränder, an leichtes und unbeschwertes Trinken, Schluckbeschwerden mögen wir nicht.' ${ }^{668}$ Hoppe bemüht erneut eine Metapher aus dem Bereich der Nahrungs- bzw. Flüssigkeitsaufnahme. Das Bild des Glasschleifens steht für eine künstlerische Bearbeitung, die dem Konsumenten das Lesen erleichtert und angenehm gestaltet. Zudem erkennt Hoppe, dass im Literaturbetrieb der frühen 2000 Jahre Schwierigkeiten und Hindernisse zugunsten einer leicht rezipierbaren Literatur möglichst vermieden werden. Obwohl sie also das Paradigma der Lesbarkeit anerkennt, nimmt sie für ihre eigene Literatur eine kritische Distanz ein.

\footnotetext{
665 Ebd., S. 114.

666 Ebd., S. 149f.

667 Vgl. ebd., S. 216.

668 Ebd., S. 126.
} 
Die künstlerische Freiheit, auf Lesbarkeit rekurrieren zu können, erlangt Hoppe durch Genrekonventionen. Im Interview mit der Frankfurter Rundschau (2012) führt sie an:

Beim 'Iwein' habe ich gemerkt, dass ich mich freier fühle, weil ich mehr zulassen kann, weil ich etwas schreiben konnte, was man im Erwachsenenbuch als Sentimentalität ansehen würde. Man kann aber an dieser scheinbar simplen Form kläglich scheitern. ${ }^{669}$

Hoppe erkennt wie Kästner qualitative Vorbehalte gegenüber der Kinderliteratur. Diese revidiert sie dadurch, dass sie die Anforderungen herausstellt, die das Genre an die Autoren stellt. Dadurch wertet sie zugleich eine einfache Textgestalt auf. Diese macht sich Hoppe für ihr eigenes Werk produktiv zu Nutze. Dass sie auch mit ihrer Kinderliteratur einen hohen Qualitätsanspruch vertritt, zeigen ihre Poetikvorlesungen. Sie etabliert das Kinderbuch sowie das Märchen als hochwertige literarische Formen. Carlo Collodis Pinocchio (1881), das sie als Kinderbuch und Märchen klassifiziert, vergleicht sie mit dem Fragment Der Verschollene (1927) des kanonisierten Autors der Hochliteratur Franz Kafka, wobei sie beide Werke als ebenbürtig ansieht. ${ }^{670}$ Zum Märchen schreibt Hoppe: 'Ich neige [...] zu der leichtfertigen Behauptung, das Märchen sei die höchste, weil einfachste Form realistischer Literatur'. ${ }^{671}$ Leichtigkeit im Sinne einer leichten Zugänglichkeit avanciert zur wichtigsten Qualität, die das Märchen gegenüber anderen realistischen Formen exponiert. Hoppe positioniert beide Gattungen im Kontext der hochliterarischen Tradition, was besonders für Iwein Löwenritter von Bedeutung ist, indem sie sich Hartmanns prestigeträchtiges Original zur Vorlage wählt. Der Qualitätsanspruch, den Hoppe mit einfachen Formen verfolgt, soll abschließend daran festgemacht werden, dass sie auch in ihrem Erwachsenenbuch Hoppe Lesbarkeitsstrategien anwendet.

Dass Lesbarkeit ein inhärentes Gattungsmerkmal der Kinderliteratur ist, macht Kümmerling-Meibauer geltend: Die Kinderliteratur zeichne sich durch spezifische Merkmale wie 'Einfachheit, Redundanz und die Nachahmung mündlichen Erzählens' aus. ${ }^{672}$ Redundanz kann als Mittel gewertet werden, den Rezipienten durch eine Vielzahl

\footnotetext{
669 Geißler 2012.

670 Vgl. ebd., S. 86-88. In diesem Kontext könnte eine Analyse von Kraussers Kinder- und Jugendbuch Die wilden Hunde von Pompeii (2004) und der in den Tagebüchern vermerkten poetologischen Motivationen erfolgen.

671 Ebd., S. 19.

672 Kümmerling-Meibauer 2000, S. 254.
} 
an Informationen die Orientierung im Text zu erleichtern. Der mimetische Rückgriff auf gesprochene Sprache kann ebenfalls als Mittel angesehen werden, den in ihrer Lesekompetenz noch nicht ausgereiften Kindern durch die vertrautere mündliche Kommunikationsweise die Lektüre zu erleichtern. Im Hinblick auf die Ablehnung einer didaktischen Funktion von Literatur im Literaturstreit wird auch die in der Kinderliteratur oftmals angestrebte pädagogische Wirkung zu beobachten sein. ${ }^{673}$

$\mathrm{Zu}$ Beginn von Iwein Löwenritter evoziert der Erzähler unmittelbar sowohl einen mündlichen als auch einen didaktischen Kontext: 'Also vergesst die Schule, und stellt euch stattdessen einen Wald vor, einen Wald wie im Märchen. Finster und voller Geräusche und voller unsichtbarer Tiere. Das ist der Wald von vor tausend Jahren, der Immerwald.' ${ }^{674}$ Zum einen zeigt die Stelle, dass die Erzählung an Kinder adressiert ist, zum anderen eröffnet sie zwei Referenzsysteme, die den Lesern die Orientierung im Text erleichtern. Das erste ist das Genre des Märchens, das Hoppe zusammen mit der Datierung 'von vor tausend Jahren' dazu heranzieht, um einen nicht realistischen Schauplatz zu etablieren. Dessen Beschreibung ist so neutral gehalten, dass sie der Imagination der Leser großen Spielraum lässt, ihn mit ihrer eigenen Fantasie auszugestalten und sie dadurch zu einer aktiven Lektüre einlädt. Der als Erzähler fungierende anthropomorphisierte Löwe stellt zudem ein dem Genre der Fabel entlehntes fantastisches Element dar. ${ }^{675}$ Das zweite Referenzsystem stammt aus dem Alltag der Kinder: die Schule. Zunächst werden sie durch direkte Ansprache zur aktiven Teilnahme am Textgeschehen eingeladen. ${ }^{676}$ Dann wird dem fantastischen Märchensetting ein reales Szenario entgegengesetzt, mit dem sich die Leser unmittelbar identifizieren können, wodurch ihnen das Geschehen leicht zugänglich gemacht wird.

Die Veranschaulichung des Erzählten durch Verweise auf die Schule ziehen sich durch die gesamte Erzählung. In Bezug auf die Gattungsmerkmale der Kinderliteratur kann von einer bewusst eingesetzten Redundanz gesprochen werden, die dezidiert auf die Vereinfachung der Lektüre zielt. Neben dem Referenzsystem Schule soll im Verlauf der weiteren Analyse das Augenmerk auf folgende stets wiederkehrende Aspekte gelenkt werden, die Lesbarkeit fördern: den Einsatz von Farben zur Wiedererkennung

\footnotetext{
673 Vgl. ebd., S. 254 und $256 f$.

674 Hoppe 2009 a, S. 10.

675 Vgl. ebd., S. 250.

676 Die Partizipationsfunktion der fingierten Mündlichkeit, die dem Erfahrungsbereich der Kinder entlehnt ist und dadurch deren Textverständnis fördert, wird auch von der Narratologie behandelt. Vgl. Norrick 2007, vor allem S. 134f.
} 
und Charakterisierung der Figuren, die konsequente Verwendung gleicher Ausdrücke zur Beschreibung derselben Sachverhalte und klarifizierende Passagen, die zur Leitung der Leser durch den Text dienen.

Hoppe erzählt in zwei Teilen, 30 kurzen Kapiteln auf 250 Seiten und in vier Illustrationen, im Duktus des mittelalterlichen Prätextes chronologisch Iweins Âventiuren folgend. Dabei versieht sie sowohl die beiden Teile als auch die Kapitel mit thematischen Überschriften, die einen zentralen Aspekt des folgenden Abschnitts aufgreifend einen Orientierungsrahmen setzen. Die Teile beispielsweise verweisen mit ihren Titeln 'Iwein' und 'Der Löwenritter' auf die vom Protagonisten vollzogene Entwicklung und ergeben zusammengenommen den Titel des Romans. ${ }^{677}$ Die ersten vier Kapitel legen den jeweiligen Schauplatz fest und manifestieren einen Schauplatzwechsel im Vergleich zum vorherigen Kapitel (Immerwald, Artushof, auf der Reise, Das Land nebenan). Später dienen die Überschriften ebenso zur Benennung der zentralen Figuren, zur Hervorhebung gewisser bedeutender Gegenstände und sich zu vollziehender Handlungen, oder etablieren wie im Falle des 23. Kapitels, 'Der rote Sonntag nach Ostern', einen Zeitsprung in der Handlung. ${ }^{678}$ Von dieser im aristotelischen Sinne stringenten Erzählweise gibt es zwei Abweichungen: Erstens die durch die Prolepse hervorgehobene, in der Chronologie erst später anzusiedelnden Szene des Drachenkampfs zu Beginn des Romans und zweitens die Nebenhandlung des Streits zwischen den beiden Schwestern.

Die Darstellung des Drachenkampfs nimmt das Ende des ersten Kapitels ein. Kapitel 2, 'Der Hof von vor tausend Jahren', beginnt dann mit der Vorgeschichte, die erzählt, wie Iwein vom Artushof auszog. Die ersten Sätze lauten: ‘Aber wenn ihr wissen wollt, wie die Geschichte jetzt weiter geht, dann müsst ihr erst wissen, was vorher geschah. Denn die Geschichte fängt nicht im Immerwald an, sondern in einem anderen Land. Das ist das Land von vor tausend Jahren. ${ }^{679}$ Wie dessen Überschrift zeigt die Einführung in das Kapitel den Ortswechsel unmittelbar an. Zudem ist wie in der Überschrift der Ort an eine zeitliche Dimension gebunden, die Hoppe mit der Formulierung 'von vor tausend Jahren' mit der von der Beschreibung des 'Immerwalds' bekannten zum Ausdruck bringt. Die direkte Ansprache der Leser betont die Absicht,

\footnotetext{
677 Vgl. Hoppe 2009 a, S. 7 und S. 129.

678 Ebd., S. 98.

679 Ebd., S. 15.
} 
dem Leser einen Sachverhalt verständlich machen zu wollen. In Kombination mit den klar markierten Orts- und Zeitwechseln entstehen so möglichst wenige Rezeptionsschwierigkeiten.

Dieselbe Strategie verfolgt Hoppe zu Beginn des zweiten Teils, wo die Passage in ihrer chronologisch adäquaten Position angeführt wird. Zunächst verortet der Erzähler die Handlung durch eine direkte Ansprache der Leser im bereits bekannten 'Immerwald': 'Erinnert ihr euch an den Immerwald? An diesen finsteren Wald von vor tausend Jahren, ohne Wege und Schilder, in dem sich selbst Ritter und Könige verirren? Wo die Bäume so hoch in den Himmel wachsen, dass man keinen Himmel mehr sieht?' ${ }^{680}$ Auffällig ist die wiederkehrende Formulierung 'von vor tausend Jahren'. Jedoch entsprechen auch die aufgeworfenen Fragen exakt der zu Beginn gegebenen Beschreibung des Walds. ${ }^{681}$ Die Wiederholung der Muster vereinfacht dem Leser den vom Erzähler in Gang gesetzten Erinnerungsprozess. Nach einer kurzen Beschreibung von Iweins Reise durch den Wald werden die Leser dann in ihrem Wissen um das Geschehen bekräftigt: 'Ihr kennt diese Lichtung, und ihr kennt auch die Tiere, die in wildem Streit miteinander liegen.' ${ }^{6} 82$ Der Leser wird durch das Evozieren einer Komplizenschaft an Vorheriges erinnert. Somit gesteht der Erzähler dem Leser die Kompetenz zu, der Handlung folgen zu können. Der ständige Dialog mit dem Leser über die Ansprache durch den Erzähler erweckt die gattungstypische mündliche Kommunikationsweise, die durch ihre Nähe zum realen Umfeld der Kinder den Bezug zum Text erleichtert.

Der Übergang der Haupthandlung zur Geschichte der beiden Schwestern erfolgt von Kapitel $8 \mathrm{zu}$ Kapitel 9 des zweiten Teils über eine thematische Anknüpfung an Iweins Abenteuer. Nachdem Iwein und der Löwe im Kampf verwundet wurden, setzt sich der Erzähler mit dem Tod auseinander:

Und während [Iwein und der Löwe] zwischen dem Land Nebenan und dem Land Tausendburgen im Tiefschlaf lagen, geschahen da draußen viele Dinge, wie das im Leben der Menschen so üblich ist.

Denn die Menschen kommen selten zur Ruhe. Wenn sie leben, kämpfen sie, und wenn die Kämpfe zu Ende sind, werden sie alt und müssen sterben. ${ }^{683}$

\footnotetext{
680 Ebd., S. 131.

681 Vgl. ebd., S. 10.

682 Ebd., S. 132.

683 Ebd., S. 163.
} 
Das folgende Kapitel beginnt mit einer Passage über den im Sterben liegenden Vater der beiden Schwestern: 'So war es auch im Land Tausendburgen. Dort lag krank der Burgherr der tausendsten Burg und wartete auf seinen letzten Gast. Er war nicht nur krank, sondern auch sehr alt und wusste, dass die Zeit zum Sterben gekommen war.' 684 Die Einbettung der Nebenhandlung erfolgt sowohl thematisch als auch über die Lokalität. In beiden Textstellen fungiert das Land Tausendburgen, das dem Leser aus Iweins vorherigen Abenteuern bekannt ist, als lokaler Bezugspunkt, der den Übergang einleitet. Zudem thematisieren das Ende des achten und der Beginn des neunten Kapitels beide den Tod, wobei Hoppe in beiden Abschnitten mit demselben Vokabular das Sterben mit dem Alter verknüpft. Zudem eröffnet sie am Ende von Kapitel 8 die Dichotomie zwischen Bewegung und Ruhe, wobei sie erstere mit dem Leben und letztere mit dem Tod verbindet. Das erste Hauptverb von Kapitel 9, 'lag', bedeutet durch den Rückgriff auf die Dichotomie Stillstand und Tod, was Hoppe durch Krankheit und die Betonung des Alters durch das Adverb 'sehr' noch intensiviert. Zuletzt findet sich in diesem Übergang am Beispiel des Tiefschlafs der Protagonisten und des Fortschreitens der Dinge an anderen Orten eine selbstreflexive Angabe, die den Einschub der Nebenhandlung einführt. Es handelt sich um eine im Text motivierte und folgerichtige Pause der Haupthandlung, die einen Spielraum zur Abweichung auf die Nebenhandlung eröffnet. Als Legitimation wird die Nachahmung des realen Lebens angeführt, was die Stelle nahe an die Alltagswelt der Kinder rückt. Der Übergang ist demnach anschaulich vorbereitet, sodass er für die Leserschaft leicht nachzuvollziehen ist.

Zur Analyse der Lesbarkeit muss auch der Endpunkt der Nebenhandlung beachtet werden. Aristoteles macht geltend, dass Nebenhandlungen dann legitim sind, wenn sie die Haupthandlung voran treiben. Die Nebenhandlung der Geschichte der beiden Schwestern führt direkt in die finale Konfrontation zwischen Iwein und Gawein. Die jüngere Schwester begibt sich auf die Suche nach Iwein, während der sie die vorherigen Stationen von Iweins Âventiure zurückverfolgt bis sie ihn schließlich findet. Die Nebenhandlung mündet exakt an der Stelle in die Haupthandlung, an der sie von ihr abgewichen war: 'Sie erkannte ihn gleich, was nicht weiter schwer war. Iwein lag immer noch schlafend neben dem Waldweg unter den Bäumen und Büschen. ${ }^{685}$ Die jüngere Schwester erfährt im Verlauf ihrer Reise niemals, dass Iwein im Wald schläft. 
D.h. an diesem Merkmal kann sie ihn nicht erkennen. Dessen Hervorhebung zielt direkt auf den Leser, da die Situation exakt dem Szenario entspricht, an dem die Haupthandlung vorübergehend aussetzt. Die prominente Herausstellung zu Beginn und deren Aufgreifen am Ende der Nebenhandlung erleichtern dem Rezipienten die Wiederanknüpfung an Iweins Âventiure, die sich dann vollends durch seinen Aufbruch zum Artushof, also durch die Aufnahme von Bewegung, manifestiert, was wiederum auf die Dichotomie zwischen Bewegung-Leben und Stillstand-Tod verweist. Die Nebenhandlung ist mit leicht nachvollziehbaren Anknüpfungspunkten in die Erzählung eingebaut, treibt die Haupthandlung voran und entspricht somit dem in der vorliegenden Arbeit etablierten Lesbarkeitsbegriff.

Das Duell zwischen Iwein und Gawein ist speziell aufgrund des Einsatzes von Farben zu betrachten, welcher als Mittel fungiert, um die Lesbarkeit des Textes zu garantieren. Iwein und Gawein sind die einzigen Figuren des Buches, deren Farbgebung sich ändert. Im dritten Kapitel wird die Freundschaft der beiden im Rahmen eines Festes am Artushof eingeführt: 'Auf der anderen Seite des Königs saß Ritter Gawein. Diesen Namen müsst ihr euch merken, das ist der beste Freund von Iwein. Oder auch nicht. Das wird sich noch zeigen. ${ }^{686}$ Hoppe gibt dem Leser eine explizite Lektüreanleitung, die sein Augenmerk auf die Entwicklung des Verhältnisses der Freunde lenkt und Spannung auf die Auflösung des Sachverhalts erzeugt. In Bezug auf die Farben wird seit dem Kapitel davor der Artushof stets mit der Farbe Blau assoziiert. ${ }^{687}$ Blau steht zum einen für die Zugehörigkeit zum Artushof und zum anderen in der Form des blauen Bandes, für ritterlichen Ruhm. Als Iwein und Gawein zahlreiche blaue Bänder gewinnen, werden die beiden mit Spitznamen versehen: 'Deshalb nannte man sie auch die blauen Ritter oder die blauen Zwillinge.' ${ }^{888}$ Nachdem somit die Farbgebung zunächst die Einheit der beiden Ritter etabliert, zeigt sie dann den Umschwung des Freundschaftsverhältnisses an:

Kaum hatte Lunete den Platz verlassen, wurde es totenstill, alle Zelte verloren plötzlich die Farbe.

\footnotetext{
686 Ebd., S. 19.

687 Z.B. 'Stellt euch jetzt also den Königshof vor. Dort ist der Himmel immer blau. Morgens hellblau und abends dunkelblau', 'Und wenn am Ende der Beste gewinnt, bekommt er zur Belohnung ein blaues Band und bindet es seinem Pferd in die Mähne. Das ist das berühmte blaue Band', 'So war es auch an einem Sonntag vor Ostern, der schön war und blau' (Ebd., S. 15, 16 f und 19).

688 Ebd., S. 95.
} 
Und alle, die Lunetes Rede gehört hatten, begriffen, dass Iwein kein blauer Ritter mehr war und auch kein blauer Zwilling. Er war jetzt vollkommen allein.

Das begriff auch Gawein. ${ }^{689}$

Der vollkommene Farbverlust symbolisiert den Bruch zwischen den beiden Rittern. Iwein verliert die mit der Farbe Blau verknüpfte Ehre, die Verbindung zum Artushof sowie die Freundschaft zu Gawein.

Nach diesem Zustand der tabula rasa durchläuft Iwein diverse Stadien der Farbzugehörigkeit. Während seiner Verzweiflung nach Lunetes Kundgebung flüchtet er, wobei 'sein Körper allmählich schwarz' wurde. ${ }^{690}$ Heilung findet er durch die weiße Herrin der weißen Burg mit den weißen Händen und dem weißen Pferd, die Iwein in ein weißes Gewand kleidet und auf ein Pferd setzt, das 'weiß wie Sahne' war. ${ }^{691}$ Hoppe spielt mit der gängigen Dichotomie zwischen hell und dunkel bzw. gut und böse, die sie im weiteren Verlauf des Romans beibehält, um die Figuren durch die Farbzuteilung einem der beiden Pole zuzuordnen. ${ }^{692}$

Dass Iwein und Gawein nichts von ihrer Gegnerschaft wissen, resultiert ebenfalls aus der Farbzuordnung. Sie haben die blauen Zwillinge zurückgelassen und kleiden sich in Weiß bzw. Grün: 'Weil er unbekannt bleiben wollte, trug Gawein nicht wie sonst seine blaue Rüstung, sondern eine grüne. Denn Grün ist die Farbe der Hoffnung.' ${ }^{693}$ Hoppe rekurriert auf die im Deutschen gängige Redensart von der Bedeutung der Farbe Grün. Durch die unterschiedliche Farbgebung zeigt sie zum einen die Gegnerschaft der beiden Ritter, andererseits wird die Bedeutung das Kampfes hervorgehoben, da der in Blau ruhmreiche Gawein sich eines Sieges aus eigener Kraft nicht sicher sein kann. Dadurch greift Hoppe zudem auf die zu Beginn des Romans verwiesene Infragestellung des Freundschaftsverhältnisses zurück, die sich im Zweikampf manifestiert. Als der Kampf in einem Unentschieden endet und die beiden Freunde sich erkennen, wird umgehend von der Rückkehr der blauen Zwillinge gesprochen. ${ }^{694}$ Dies löst die Frage nach dem Verhältnis der beiden auf, da beide den mit der Farbe Blau verbundenen

\footnotetext{
689 Ebd., S. 106.

690 Ebd., S. 108.

691 Vgl. ebd., S. 117-121, direkt zitierter Ausdruck auf S. 124.

692 Besonders wichtig ist diese Dichotomie, um den Lesern den Zugang zur komplex ambigen Janus-Figur des 'doppelten Ritters' zu erleichtern, der sowohl ein weißes als auch ein schwarzes Gesicht hat und dadurch seine Gegner verwirrt. Vgl. ebd., S. $198 f$.

693 Ebd., S. 211.

694 Ebd., S. 222.
} 
Ruhm wiedererlangt haben und zumindest vorübergehend wieder in den Artushof eingegliedert sind. Der bedeutungsträchtige Farbwechsel dient zu einer symbolischen Veranschaulichung der dynamischen Veränderung der Freundschaft sowie von Iweins Erfolg in seinem Streben nach ritterlicher Ehre.

Zuletzt steht die Bedeutung der Farbe Rot im Kontrast zum Blau des Artushofs für das Land Nebenan: 'Hinter der Falltür sah [Iwein] die Burg. Die war prächtig und groß, hoch und stark und überall mit roten Fahnen geschmückt.' ${ }^{95}$ Die Burg im Land Nebenan und der Artushof werden durch die Verbindungen $\mathrm{zu}$ der jeweiligen Grundfarbe eindeutig voneinander abgegrenzt, und im weiteren Verlauf werden die wichtigsten Protagonisten des jeweiligen Ortes mit der entsprechenden Farbe ausgestattet. Im Land Nebenan tritt Laudine, Iweins Frau, ab dem Besuch von Artus in einem markanten roten Mantel auf. ${ }^{696}$ Als Iwein $\mathrm{zu}$ seiner Âventiure aufbricht, überreicht ihm Laudine einen mit einem roten Stein besetzten Ring:

Ich liebe dich mehr als mein Leben. Noch nie habe ich einen Mann so geliebt wie dich. Deshalb gebe ich dir diesen Ring, niemandem sonst würde ich ihn geben. [...] Aber vergiss eins nicht: Heut ist der rote Sonntag nach Ostern, und bist du in einem Jahr nicht zurück, genau auf den Tag, dann ist das für unser Land ein Unglück. Für dich und für mich aber wäre es schlimmer als der Tod. ${ }^{697}$

Die Formulierung, jemanden mehr als sein Leben zu lieben, wird im Löwenritter durchgehend verwendet, um die Intensität von Liebesbeziehungen auszudrücken und ist demnach in seiner Repetition an dieser Stelle bekannt. ${ }^{698}$ An zitierter Stelle fungiert sie zudem gemeinsam mit der Evokation des Todes als Rahmung. Dies ist zum einen der traditionelle, der griechischen Mythologie entlehnte, Topos von Eros und Thanatos. Zum anderen funktioniert die Rahmung auch ohne den mythologischen Hintergrund als konsequente Weiterführung des Anfangsgedankens von der Liebe, die bedeutender ist als das eigene Leben, sodass deren Verlust im Tod enden würde. Hoppe koppelt die Farbe Rot semantisch an die Liebesthematik, was einem in der Alltagswelt gängigen Schema entspricht. Außerdem fungiert die Farbe als Abgrenzung vom Artushof, denn der Roman führt diesen an einem blauen Sonntag vor Ostern ein. ${ }^{699}$ Sie etabliert

\footnotetext{
695 Ebd., S. 41.

696 'Also zog sich Laudine ihren roten Mantel an und ließ ein rotes Tischtuch auflegen.' (Ebd., S. 82).

697 Ebd., S. 93.

698 Vgl. u.a. ebd., S. 17 und 82.

699 Vgl. ebd., S. 19.
} 
demnach neben den Lokalitätszuordnungen einen Konflikt zwischen Liebe (rot) und Ruhm (blau).

Diese Auseinandersetzung entfaltet Hoppe, als Iwein vom Ablauf der Frist in Kenntnis gesetzt wird. ${ }^{700}$ Bei einem Turnier in Gegenwart Artus' wird zunächst von den blauen Bändern, die Iwein und Gawein ruhmreich gewonnen hatten, gesprochen, ehe die Erinnerung an Iweins Versprechen unmittelbar über die Farbassoziation eintritt:

Der rote Blitz aus dem Stein des Ringes fuhr mitten in seine Vergesslichkeit und brachte die Erinnerung zurück. Der Ring war ja rot wie der rote Sonntag.

Der rote Sonntag nach Ostern!

Da sah Iwein plötzlich Laudine vor sich, wie sie als Herrin vom Land Nebenan in ihrem roten Mantel am Burgtor steht und aus der Ferne zum Abschied winkt. ${ }^{701}$

Dieser Passage liegen alle Aspekte der Rot-Blau-Divergenz zugrunde. Die zeitliche Komponente des roten Sonntags, die örtliche Abgrenzung zwischen dem Artushof und dem Land Nebenan, die mit den Farben verbundenen Figuren sowie der Konflikt zwischen Ruhm und Liebe. Letzteres entwickelt sich dahingehend weiter, dass Lunete in einem 'hellrote[n] Mantel', der sie als Sprachrohr Laudines mit dem Land Nebenan und der Liebesbeziehung verbindet, auftritt und Iwein den Ring mit dem roten Stein abnimmt. ${ }^{702}$ Nach den 'schwarzen' und 'weißen' Perioden, die einen Verlust sowohl von Liebe (rot) als auch Ruhm (blau) anzeigen, geht Iwein am Ende vollends in den roten Bereich über: 'Iwein trug eine rote Rüstung, weil er jetzt wieder Burgherr war. Und was trug er am Finger? Ihr habt es bestimmt nicht vergessen. Er trug den Ring mit dem roten Stein. Den Ring zum roten Sonntag nach Ostern [...].' ${ }^{703}$ Den gesamten Roman hindurch veranschaulicht Hoppe die Entwicklung der Figur Iwein - vom ehrgeizigen Ritter des Artushofs zum Ehemann und Herrn des Landes Nebenan - durch die Farbgebung. Es handelt sich um eine visuelle Komponente, die an das Prinzip der Mise-en-Scène im Film erinnert. Dabei legt Hoppe von Beginn an die verschiedenen Assoziationen leicht nachvollziehbar fest und arbeitet dann das bekannte Muster konsequent und mit denselben Formulierungen sowie Gegenständen und Figuren aus, um die Handlungsentwicklung örtlich, zeitlich und thematisch für den Leser leicht verständlich

\footnotetext{
700 An die Formalitäten des Pakts erinnert der Erzähler zum Ende des vorhergehenden Kapitel 'Blaue Bänder'; das Kapitel selbst trägt den Namen 'Der rote Sonntag nach Ostern', sodass mit Hilfe der Farbunterscheidungen ein leicht nachzuvollziehender Übergang geschaffen wird, vgl. ebd., S. 97f.

701 Ebd., S. 99.

702 Ebd., S. 102-105.

703 Ebd., S. 248.
} 
zu machen. Zusammen mit Beispielen aus der Alltagswelt und der auf Mündlichkeit angelegten Sprache, durch die der Leser Rezeptionsanweisungen erhält und aktiv zur Mitarbeit aufgefordert wird, stellt sie die Lesbarkeit des Romans sicher.

Es bleibt noch die Frage nach einer pädagogischen Funktion zu klären, die in Iwein Löwenritter auch an die Frage nach der literarischen Qualität gebunden ist. Hoppe zieht mit Hartmanns Iwein eines der prestigeträchtigsten Werke des mediävistischen Kanons heran - eine Quelle, die der E-Kategorie zugeordnet werden kann, die mit dem Genre der Kinderliteratur aus der U-Kategorie vermischt wird, wodurch sie ihre Agenda, den Kunstcharakter des Kinderbuchs und Märchens auszustellen, forciert. Während Hoppe aus dem Quellentext das in einzelne Abenteuersituationen gegliederte Erzählmuster und die häufige Verwendung von Superlativen übernimmt, dekonstruiert sie aus heutiger Sicht problematische Vorstellungen von Femininität und Maskulinität. ${ }^{704}$ Im Löwenritter erlauben es die Höflichkeitskonventionen den Frauen nicht, die Ritter im Schach zu besiegen. ${ }^{705}$ Diese Sitte ist ein Hauptgrund dafür, dass sich Iwein vom Hof lossagt und ins Land Nebenan aufbricht. Der Oberhofmeister Keie rät ihm zum Aufbruch: 'Jenseits der Grenze im Land Nebenan leben [...] auch richtige Frauen. [...] Nicht Frauen, die [...] aus Höflichkeit im Schachspiel verlieren.' ${ }^{706}$ Keie erhebt Anspruch auf eine Deutungshoheit in Bezug darauf, was eine Frau ausmacht. Dabei bezieht er sich auf die Unabhängigkeit von höfischen Regeln und ein selbstbewusstes, egalitäres Auftreten beim Schachspiel mit den Männern. Im weiteren Verlauf verkörpert dies Lunete, die am Artushof deswegen von allen außer Iwein schlecht behandelt wurde, weil sie Gawein im Schach besiegt hatte. ${ }^{707}$ In diesem Kontext erscheint Iwein als feministisch inspirierter Charakter, der für die Selbstbestimmtheit von Frauen eintritt. Dass dies Wirkung auf den Artushof hat, zeigen die Feierlichkeiten nach Iweins und Gaweins Zweikampf, während derer die Frauen schließlich die Ritter besiegen. ${ }^{708}$

Iweins Auffassung von Männlichkeit hingegen weicht zunächst nicht von Hartmanns Vorlage ab. Erst im Laufe der Erzählung setzt Hoppe einen Prozess in Gang. Bei Hartmann äußert der Ritter Kalogrenant stellvertretend für die Tafelrunde:

\footnotetext{
${ }^{704} \mathrm{Zu}$ Gender in der deutschen Nachkriegsliteratur vgl. Paul 2009.

705 Vgl. Hoppe 2009 a, S. $15 f$.

706 Ebd., S. 21.

707 Vgl. ebd., S. 42f; bei Hartmann vertritt Lunete zwar emanzipatorische Gedanken, fügt sich aber in die Konventionen. Als sie auf ihre Hinrichtung wartet, äußert sie gegenüber Iwein, selbst für sich kämpfen zu wollen, jedoch sei ihr das aufgrund ihres Geschlechts nicht möglich: 'wan ich bin leider ein wîp / daz ich mich mit kampfe iht wer' (Hartmann 2012, Vers 4072f, S. 238).

708 Vgl. Hoppe 2009 a, S. 227.
} 
ich heizze ein rîter und hân den sin,

daz ich suochende rîte

einen man, der mit mir strîte,

und der gewäfnet sî als ich.

daz prîset in und sleht er mich.

gesige aber ich im an,

sô hât man mich fur einen man [...]. ${ }^{709}$

Bei Hoppe spiegelt sich dieser Ansatz in Iweins Rede:

Ich nenne mich Ritter und habe im Sinn, dahin zu reiten und [...] einen Mann zu suchen, der bewaffnet ist wie ich und mit mir kämpft. Es erhöht seinen Ruhm, wenn er mich schlägt. Aber wenn ich ihn besiege, dann bin ich endlich ein richtiger Mann und habe mehr Ehre als bisher. [...]

Ehre [...] das ist, was ein Mann braucht, um ein Mann zu sein, und ein Ritter, um ein Ritter zu sein. Die Rechnung ist einfach. Je mehr Ehre ein Mann im Leib hat, desto mehr ist dieser Mann ein Mann. ${ }^{710}$

Beide Passagen koppeln Ansehen und Ruhm eines Ritters an den Beweis der Männlichkeit. Männlichkeit wiederum definiert sich über den erfolgreichen Wettstreit mit einem gleichwertigen Gegner. Hoppe betont die Bedeutung des Desiderats, ein richtiger Mann zu sein, noch intensiver und erläutert sie der besseren Verständlichkeit wegen ausgiebig. Zudem bereitet dies die spätere Diskussion der Maskulinität vor, nachdem Lunete Iwein vom Ablaufen der Frist in Kenntnis setzt:

Wären da nicht die anderen gewesen, vor denen Iwein sich schämte, weil er ein Mann war, dann hätte er sich ins Gras geworfen, um mit seiner Sehnsucht allein zu sein.

Aber weil er seine Gefühle nicht zeigen wollte, legte er seinen Kopf in die Mähne des Pferdes und tat so, als würde er ein freies Haar für das tausendste Band suchen. Wenn ihr mich fragt, ich glaube, er weinte. ${ }^{711}$

In dieser Episode wird Iwein durch genderspezifische Erwartungshaltungen eingeschränkt. Gefühle zu zeigen, wird als nicht-männliches Spezifikum angesehen, das zwar mit seinem Charakter, nicht aber mit den an ihn gestellten Erwartungen zu vereinbaren ist. Speziell das Weinen wird mit dem Band, das für den ritterlichen/

\footnotetext{
${ }^{709}$ Hartmann 2012, Vers 530-536, S. 34.

${ }^{710}$ Hoppe 2009 a, S. 29f.

${ }^{711}$ Ebd., S. 99.
} 
männlichen Ruhm steht, kontrastiert. ${ }^{712}$ Das Weinen schließt die Entwicklung Iweins in einem Kontext ab, der ihm nach dem Duell mit Gawein höchsten Ruhm garantiert:

Die Tränen liefen ihnen über die Gesichter und über die Brust, und um sie herum bildete sich mitten im Staub ein See aus Tränen.

Wenn ihr glaubt, dass die beiden sich dafür schämten, weil sie Ritter und Männer waren, dann täuscht ihr euch. Iwein und Gawein schämten sich kein bisschen. Echtes Glück kennt nämlich keine Scham. ${ }^{713}$

Erneut werden Ritterlichkeit und Männlichkeit zunächst in Opposition zum Weinen gesetzt. Es kommt jedoch zu einer Auflösung dieser Spannung, in der das Weinen aus dem Kontext der gängigen Konventionen gelöst und in die exponierte Kategorie des Glücks eingeordnet wird. Dadurch werden Gendernormen gebrochen, was sich bei Hartmann nicht findet. ${ }^{714}$

Am markantesten zeigt sich der emanzipierte Gedanke in der Thematisierung des Herztauschs zwischen Iwein und Laudine. Das weibliche und männliche Herz und deren Tausch werden auch in Hartmanns Roman besprochen:

nû dunchet mîne sinne,
daz mîn her Îwein sî verlorn
sît er sîn herce hât verchorn:
wan daz gap im ellen unde kraft.
waz touc er nû ze rîterschaft?
er muoz verzagen als ein wîp
sît wîbes herze hât sîn lîp
und sî mannes herce hât:
sô uebet sî mannliche tât
und sollte auf Turniere reiten,
und solde wol tuornieren varn
und er dâ heime daz hûs bewarn.
mir ist zwâre starche leit,
daz sich ihr beider gewonheit
mit wehsel sô verchêrt hât.715

Der als 'Ich' sprechende Erzähler Hartmann manifestiert die strikte Unterscheidung zwischen genderspezifischen Attributen und etabliert eine Vermischung jener als schlechtmöglichstes Szenario für beide Geschlechter. Zwar verweist ihn die

\footnotetext{
712 Dass Weinen zunächst mit Weiblichkeit in Bezug gebracht wird, zeigt die Stelle, an der Laudine sich bei Iweins Abreise dazu zwingt, erst zu weinen, als die Männer abgereist waren; vgl. ebd., S. 93.

713 Ebd., S. $219 f$.

714 Dies wäre von Hartmann auch nicht erwartet worden. Hingegen hat die jüngere Forschung den Umstand, dass sich Iwein and Gawein bei Hartmann nach dem Kampf küssen, zum Anlass genommen, die Szene in Bezug auf homoerotische Anklänge unter dem Genderaspekt zu interpretieren; vgl. Kaminski 2001.

715 Hartmann 2012, Vers 2996-3009, S. 176-178.
} 
personifizierte Frau Minne darauf, dass eine solche Mischung von Attributen auch zum Positiven ausschlagen kann, jedoch werden die grundsätzlich mit Männlichkeit und Weiblichkeit assoziierten Aspekte nie kritisch hinterfragt.

Hoppe dagegen kehrt die Attributsverteilung oftmals um. Während der Âventiure reflektiert der Erzähler über Iweins Kampfkraft: 'Wie man sieht, kämpfte er [mit Laudines Herz] besser als je zuvor. Laudines Herz war es, das ihm Kraft und Mut gab und den Wunsch, von Tag zu Tag besser zu werden.' 716 Hoppes Passage unterscheidet sich dahingehend von Hartmann, dass Iweins Turniererfolg gerade dem Herzen Laudines zugeschrieben wird. Anders als in der Vorlage fördern die vermeintlich weiblichen Charaktereigenschaften den Sieg im Wettkampf. Eine weitere Besserung Iweins durch Laudines Herz zieht zunächst negative Folgen für Laudine nach sich. Lunete eröffnet Iwein, dass Laudine in schwerem Kummer lebt und ihr Land vernachlässigt: 'Denn sie trägt jetzt für immer sein Herz in der Brust, das vergesslich ist und nicht weiß, wie man sich kümmert. ${ }^{717}$ Als Iwein später zur Zwangsarbeit gezwungene Frauen sieht, bewegt ihn gerade Laudines Herz zu Mitgefühl und Hilfsbereitschaft.718 Gefühle waren zu Beginn des Romans noch dezidiert als nichtmännlich etabliert worden. Dementsprechend kann die Kritik am Mangel an Mitgefühl und dessen positive besetzte Wiedergutmachung als Aufwertung der Männlichkeit durch der Weiblichkeit entstammende Charakteristika gelten.

Indes bereichern die männlichen Facetten auch Laudine und bewegen sie dazu, ihr Land allein zu regieren. ${ }^{719}$ Am deutlichsten wird dies, als sie selbst ausreitet, um die Gewitterquelle zu verteidigen, was zuvor ihren Ehemännern vorbehalten und ihr ein großes Anliegen bei ihren Hochzeiten war. Lunete äußert gegenüber Laudine: 'Und du bist mutig. Denn du trägst in deiner Brust das Herz eines Mannes und fürchtest weder Wetter noch Wind [...]. ${ }^{720}$ Wie im Original wird Mut als männliche Eigenschaft gesehen. Während bei Hartmann allerdings darüber gespottet wird, dass Laudine auf Turniere reiten soll, veranlasst sie der Mut bei Hoppe dazu, als emanzipierte und souveräne Herrscherin aufzutreten. Die Symbiose aus hybriden femininen und maskulinen Charaktereigenschaften, die Hartmann als Untergang beider Geschlechter

\footnotetext{
716 Hoppe 2009 a, S. 96.

717 Ebd., S. 139.

718 Vgl. ebd., S. 192.

719 Vgl. S. 158.

720 Ebd., S. 237.
} 
ansieht, avanciert bei Hoppe demnach zum Desiderat. Dies zeigt sie in der Szene, in der Iwein und Laudine gegen Ende den Wert des jeweils anderen Herzens gerade ob der jeweils mit dem anderen Geschlecht verbundenen Aspekte preisen und sich dazu entschließen, die Herzen weiterhin vertauscht zu lassen. ${ }^{721}$

Zuteilung und Abgrenzung der Aspekte bleiben wie bei Hartmann unhinterfragt. Allerdings ergibt die Analyse der Intertextualität ein Spannungsfeld zwischen den beiden Versionen. Hoppes Text funktioniert in Bezug auf Lesbarkeit auch ohne das Hintergrundwissen um die Version Hartmanns. Durch das direkte Ansprechen der Leser werden beim Publikum verankerte Genderspezifika, wie z.B., dass Männer nicht weinen dürfen, suggeriert. Der Prozess wiederum kann als didaktisch-pädagogische Funktion des Romans gelten, wodurch Hoppe ein Genremerkmal der Kinderliteratur erfüllt. Dies zeigt, dass Lesbarkeit auch im frühen 21. Jahrhundert mit einer didaktischen Funktion interagieren kann. Zieht man das Wissen um die Textgrundlage und die Abweichungen der Adaption mit ein, so kann Hoppes Spiel mit den Unterschieden als kompetente und kritische Auseinandersetzung mit der literarischen Tradition und qualitativ ansprechende, originelle Bearbeitung gelten. Durch diese Auffächerung kann ein breites Publikum angesprochen werden: Die Zielgruppe der Kinder kann ohne Schwierigkeiten der Handlung folgen, in der Tradition bewanderte Leser werden durch die Entschlüsselung des intertextuellen Spiels unterhalten.

In Hoppe finden sich zwei Anspielungen auf Iwein Löwenritter: Zum einen verwendet Hoppe die Liebte-ihn-mehr-als-mein-Leben-Formel, zum anderen zuweilen den für das mittelalterliche Versepos konstitutiven Superlativ. ${ }^{722} \mathrm{Da}$ sie in ihrem Roman ihre literarischen Einflüsse und eigenen Arbeiten referenziert, kann Iwein Löwenritter als dem Erwachsenenwerk gleichberechtigtes Opus im Hoppe-Corpus gelten. Die Analyse der von ihr angewandten Lesbarkeitsstrategien soll sich zunächst allgemeinen Strategien zuwenden, ehe die Fokussierung der mit dem editorischen Kürzel 'fh' gekennzeichneten Einschübe in eine Diskussion von Lesbarkeit im Kontext des selbstreflexiven Spiels mit der eigenen Persona münden wird.

Hoppe rekurriert auf eine Strategie, die auch Glavinic im Größeren Wunder dazu heranzieht, um möglichst lesbar schreiben zu können. Sie nutzt das Kindsein der 
Protagonistin, die den Namen 'Felicitas Hoppe' trägt, um einer realistischen Prämisse folgend deren einfach gestalteten Erzählungen einschieben zu können. So lautet der Anfang einer versuchten Biographie der Figur 'Felicitas': 'ICH. Meine Familie. Mein Name und meine Wünsche und mein Leben. Felicitas Hoppe. Das bin ich. Im Augenblick, in der Zeit wo ich das schreibe, bin ich zehn Jahre alt. ${ }^{723}$ Während im Löwenritter das Kindesalter des Zielpublikums Lesbarkeit legitimiert, ist es hier die Plausibilität der schriftstellerischen Kompetenz der Figur. Es handelt sich um kurze, simple, oft nicht vollständige Sätze im Nominalstil, die meist parataktisch keine Leseschwierigkeiten ergeben. Zudem evoziert das umgangssprachlich verwendete 'wo' anstatt der korrekten Relativkonstruktion 'in/zu der' einen mündlich kindischen Kontext, der durch die genaue Altersangabe zum Ende abgerundet wird.

Das kinderliterarische Gestaltungsmittel einer evozierten Mündlichkeit schlägt sich auch im regulären Text nieder. Es finden sich zwei verschiedene Anwendungsweisen, die einen impliziten Dialog zwischen Erzähler und Leser kreieren. Der Erzähler äußert bei einer Charakterisierung von 'Hoppes' Vater: 'Erinnern wir uns daran, dass er es war, der ihr die erste Maske baute (“damit sie nicht endet wie Sawchuk”)'. ${ }^{724}$ Es folgt eine Liste von Eigenschaften, die jeweils mit einem erklärenden Satz beendet werden. Der Leser wird durch das inkludierende 'wir' als Komplize des Erzählers eingesetzt, der ihm den Rückblick auf vorher Erzähltes als Lektüremodus vorgibt. ${ }^{725}$ Der genaue Verweis wird dann durch das in Klammern nachgeschobene Zitat erbracht, das es dem Leser erleichtert, die Anspielung nachzuvollziehen. ${ }^{726}$ Die zweite Stelle handelt wiederum vom Vater, 'den wir uns, wollen wir [Hoppes] Erzählungen folgen, als eine Art Rattenund Menschenfänger vorzustellen haben'. ${ }^{727}$ Der Leser erhält konkrete Hilfe zur Einordnung der Vaterfigur. Die Referenz auf den Rattenfänger von Hameln ist ihm bekannt, da die Sage im ersten Kapitel eingeführt wurde, weswegen ihn die Anspielung vor keine Schwierigkeiten stellt. ${ }^{728}$ Der Erzähler nimmt keine didaktisch überlegene Position ein, sondern positioniert sich durch das 'wir' als egalitärer Partner bei der Auseinandersetzung mit den Figuren.

\footnotetext{
723 Ebd., S. 34.

724 Ebd., S. 47, ebenfalls auf S. 182 und 193.

725 Zur Bedeutung der Personalpronomina für die Narratologie siehe Fludernik 2011.

726 Die entsprechende Stelle findet sich in Hoppe 2012., S. 18.

727 Ebd., S. $103 \mathrm{f}$.

728 Zur Einführung der Rattenfängersage vgl. ebd., S. 23f. Klarifizierende Verweise auf frühere Passagen im Roman liefert auch 'fh'; vgl. u.a. ebd., S. 149 und 315.
} 
Komplizierter verfährt Hoppe bei der chronologischen Gestaltung der Handlung. Waren im Löwenritter die Sprünge meist durch eine Kombination aus örtlichen, temporalen und figuralen Markierungen ausgiebig angezeigt worden, sind diese in Hoppe für den Leser oft schwierig nachzuvollziehen. Ein prägnantes Beispiel findet sich im zweiten Kapitel, in dem die Protagonistin von Kanada nach Australien übersiedelt. Ihr Schiff hat den Panamakanal bereits passiert, als sich folgende uneingeführte Stelle findet: 'Bei einem Landgang in Charleston bedrängt sie Kramer so lange mit ihren Bitten [...], dass er sich [...] auf den Weg macht und mit einem [...] Buch [...] zurückkehrt, das sie zwischen Charleston und dem Panamakanal [...] liest'. ${ }^{729}$ Die Analepse ist lediglich durch den Ortswechsel markiert. Dieser stiftet zunächst Verwirrung, da er nicht etabliert wird. Der Reisekontext liefert zwar einen Anhaltspunkt zur Orientierung, jedoch ist diese nur mit einem Vorwissen des Lesers möglich. Erst gegen Ende verbindet Hoppe die Analepse mit dem bekannten Anschlusspunkt Panama, was erneut über eine örtliche Markierung geschieht.

Mit den 'fh' gezeichneten Kommentarkürzeln verfolgt Hoppe zuweilen die auch in der Kinderliteratur prominente Wiederholungsstrategie, um möglichen Unklarheiten vorzubeugen. Paradigmatisch kann die Einarbeitung des Eishockey-Trikots der kanadischen Legende Wayne Gretzky angeführt werden, das die Protagonistin häufig trägt. Die Redundanz funktioniert über drei Informationen: den Spitznamen 'Fly', den 'Felicitas' von der Gretzky-Familie verliehen bekommt, Gretzkys berühmte Nummer 99 und die Signatur 'Wayne for Fly. ${ }^{, 730}$ Die Reihenfolge der Etablierung stellt eine graduelle Einführung von neuen Informationen dar, die zunächst die jeweils für 'Felicitas' und Wayne relevanten Aspekte einführt, ehe beide bekannten Komponenten dann in der Signatur auf dem Trikot mit der Nummer 99 zusammengeführt werden. Obwohl Bedeutung und persönliche Zusammenhänge mit dem Trikot längst etabliert sind, wiederholt Hoppe diese Informationsfülle im weiteren Verlauf des Romans. So heißt es etwa, dass 'Felicitas' immer noch dieses 'Trikot (99/Wayne for Fly/fh)' trägt oder, dass sie nach Gretzkys Hochzeit, die auch den Titel des letzten Kapitels prägt, nun endlich das 'T-Shirt ablegen kann (Wayne for Fly/fh), das [sie] seit Jahren trage [...], obwohl [sie] längst rausgewachsen' ist. ${ }^{731}$ Die Wiederholung erfüllt zwei Funktionen.

\footnotetext{
729 Ebd, S. 107f.

730 'fh' etabliert diese Aspekte ebd., S. 38, 69 und 151. Die Signatur wird zudem auf S. 87 bereits vom Erzähler hinsichtlich einer Fotografie von 'Felicitas' und Wayne eingeführt.

731 Ebd., S. 217 und 317.
} 
Erstens dient sie beinahe pedantisch ironisch dazu, in Bezug auf das Trikot keine Ambiguitäten zuzulassen. Zweitens ist die Beziehung der Protagonistin zu Wayne, den sie heiraten möchte, ein Motor der Handlung. Durch die ständige Erinnerung wird diese konsequent vorangetrieben und das Symbol nachhaltig beim Leser verankert, sodass die emanzipatorische Entwicklung der Protagonistin im zuletzt angeführten Zitat schlüssig abgerundet wird.

Das Kürzel agiert zudem noch mit einer weiteren Strategie, um für die Handlung wichtige Symbole zu verdeutlichen. Ein Beispiel ist der Rucksack, der zu Beginn des Romans als elementarer Bestandteil des Erscheinungsbilds der Protagonistin etabliert wird. ${ }^{732}$ Der Rucksack spielt im Handlungsverlauf u.a. beim Eishockey oder bei der späteren Dirigentenausbildung eine zentrale Rolle. Um darauf vorzubereiten, hebt Hoppe dessen Bedeutung hervor: 'der Rucksack (sic!/fh) auf meinem Rücken'. ${ }^{733}$ Die Aufmerksamkeit des Lesers wird auf den Gegenstand gelenkt, sodass ihm die Lektüreanleitung gegeben wird, auf dessen Wiederkehr zu achten. Sobald der Rucksack erneut eine Rolle spielt, ist der Leser darauf vorbereitet, kennt dessen Relevanz und vermag den Bezug herzustellen. ${ }^{734}$

Das Prinzip der eindeutigen Zuordnung verfolgt Hoppe auch bei literarischen Referenzen, die auf drei verschiedene Weisen erklärt werden. Zunächst beschreibt 'Felicitas' Wayne als 'klein, dünn wie Docht (nur eine von zahlreichen Anspielungen Hoppes auf ihr Lieblingsbuch Carlo Collodis Pinocchio/fh)'. ${ }^{735}$ Die an sich implizite Anspielung auf Pinocchios Reisegefährten wird dezidiert herausgestellt. Damit eröffnet sie auch den Lesern, die die Referenz nicht entschlüsseln können, den Bezugskontext. Denjenigen Lesern, die mit dem Prätext vertraut sind, wird ihr 'esoterisches Vergnügen’ bestätigt. Später im Roman erhält 'Felicitas' ein 'Buch mit dem Titel Schicksal im australischen Busch (Ludwig Leichhardt: Schicksal im australischen Busch. Vorsto $\beta$ in das Herz eines Kontinents/fh)' ${ }^{736}$ Die Verdeutlichung der Referenz hat hier vor allem die Funktion, die scheinbare editorische Seriosität von 'fh' zu unterstreichen. ${ }^{737}$ Sie gibt

\footnotetext{
732 Vgl. ebd., S. 15.

733 Ebd, S. 40.

734 Zudem erreicht dies der Erzähler an anderer Stelle durch die explizite Anweisung an den Leser: 'Wir kommen weiter unten darauf zurück' (Ebd., S. 191).

735 Ebd., S. 18.

736 Ebd., S. 131.

737 Dies gelingt Hoppe auch dadurch, dass ' $f$ ' in ihrer Funktion an Fußnoten und in ihrem Umfang an Lexikoneinträge erinnernde Hintergrundinformationen zu gewissen Sachverhalten liefert. Beispielsweise erläutert 'fh' die Biographie der britischen Königsgemahlin Adelheid von Sachsen-Meiningen; vgl. ebd., S. 108. Außerdem verweist sie in gängiger editorischer Praxis auf Besonderheiten der Typographie; vgl. ebd., S. 164.
} 
Autor und Titel vollständig an und empfiehlt dem Leser dadurch eine weitere Lektüre, um Leichhardts Bericht mit seiner Einbettung in Hoppe abzugleichen. In Australien erhält die Protagonistin schließlich den Spitznamen "CCheshire Cat" (gemeint ist vermutlich die "Grinsekatze" aus L. Carrolls Alice im Wunderland/fh). ${ }^{738}$ Hoppe koppelt die Erklärung an eine Übersetzung. Dadurch mildert sie die durch die Zitation des englischen Originals entstandene Komplexität und ermöglicht durch das deutsche Äquivalent einen weiteren Referenzrahmen. Sie bietet sowohl den mit dem Originaltext als auch den mit der deutschen Übersetzung vertrauten Lesern ein Entschlüsselungsangebot. Zudem wird eine mögliche Sprachbarriere obsolet.

Der Einsatz des Englischen in Hoppe ist aufgrund der Schauplätze Kanada, Australien und USA hinsichtlich einer realistischen Gestaltungsweise konsequent. ' $\mathrm{fh}$ ' geht mit der Verwendung der Fremdsprachen auf drei verschiedene Arten um: An einer Stelle wird sie gar nicht übersetzt. Dort heißt es: 'for additional information or questions, feel free to contact WG Authentic at 1-866-648-4996 or (480) 788-1899 and ask for Drew/fh' ${ }^{739} \mathrm{Da}$ sich die Protagonistin in den USA aufhält, verleiht das Englische der Passage Authentizität. Es handelt sich tatsächlich um eine zusätzliche Information, die die Hauptaussage der Stelle - 'Felicitas' überlegt, wo sich Wayne gerade aufhalten könnte - nicht beeinträchtigt, sodass deren Verständnis nicht unbedingt relevant ist, um der Handlung folgen zu können. Zudem ist der Satz von Zahlen dominiert und in einfacher Sprache gehalten, teilweise in Wörtern, die den deutschen Äquivalenten ähnlich sind, sodass viele deutsche Leser keine Übersetzungsprobleme haben dürften. Dennoch werden all diejenigen Leser, deren Fremdsprachenkompetenz nicht ausreicht, um die Passage zu übersetzen, ausgeschlossen. Eine zweite Methode im Umgang mit der Fremdsprache ist, dass 'fh' eine Übersetzung aus dem Englischen geltend macht, ohne den Originaltext anzugeben. ${ }^{740}$ Zum einen entspricht dies erneut der realistischen Prämisse, da das Original in den kanadischen Jahren von 'Felicitas' entstanden sein soll. 741 Zum anderen zeugt dies von 'fhs' vermeintlich ernsthafter Auffassung ihrer Herausgeberfunktion. Zuletzt übersetzt ' $\mathrm{fh}$ ' ins Deutsche. Sie rekurriert dabei auf Gretzkys tatsächlich erworbenen Titel "'the most gentlemanly

\footnotetext{
738 Ebd., S. 211.

739 Ebd., S. 319.

740 Vgl. ebd.

741 Der Erzähler übersetzt zum selben Zweck auch eine deutsche Passage, die in Kanada auf Englisch gesprochen worden wäre, ins Englische; vgl. ebd., S. 68.
} 
player of all"/“der höflichste Spieler von allen"/fh)'. ${ }^{742}$ Sie nutzt den tatsächlich existierenden Titel des 'most gentlemanly player', um der Aussage Authentizität zu verleihen. Sie kombiniert den Titel mit dem an das mittelalterliche Epos erinnernden Superlativ, um die Eminenz des Sportlers herauszustellen. Sie betont die Polyglottie der Protagonistin und stellt zugleich mit der Übersetzung sicher, dass die Stelle auch einer ausschließlich deutschsprachigen Leserschaft zugänglich bleibt.

Die Kürzel fungieren zudem auch in den ausschließlich deutschen Stellen dezidiert als Mittel zur sprachlichen Verständlichkeit und Klarheit. Zum einen erklärt ' $\mathrm{fh}$ ' schwierig verständliche Fremdwörter: 'Superbia, lat. Hochmut, eine der sieben Hauptsünden/fh).' 743 'fh' situiert die Vokabel sowohl hinsichtlich ihrer etymologischen Herkunft als auch hinsichtlich des Kontexts, in dem sie zu rezipieren ist. Dadurch stattet sie ihre Herausgeberfunktion beibehaltend die Leser mit einer veranschaulichenden Lektürehilfe aus. Dieselbe Funktion erfüllt ebenso die Ausformulierung von Akronymen, die mögliche Zweideutigkeiten verhindert. ${ }^{744}$ Außerdem wirkt ' $\mathrm{fh}$ ' in folgender Passage grammatikalischer Ambiguität entgegen: 'in Paradiese, Übersee (2003) [beschreibt Hoppe] ein Zimmermädchen, das von den Hochzeitsreisenden daran gehindert wird, "ordnungsgemäß”, seiner Arbeit nachzugehen, "weil sie (die Hochzeitsreisenden/fh) befùrchteten, [...] einander wieder abhanden zu kommen"". ${ }^{745}$ Das 'sie' könnte sich auf das Zimmermädchen, die Hochzeitsreisenden und theoretisch, wenn auch wegen der Distanz unwahrscheinlich, auf Hoppe beziehen. Zwar löst das Suffix des anschließenden Werkes die Ambiguität auf, jedoch greift 'fh' präventiv klarifizierend ein und bewahrt den Leser dadurch vor Rezeptionsschwierigeiten.

Es findet sich jedoch eine andere Stelle, an der die vermeintlich gleiche Methodik Ambiguität nach sich zieht. Hoppe fingiert eine Rezension zu ihrem Roman Johanna (2006). Der fiktive Kritiker moniert das von Hoppe im Roman inszenierte Rollenspiel: 'Tatsächlich kommt uns Johannas Ritterrüstung eher wie eine Schuluniform vor, die jene neunmalkluge pubertierende Fanatikerin und selbsternannte Gottestochter (meint er Hoppe, meint er Johanna?/fh) beim besten Willen nicht ablegen kann.' 746 Grammatikalisch kann kein Zweifel bestehen, dass hier Johanna gemeint ist. In diesem

\footnotetext{
742 Ebd., S. 64.

743 Ebd., S. 185.

744 Vgl. u.a. ebd., S. 89 und 95.

745 Ebd., S. 27.

746 Ebd., S. 168.
} 
Sinne ist 'fhs' Eingriff unnötig und erzeugt eine an sich nicht gegebene Diskrepanz. Sie rekurriert jedoch auf das Rollenspiel: In der Aufteilung zwischen Hoppe und ihrer Figur ist der Kern des dynamischen Spiels mit der eigenen Persona in Hoppe angelegt. Diese Diskrepanz forciert 'fh' zudem mit Widersprüchen hinsichtlich der Motivik und der Handlung. Z.B. heißt es: 'Der in Hoppes Werk immer wieder auftauchende Schaukelstuhl ist reine Erfindung, in Hoppes kanadischem Haus gab es keine Gartenmöbel./fh).' ${ }^{747}$ An einer weiteren Stelle interveniert 'fh' hinsichtlich der skifahrerischen Kompetenz eines Charakters: 'Reine Erfindung! Kramer hat nie auf Brettern gestanden./fh). ${ }^{, 78}$ ' $\mathrm{fh}$ ' stellt durch ihre Interventionen den ontologischen Status des Haupttextes in Frage und intensiviert dadurch die Spannung zwischen der realen Autorin, der nach ihr benannten Protagonistin, der Erzählfigur und dem Kürzel 'fh'. Alle stehen in einem dynamischen Austausch, wobei jede Kategorie auf die andere einwirkt und die Grenze zwischen Text und Paratext permeabel wird.

Es stellt sich die Frage, inwiefern die komplexe Aufspaltung Einfluss auf die Lesbarkeit des Romans nimmt. Eine der fingierten Rezensionen im Roman setzt sich selbstreflexiv mit diesem Aspekt auseinander: Der Kritiker moniert, dass Hoppe ihr 'Talent zu unterhaltsamer Darstellung' nicht nutze und “"ein ungeheures Potenzial” verschenke, indem sie sich "zügellos egozentrischen Selbstbetrachtungen" hingebe, eine "bei näherem Hinsehen zutiefst unliterarische Attitüde", [...] die immer wieder zur Ausschließung “williger Leser” führe'. ${ }^{749}$ Die Passage enthält die zentrale Fragestellung zu Lesbarkeit in Bezug auf das Life Writing: wie wirkt sich der Fokus auf die eigene Person auf die Leser aus? ${ }^{750}$ Die oben zitierte fingierte Kritik suggeriert, dass 'Hoppes' Idiosynkrasie sowohl der Unterhaltungsaspekt als auch das Erreichen einer potenzial vorhandenen und interessierten großen Leserschaft zum Opfer falle. Die Nabelschau exkludiert demnach aufgrund der narzisstischen Autorschaftsauffassung den Leser und führt zum Entzug des literarischen Status. Es kann durchaus angezweifelt werden, dass sowohl der Ausschluss der Leser als auch die Einordnung zutreffen. Zum einen werden dabei die Bedürfnisse der Leserschaft außer Acht gelassen, der es etwa nach einem voyeuristischen Vergnügen am Leben der Autorin oder aber auch nach einer intensiven

\footnotetext{
747 Ebd., S. 75.

748 Ebd., S. 123.

749 Ebd., S. 116.

750 Zum Life Writing vgl. u.a. Lee 2009 sowie Smith/Watson 2010. Zum Life Writing im Kontext der Narratologie siehe Löschnigg 2010.
} 
Auseinandersetzung mit der Funktionsweise der Fiktionalisierung verlangen könnte. Zum anderen wirkt bereits die Einordnung als Roman dem an eine Autobiographie erinnernden Titel Hoppe entgegen und verweist somit auf Literarizität.

$\mathrm{Zu}$ Beginn des Romans gibt Hoppe den Lesern eine Lektürehilfe, die die Funktionsweise des Romans anzeigt. Sie thematisiert 'die Frage nach der Authentizität', wobei sie sich dezidiert darauf bezieht, was sie tatsächlich selbst erlebt hat und was reine Erfindung ist:

Ständig wirft [Hoppe] Köder aus, um sie kurz darauf wieder einzuholen, bevor der Fisch seinen Haken findet. Ein Verfahren, das ihren Lesern bis heute abwechselnd Freude und Ärger bereitet: 'Man war drinnen und bleibt doch draußen', bemerkt früh ein verprellter Kollege.

Man gäbe ihm recht, würde man nicht auf den zweiten Blick sofort erkennen, dass Hoppes Werk die Unterscheidung von drinnen und draußen weder kennt noch sucht, dass der schroffe Ausschluss nicht kalkuliert, [...] sondern ehrliche Selbstbeschreibung ist. ${ }^{751}$

Die Stelle korrespondiert mit Hoppes poetologischer Auffassung der Leserschaft, da sie auf eine positive Indifferenz gegenüber dem Publikum verweist. Letztlich bleibt die Entscheidung, sich auf das Werk einzulassen oder nicht, beim Leser, wodurch ein egalitäres Verhältnis hergestellt wird. Dass Hoppe tatsächlich Köder auswirft, die zudem auf eine breitgefächerte Leserschaft zielen, spiegelt sich ebenfalls in dem Abschnitt. Denn er stellt unterschiedliche Rezeptionsweisen in Aussicht. Erstens kann der Leser das Spiel der Autorin mit ihrer Identität vernachlässigen und den Roman zum reinen Vergnügen lesen. Dazu baut Hoppe die oben analysierten Vereinfachungsstrategien ein, die eine leichte Lesbarkeit sichern. Allerdings fungieren dieselben Mittel ebenfalls als Köder für eine intellektuell fundierte Auseinandersetzung mit dem Roman. Die vermeintliche editorische Seriosität und Hoppes Koketterie mit dem Genre der Autobiographie eröffnen dem Leser im Spannungsfeld mit der Gattungsbezeichnung 'Roman' ein vielfältiges Potenzial, sich selbst kreativ mit dem Text auseinanderzusetzen. Somit liefert der Roman diverse Anschlusspunkte für vielfältige Publikumsgeschmäcke.

Der Vergleich zwischen Hoppe und Iwein Löwenritter zeigt, dass Hoppe im Kinderwerk tatsächlich eine größere Freiheit im Umgang mit Lesbarkeit pflegt. In beiden fungieren Genrekonventionen dazu, Lesbarkeit zu legitimieren, jedoch auf 
unterschiedliche Art. Im Löwenritter eröffnen sie ihr die Möglichkeit, das dynamische Potenzial des Lesbarkeitsbegriffs gründlich auszuschöpfen, wobei sie das Genre als hochliterarisches ansieht. In Hoppe dient der recht statische editorische Trick ebenfalls der Sicherung und Legitimation von Lesbarkeit, allerdings verspricht sie sich daraus einen Mehrwert, der Lesbarkeit durch eine vermeintliche Verkomplizierung und intellektuelle Anreicherung als Mittel der Erwachsenenliteratur zulässig macht.

\title{
Ulrike Draesner
}

Während Hoppe sich im Lesbarkeitsdiskurs in den Traditionen der mittelalterlichen Epik, des Märchens und der Kinderliteratur positioniert, stellt Ulrike Draesner ihre Autorpoetik in die aristotelische Mimesistradition. Im Folgenden werden die Auswirkung dieses Anschlusses an die Tradition auf ihre Einstellung zu Lesbarkeit und auf deren Umsetzung im Roman Sieben Sprünge vom Rand der Welt analysiert werden.

In ihren Bamberger Poetikvorlesungen Zauber im Zoo (2007) diskutiert Draesner die Wirkungsabsicht der Belehrung in Bezug auf Horaz und die antike Rhetorik und Poetik:

\begin{abstract}
Literatur soll, so die Tradition, docere et delectare. Allerdings gibt es, gerade was Unterhaltung angeht, Effektiveres. Schon die docere-und-delectare-Römer ergötzten sich lustvoll in blutigen Arenen realer Grausamkeit. Und schon damals wurde, als die Triebe des Menschen regulierendes Gegen-Projekt, die 'hohe Literatur' entworfen: zähmen sollte sie, hemmen, humanisieren. Erziehung des Menschengeschlechtes. [...] Dennoch: unterhalten werden und etwas über die Welt erfahren sind weiterhin die beiden Pole zwischen denen literarische Texte sich entfalten..$^{752}$
\end{abstract}

In ihrem Rückgriff auf die horazische Tradition unterscheidet Draesner zwischen 'Literatur' und der metaphorisch qualitativ übergeordneten 'hohen Literatur'. Im Kontext des Begriffs 'Literatur' klassifiziert sie docere und delectare als ebenbürtige Wirkungsabsichten, dem Begriff der 'hohen Literatur' hingegen ordnet sie ausschließlich eine erzieherische Funktion zu. Somit ist Unterhaltung zwar ein inhärenter Aspekt von Literatur im Allgemeinen, aber nicht mit Prestige verbunden. Abschließend betont sie die Bedeutung des Spannungsfeldes zwischen Unterhaltung 
und Didaktik in der Gegenwart, das sie durch die traditionelle Einordnung unmittelbar an Qualitätsfragen knüpft. Das movere spricht sie hier nicht an.

Zumindest auf die Funktion der Gefühlsregung rekurriert Draesner an zwei Stellen in ihren Vorlesungen. So macht sie geltend, dass Literatur durch Sprache und Motive, 'nicht unseren Verstand, sondern vor allem unsere Gefühle in Bewegung setzt. ${ }^{753}$ Der Verstand kann zum docere in Bezug gesetzt werden, das Draesner der Anregung der Gefühle unterordnet. Diese benennt sie explizit als Intention für ihre eigenes Schaffen: 'Mich interessiert eine sachliche Erzählkunst, kombiniert mit Gefühlen, die das Erzählen zeigt und erzeugt. Mich interessiert das Zusammenkommen äußerer und innerer Wirklichkeit' ${ }^{754}$ Draesner verbindet einen künstlerischen Anspruch mit dem Erzählen, das sie dadurch als ansprechende literarische Form positioniert. Die Betonung der 'Wirklichkeit' lässt auf einen realistisch inspirierten und daher der Lesbarkeit zugetanen Literaturbegriff schließen. ${ }^{755}$

In ihren Vorlesungen legt Draesner dezidiert ihre Definition des 'Realismus' dar, indem sie sowohl auf die historische Epoche als auch auf die poetologische Strategie eingeht:

Zum einen meint Realismus eine diffus bestimmte literarische Epoche. [...] [Zum anderen meint] 'Realismus': Mimesis, Nachahmung, Abbildung der Wirklichkeit. Doch was soll das heißen? Abbildung nach welchen Regeln? Alle Autoren, aller Zeiten, versprechen immer wieder: mit der gekurvten Wirklichkeit so um eine Kurve zu fliegen, dass die Kurvenbiegung des Erzählens identisch damit ist, wie die Wirklichkeit ganz ohne zu fliegen als kurvig Wahrgenommene sich selbst bei sich ins Zeug, nämlich in die Kurve legt. Immer wieder spielen Autoren, Bücher und Leser damit. Und wir wissen, dass das Fiktion ist, dass die Autoren wissen, dass es Fiktion ist, und die Autoren wissen, dass wir wissen, dass sie wissen etc. Dennoch unternehmen wir-Sie-ich das Spiel, von Wirklichem zu erzählen, erneut. Es scheint sich zu lohnen. ${ }^{756}$

Während die Epoche im historischen Kapitel der vorliegenden Arbeit am Beispiel Theodor Fontanes exemplarisch analysiert wurde, ist für die Lesbarkeitdebatte der Gegenwart Draesners zweite Bedeutung des Realismus relevant, bei der sie sich auf die aristotelische Mimesis-Tradition bezieht. Draesner steht dem Prinzip der Mimesis

\footnotetext{
753 Ebd., S. 51.

754 Ebd., S. 105.

755 Vage bleibt die Funktion des Adjektivs 'sachlich'. Sollte es sich um einen Verweis auf die Strömung der 'Neuen Sachlichkeit' handeln, dann spräche dies - wie im historischen Kapitel am Beispiel Erich Kästners beobachtet - für eine poetologische Nähe zur Lesbarkeit.

756 Ebd., S. 86.
} 
kritisch gegenüber. Sie hinterfragt insbesondere die Methodik der Abbildung der Wirklichkeit und verweist auf die zur Aufrechterhaltung einer solchen Annahme nötige suspension of disbelief. Dennoch ist sie der Ansicht, dass der Realismus in der Gegenwart eine prominente Position im deutschen Literaturbetrieb einnimmt und als literarisches Erfolgsmodell gilt, dem die Lohnmetaphorik implizit eine kommerzielle Komponente zuordnet. Draesners Ausführungen können demnach als Indiz dafür gelten, dass sich das seit den 1990er Jahren in der Lesbarkeitsdebatte eingeforderte Realismusdesiderat in der deutschen Literatur etabliert und zudem den Wunsch nach Erfolg erfüllt hat. ${ }^{757}$

Draesner entwirft ein genaues Bild von der gegenwärtigen deutschen Literaturlandschaft und diskutiert zudem die Rolle des Autors in diesem Kontext. Als Ausgangspunkt wählt sie die deutsche Nachkriegsliteratur seit den 1950er Jahren:

[Damals bildete sich] das unsere Nachkriegs-Literaturlandschaft bis heute formende Biotop, in dem Erwartungen an Literatur und Autorenrollen abgesteckt sind. [...] Was in Deutschland Nachkriegsliteratur hieß, ist vorbei. Doch wann hörte der Nachkrieg auf? Die Kriegsfolgejahre gingen über in Jahrzehnte eines Nachkrieges des Nachkrieges. Wir brauchten sie. Erst nun, im neuen Jahrtausend, mit dem Sterben der jüngsten Täter und Opfer des Nationalsozialismus, könnte auch dieser zweite Nachkrieg sich abschließen. Es ist Zeit, dies zu übersetzen: in unsere Themen, Bilder und Sprachen $[\ldots] .{ }^{758}$

Draesner nutzt eine historische Gegebenheit zu einer Reevaluierung der Literatur. Ähnlich wie die Kritiker der 'Gesinnungsästhetik' die Wende nutzten, um gegen eine politische und moralische Didaktik in der deutschen Literatur vorzugehen, nimmt Draesner das allmähliche Ableben der Zeitzeugen des Nationalsozialismus als Anlass, um über die deutsche Literaturlandschaft und Literatur zu reflektieren. Dabei hebt sie drei Punkte besonders hervor: die Rolle der Autoren, die an die Literatur gestellten Erwartungen und die Auswirkungen der zeitgeschichtlichen Hintergründe auf die literarische Produktion.

\footnotetext{
757 Wie Kehlmann und Hoppe verbindet Draesner mit der realistischen Gestaltung eine Vorliebe für das Phantastische, was sie wie Aristoteles dadurch in den Mimesisbegriff integriert, dass die phantastischen Element in der dargestellten Welt plausibel sind. Daher ergeben sich im Hinblick auf Lesbarkeit keine Schwierigkeiten: 'Unmögliches möchte möglich erscheinen [...]. Zauberer ist, wer uns schweben lässt, wie Eis schwebt zwischen Wasser und Luft. Er behauptet ein "als ob" als "als ob", und macht es auf eben diese Weise wirklich. Nicht Dinge verwandelt er, sondern uns. Er ist ein Teil unserer Sehnsucht nach dem Realen, das nicht als real gilt, doch wirklich ist. Eben das ist Literatur.' (Ebd., S. 46f).

758 Ebd., S. 30f.
} 
Hinsichtlich der Rolle des Autors zieht Draesner Kafkas Erzählung 'Im Dunkel der Gasse' (1923) heran. Sie interpretiert Kafkas Fragment als Metaerzählung über die Genese eines Textes und geht insbesondere auf die Figur des grünen Drachen ein:

Liest man Kafkas kurzen Text als Parabel auf das Entstehen eines Textes, erzählt er, dass an diesem Prozess mindestens drei Kräfte beteiligt sind: der Autor 'ich', der Text als das, als das er erschienen ist (Drache) und als das, was er noch nicht ist, aber erzeugt - als Idee seiner selbst, als Vision dessen, was er werden könnte. ${ }^{759}$

Für die Analyse des Lesbarkeitsbegriffs ist vor allem von Bedeutung, wen Draesner nicht in den Entstehungsprozess eines Werkes einbezieht - den Leser. Text und Autor nehmen gegenüber dem Leser, dem Endpunkt der literarischen Produktion, eine Vorrangstellung ein. Dies ist nicht mit Draesners Äußerung, durch Literatur wirkungsästhetisch Gefühle erregen zu wollen, kompatibel. Sie hält sich jedoch den Spielraum offen, den Leser in diese Gleichung einzuführen, was sie später im SiebenSprünge-Projekt umsetzt.

Draesner führt dann in poetischer Form textexterne Faktoren an, die ihre Arbeit maßgeblich beeinflussen. Diese spiegeln die im Literaturstreit geäußerte Sorge um den Erfolg der deutschen Literatur:

Den Schreibprozess und seine Voraussetzungen der Umgebung abtrotzen. Auch dem eigenen Ich. Das auch in diesem Sinn VOR dem Text ist:

vor den eigenen Ängsten: was geschieht mit mir

vor der ständigen Frage: wie lebe ich davon, wie komme ich durch

unsicher, ohne Absicherung

vor einem sich brutalisierenden Buchmarkt, der großen

Autorenshow, wachsender Marginalität

vor innerer anstrengender Arbeit. ${ }^{760}$

Draesner greift die Problematik der mentalen und physischen Anstrengungen des Schreibprozesses sowie der kommerziellen Voraussetzungen des Schreibens auf. Der Ausdruck biographisch fundierter Emotion in Form der Angst um die Sicherung des Existenzminimums etabliert wie bei Krausser den Wunsch nach kommerziellem Erfolg als für den Schreibprozess relevante textexterne Kategorie. Diese Sorgen bedingen die Entwicklungen auf dem Literaturmarkt. Ähnlich wie Politycki, Wittstock und Hielscher erkennt Draesner eine zunehmende Konkurrenzsituation auf dem Markt, die es einem

\footnotetext{
759 Ebd., S. 40.
}

760 Ebd., S. 50. 
einzelnen Autor schwer macht, zu reüssieren. ${ }^{761}$ Im Zuge dessen spricht sie ebenso wie Hoppe ein vermehrtes Drängen der Autoren in die Öffentlichkeit an. Die Fokussierung des Event-Charakters impliziert ebenso wie der Wunsch nach Verkaufserfolg einen poetologischen Einfluss auf die Textgenese. Allerdings kann laut Draesner die Eventisierung den sukzessiven Bedeutungsverlust der Literatur und der Autoren in der Gesellschaft nicht aufhalten.

Die Entwicklung hin zur Marginalität begründet Draesner mit einer allgemeinen gesellschaftlichen Entwicklung, Menschen zunehmend nach ihrem ökonomischen Erfolg zu beurteilen. Dabei stellt sie in ihrer Argumentation dezidiert die Abhängigkeit der Literatur von zeitgeschichtlichen Kontexten heraus, die sich ebenso wie im Lesbarkeitsbegriff dynamisch auf die vorherrschenden poetologischen Parameter auswirken können:

Auch der Schreibprozess hat [...] eine Außenseite, die Ökonomie heißt. Das mag schon immer so gewesen sein - das einzige überlieferte Dokument zum Leben Walthers von der Vogelweide etwa [...] handelt davon, dass Waltherus cantor [...] eine würdige Gabe, nämlich Geldsumme für einen Pelzmantel, empfangen habe. Der Stellenwert dieser Außenseite allerdings verändert sich. In einer Sozietät, die mit dem Ende eines sogenannten Kalten Krieges ihr gesellschaftlich-ideologisches Gegenbild verloren hat, das von anderen Werten als jenen des Marktes und seiner 'freien Wirtschaft' auszugehen suchte, wächst aus Bequemlichkeit oder mangels Alternativen die Neigung, Menschen zunehmend danach zu bewerten, wie viel Geld sie erzeugen bzw. die anderen kosten.

Literarisches Schreiben beruht auf einer komplexen Gemengelage innerer und äußerer sowie innerer mit inneren Lebensfaktoren, die mit den beiden Begriffen 'Inspiration' und 'Handwerk' nur grob umrissen wären. Die Vorstellung von Originalgenies und kostenlosen Musenküssen, anziehend in ihrer Einfachheit und dienlich der Mystifizierung des Schreibprodukts als unkritisierbar und autonom, ist längst obsolet. [...] Man weiß, dass ein ganzes Leben dahinter steht.

Nichts anderes gilt für den Autor. Ein Text schreibt sich daraus, wie man lebt, und was man darin und dadurch ist. Förderungen, Preise und Stipendien tragen dazu bei, die innere Einstellung auf das Schreiben hin aufrecht erhalten und umsetzen zu können. Dies bedeutet andersherum, dass man, wenn man Fördergelder streicht, die Erfindungsräume von Texten empfindlich einschränkt. ${ }^{762}$

Wie Hoppe verhandelt Draesner eine Spannung zwischen dem Geniegedanken und den Marktbedingungen am Beispiel von Walther von der Vogelweide. Wie ihre Kollegin verabschiedet sie sich von der Vorstellung des autonomen Genies und bettet den Autor in die Umstände seiner Zeit. Dies macht sie für die gesamte Tradition deutscher

761 An anderer Stelle zeigt sich zudem, dass Draesner damit auch, entsprechend den vom Programm der 'Neuen Deutschen Lesbarkeit' analysierten Marktbedingungen, die zunehmend internationalen Markteinflüsse meint; vgl. ebd., S. 27.

762 Ebd., S. $53 f$. 
Literatur geltend, wobei sie jedoch die Gegenwart aufgrund ihrer speziellen, aus dem Fall des Ostblocks resultierenden, sozio-ökonomischen Bedingungen hervorhebt. Sie integriert die Kunst in den Kontext allgemeinen Gewinnstrebens, wobei finanzieller Erfolg allen anderen Qualitäten, im Falle der Literatur künstlerisch ästhetischen, den Rang abläuft. In diesem Rahmen gerät die Literatur als wenig gewinnbringendes Medium, das stark von finanziellen Zuwendungen abhängig ist, ins Abseits. Draesner dekonstruiert die Vorstellung eines gegenüber dem Publikum exponierten Originalgenies und betont die literarische Produktion als laboröse und alle Lebensbereiche umfassende Tätigkeit, die egalitär zu anderen Professionen und daher auch zu ihren Lesern in den gesellschaftlichen Kontext eingegliedert ist.

Hatte Draesner sich zuvor kritisch gegenüber Literaturevents und den Marktprozessen gezeigt, so kommt es zum Ende der Passage zu einer Art Versöhnung, die einem Abgesang auf die idealistische Vorstellung Hegels vom 'freien poetischen Kunstwerk' gleichkommt. Draesner erkennt an, dass der Betrieb durch Gelder und Events entscheidend dazu beiträgt, die literarische Produktion am Laufen zu halten, und zudem kreative Freiheiten sichert. Hierin ähnelt ihre Auffassung der von Glavinic, der ebenfalls davon ausgeht, dass eine solide finanzielle Absicherung zur Qualitätserhaltung beiträgt. Vom Literaturbetrieb geschaffene Institutionen wie 'Förderungen', 'Preise' und 'Stipendien' - und diese Liste ließe sich noch durch weitere Events wie Lesereisen, Writer-in-Residence-Programme oder Poetikprofessuren erweitern - übernehmen dabei die Funktion eines modernen Mäzenatentums.

Darüber hinaus beobachtet Draesner wie Wittstock, dass die Konkurrenzsituation zwischen der Literatur und den neuen Medien, deren Entstehung sie im Kontext des Mauerfalls ansiedelt, zum Beliebtheitsschwund der deutschen Literatur in der öffentlichen Meinung beiträgt:

In Deutschland folgte eine Kanondebatte, müde, weil sich unsere nach 1989 frei ausgreifend globalisierten und mediatisierten Großgesellschaften nur mehr marginal über literarische Medien definierten.

Und heute? Die Zeiten literarisch affirmativer Feierlichkeitskultur versus littérature engagée, die Zeiten deutscher 'Innerlichkeit' und in lange Bärte nuschelnder Poeten sind glücklicherweise vorbei, doch auch die langsam akklimatisierte Postmoderne [...] weist Schwächen auf, und Popliteratur und Fräuleinwunder gar sind so schnell verschwunden, wie sie erschienen waren.

Wo also stehen wir? Filme, Spiele, Tausende von Songs, Abertausende von Bildern von noch mehr Websites, second-life-Entwürfe und Identitäten, 
Bedienungsmodi von Handys, Fernsehern, Playstations, Planern, Note- und Subnotebooks wirbeln, neben Büchern, durch unsere Köpfe. ${ }^{763}$

Während die Forschung dem Literaturstreit als Diskussion um die Rolle der Intellektuellen in der Gesellschaft eine eminente öffentliche Relevanz zuschreibt, spielt Draesner die Bedeutung des Literaturstreits herunter. Diesen Reflex begründet sie damit, dass die deutsche Literatur im Zuge der Globalisierung von den neuen Medien in eine gesellschaftliche Randstellung gedrängt wurde. Dabei ist Draesners Liste weitaus vielfältiger als die Wittstocks, worin sich die rapide technische Entwicklung spiegelt. Draesner unternimmt eine Bestandsaufnahme der deutschen Gegenwartsliteratur. Folgt man ihren Ausführungen, so ist der Literaturstreit dahingehend einflussreich gewesen, dass die politisch engagierte Literatur der Nachkriegszeit in allen ihren systemaffirmativen und -kritischen Ausprägungen zu einem Ende gekommen ist.

Ebenso sieht Draesner das Ende einer Literatur gekommen, die sich ausschließlich auf das aus der Gesellschaft zurückgezogene Ich konzentriert, und erkennt eine direkte Auseinandersetzung mit gesellschaftlichen Themen in der Literatur. ${ }^{764}$ Zudem begrüßt sie das sich wandelnde Bild von Autoren. Die Hervorhebung der Bärte stellt eine Kritik an der genderspezifischen Vorstellung des Autors als altem Mann dar, außerdem suggeriert das Nuscheln ein Defizit in der öffentlichen Selbstdarstellung und Präsentation des Werks. Zuletzt attestiert Draesner der Postmoderne Defizite, die einen Epochenwandel einleiten könnten. Als Erscheinungsformen der Postmoderne führt sie die im Zuge der Lesbarkeitsdebatte entstandenen Strömungen der Popliteratur und des Fräuleinwunders an. Anders als Politycki, der die beiden in Die Farbe der Vokale wegen eines vermeintlichen Qualitätsmangels kritisiert, klassifiziert Draesner Popliteratur und Fräuleinwunder ohne qualitative Wertung als temporäre Modeerscheinungen und stellt sogleich die Frage nach einem literarischen Paradigma der Gegenwart.

In den Vorlesungen kommt sie zu folgendem Ergebnis, in dem sich essenzielle Aspekte des Literaturstreits und des Programms der 'Neuen Deutschen Lesbarkeit' abzeichnen:

[Ich] beobachte eine merkwürdige Spaltung. Man erwartet von Autoren Romane, am liebsten große, und $\mathrm{zu}$ Recht Auseinandersetzungen mit Zeitgeschichte, Lebenswandelwandel, Wissenschaft etc. Seit in den 1990ern das deutsche Erzählen

763 Ebd., S. 57.

764 Vgl. dazu auch Wittstock 1995, S. 91-113. 
vorgeblich zurückkehrte, seit dann aber auch diverse Pop-Stufen verpufften, sind die Erwartungen auf frühe Moderne gestellt. Nicht zu viel Experiment, nicht zu viel Verunsicherung. Kohärente fiktive Welt [...]. Andererseits erwartet man von Autoren ein zeitgenössisch bewegliches, medienerfahrenes Leben. ${ }^{765}$

$\mathrm{Zu}$ Beginn verdeutlicht Draesner, dass sie unter der Abkehr von der Innerlichkeit eine literarische Auseinandersetzung mit dem Zeitgeschehen versteht. Die Hervorhebung der Wissenschaft kann zudem als Verarbeitung von Bildungsgut gesehen werden. Die Betonung der Renaissance des Erzählens deutet auf einen Erfolg der Etablierung von Lesbarkeit als literarisches Paradigma. Draesner erkennt die Moderne als Bezugspunkt, die im Literaturstreit von Greiner, Schirrmacher und Bohrer als Qualitätsgarantin ins Feld geführt worden war. Dies trifft auch auf Draesner zu, da sie den Rückgriff auf die Moderne als Reaktion auf die vermeintlich qualitativ minderwertigere Popliteratur etabliert. Draesner wählt jedoch einen Begriff von moderner Literatur, der auf Lesbarkeit deutet, da er neben dem Qualitätsprädikat der Moderne auch ein Desiderat nach leichtem Textverständnis impliziert. Kohärenz spricht für eine stringente, leicht nachzuvollziehende Handlung. Der Wunsch, den Leser nicht zu verunsichern, steht für eine auf eine möglichst angenehme Lektüre ausgerichtete Textgestaltung. Es bleibt noch die zweite von Draesner ausgemachte Erwartung zu klären: die der Kompetenz im Umgang mit den Neuen Medien. Zwar sieht sie die Literatur in einem Konkurrenzverhältnis zu diesen, lamentiert jedoch nicht darüber, sondern macht sich die Neuen Medien aktiv zu Nutzen. Dies zeigt sich am deutlichsten am Beispiel ihres bislang letzten Projekts, Sieben Sprünge vom Rand der Welt. ${ }^{766}$ Bewusst wurde hier die Formulierung Projekt gewählt, da Draesners Werk die Welt des Romans transzendiert und dadurch interessante neue Fragestellungen in Bezug auf Lesbarkeit aufwirft.

Mit Sieben Sprünge vom Rand der Welt verfolgt Draesner eine ambitionierte Agenda: zum einen wählt sie die Vertriebenenthematik der deutschen Nachkriegsgeschichte als Sujet, zum anderen verhandelt sie diese mit Hilfe der komplexen Forschungsgebiete der Primatenforschung und des transgenerationalen Traumas. Beide wissenschaftlichen Komplexe nutzt sie zudem, um auf der Ebene der histoire verschiedene Formen der Nachahmung zu diskutieren. Interessanter ist Draesners Roman jedoch im Hinblick auf

765 Ebd., S. 55.

${ }^{766}$ Zuvor hatte sich Draesner z.B. bereits an dem Online-Projekt neuedichte.de beteiligt. 
die multimediale Strategie, deren Analyse aufzeigen kann, welche neuen Formen der Lesbarkeit im gegenwärtigen Kontext hervorgebracht werden könnten. Denn Draesner liefert mit ihrem Sieben-Sprünge-Online-Projekt einen interessanten Beitrag dazu, wie die neuen Medien das im Zuge der Lesbarkeitsdebatte formulierte Ziel, die Literatur vermehrt in den öffentlichen Raum zu tragen, forcieren könnten. Dabei wird zu beobachten sein, dass durch Draesners transmedial angelegtes Projekt die Beziehung zwischen Autor und Leser rekonfiguriert wird.

Die Sieben Sprünge sind mit einer komplexen Erzählkonstruktion gebaut. Die Erzählung umspannt vier Generationen, verknüpft das Schicksal zweier Flüchtlingsfamilien und deckt dabei eine Zeitspanne ab, die vom Ersten Weltkrieg bis hin zur Gegenwart des Jahres 2014 reicht. Nach einem kurzen in der auktorialen dritten Person erzählten Prolog, der noch vor der Flucht der Familie Grolmann aus Breslau angesiedelt ist, wechselt Draesner in den elf darauf folgenden Kapiteln zwischen neun verschiedenen Ich-Erzählern, die sowohl aus der Familie Grolmann als auch aus der Familie Nienalt stammen, wobei die Erzählung springend zwischen mehreren Zeitebenen und Schauplätzen wechselt. Aus der Familie Grolmann fungieren folgende Personen als Erzähler: Lilly und Hannes (Großeltern, decken die Zeit vom ersten Weltkrieg bis in die ersten Jahrzehnte in der BRD ab), Eustachius und Emil (deren Söhne, Zeit der Flucht bis, im Falle von Eustachius, in die Gegenwart hinein), Simone (Eustachius' Tochter, geboren 1962) und Esther (Simones Tochter, geboren 1996). Aus der Familie Nienalt erzählen Halka (Großmutter, ab der Flucht aus der Ukraine bis zur Zeit um Boris' Geburt 1966), Boris, deren Sohn, und Jennifer (Boris’ Tochter, geboren Anfang der 1990er). Simone und Lilly erzählen jeweils zweimal. Die Übergänge zwischen den einzelnen Kapiteln werden jeweils durch die Angabe des jeweiligen Erzählers zu Beginn angezeigt. Die Distinktion wird weiterhin (außer bei den Erzählfiguren Halka und Emil) durch ein ihnen zugeordnetes, aus der Affensprache Yerkish entlehntes, Symbol angedeutet.

Zwar werden die Erzähler in vorhergehenden Kapiteln mal mehr, mal weniger prominent in Bezug auf ihr Verhältnis zu der jeweils erzählenden Figur eingeführt, jedoch bewirkt die multiperspektivische Erzählhaltung, die eine durchgehende Handlungsführung verhindert, einen hohen Schwierigkeitsgrad. Draesner wechselt oft, bis auf die Benennung der Erzählinstanz unmarkiert, zwischen Zeitebenen und Schauplätzen in den ehemaligen Ostgebieten und der BRD. Eine unmittelbare 
chronologische und räumliche Verortung der Handlung ist daher oftmals schwer möglich und stiftet beim Leser zunächst Verwirrung. ${ }^{767}$ Während in Hoppes Löwenritter oder in Glavinics Das größere Wunder Sprünge entweder klar markiert, chronologisch nachvollziehbar und/oder in der Erzählstruktur motiviert waren, ergibt sich der Übergang bei Draesner erst im Laufe des jeweils nachfolgenden Kapitels. So endet beispielsweise die erste Lilly-Erzählung mit einem Zeit- und Ortswechsel und den ihr als Flüchtling in Bayern entgegengebrachten Vorurteilen. Die Gegenwartsebene von Simone beginnt dann mit einer Bergwanderung von Simone und ihrer Tochter Esther, die wiederum nicht, wie z.B. bei den Wechseln der Erzählebenen in Glavinics Roman, chronologisch und thematisch direkt an die vorhergehende Simone-Erzählung anschließt. 768 Die Sieben Sprünge folgen demnach keiner stringent aufbauenden Handlung, sondern kreisen um das Vertriebenensujet, das sich durch alle präsentierten Generationen zieht und den Roman thematisch zusammenhält. Auf der Gegenwartsebene wird dieses durch die wissenschaftlichen Disziplinen sowie durch den emotional familiären Zugang der Charaktere gefiltert. Der Roman ist somit in seiner Struktur äußerst komplex angelegt. Er bietet jedoch dem Leser durch das Sujet und die Positionierung der Charaktere zum Sujet einen roten Faden an, der ihn durch den Roman leitet, wobei es dem Leser selbst überlassen wird, sich die Bezüge klarzumachen.

Hinsichtlich der sprachlichen Gestaltung setzt Draesner der Komplexität vereinfachende Strategien gegenüber. Als Beispiel kann die in lyrischer Form gehaltene folgende Passage gelten:

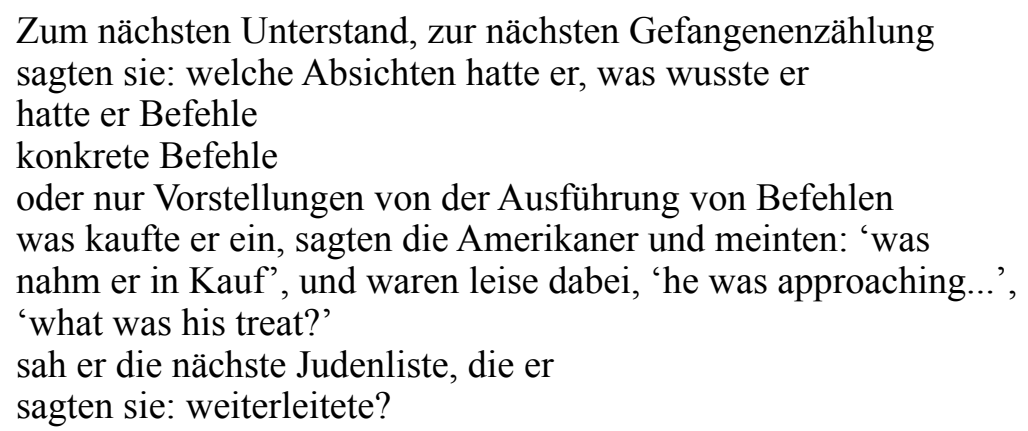

767 Die Erzählung springt folgendermaßen: Prolog zur Kriegszeit im Osten, 1., 2. und 3. Kapitel Simone, Boris und Eustachius in der Gegenwart, 4. Kapitel Lily von der Kriegszeit bis ins Frühjahr 1945, 5. Kapitel Simone in der Gegenwart, 6. Kapitel Hannes post mortem von den Erfahrungen im Ersten Weltkrieg bis in die ersten Jahrzehnte der Bundesrepublik, 7. Kapitel Jennifer in der Gegenwart, 8. Kapitel Lilly im München der Nachkriegszeit ebenfalls bis in die frühen Jahrzehnte der BRD, 9. Halka in Breslau zu Kriegszeiten bis hin zu Boris' Geburt 1966, 10. Kapitel Esther in der Gegenwart und 11. Kapitel Emil auf der Flucht in Sondershausen im Frühjahr 1945.

768 Vgl. Draesner 2014 k, S. 232f und 67f. 
oder nahm er Gefangene im nächsten Dorf, gab er den Befehl, die Juden auszusortieren, stellte er dafür Soldaten ab

zur nächsten: 'Singstrecke'

was sah er auf der Strecke, die er abfuhr

was hieß er gut, indem er nichts tat, he didn't say a word

not a word did he say

'utter' sagten sie

und, sagten sie: mit welcher Absicht, in welchen Zustand, 'of

your mind'

sagten sie, 'fuhren Sie am nächsten Morgen weiter', 'you',

wenn er wusste, dass er an dem Nackten, der ein Judenlied singen

musste, schon vorbeigekommen war

oder

wenn

der Platz am Morgen leer lag, selbstverständlich

leer, das Ende der Holzstange auf dem Boden ruhte, aufruhte,

das blutige Ende, sagten sie

am Boden, am Grund. ${ }^{769}$

Die gesamte Passage besteht aus einem einzigen, komplexen und aus Enjambements zusammengesetzten Satz. Deutlich erkennbar sind Gestaltungsmittel der Lyrik wie der an eine Anapher gekoppelte Parallelismus zu Beginn oder die chiastische Stellung von 'he didn't say a word / not a word did he say', was die starke künstlerische Aufbereitung der Stilistik unterstreicht. Während die Sprachrichtigkeit und das - mit Ausnahme der englischen Passagen - einfach zu verstehende Vokabular das Verständnis erleichtern, wird dies wiederum erschwert durch Satzlänge und Komplexität der Syntax sowie die reduzierte Verwendung von Interpunktion. Auch wenn inzwischen davon ausgegangen werden kann, dass ein Großteil der deutschsprachigen Leserschaft kompetent genug ist, um die von Draesner in einfachem Englischen gehaltenen Einschübe zu übersetzen, bleiben sie doch jenem Teil der Leserschaft, der des Englischen nicht so mächtig ist, unverständlich. ${ }^{770}$ Andererseits handelt es sich bei dem Rückgriff auf das Englische um eine realistische Gestaltungsweise, die der Lebenswirklichkeit der deutschen Bevölkerung und der amerikanischen Besatzer am Ende des Zweiten Weltkriegs entspricht. Mehrsprachigkeit ist hier eine Facette des Realismus, die nicht für Lesbarkeit spricht, indem gewisse Gruppierungen des Lesepublikums vom leichten Verständnis ausgeschlossen werden.

\footnotetext{
769 Ebd., S. 368; das Zitat bemüht sich das Druckbild des Originals möglichst genau nachzuahmen.

770 Diese sind beispielsweise bei weitem nicht so lang und so komplex wie Thomas Manns fremdsprachige Passagen im Zauberberg. Als Beispiel kann das komplett auf Französisch geführte Gespräch zwischen Hans Castorp und Clawdia Chauchat gelten, vgl. Mann 2013, S. 465-473. Zum anderen muss hier auch noch angefügt werden, dass Draesners Roman stellenweise konkret mit den Sprachproblemen der Flüchtlinge spielt und die Besonderheiten des Deutschen und Polnischen und die Ergebnisse von Mischformen der beiden Sprachen ausstellt, vgl. u.a. Draesner 2014 k, S. $417 f$.
} 
Allerdings fungiert eine realistisch inspirierte Sprache vor allem auf der Gegenwartsebene als ausgleichendes Gewicht zu den komplexeren Stellen. Z.B. greift Draesner auf umgangssprachliche Wörter wie das von dem Moderator Stefan Raab geprägte Modewort 'Schland' zurück, um am Beispiel von Boris' Tochter Jennifer sowohl deren Sportbegeisterung als auch das seit der Fußball Weltmeisterschaft 2006 mit dem Begriff verbundene Nationalbewusstsein in Deutschland auszudrücken. ${ }^{771}$ Dann lässt sie Simones Tochter Esther nach Hause 'radel[n]', was unmittelbar den süddeutschen Sprachraum und das Setting München evoziert. ${ }^{772}$ Beide Begriffe sind Ausdruck der mimetischen Einbettung der Romanfiguren in die Gegenwart, welche dem Leser einen unmittelbaren Bezug zu den Figuren herstellen und deren Verortung in seiner eigenen Lebenswirklichkeit zulässt. ${ }^{773}$

Auch in Bezug auf das komplexe akademische Sujet wendet Draesner Vereinfachungsstrategien an, die auf ein Lesbarkeitsdesiderat hindeuten. Als paradigmatisch kann Simones Einführungsvorlesung in die Thematik der Primatenforschung herangezogen werden:

\begin{abstract}
Meine Damen und Herren, gute Nacht.
Ich möchte Ihnen hier im Dunkel des Hörsaals etwas Dunkles zeigen. Ich habe Sie eingeladen, mit mir über Grundlagen der Primatenforschung nachzudenken. Hell strahlt, heißt es, das Licht der Vernunft. Der Mensch, die Krone der Schöpfung, steht ihm am nächsten, nur das Auge Gottes, des vernünftigen Wesens schlechthin, dessen Ebenbild der Mensch ist, so Thomas von Aquin, Augustinus, Leibniz und viele andere, schwebt über ihm. Es nährt sein Gehirn. Wir sind, sagen wir, helle im Kopf. Wir brauchen Licht, um zu leben, ebenso brauchen wir Dunkelheit. Dunkel ist unser Körperinneres. Dunkel das tierische Leben. Dunkel sind auch unsere Ursprünge. Dorthin möchte ich heute mit Ihnen blicken. [...] Von den Menschenaffen her. ${ }^{774}$
\end{abstract}

Mit dem ersten Satz eröffnet Draesner in einer überraschenden Abwandlung der gängigen Begrüßungsformel 'guten Tag' den Kontext der Nacht, den sie in die modifizierte Dichotomie zwischen Tag und Nacht, bzw. Licht und Dunkel ausarbeitet. Das Licht etabliert sie im Verbund mit dem Adjektiv 'hell' als Zeichen der Vernunft und legt damit bereits eine Opposition zum animalischen Charakter des Menschseins an. Die

\footnotetext{
771 Zur Begriffsgeschichte vgl. Leurs 2014; zur Verwendung des Begriffs im Roman siehe Draesner 2014 k, S. $416 f$. 772 Ebd., S. 523.

773 Auch wenn Draesner in ihren Poetikvorlesungen keinen Anspruch darauf formuliert, muss im Bezug auf die international ausgerichtete Breitenwirkung durch Lesbarkeit hier die Frage gestellt werden, inwieweit solch spezifisch deutschen bzw. lokalen sprachlichen und kulturellen Konventionen durch Übersetzung ins Ausland transportiert werden können.

774 Ebd., S. 114.
} 
Zuteilung des Lichts zu der Vernunft ist leicht nachzuvollziehen, der gebildete Leser wird die Lichtmetapher zudem in den Kontext der Aufklärung als Widersacherin der Theologie (siehe auch Englisch: enlightenment) stellen und im Sinne der postmodernen Doppelkodierung Vergnügen aus dieser Erkenntnis ziehen. Die theologische Basis leitet Draesner mit der Generalmetapher vom Menschen als Krone der Schöpfung ein, die als weit verbreiteter Terminus gelten kann und den Leser deswegen vor keine Verständnisschwierigkeiten stellt - dies gilt auch für die Phrase 'helle im Kopf', die zudem die Zuteilung von Licht und Geist/Verstand im Gegensatz zum Körperlichen untermauert. Durch die eindeutige Unterteilung ist die Position der herangezogenen Instanzen wie Thomas von Aquin klar, der bewanderte Leser wird hingegen durch den Abgleich mit theologischem Fachwissen zusätzlich unterhalten. Das Dunkel bezieht Draesner unmittelbar auf die Primaten und damit auf das Tierische. Als Scharnier zwischen den Bereichen Licht und Dunkel verweist sie dezidiert auf die eröffnete Dichotomie und bezieht die Dunkelheit umgehend wieder auf das Körperliche, was eine konsequente Untermauerung der Zuteilungen darstellt und dem Leser das Nachvollziehen des Gedankengangs erleichtert. Sie beschließt die Stelle mit dem Rückbezug auf den Anfang, indem sie Nacht und Primaten wiederum in Bezug setzt. Zusammen mit der zumeist wenig komplizierten syntaktischen Gestaltung durch Parataxen und im letzten Teil auch mit der analeptischen Einleitung der Sätze (die dreimalige Verwendung von 'Dunkel' am Satzbeginn) sichert Draesner mit der eindeutigen Zuteilung und semantischen Aufladung der Hell-Dunkel-Dichotomie die Lesbarkeit und Verständlichkeit des Textes, was an die von Hoppe im Iwein zur leichten Orientierung eingesetzte Farbgebung erinnert.

Zudem integriert Draesner ein popkulturelles Gegengewicht zu den komplexen wissenschaftlichen Fragestellungen in den Roman, was als Mittel zur Unterhaltung einer breiten Leserschaft angesehen werden kann. An zwei Stellen nutzt sie J.K. Rowlings Harry-Potter-Reihe als Referenzsystem. In beiden Fällen wird der intertextuelle Verweis von Erzählerinnen aus der jungen, mit den Harry-Potter-Romanen aufgewachsenen Generation herangezogen, was zum einen für eine realistische Erzählweise spricht und zum anderen auch einer jungen Generation von Lesern Identifikationspotenzial bietet. In Jennifers Kapitel dienen die sich bewegenden und interagierenden Gemälde der Zauberschule Hogwarts dazu, die Nutzung des sozialen 
Netzwerks Facebook zu erklären. ${ }^{775}$ Jennifer etabliert die Potter-Referenz als Mittel, das es ihr im Gegensatz zum für sie schwer nachzuvollziehenden psychologischen Ansatz des Vaters ermöglicht, im Hinblick auf soziale Strukturen Zugang zu den Funktionsweisen von und den kommunikativen Interaktionen auf Facebook zu finden. Dadurch wird ein komplexes System durch eine generationenübergreifend weitbekannte und besonders bei einer jungen Generation von Lesern populäre Referenz anschaulich gestaltet. Durch die detaillierte Erklärung des Wesens der Zaubergemälde bleibt das Konstrukt zudem auch für die nicht mit den Potter-Romanen vertrauten Leser verständlich. Auch die zweite Harry-Potter-Stelle dient der Veranschaulichung. Esther nutzt den im dritten Potter-Band eingeführten Patronus-Zauber zur Erschließung bestimmter Verhaltensweisen ihres Großvaters. ${ }^{776}$ Erneut wird die Referenz ausreichend erklärt, um Nicht-Potteranern das Verständnis der Stelle zu ermöglichen, und wiederum fungiert sie als Mittel zur anschaulichen Erklärung komplexer Vorgänge.

Wie innerhalb der Diegese die Potterstellen kann textextern die Website < dersiebte-Sprung.de> als Instrument gelten, den Lesern die im Roman abgebildete Welt zu veranschaulichen. Laut Ankündigungstext auf der Website basiert jene auf der Grundidee, 'einen Bogen zu schlagen und das Buch zu öffnen.' ${ }^{777}$ Das Buch wird als alleiniger Textträger abgelöst. Die Website fungiert als gleichberechtigter Teil eines Projekts, was auch die Titelgebung der Website ausdrückt. Der Roman macht sechs Sprünge aus - gemeint sind die Erzählungen von sechs Erzählern, die nach gegenwärtigem deutschen Recht als Flüchtlinge gelten -, die Website fungiert als letzter Sprung, der das Projekt abrundet. Die Zusammengehörigkeit der beiden Teile des Projekts zeigt im Buch ein nach dem Ende des Haupttextes angeführter Verweis auf die Website. Einerseits ist dort ein QR-Code abgedruckt, der per Smart-Phone-Scan unmittelbar zur Website führt und so eine direkte Verbindung zwischen Roman und Netz herstellt. Andererseits entwirft Draesner in lyrischer Form ein Programm für die Website:

Rückseite. Making of.

Fadenverlauf. Verworfenes, Entstehungsdaten, 'Fakten' wie gesehen, begriffen, verzogen, gefühlt. Der letzte

Sprung.

775 Vgl. ebd., S. 411.

776 Vgl. ebd., S. 504.

777 Draesner 2014 a. 
Sprechraum, Stimmenort. ${ }^{778}$

Dem 'letzten Sprung' werden zwei Funktionen zugeordnet. Die erste kann unter dem Begriff Bonusmaterial zusammengefasst werden. Dazu passt der ebenfalls aus dem Filmbereich stammende Terminus des 'Making of', das die Hintergründe zum Hauptfilm liefert. Für Lesbarkeit ist die Metapher des Fadenverlaufs relevant, die darauf deutet, dem Leser durchgehend eine Orientierungshilfe zur Seite zu stellen. Ähnlich verhält es sich mit den angeführten historischen Fakten. Durch die Darstellung des Wie erhält der Leser Einblick in die Konstruktion des Romans, die ihn den Text leichter nachvollziehen lassen. Die zweite Funktion der Website ist es, einen Ort des Austausches zu schaffen. Es handelt sich um die Förderung des Dialogs zwischen Autor, Leser und Text.

Bei der ersten Funktion lassen sich drei unterschiedliche Methoden zur Veranschaulichung des Romans ausmachen. Zunächst betrifft diese wortwörtlich die Visualisierung von im Roman beschriebenen Elementen. Als Beispiel kann zum einen die Verlinkung eines im Roman beschriebenen Videos der BBC gelten, in dem eine Gruppe Affen des Kannibalismus überführt wird. ${ }^{779}$ Zum anderen finden sich auf der Website zahlreiche Fotos der Schauplätze des Romans, die aus dem Zeitraum um das Kriegsende 1945 stammen. ${ }^{780}$ In beiden Fällen findet durch eine konsequent mimetische Darstellung eine Fusion von textbasierenden und visuellen Medien statt, die dem Leser dabei helfen, sich von den Geschehnissen der Romanebene ein Bild zu machen. Draesner selbst äußert im auf der Website enthaltenen Interview:

Also nachprüfbare Realitätsschnipsel einzuarbeiten, die die Frage nach dem Verhältnis dieser Wirklichkeit zur Fiktion noch einmal aufwerfen. Das gilt immer wieder in diesem Roman: in Scherben von Daten und Namen ragt die 'Wirklichkeit' in den Text. ${ }^{781}$

Dies spricht für eine mimetische Darstellung, die der Lesbarkeit förderlich ist. Jedoch reicht dies nicht aus, um dem Prozess gerecht zu werden, den die Website im Zusammenspiel mit dem Roman auslöst. Denn es ist nicht nur so, dass die Wirklichkeit in den Text ragt, sondern der Text ragt über die Website auch in die (virtuelle) Realität.

\footnotetext{
778 Draesner 2014 k, S. 557.

779 Vgl. ebd., S. 40-43, sowie Draesner 2014 b.

780 Vgl. Draesner 2014 c und Draesner 2014 f.

781 Vgl. Sieben Fragen 2015.
} 
Z.B. finden sich zwischen Draesners Darstellung des Kannibalismus und dem tatsächlichen Video Unterschiede. Das gefressene Affenbaby etwa fällt im Video der Mutter nicht aus dem Fell, sondern wird bei der Flucht von der Herde getrennt. Durch diese Diskrepanzen diffundiert die Wirklichkeit nicht nur in den Roman, sondern der Roman diffundiert auch in die Wirklichkeit. Es wird ein ständiger Prozess der Projektion und Rejektion in Gang gesetzt, in dem die beiden Versionen der Wirklichkeit gegeneinander ausgespielt werden. Dadurch wird dem Leser trotz der realen Quellen ein kreatives Potenzial bei der Rezeption des Textes zugestanden, indem ihm die Autorin anbietet, die Spannungen zwischen den Realitätsebenen selbst zu lösen.

Die zweite Methode zielt darauf, der Leserschaft das wissenschaftliche Sujet zugänglich zu machen. Auf der Website legt Draesner ein 'Lexikon der reisenden Wörter' an, das zudem ein weiteres Beispiel dafür ist, dass der Roman in die Wirklichkeit diffundiert, da es laut Website der Figur Esther gehört. ${ }^{782}$ Die meisten darin aufgelisteten Einträge sind außerdem auf den Vor- und Nachsatzseiten des Romans abgedruckt. ${ }^{783}$ U.a. werden für das Verständnis des Romans bedeutende Begriffe der Primatenforschung sowie der Psychologie - z.B. 'Pan troglodytes schweinfurthii' oder 'Postmemory' - im Lexikon ausführlich erklärt. ${ }^{784}$ Dadurch wird auf der Website die Verständlichkeit des Romantexts gefördert.

Die letzte Methode wirkt der Komplexität der Erzählstränge entgegen. In einem auf der Website veröffentlichten Essay, erörtert Draesner ihre Herangehensweise an das komplexe Sujet der Vertreibungen und legt dar, wie sie selbst Zugang zu dem Thema fand: 'Ich musste einen multi-logischen Roman schreiben. Multi-logisch in der doppelten Bedeutung des Wortes: verschiedenen Lebenswahrheiten folgend, von verschiedenen Seiten her gesprochen.' ${ }^{785}$ Draesner liefert eine Lektürehilfe, die die Struktur des Romans offenlegt und als Rezeptionsanleitung gelesen werden kann. Dennoch ist dem Terminus 'multi' Komplexität inhärent. Es geht besonders um die multiperspektivische Erzählweise des Romans, die im Falle der Sieben Sprünge dem aristotelischen Postulat nach einem stringenten Handlungsverlauf widerspricht. Zusätzlich gibt Draesner ihren Lesern in der Rubrik 'Es spricht' eine weitere Lektürehilfe zur Hand, die dem Leser den Faden zur Verfügung stellt, um sich zwischen

\footnotetext{
782 Vgl. Draesner 2014 e.

783 Draesner nennt dies bezeichnenderweise ‘im Übergangsbereich zwischen Buch und Nichtbuch'; vgl. ebd.

784 Vgl. Draesner 2014 i und Draesner 2014 g.

785 Vgl. Draesner 2014 d.
} 
den verschiedenen Erzählebenen zurechtzufinden. Draesner erstellt zu jedem ihrer Erzähler eine Extraseite, auf der sie neben einer Textstelle aus den betreffenden Kapiteln jeweils einen Familienstammbaum der Erzählerfigur angibt. Die Stammbäume sind in die beiden Familien Grolmann und Nienalt unterteilt, sodass die Figur umgehend einer der beiden zugeordnet werden kann. Dies erleichtert die Erschließung der Konstellationen zwischen den einzelnen Erzählfiguren und lässt sie unmittelbar einer der beiden am Ende konvergierenden Flüchtlingsgeschichten zuordnen. Um den genauen zeitlichen und lokalen Hintergrund verständlich zu machen, stellt Draesner zudem Geburtsdatum und Ort der Figuren zur Verfügung, wodurch sich die Übersiedelung von Ost nach West anhand der Generationenfolge nachzeichnen lässt. Als letztes werden die Erzählerfiguren von den als 'Nebenfiguren' bezeichneten visuell abgehoben, sodass eine unmittelbare Privilegierung erkennbar ist. ${ }^{786}$ Durch diese einfach gestalteten und prägnanten Hilfsmittel werden die komplexeren Zusammenhänge der Erzählung für den Leser leicht zugänglich dargestellt.

Der Leser ist die zentrale Komponente der zweiten Funktion der Website. Denn Draesner versucht explizit, eine Plattform zu schaffen, auf der Leser etwaige Flüchtlingsgeschichten veröffentlichen können. 'Erzählen Sie selbst!' lautet die Idee der Rubrik:

Der Roman heißt 'Sieben Sprünge vom Rand der Welt', obwohl nur sechs Vertriebene ihre Geschichte erzählen. Jeder von ihnen ist vom Rand seiner Welt gesprungen. [...]

Hier in der Rubrik 'Selbst-Erzählen' ist Platz für Ihre Gedanken zu Flucht und Vertreibung, Ihre Erfahrungen mit dem Thema in verschiedenen Generationen, Ihre Geschichte(n), Ihre Leseeindrücke. Auch Fragen an die Autorin sind willkommen. Und neue Worteinträge für das 'Lexikon der reisenden Wörter' werden auf Bestellung geliefert. ${ }^{787}$

Hier wird eine Kommunikation zwischen Autorin, Leser und Text in Gang gesetzt. Der Verweis auf die Beantwortung von Fragen und das Lexikon demonstriert ein Verständnisdesiderat, das ein aktiver Dialog gewährleisten soll, bei dem spezifisch auf die Bedürfnisse des individuellen Lesers eingegangen wird. Ein gänzlich neuer Aspekt ist der des 'Selbst-Erzählens'. Durch die Aufforderung der Autorin an den Leser, sich an der Gestaltung des Projekts zu beteiligen, kommt es zu einer Rekonfiguration der

\footnotetext{
786 Vgl. Draesner 2014 h.
}

787 Draesner 2014 j. 
Beziehung zwischen Schreibenden und Lesenden. Die Autorin setzt den Leser als egalitären Partner bei der Vervollständigung des Werks ein. Die Geschichten der Leser stehen als ebenbürtige Pendants zu der Geschichte des Romans. Dies steht zum einen im Gegensatz zu Draesners poetologischer Äußerung, dass sie den Leser bei der Textgenese ausblendet. Zum anderen spricht dies insofern für den nachhaltigen Erfolg der Lesbarkeit, als sich, wenn auch nicht durchgehend im literarischen Text, ein leichtes Textverständnis als Ziel manifestiert und die Position des Lesers gegenüber der des Autors aufgewertet wird.

Mit ihrem Sieben-Sprünge-Projekt beschreitet Draesner neue Wege. Anders als das Programm der 'Neuen Deutschen Lesbarkeit', das die neuen Medien überwiegend als Konkurrenz ansah, macht sie sich diese zur Umsetzung von Lesbarkeit zu Nutze. Durch den transmedialen Charakter des Projekts löst sie das Buch als alleinigen Textträger ab die Grenze zwischen Text und Paratext wird durch die gleichwertige Behandlung der Website als Teil eines Gesamtprojekts fluide. ${ }^{788}$ Dem Leser kommt eine vergleichende Rolle zu. Der Text transzendiert die Ebene des Buches und wirkt durch die Website auf die Leser, die wiederum rückwirkend aktiv den Text mitgestalten können.

Hoppes und Draesners Werke bestätigen den poetologischen Einfluss von Lesbarkeit auf die deutsche Literatur im frühen 21. Jahrhundert. Im Vergleich zu ihren Kollegen Krausser, Kehlmann und Glavinic stehen sie ihr differenzierter gegenüber und ergreifen nicht aktiv für sie Partei, suchen jedoch auf kreative Weise nach Wegen, um in ihren literarischen Arbeiten auf sie zurückzugreifen. Hoppes Iwein Löwenritter und Hoppe sowie Draesners Sieben Sprünge vom Rand der Welt operieren fluide zwischen Genres bzw. Medien, erweitern so den Anwendungsbereich von Lesbarkeit und verweisen so auf die Analysevielfalt, die es benötigt, um den Lesbarkeitsbegriff umfassend zu eruieren, sei es durch die Anwendung etablierter Theorien oder durch die Erschließung neuer Referenzsysteme.

\footnotetext{
788 Der Begriff 'Paratext' soll im Sinne von Gérard Genettes entsprechender, in Seuils (1987) dargelegter, Theorie verstanden werden. Genette definiert Paratexte als alle den Haupttext begleitenden und repräsentierenden Texte - wie u.a. Titel oder Kapitelüberschriften -, die einen Text für die Leser erst als Buch zugänglich machen. Dabei macht auch er die Existenz der in der vorliegenden Arbeit bei der Analyse des Sieben-Sprünge-Projekts beobachteten hybriden Formen von Haupttext und Paratext geltend. Vgl. Genette 1987, v.a. S. 7-19.
} 


\section{Perspektiven}

Die vorliegende Arbeit hat gezeigt, dass Lesbarkeit in der Krisenzeit des Literaturstreits im Kontext der Auseinandersetzung um die zukünftige Ausrichtung der deutschen Erzählliteratur eine eminente Bedeutung als Lösungsansatz zur Krise erlangte. ${ }^{789} \mathrm{Um}$ diese Entwicklung nachzuvollziehen, wurde der Lesbarkeitsbegriff nach seiner theoretischen Grundlegung und nach der Analyse seiner Tradition in Bezug auf die deutsche Erzählliteratur verankert. ${ }^{790}$ Von dieser Basis ausgehend konnte Polityckis Auffassung von der 'uralten' Lesbarkeit bestätigt werden. Bei der insbesondere von Autoren vorangetriebenen Etablierung von Lesbarkeit als Paradigma handelt es sich demnach um eine Problemlösung, die Thomas S. Kuhn wie folgt definiert: "problems can be solved with conceptual and instrumental techniques close to those already in existence.' ${ }^{791}$ Unter Bezug auf fünf Autoren, die im Zeitraum seit dem Literaturstreit mit bedeutenden Romanen hervorgetreten sind, wurde untersucht, welche Signifikanz die Auseinandersetzung mit Lesbarkeit für die literarische Praxis entwickelt hat. Diese Konstellation verweist auf das für Lesbarkeit konstitutive Zusammenspiel von Theorie, zeitgeschichtlichem Hintergrund und literarischer Praxis.

Dennoch stellen die 1990er Jahre in der deutschen Literatur eine Wendephase in Bezug auf Lesbarkeit dar, die den im Rahmen des Literaturstreits in den Vordergrund gerückten Begriff der Lesbarkeit aus seinen früheren Erscheinungsformen heraushebt. Die historische Zäsur der Wiedervereinigung veränderte die deutsche Literaturlandschaft tiefgreifend. Die intensiviert kompetitiven Marktbedingungen führten zu einer Dominanz insbesondere englischsprachiger Autoren, aufgrund derer sich diverse Vertreter des deutschen Literaturbetriebs gezwungen sahen, sich produktiv mit den neuen Verhältnissen auseinanderzusetzen. Dabei avancierte die angloamerikanische Literatur zum Vorbild für poetologische Lösungsansätze. Der vor allem von Daniel Kehlmann als Maßstab herangezogene Jonathan Franzen dokumentiert in seinem Essayband How to be alone (2002), wie er in den 1990er Jahren zu einer tiefgreifenden Einsicht kam, die ihn dazu bewegte, einen ästhetischen Wandel zu vollziehen: 'I intend this book [...] as a record of a movement $[\ldots]$ toward an acceptance

\footnotetext{
${ }^{789}$ Kuhn zufolge ist ein Paradigmenwechsel eine von drei Antworten auf Krisenzeiten. Jener könne nur über den Kampf um die Akzeptanz eines neuen unter Überwindung eines alten Paradigmas herbeigeführt werden; vgl. Kuhn 1996 , S. 84.

790 Zur Möglichkeit eines solchen Paradigmas vgl. ebd., S. 86.

791 Ebd., S. 96.
} 
- even a celebration - of being a reader and a writer.' ${ }^{792}$ Franzen zeichnet hier eine Entwicklung, die die deutsche Literatur im Zuge der Lesbarkeitsdebatte noch zu vollziehen hatte: Eine strikte Trennung der Positionen des Autors und des Lesers wird überwunden. Der Autor zieht Inspiration aus einem egalitären Verhältnis zwischen den beiden, demzufolge der Autor beim Verfassen der eigenen Texte auch seine Funktion als Leser wahrnimmt und beide Positionen in Personalunion vereint. Dadurch kommt es zwischen Autor und Leser zu einem fruchtbaren Austausch der Bedürfnisse, der eine hierarchische Vorrangstellung des Autors zusehends obsolet macht. Draesners SiebenSprünge-Projekt legt nahe, dass der Leser in einem solchen Prozess der Annäherung und Gleichstellung außerdem zunehmend Einfluss auf die Textgenese erhält. ${ }^{793}$ Hierin zeigt sich vor allem auch die zunehmend signifikante Rolle der sozialen Medien und des Internet, deren Potenzial und Wirkung in dieser Hinsicht noch zu erschließen sind. ${ }^{794}$

Für deutsche Schriftsteller bedeutet dies nicht nur die Nähe zum nationalen Publikum, sondern explizit auch den Anschluss an eine internationale Leserschaft. Breitenwirkung wird nun auf einem globalen Literaturmarkt angestrebt; für dieses Bestreben schufen die politischen, ökonomischen, sozialen und kommunikativen Entwicklungen nach dem Ende des Kalten Krieges eine praktische Basis. Die ästhetische Basis mussten sich die Autoren erst selbst erarbeiten, und sie wandten sich zunächst sowohl der klassischen Mimesis-Tradition als auch Vorbildern aus dem Ausland zu. Zuvor war Lesbarkeit als Mittel zum Zweck akzeptabel gewesen, so etwa bei Wieland, bei der Gartenlaube oder auch in Bechers Literaturprogramm als Garantin zur Erziehung einer breiten Masse. An sich wurde ihr aber im Vergleich zu komplexen literarischen Formen die Qualität abgesprochen. Seit den 1990er Jahren und speziell zu Beginn des 21. Jahrhunderts jedoch wurde sie in der deutschen Literaturszene als eigenständige literarische Qualität mit einer primären Unterhaltungsfunktion etabliert.

Den Prozess zur Etablierung der Lesbarkeit um die Jahrtausendwende leiteten Autoren ein, die literaturpolitisch für sie eintraten. Die Vertreter der Lesbarkeit mussten

\footnotetext{
792 Franzen J. 2010, S. 5f.

793 Es stellt sich zudem die Frage nach der didaktischen Funktion. Im Zuge des angeführten Prozesses scheint man von einer explizit didaktischen Funktion von Literatur abgekommen zu sein. Jedoch zeigt sich insbesondere bei den in den beiden vorhergehenden Kapiteln besprochenen Autoren der Anspruch, beispielsweise Bildungsgüter an den Leser zu vermitteln und jenen zu einer intellektuellen Auseinandersetzung mit dem Gelesenen zu bewegen, was auf eine zwar weitaus subtilere jedoch weiterhin erzieherische Funktion der Literatur hindeutet.

794 In diesem Kontext lohnt auch ein Blick auf die immer populärer werdende Form der Fan Fiction, die in anderen Medien bereits erheblichen Einfluss auf die Primärtexte genommen hat. Ein Beispiel sind etwa die Fanreaktion auf den Cliffhanger der BBC-Serie Sherlock (2010); vgl. dazu Opp 2016.
} 
sich sowohl diachronisch mit der älteren Generation als auch synchronisch mit der 'Neuen Moderne' auseinandersetzen, um sich dann später von der nach Meinung von Polyticki und Krausser qualitativ minderwertigen deutschen Popliteratur abzugrenzen, ehe der Paradigmenwechsel in einer Art darwinistischem Kampf um ein 'Survival of the Fittest' Lesbarkeit zumindest vorübergehend als zentralen literarischen Bezugspunkt etablierte. $^{795}$

Für die Bestimmung eines Paradigmenwechsels macht Kuhn die folgenden beiden Merkmale geltend:

First, the new candidate must seem to resolve some outstanding and generally recognized problem that can be met in no other way. Second, the new paradigm must promise to preserve a relatively large part of the concrete problem-solving ability that has accrued to science through its predecessors. Novelty for its own sake is not a desideratum [...]. ${ }^{796}$

Im Literaturstreit fürchteten beide Parteien um die Zukunft der deutschen Literatur unter den neuen globalen Marktbedingungen und entwickelten jeweils ein mehr oder weniger fundiertes poetologisches Programm, um die deutsche Literatur für die neuen Herausforderungen im In- und Ausland konkurrenzfähig zu machen. Während die Bemühungen der 'Neuen Moderne' um einen Anschluss an die Literatur des frühen 20. Jahrhunderts sowohl in der Theorie als auch in der Praxis scheiterten, gelang es der 'Neuen Deutschen Lesbarkeit' und ihren Nachfolgern graduell eine Poetologie zu entwickeln, die einen nachhaltigen Einfluss auf die Literaturproduktion in Deutschland haben sollte. Sie beriefen sich vornehmlich auf poetologische Parameter der aristotelischen Mimesis-Tradition, der klassischen Rhetorik und der gegenwärtigen internationalen Erzählliteratur, die - wie im theoretischen Kapitel dargestellt - in einem für Lesbarkeit konstitutiven dynamischen Wechselspiel aufeinander wirken. Auf diese Weise konnten die Vertreter der Lesbarkeit auf der Basis einer prestigeträchtigen Tradition zuvor qualitativ wenig geltende Kategorien wie leichten Stil und Unterhaltung privilegieren.

Anhand dieser theoretischen Grundlage unternahmen es die deutschsprachigen Autoren Krausser, Kehlmann und Glavinic, die mit der Paradigmenstellung der

795 Kuhn erkennt vor allem beim Abschluss große Parallelen zwischen seiner Paradigmen- und Darwins Evolutionstheorie: 'the resolution of revolutions is the selection by conflict within the scientific community of the fittest way to practice future science'; Kuhn 1996, S. 172.

796 Ebd. S. 169. 
Lesbarkeit verbundenen Gestaltungsmöglichkeiten in ihren Romanen auszuloten. Ein Entdeckungspotenzial ist ein inhärenter Bestandteil von Kuhns Paradigmentheorie. Dieser bemerkt, dass ein akzeptiertes neues Paradigma noch sehr vage in seiner Ausformulierung sein kann, sodass eine umfassende Theorie und Praxis von seinen Vertretern erst erschlossen werden muss; zudem hebt er hervor, dass den Anhängern eines Paradigmas bei einem grundsätzlichen Übereinkommen über dessen Grundsätze viele Wege zu dessen Ausgestaltung offenstehen. ${ }^{797}$ Kuhn zieht die Metapher einer Landkarte heran: ein Paradigma fungiert als Lösungsansatz eines Problems und als Instruktionshilfe dazu, weitere Entwicklungen der Disziplin anzuregen und deren theoretische Festlegung zu unterstützen. ${ }^{798}$

Während es die vorliegende Arbeit vornehmlich unternommen hat, den poetologischen Begriff der Lesbarkeit mit einem in der Tradition gründenden theoretischen Analysemodell zu versehen, konnten die vielfältigen Facetten des Lesbarkeitsbegriffs nur anhand weniger Beispiele aus der Gegenwartsliteratur angedeutet werden. Das Trio Krausser, Kehlmann und Glavinic wurde als Beispiel für eine konkrete literaturpolitische Zusammenarbeit gewählt, die sich die Etablierung der Lesbarkeit als ästhetische Qualität zum Ziel setzte und deren literarische Werke sich genügend unterscheiden, um diverse praktische Gestaltungsmöglichkeiten der Lesbarkeit sichtbar werden zu lassen. Die Untersuchung zur Poetik und literarischen Praxis von Hoppe und Draesner diente weiterführend dazu, anhand ihrer Poetiken Lesbarkeit als sich etablierendes Paradigma im deutschen Literaturbetrieb zu bestätigen - wobei die Autorinnen zumindest aufgrund ihrer poetologischen Vorbehalte als advocatae diabolae bezeichnet werden können. Mit ihren kreativen Herangehensweisen an Lesbarkeit relativieren sie jedoch diese poetologischen Vorbehalte, liefern Beispiele für Wege der Legitimation lesbarer Texte und erkunden weitere ästhetische Gestaltungsmöglichkeiten.

Um ein erweitertes Bild der durch Lesbarkeit eröffneten Schaffensräume zu erhalten, müsste ein weitaus größeres Korpus in Betracht gezogen werden, als es im Rahmen dieser Arbeit möglich war. Neben der Sichtung der Vielzahl an deutschen Publikationen in der Gegenwart böten sich u.a. die folgenden Forschungsbereiche an, um die Lesbarkeitspraxis weiter $\mathrm{zu}$ erschließen. Die Analyse von weiteren 
Autorkollaborationen und die Erforschung des inspirativen Austausches zwischen Autoren könnte sowohl weiterführende Erkenntnisse über literaturpolitische Strategien als auch Einblicke in den interkulturellen Spielraum der Lesbarkeitsdebatte liefern. Beispielsweise scheint sich Krausser in seinem Romanwerk intensiv mit Ian McEwan auseinanderzusetzen. Der Roman Eros etwa könnte hinsichtlich seines Sujets und seiner strukturellen Anordnung potenziell als unmittelbare Antwort auf McEwans Atonement (2001) gelesen werden. Generell könnte eine Ausweitung der komparatistischen Komponente auf literarische Kulturen jenseits der anglo-amerikanischen Tradition tiefgreifende Erkenntnisse über die Struktur des globalen Literaturmarktes und der vorherrschenden Ästhetiken zu Tage fördern sowie weiterführende Erkenntnisse in Bezug auf einen möglichen universellen Charakter der Lesbarkeit liefern.

Aber auch im Kontext der deutschen Literatur gilt es, ein vertieftes Verständnis für die Bedeutung von Lesbarkeit zu gewinnen. So wären in Bezug auf die Tradition weitere Identifizierungen von historischen Erscheinungsformen, sowie eine Analyse der praktischen Umsetzung der im historischen Kapitel besprochenen Poetologien erstrebenswert. Für den Zeitraum der Nachkriegszeit bedarf es einer kritischen Untersuchung der älteren Autorengeneration um Günter Grass und Martin Walser, die mit dem Begriff der 'Gesinnungsästhetik' in Verbindung gebracht wurde. Im Hinblick auf die Literatur seit der Wende wären die ob ihres Verkaufserfolgs häufig im Kontext der 'Neuen Deutschen Lesbarkeit' rezipierten Strömungen der Popliteratur und des 'Fräuleinwunders' zu analysieren. Besondere Aufmerksamkeit verdienen in der Erarbeitung eines Lesbarkeitsparadigmas besonders solche Fälle, die Kuhn 'conversions' nennt; die Betrachtung von Mitgliedern des Literaturbetriebs, die sich von einer der Lesbarkeit oppositionellen Position abgewandt haben, könnte tiefere Einsichten in die Mechanik des Paradigmenwechsels ergeben. ${ }^{799}$ Als literarisches Beispiel könnten etwa Christian Kracht mit seinem Roman Imperium (2012) fungieren, als theoretisches Beispiel Ulrich Greiners Leseverführer. 800

Eine weitere 'conversion', die in der vorliegenden Arbeit bereits angeführt wurde, betrifft die Literaturrezeption. Durch eine strategische Zusammenarbeit, die auf Verlage, Kritik und Germanistik zielte - wie sie etwa Krausser, Kehlmann und Glavinic pflegen

\footnotetext{
799 Vgl. ebd., S. 159.

800 Aufgrund von Übereinstimmungen bei Sujet und Ästhetik böte sich etwa ein Vergleich von Walsers Roman Ein liebender Mann (2008) und Krachts Imperium (2012) mit Kehlmanns Vermessung an.
} 
- konnte erreicht werden, dass sich auch diese Institutionen zu einem jeweils unterschiedlichen Grad aktiv mit Lesbarkeit befassten und deren Einfluss als positive Entwicklung für die deutsche Erzählliteratur bewerteten und förderten.

Kuhn verbindet mit einer Paradigmenstellung keinerlei qualitative Wertung. Im Prinzip sei ein Paradigmenwechsel ein Umschwung, der weder bessere noch schlechtere Standards nach sich zieht, sondern einen neuen Lösungsansatz für bestimmte Problemstellungen schafft. ${ }^{801}$ Im Falle der Etablierung von Lesbarkeit als dem literarischen Paradigma der deutschen Literatur im frühen 21. Jahrhundert soll jedoch in der vorliegenden Untersuchung ausdrücklich von einer positiven Entwicklung gesprochen werden. Denn sie ermöglichte es der deutschen Literatur in einer Krisenzeit, die neuen Marktbedingungen und die Chancen der neuen Medien aktiv zu nutzen und ästhetische Ansätze zu entwickeln, die einen aktiven Dialog mit dem breiten Publikum suchen. Denn die Aufwertung von Lesbarkeit eröffnete den ambitionierten Autoren die Möglichkeit, das in der Einfachheit liegende kreative Potenzial zu erschließen, ohne sich qualitativer Vorbehalte erwehren zu müssen.

Zudem entstanden im deutschen Literaturbetrieb seit den frühen 1990er Jahren neue Institutionen, die das Publikum enger an die Literatur banden und binden. Die Literaturhäuser wurden in den späten 1980er Jahren gegründet, schlossen sich jedoch erst Ende der 1990er wirksam funktional zusammen, um das 'Literaturhaus als Synonym für eine zeitgemäße, wandlungsfähige Förderung und Vermittlung von deutschsprachiger und internationaler Gegenwartsliteratur weiter zu verbreiten.' 802 Auffällig ist zunächst die dezidiert internationale Ausrichtung der Literaturhäuser, was auf die Globalisierung der Literaturen hindeutet und für die deutschsprachige Literatur die Konkurrenz mit anderen Literaturen sichtbar macht. Die Literaturhäuser suchen neue Wege, um sich den Anforderungen der Gegenwart zu stellen, wobei Breitenwirkung einen inhärenten Bestandteil von deren Programm darstellt. ${ }^{803}$ Ebenso divers aufgefächert wie die kulturellen Hintergründe der geförderten Werke ist das Zielpublikum der Literaturhäuser: 'Schriftsteller, Verleger, Übersetzer, Lektoren,

\footnotetext{
801 Vgl. Kuhn 1996, S. 108.

802 Literaturhäuser 2015.

803 Dass hiermit auch explizit der finanzielle Aspekt von Literatur verbunden ist, zeigt eine spätere Passage aus dem Einführungstext auf der Website: 'Veranstaltungen mit renommierten Künstlern oder experimentierfreudigen jungen Autoren bieten Unternehmen eine interessante Chance, sich im Kultursponsoring zu engagieren. Angestrebt ist eine Partnerschaft, die beide, den Wirtschaftsfaktor der Kultur und den Kulturfaktor der Wirtschaft, gleichermaßen widerspiegelt.' (Ebd.).
} 
Buchhändler, Kulturschaffende, Journalisten und literaturbegeistertes Publikum begegnen sich im offenen und freien Dialog. ${ }^{904}$ Es zeigt sich die Vorstellung eines ebenbürtigen Verhältnisses zwischen allen Beteiligten des Literaturbetriebs. Ein besonderes Augenmerk liegt auf dem allgemeinen Publikum, das als gleichberechtigter Dialogpartner fungiert und damit ebenso Einfluss auf den literarischen Diskurs nimmt wie das Fachpublikum. ${ }^{805}$ Das Beispiel der Literaturhäuser verdeutlicht, dass im Literaturbetrieb seit 1990 zusehends der Prozess eines Wechselspiels zwischen Schaffenden und Rezipierenden eingeleitet wurde, in dem der Leser eine zunehmend prominente Rolle erhält. Abschließend heißt es auf der Website der Literaturhäuser hinsichtlich deren Funktion:

Die Literaturhäuser fungieren dabei als Katalysatoren. Vielfältige Anregungen aus dem urbanen Umfeld fließen in die Programmgestaltung ein. Literaturhäuser spiegeln nicht nur aktuelle Strömungen, sondern geben selbst weitreichende kulturelle Impulse an den Kulturbetrieb und die Öffentlichkeit weiter. ${ }^{806}$

Die Literaturhäuser erfüllen eine Vermittlerfunktion zwischen Publikum und Produzierenden. Dabei wird den Lesenden eine ebenso bedeutende Rolle bei der Gestaltung des literarischen Diskurses zugesprochen wie den Schreibenden. Der Austausch zwischen beiden führt dann in aktiver Zusammenarbeit zur Impulsgebung in beiden Gruppen. Diese Symbiose steht für das von der Lesbarkeitsdebatte angestrebte egalitäre Verhältnis zwischen Schreibenden, Produzierenden und Rezipienten.

Ein weiteres Instrument, um die Aufmerksamkeit für die Literatur in der Öffentlichkeit zu steigern, ist der seit 2005 verliehene Deutsche Literaturpreis, der in seiner Vergabestruktur am Beispiel des britischen Booker Prize modelliert wurde. Der Buchpreis erfüllt laut seiner offiziellen Website folgende Funktion: 'Ziel des Preises ist es, über Ländergrenzen hinaus Aufmerksamkeit zu schaffen für deutschsprachige

\footnotetext{
804 Ebd.

805 Auch dieses wird als möglichst breit und vielfältig angesehen: 'Die Literaturhäuser fördern in ihrer Arbeit einen Diskussionsprozess, in dem ganz verschiedene gesellschaftliche Gruppen Gelegenheit zum Gedankenaustausch haben. So gehören auch Vertreter aus Industrie, Wirtschaft und Politik zum Stammpublikum, da sie hier die aktuellen Entwicklungen der Literatur mit verfolgen können.' (Ebd.). Es bleibt abzuwarten, welchen Einfluss das Internet noch auf diese Entwicklungen haben wird. Beispielsweise sind in der Filmkritik schon weitergehende Prozesse vollzogen worden. Auf dem bedeutendsten Onlineforum imdb, der Internet Movie Data Base, werden die Rezensionen des breiten Publikums neben den Metascore der Kritikergilde gestellt, sodass sich ein perspektivisch breiteres Spektrum ergibt. Die Stimme des nicht-professionellen Kritikers wird mit der des Profis gleichgestellt, wodurch die Forderung nach der Aufwertung des Amateur-Lesers umgesetzt wäre.

806 Literaturhäuser 2015.
} 
Autoren, das Lesen und das Leitmedium Buch. ${ }^{807}$ Der Buchpreis setzt sich demnach das als Intention, was Krausser in seinem Text+Kritik-Essay zu Kehlmann von der deutschen Kritik eingefordert hatte, um der deutschen Gegenwartsliteratur auf das laut ihm nächsthöhere Level zu verhelfen: die Weiterempfehlung an das Ausland. Der Literaturpreis zielt dezidiert auf den internationalen Markt. Der Preis beschränkt sich dabei nicht auf die bloße Weiterempfehlung von Autoren, sondern strebt danach, der Literatur ihren Platz in der modernen Gesellschaft zu sichern und ihre Zukunft als etabliertes und populäres Medium sicherzustellen.

Die Eventisierung der Literatur, wie sie durch die Literaturhäuser, den Literaturpreis, Lesereisen oder Poetikvorlesungen vorangetrieben wird, scheint der Literatur wieder vermehrt einen Platz im öffentlichen Diskurs zu sichern. Ein weiteres Forschungsfeld wird es sein, den Einfluss der Lesbarkeit auf mündliche Rezeptionsformen wie die sich zunehmender Beliebtheit erfreuenden Poetry Slams zu erschließen. Es wird zu fragen sein, ob das eine das andere oder beide sich gegenseitig bedingen. Weil der Lesbarkeitsbegriff in der klassischen Rhetorik gründet, dürfte bereits ein ausreichendes theoretisches Instrumentarium vorhanden sein, um sich dieser Problemstellung wissenschaftlich zu nähern.

Abschließend lässt sich konstatieren, dass das Eintreten von Wittstock, Politycki und Hielscher für das Programm einer 'Neuen Deutschen Lesbarkeit' in den 1990er Jahren sowie später das Wirken von Autoren wie Krausser, Kehlmann und Glavinic dazu geführt haben, dass Vertreter der jungen Generation wie Benedict Wells (Jahrgang 1984), die vom Einfluss der Lesbarkeit bewirkten literarischen Chancen voll und ohne Vorurteile seitens des Literaturbetriebs ausschöpfen können. Dies wird aus einem Beitrag ersichtlich, den Wells 2012 in der programmatisch 'Über das Erzählen' betitelten Ausgabe des $D u$ Magazins zum 60. Jubiläum des Diogenes Verlags veröffentlichte. Sein Essay 'Und, wie geht die Geschichte weiter? Sag schon!' setzt ein auf Lesbarkeit zielendes Literaturprogramm als selbstverständlich voraus. ${ }^{808}$

Wells setzt das folgende Szenario als Ausgangspunkt seiner Literatur: 'Ich glaube, die Urform der Erzähler sind Menschen, die am Lagerfeuer sitzen und sich Geschichten für ihre Zuhörer ausdenken. Fast alle grossen Romane basieren auf dieser klassischen

\footnotetext{
807 Buchpreis 2015.

808 Diogenes setzt in seiner gegenwärtigen Verlagsgeschichte dezidiert auf das auf Molière zurückgehende Motto, 'jede Art zu schreiben ist erlaubt - nur die langweilige nicht', und etabliert damit Unterhaltung als wichtigstes Publikationskriterium. Vgl. Diogenes 2015.
} 
Art des Erzählens' ${ }^{809}$ Wells beginnt mit einer mündlichen Form des Erzählens. Ähnlich wie Ernst Keil in der programmatischen Einführung seiner Zeitschrift Die Gartenlaube evoziert Wells einen idealen und angenehmen Rezeptionsort, der vor allem eine unmittelbare Begegnung zwischen Erzähler und Rezipienten ermöglicht. Es wird dezidiert für das Publikum erzählt und es kann daher davon ausgegangen werden, dass der Text für das Publikum zugänglich und wirksam gestaltet wird. ${ }^{810}$ Zudem steht das Adjektiv 'klassisch' für einen Rückgriff auf bewährte Traditionen. Diese liegen für Wells jedoch wie auch für die zuvor besprochenen Befürworter von Lesbarkeit nicht primär im deutschsprachigen, sondern im anglo-amerikanischen Raum:

\begin{abstract}
Romane, die keine Handlung und keine Geschichten haben, keine Entwicklung und keine Charaktere, denen ich bedingungslos bis ans Ende der Welt folgen würde; solche Romane habe ich fast immer weggelegt, selbst wenn sie grossartig geschrieben waren. Gerade mit der gedanklich oft überfrachteten, sperrigen deutschen Literatur konnte ich deshalb nie viel anfangen. [...] Was mir generell an ausländischen Romanen gefällt, ist die Leichtigkeit, mit der sie erzählt sind. So schultern sie auch schwierige Themen, ohne das Gewicht auf den Leser abzuwälzen. ${ }^{811}$
\end{abstract}

Wells betont die Wichtigkeit von Kategorien, die inhärent für den Lesbarkeitsbegriff sind. Er stellt wie Eco die Eminenz der Handlung heraus, die in Form der aristotelischen, stringent abgerundeten Handlungsführung auftritt. Außerdem ist die Identifikation mit den Figuren, wie auch die Romananalysen der vorhergehenden Kapitel gezeigt haben, ein zentrales Mittel, um die Leser für einen Text zu gewinnen. Stilistische Qualität tritt hier zurück und wird zu einem nebensächlichen Parameter im Vergleich zu Handlung und Figuren. Wells' Ablehnung der deutschsprachigen Literatur resultiert aus deren vermeintlicher Komplexität. Er betont deren Fokussierung auf den Intellekt, die ihm zufolge keinen emotionalen Zugang zu den Figuren und der Handlung ermöglicht. An der ausländischen Literatur lobt er hingegen, die auch von den Vertretern der Lesbarkeit angeführte Qualität, ein komplexes Sujet möglichst leicht zugänglich zu bearbeiten. Demnach disqualifiziert für Wells der Mangel an Lesbarkeit die deutsche Literatur, und deren aktive Inanspruchnahme wertet für ihn die Literatur aus dem

\footnotetext{
809 Wells 2012, S. 34.
}

810 An einer späteren Stelle sieht er zudem den Vorteil der Literatur gegenüber anderen Kunstformen darin, dass der Leser bei der Lektüre selbst Teil des kreativen Prozesses ist und das Werk letzten Endes erst durch das Lesen vervollständigt wird, was die Position des Lesers gegenüber dem Autor aufwertet; vgl. ebd., S. 36.

811 Ebd. 
Ausland auf. ${ }^{812}$ Er fordert in der Terminologie Franzens eine Einhaltung des Vertrags zwischen dem Autor und seinen Lesern.

Als Abschluss seines Essays stellt Wells einen Reifeprozess dar, den er als Autor nach wie vor vollziehe. Das Ziel dabei sei jedoch keine größere Komplexität. Er führt an, seinen Roman Fast genial (2011) 'bewusst vereinfacht' zu haben. Trotz des ethisch schwierigen Themas der Präimplantationsdiagnostik sollte der Roman 'so leicht wie möglich werden': 'Mit Fast genial wollte ich zurück ans Lagerfeuer und eine Geschichte erzählen, die man einfach gern liest. Gerade dieses Einfache, Flotte stellte sich jedoch als schwierig heraus und benötigte beim Überarbeiten am meisten Zeit. ${ }^{, 813}$ Wells richtet den Schreibprozesses direkt auf den Leser aus, für den er die Lektüre so angenehm und anregend wie möglich gestalten will. Das Erzählen mit dem Ziel des delectare gilt als ultimative Form, die es als Autor anzustreben gilt. Die schriftstellerische Kompetenz, die notwendig ist, um eine leichte und einfache Literatur zu erschaffen, muss Wells nicht erst, wie es das Programm der 'Neuen Deutschen Lesbarkeit' unternehmen musste, legitimieren. Er nimmt sie als gegebene anspruchsvolle Herausforderung an. Die in der deutschsprachigen Literatur von ambitionierten Autoren tendenziell privilegierte Komplexität und einen hohen ästhetischen Anspruch lehnt er zugunsten der Lesbarkeit ab, deren nachhaltiger poetologischer Einfluss es ihm ermöglicht, eine Literatur zu verfassen, die explizit an ein breites Publikum gerichtet ist. Es handelt sich um eine Auffassung von Literatur und literarischer Qualität, die einen scheinbar simplen, jedoch lange umkämpften und schwer zu bewerkstelligenden, aus der klassischen Rhetorik stammenden Grundsatz verfolgt: nämlich, wie Wells es ausdrückt, so 'einfach, klar und präzise' wie möglich zu sein. ${ }^{814}$ Lesbarkeit ist demnach für eine neue Generation junger Autoren zum Leitbild geworden.

\footnotetext{
812 Als Beispiele nennt er Kazuo Ishiguro, Ernest Hemingway, John Irving und Scott Fitzgerald (Ebd.).

813 Ebd., S. 35.

814 Vgl. ebd., S. 36.
} 


\section{Bibliographie}

\section{Primärquellen}

\section{a. Ulrike Draesner}

Draesner, Ulrike 2007: Zauber im Zoo. Vier Reden von Herkunft und Literatur. Göttingen: Wallstein (Vorlesungen gehalten 2006).

Draesner, Ulrike 2014 a: Der Siebte Sprung. In: http://www.dersiebte-sprung.de (Zugang 22. 05. 2015).

Draesner, Ulrike 2014 b: Affen. In: http://www.der-siebte-sprung.de/ category/affen/> (Zugang 22. 05. 2015).

Draesner, Ulrike 2014 c: Deutsche Quellen. In: http://www.der-siebtesprung.de/deutsche-quellen/ (Zugang 22. 05. 2015).

Draesner, Ulrike 2014 d: Essay 2. In: http://www.der-siebtesprung.de/rendez-vous-2-7tersprung/ (Zugang 22. 05. 2015).

Draesner, Ulrike 2014 e: Lexikon. In: http://www.der-siebtesprung.de/category/lexikon-der-reisenden-woerter/ (Zugang 22. 05. 2015).

Draesner, Ulrike 2014 f: Polnische Quellen. In: http://www.der-siebtesprung.de/polnische-quellen (Zugang 22. 05. 2015).

Draesner, Ulrike 2014 g: Postmemory. In: http://www.der-siebte-sprung.de/ postmemory/ (Zugang 22. 05. 2015).

Draesner, Ulrike 2014 h: Romanwege. In: http://www.der-siebte-sprung.de/ romanwege/ (Zugang 22. 05. 2015).

Draesner, Ulrike 2014 i: Schweinfurthii. In: http://www.der-siebte-sprung.de/ pan-troglodytes-schweinfurthii/ (Zugang 22. 05. 2015).

Draesner, Ulrike 2014 j: Selbst Erzählen. In: http://www.der-siebtesprung.de/selbst-erzaehlen/ (Zugang 22. 05. 2015).

Draesner, Ulrike 2014 k: Sieben Sprünge vom Rand der Welt. München: Luchterhand. 


\section{b. Thomas Glavinic}

Glavinic, Thomas 1999 a: Wie möchtest du dein Ei? In: Spectrum, 27. März, S. III.

Glavinic, Thomas 1999 b: Ein Auftrag von der Fee. In: Die Presse, 14. August, S. VI.

Glavinic, Thomas 2010: Das bin doch ich. München: Deutscher Taschenbuch Verlag (Erstveröffentlichung: München: Hanser 2007).

Glavinic, Thomas 2011: Das Leben der Wünsche. München: Deutscher Taschenbuch Verlag (Erstveröffentlichung: München: Hanser 2009).

Glavinic, Thomas 2013 a: Das größere Wunder. München: Hanser.

Glavinic, Thomas 2013 b: Das größere Wunder. Thomas Glavinics großes Buch der Liebe. In: http://www.thomas-glavinic.de/2013/05/das-groessere-wunder/ (Zugang 19. 05. 2015).

Glavinic, Thomas 2013 c: Lisa. München: Deutscher Taschenbuch Verlag (Erstveröffentlichung: München: Hanser 2011).

Glavinic, Thomas 2013 d: Die Vermessung des Daniel Kehlmann. In: Wiener Online, 18. April. http://wiener-online.at/home/menschen/glavinic/ 1390647/Daniel-Kehlmann (Zugang 28. 09. 2014).

Glavinic, Thomas 2014 a: Meine Schreibmaschine und ich. München: Hanser (Vorlesungen gehalten 2014).

Glavinic, Thomas 2014 b: Der Roman. In: http://www.thomasglavinic.de/2009/06/der-roman/ (Zugang 13. 11. 2014. Erstveröffentlichung: Akzente 4/2008).

\section{c. Felicitas Hoppe}

Hoppe, Felicitas 2009 a: Iwein Löwenritter. Nach einem Roman von Hartmann von Aue. Frankfurt/M.: Fischer (Erstveröffentlichung 2008).

Hoppe, Felicitas 2009 b: Sieben Schätze. Augsburger Poetikvorlesungen. Frankfurt/M.: Fischer (Vorlesungen gehalten 2008).

Hoppe, Felicitas 2012: Hoppe. Frankfurt/M.: Fischer. 


\section{d. Daniel Kehlmann}

Kehlmann, Daniel 2006: Die Hölle sind nicht die anderen. In: Der Spiegel. 31. Juli, S. 128f.

Kehlmann, Daniel 2009: Ruhm. Ein Roman in neun Geschichten. Reinbek bei Hamburg: Rowohlt.

Kehlmann, Daniel 2010 a: 'Mein Ich ist ein Projekt'. Helmut Kraussers Tagebücher. In: Tom Kindt (Hg.): Helmut Krausser. München: Text + Kritik, Heft 187, S. 44-48.

Kehlmann, Daniel 2010 b: Die Vermessung der Welt. Reinbek bei Hamburg: Rowohlt (Erstveröffentlichung 2005).

Kehlmann, Daniel 2010 c: Wo ist Carlos Montúfar? Über Bücher. Reinbek bei Hamburg: Rowohlt (Erstveröffentlichung 2005).

Kehlmann, Daniel 2011 a: Lob. Über Literatur. Reinbek bei Hamburg: Rowohlt (Erstveröffentlichung 2010).

Kehlmann, Daniel 2011 b: Die Katastrophe des Glücks. In Kehlmann 2011 a, S. 169-178 (Erstveröffentlichung: Die Welt, 10. 11. 2007).

Kehlmann, Daniel 2011 c: Diese sehr ernsten Scherze. In: Kehlmann 2011 a, S. 125-168 (Vorlesungen gehalten 2006. Erstveröffentlichung: Göttingen: Wallstein 2007).

Kehlmann, Daniel 2013: F. Reinbek bei Hamburg: Rowohlt.

Kehlmann, Daniel 2015: Kommt, Geister. Reinbek bei Hamburg: Rowohlt (Vorlesungen gehalten 2014).

Kehlmann, Daniel, und Kleinschmidt, Sebastian 2010: Requiem für einen Hund. Ein Gespräch. Reinbek bei Hamburg: Rowohlt.

\section{e. Helmut Krausser}

Krausser, Helmut 1995: Mai/Juni. Reinbek bei Hamburg: Rowohlt (Erstveröffentlichung: München: belleville 1993-1994).

Krausser, Helmut 1997: Juli/August/September. Reinbek bei Hamburg: Rowohlt (Erstveröffentlichung: München: belleville 1996-1997). 
Krausser, Helmut 2000: Oktober/November/Dezember. Reinbek bei Hamburg: Rowohlt (Erstveröffentlichung: München: belleville 1999-2000).

Krausser, Helmut 2003: Januar/Februar. Reinbek bei Hamburg: Rowohlt (Erstveröffentlichung: München: belleville 2001-2002).

Krausser, Helmut 2006: März/April. Reinbek bei Hamburg: Rowohlt (Erstveröffentlichung: München: belleville 2003-2004).

Krausser, Helmut 2008: Ich und Kehlmann. Und 'Mahlers Zeit'. In: Heinz Ludwig Arnold (Hg.): Text + Kritik, Heft 177, S. 54-57.

Krausser, Helmut 2012: Nicht ganz schlechte Menschen. Köln: DuMont.

Krausser, Helmut 2014: Deutschlandreisen. Köln: DuMont.

\section{Andere Quellen und Forschungsliteratur}

Adam, Christian 2010: Lesen unter Hitler. Autoren, Bestseller, Leser im Dritten Reich. Berlin: Galiani.

Alber, Jan, und Fludernik, Monika (Hg.) 2010: Postclassical Narratology. Approaches and Analyses. Columbus: The Ohio State University Press.

Allen, Grant 1897: Some New Books. The Fortnightly Review. Juli, S. 144-154.

Armand D'Angour 2011, The Greeks and the New Cambridge: Cambridge University Press.

Anonymus 2007 a. Beste Erzähltradition. Frankfurter Allgemeine Zeitung, 30. März, S. 57.

Anonymus 2007 b. Man bleibt als Erzähler immer ein Dilettant. Der Autor Daniel Kehlmann über Musterschüler und Mittelmaß. Stuttgarter Zeitung, 03. November, S. 37.

Anonymus 2007 c. Ohne jeden Komplex. Frankfurter Allgemeine Zeitung, 30. März, S. 57.

Anonymus 2007 d. 174 Zentimeter. Frankfurter Allgemeine Zeitung, 30. März, S. 57.

Anonymus 2013. Das geht nicht spurlos an einem vorüber. Profil, 07. Januar, S. 65.

Anz, Thomas (Hg.) 1995: Es geht nicht um Christa Wolf. Der Literaturstreit im vereinten Deutschland. Frankfurt/M.: S. Fischer (Erstveröffentlichung 1991). 
Aristoteles 1994: Poetik. Gr./Dt. Übers. u. hg. v. Manfred Fuhrmann. Stuttgart: Reclam.

Aristoteles 2007: Rhetorik. Gr./Dt. Übers. u. hg. v. Gernot Krapinger. Stuttgart: Reclam.

Arnold, Heinz Ludwig (Hg.) 1980: Die Gruppe 47. Eine kritischer Grundriss. München: Text + Kritik.

Arnold, Heinz Ludwig 2004: Die Gruppe 47. Reinbek bei Hamburg: Rowohlt.

Auerbach, Erich 1977: Mimesis. Dargestellte Wirklichkeit in der abendländischen Literatur. 6. Aufl. Bern: Francke (Erstveröffentlichung 1946).

Auffermann, Verena 1993: Was in den Sternen steht. Süddeutsche Zeitung, 13./14. März, S. IV.

Aust, Hugo 2000: Lesen. In: Weimar 1997-2003, Bd. 2, S. 406-410.

Baron, Ulrich 1996: Der talentierte Herr Johanser. Rheinischer Merkur, 01. März, S. 21.

Bartel, Jörg 1993: Ein magischer Musik-Roman. Neue Ruhr Zeitung, 18. September, S. 56.

Barthes, Roland 1973: Le plaisir du texte. Paris: Éditions du Seuil.

Becher, Johannes R. 1962: Über Literatur und Kunst. Berlin: Aufbau.

Becker, Sabina 2000: Neue Sachlichkeit. Weimar: Böhlau.

Becker, Tobias 2013: Drogen in Hamburg, Massage in São Paulo. Spiegel Online, 26. August, In: http://www.spiegel.de/kultur/literatur/das-groessere-wunder-roman-vonthomas-glavinic-a-918300.html (Zugang 25. 03. 2014).

Beyer, Susanne 2014: Irgendetwas ist da mit euch. Der Spiegel, 24. November, S. 120-122.

Biendarra, Anke S. 2012: Germans Going Global. Contemporary Literature and Cultural Globalization. Berlin: De Gruyter.

Böhn, Andreas 2003: Wirkungsästhetik. In: Weimar 1997-2003, Bd. 3, S. 851-854. 
Böll, Heinrich 1977: Frankfurter Vorlesungen. München: Deutscher Taschenbuch Verlag (Vorlesungen gehalten 1964. Erstdruck: Köln: Kiepenheuer \& Witsch 1966).

Böll, Heinrich 1998-: Werke. Hg v. Árpad Bernáth und Annamária Gyurácz. 27 Bde. Köln: Kiepenheuer \& Witsch.

Biller, Maxim 1992: Dekadenzen. Tempo, Juni, S. 154.

Biller, Maxim 1998: Soviel Sinnlichkeit wie der Stadtplan von Kiel.

Warum die neue deutsche Literatur nichts so nötig hat wie den Realismus. Ein Grundsatzprogramm. In: Andrea Köhler und Rainer Moritz (Hg.): Maulhelden und Königskinder. Zur Debatte über die deutschsprachige Gegenwartsliteratur. Leipzig: Reclam, S. 62-71 (Erstveröffentlichung: Weltwoche, 15. 07. 1991).

Borchert, Wolfgang 1949: Das Gesamtwerk. Reinbek bei Hamburg: Rowohlt.

Braun, Rebecca 2013: Daniel Kehlmann, 'Die Vermessung der Welt'. Measuring Celebrity through the Ages. In: Marven, Lyn, und Taberner, Stuart (Hg.): Emerging German-Language Novelists of the Twenty-First Century. New York: Camden House, S. 75-88 (Erstveröffentlichung 2011).

Buchpreis 2015: Über den Preis. In: http://www.deutscher-buchpreis.de/der-preis/ (Zugang 19. 06. 2015).

Burger, Jörg 1997: Helden der Popkultur. ZEITmagazin, 19. September, S. 5.

Catani, Stephanie 2008: Formen und Funktionen des Witzes, der Satire und der Ironie in 'Die Vermessung der Welt'. In: Nickel 2008, S. 198-215.

Cicero, Marcus Tullius 2001: De oratore. Lat./dt. Übers. u. hg. v. Harald Merklin. Stuttgart: Reclam.

Cicero, Marcus Tullius 2004: Orator. Lat./dt. Übers. u. hg. v. Harald Merklin. Stuttgart: Reclam.

Claudius, Eduard 1984: Menschen an unserer Seite. Stuttgart: Neuer Weg (Erstveröffentlichung: Halle: Mitteldeutscher Verlag 1951).

David, Thomas 2009: Unser Selbst ist immer gespalten. Du, März, S. 29-32.

Deiritz, Karl, und Krauss, Hannes (Hg.) 1991: Der deutsch-deutsche Literaturstreit oder 'Freunde, es spricht sich schlecht mit gebundener Zunge'. Hamburg: Luchterhand.

De Jong, Irene 2014: Narratology \& Classics. A Practical Guide. Oxford: Oxford University Press. 
Dillane, Fionnuala 2013: Before George Eliot. Marian Evans and the Periodical Press. Cambridge: Cambridge University Press.

Diogenes 2015: Geschichte. In: http://www.diogenes.ch/leser/verlag/geschichte (Zugang 24. 06. 2015).

Drosdowski, Günther (Hg.) 2013: Etymologie. Herkunftswörterbuch der deutschen Sprache. Mannheim: Duden 2013.

Duden 2016: Lesbar. In: http://www.duden.de/rechtschreibung/lesbar (Zugang 07. 02. 2016).

Eco, Umberto 1986: Nachschrift zum Namen der Rose. Übers. Burkhart Kroeber. München: Deutscher Taschenbuch Verlag (ital. Orig.: Postille al Nome della rosa. Mailand: Alfabeta 1983).

Emmerich, Wolfgang 1989: Kleine Literaturgeschichte der DDR. Frankfurt/M.: Luchterhand (Erstveröffentlichung: Darmstadt: Luchterhand 1981).

Ernst, Thomas 2001: Popliteratur. Hamburg: Rotbuch.

Fähnders, Walter 1998: Avantgarde und Moderne. 1890-1933. Stuttgart: Metzler.

Fasthuber, Sebastian 2014: Seit 15 Jahren polarisiert Helmut Krausser mit seinen Büchern Leser und Kritiker. In: http://www.bernd-pleis.de/interview_link.asp (Zugang 30. 09. 2014).

Fauser, Markus 2003: Unterhaltung (1). In: Weimar 1997-2003, Bd. 3, S. 728-730.

Fenzl, Christa 1993: Und du hast mich wirklich gesucht? Main-Echo, 05. Februar, S. 26.

Fiedler, Leslie A. 1971: Cross the Border-Close the Gap. In: Ders. (Hg.): The Collected Essays of Leslie Fiedler. 2 Bde. New York: Stein and Day 1971, Bd. 2, S. 461-485 (Erstveröffentlichung: Playboy, Dezember 1969).

Finlay, Frank 2007: Literary Debates and the Literary Market since Unification. In: Taberner 2007, S. 21-38.

Fludernik, Monika 2003: The Diachronization of Narratology. In: Narrative, 11:3, S. 331-348.

Fludernik, Monika 2009: An introduction to narratology. Abingdon: Routledge.

Fludernik, Monika 2011: The Category of 'Person' in Fiction. 'You' and 'We' NarrativeMultiplicity and Indeterminacy of Reference. In: Olson 2011, S. 101-141. 
Fludernik, Monika, und Olson, Greta 2011: Introduction. In: Olson 2011, S. 1-33.

Fontane, Theodor 1962-1997: Sämtliche Werke. Hg. v. Walter Keitel und Helmuth Nürnberger. 22 Bde. München: Hanser.

Fontane, Theodor 1968-1971: Briefe. Hg. v. Kurt Schreinert. 4 Bde. Berlin: Propyläen.

Fontane, Theodor 1980: Briefe. Hg. v. Nationale Forschungs- und Gedenkstätten der klassischen deutschen Literatur in Weimar. 2 Bde. Aufbau: Berlin.

Fontane, Theodor 1995-2012: Tagebücher und Reisetagebücher. 3 Bde. 2. Aufl. Hg. v. Gotthard Erler und Therese Erler. Berlin: Aufbau.

Franzen, Jonathan 2002: Mr. Difficult. In: Shakespeare Collection. Gale. University of Oxford (Zugang 13. 12. 2015. Erstveröffentlichung: The New Yorker, 30. 09. 2002)

Franzen, Jonathan 2010: How to be alone. London: Fourth Estate (Erstveröffentlichung: New York: Farrar, Straus and Giroux 2002).

Franzen, Jonathan, und Haslett, Adam 2010: Are we feeling better now? Fiktion und Autobiographie. Künzelsau: Swiridoff (Vorlesungen gehalten 2009).

Franzen, Günter 1996: Ein Germanist sieht rot. Die Zeit, 29. März, S. 2.

Friedrich, Hans-Edwin 2000: Kitsch. In: Weimar 1997-2003, Bd. 2, S. 263-266.

Geißler, Cornelia 2012: Andere trinken Schnaps. Frankfurter Rundschau Online, 17. Dezember. In: http://www.fr-online.de/literatur/felicitas-hoppe-andere-trinkenschnaps, 1472266,21136198.html (Zugang 15. 01. 2016).

Genette, Gérard 1987: Seuils. Paris: Éditions du Seuil.

Goethe, Johann Wolfgang 1985-1999: Sämtliche Werke, Briefe, Tagebücher und Gespräche. Hg. v. Friedmar Apel u.a. 40 Bde. Frankfurt/M.: Deutscher Klassiker Verlag.

Gösweiner, Friederike 2013: Happy End am Everest. Die Presse, 24. August, S. VII.

Gollner, Helmut 2005: Die Wahrheit lügen. Die Renaissance des Erzählens in der jungen österreichischen Literatur. Innsbruck: Studienverlag.

Greiner, Ulrich 1991: Die deutsche Gesinnungsästhetik. In: Deiritz/Kraus 1991, S. 139-145 (Erstveröffentlichung: Die Zeit, 02. 11. 1990)

Greiner, Ulrich 2007: Ulrich Greiners Leseverführer. Eine Gebrauchsanweisung zum Lesen schöner Literatur. München: Deutscher Taschenbuch Verlag (Erstveröffentlichung: München: Beck 2005). 
Grimm, Jakob, und Grimm, Wilhelm 1984: Deutsches Wörterbuch. Leipzig: 1854-1971. Repr. Nachdr.: 33 Bde. München: Deutscher Taschenbuch Verlag.

Großmann 2004. Großmann, Patrick 2004: Den Hang zur ständigen Verfeinerung überlasse ich anderen. 02. Mai. In: http://www.helmut-krausser.de/ (Zugang 01. 09. 2014).

Gutzkow, Karl 2001: Werke und Briefe. Kommentierte digitale Gesamtausgabe. Hg. v. Gert Vonhoff und Martina Lauster. Münster: Oktober. In: http://projects.exeter.ac.uk/ gutzkow/Gutzneu/gesamtausgabe/index.htm (Zugang 07. 01. 2014).

Haferkorn, Hans Jürgen 1963: Der freie Schriftsteller. Eine literatur-soziologische Studie über seine Entstehung und Lage in Deutschland zwischen 1750 und 1800. In: Börsenblatt für den Deutschen Buchhandel. Frankfurter Ausgabe 19, S. 125-219.

Hage, Volker 1999: Ganz schön abgedreht. Der Spiegel, 22. März, S. 244-246.

Hagestedt, Lutz 1992: Babykiller Herodes füllt das Sommerloch aus. Süddeutsche Zeitung, 07. Mai, S. IV.

Hagestedt, Lutz 1993: Orpheus' Leier hat neue Saiten bekommen. Badische Zeitung, 18. April, S. 15.

Hall, Katharina 2006: The Author, the Novel, the Reader and the Perils of 'Neue Lesbarkeit': A Comparative Analysis of Bernhard Schlink's 'Selbs Justiz' and 'Der Vorleser'. In: German Life and Letters, 59:3, S. 446-467.

Halliwell, Stephen 2013: Diegesis - Mimesis. In: Hühn, Peter et al. (Hg.): The living handbook of narratology. Hamburg: Hamburg University. http://www.lhn.unihamburg.de/article/diegesis---mimesis (Zugang 01. 02. 2016).

Halverson, Rachel 2010: The New German Lesbarkeit in Action. Narrative and Context in Martina Hefter's Zurück auf Los. In: Symposium, 64:2, S. 73-89.

Hamouda, Fayçal (Hg.) 2005: Der Leipziger Verleger Ernst Keil und seine 'Gartenlaube'. Leipzig: Edition Marlitt.

Harding, Luke 2006: Unlikely bestseller heralds the return of lightness and humour to German literature. The Guardian, 19. Juli, S. 21.

Hartmann von Aue 2012: Iwein. Mhd./nhd. Übers. u. hg. v. Rüdiger Krohn u. hg. v. Mireille Schnyder. Stuttgart: Reclam.

Hegel, Georg Wilhelm 1986: Werke. Auf der Grundlage der 'Werke' von 1832-1845 neu edierte Ausgabe. Hg. v. Eva Moldenhauer und Karl Markus Michel. 20 Bde. Frankfurt/M.: Suhrkamp.

Heinzelmann, Herbert 1993: Stimme Gottes. Nürnberger Zeitung, 28./29. August, S. 10. 
Herles, Wolfang 2013 a: Das blaue Sofa. ZDF. 13. September.

Herles, Wolfang 2013 b: Das blaue Sofa. ZDF. 25. Oktober.

Herman, David 1997: Scripts, Sequences, and Stories. Elements of a Postclassical Narratology. In: Publications of the Modern Languages Association of America. 12:5, S. 1046-1059.

Herman, David (Hg.) 1999: Narratologies. New Perspectives on Narrative Analysis. Columbus: The Ohio State University Press.

Herman, David (Hg.) 2007 a: The Cambridge Companion to Narrative. Cambridge: Cambridge University Press.

Herman, David 2007 b: Introduction. In: Herman 2007 a, S. 3-21.

Herzog, Reinhart, und Koselleck, Reinhart (Hg.) 1987: Epochenschwelle und Epochenbewusstsein. München: Fink.

Hielscher, Martin (Hg.) 1996: Wenn der Kater kommt. Neues Erzählen - 38 deutschsprachige Autorinnen und Autoren. Köln: Kiepenheuer \& Witsch.

Hielscher, Martin 2001: Geschichte und Kritik. Die neue Lesbarkeit und ihre Notwendigkeit. In: Zeitschrift für Literaturwissenschaft und Linguistik 31:124, S. 65-71.

Hierländer, Jeannine, und Stern, Nicole 2014: Darf man über Reiche nicht schreiben? Die Presse, 03. Februar, S. 14.

Höppner, Stefan, und Kreienbrock, Jörg (Hg.) 2015: Die amerikanischen Götter. Transatlantische Prozesse in der deutschsprachigen Literatur und Popkultur seit 1945. Berlin: De Gruyter.

Horatius, Quintus Flaccus 2011: Ars Poetica. Lat./dt. Übers. u. hg. v. Eckart Schäfer. Stuttgart: Reclam.

Horkheimer, Max, und Adorno, Theodor W. 2000: Dialektik der Aufklärung. Philosophische Fragmente. Frankfurt/M.: Fischer (Erstveröffentlichung: New York: Social Studies Association 1944).

Hugendick, David 2013: Ein neuer Mensch. Die Zeit, 12. September, S. 52.

Hunold, Christian Friedrich 1973: Satyrischer Roman. Hg. v. Hans Wagener. Bern: Lang (Erstveröffentlichung 1706).

Hunold, Christian Friedrich 1988 a: Die Verliebte und Galante Welt. Hg. v. Hans Wagener. Bern: Lang (Erstveröffentlichung 1707). 
Hunold, Christian Friedrich 1988 b: Der Verliebten und Galanten Welt Anderer Theil. In: Hunold 1988 a (Mit Seite 1 beginnend an den ersten Teil angeschlossen).

Hunold, Christian Friedrich (Hg.) 1991: Auserlesene und teils noch nie gedruckte Gedichte unterschiedener berühmten und geschickten Männer. Hildesheim: Olms (Erstveröffentlichung 1718).

Jäger, Georg 1997: Avantgarde. In: Weimar 1997-2003, Bd. 1, S. 183-187.

Johnson, Samuel 2006: Lives of the Most Eminent English Poets Volume 4. With Critical Observations of their Works. Oxford: Clarendon (Erstveröffentlichung 1781).

Kästner, Erich 1928: Indirekte Lyrik. In: Das Deutsche Buch 5/6 (Mai/Juni), S. 143-145.

Kästner, Erich 1959: Gesammelte Schriften. 6 Bde. Köln: Kiepenheuer \& Witsch.

Kaiser, Georg 1971-1972: Werke. Hg. v. Walther Huder. 6 Bde. Frankfurt/M.: Ullstein, Propyläen.

Kaminski, Nicola 2001: Männerliebe contra weibliche Autorschaft? Geteilte Spiele im Iwein Hartmanns von Aue. In: Oxford German Studies, 30:1, S. 26-51.

Kant, Immanuel 1908: Kritik der Urteilskraft. Hg. v. Wilhelm Windelband. Berlin: Reimer (Gesammelte Schriften. Hg. v. d. Königlich Preußischen Akademie der Wissenschaften. 1900-, Bd. 5), S. 165-488 (Erstveröffentlichung 1790).

Keil, Ernst 1853: An unsere Freunde und Leser! In: Die Gartenlaube, 01. Januar, S. 1.

Keil, Ernst 2005: Brief an eine Gläubige. In: Hamouda 2005, S. 147-153

(Erstveröffentlichung: Die Gartenlaube 1866).

Keller, Gottfried 1950: Gesammelte Briefe. Hg. v. Carl Helbling. 4 Bde. Bern: Bentell.

Keller, Andreas 2008: Frühe Neuzeit. Das rhetorische Zeitalter. Berlin: Akademie Verlag.

Kienzle, Michael 1975: Der Erfolgsroman. Zur Kritik seiner poetischen Ökonomie bei Gustav Freytag und Eugenie Marlitt. Stuttgart: Metzler.

Kiesel, Helmuth, und Münch, Paul 1977: Gesellschaft und Literatur im 18. Jahrhundert. Voraussetzungen und Entstehung des literarischen Markts in Deutschland. München: Beck (Beck'sche Elementarbücher).

Klauhs, Harald 2009: Haben Sie nichts erlebt, Herr Kehlmann? Die Presse, 22. März, S. $62 \mathrm{f}$. 
Kleinschmidt, Erich 2003 a: Prosa. In: Weimar 1997-2003, Bd. 3, S. 168-172.

Kleinschmidt, Erich 2003 b: Roman. In: Weimar 1997-2003, Bd. 3, S. 317-322.

Koch, Lennart 2001: Ästhetik der Moral bei Christa Wolf und Monika Maron. Der Literaturstreit von der Wende bis zum Ende der neunziger Jahre. Frankfurt/M.: Lang.

Kohl, Katrin 2007 a: Festival, Performance, Wettstreit. Deutsche Gegenwartsliteratur als Ereignis. In: Nicholas Saul und Ricarda Schmidt (Hg.): Literarische Wertung und Kanonbildung. Würzburg: Königshausen \& Neumann, S. 173-190.

Kohl, Katrin 2007 b: Poetologische Metaphern. Formen und Funktionen der deutschen Literatur. Berlin: De Gruyter.

Kohl, Katrin 2012: E. Marlitt's Bestselling Novels. In: Charlotte Woodford und Benedict Schofield (Hg.): The German Bestseller in the Late Nineteenth Century. Rochester: Camden House, S. 183-205.

Koselleck, Reinhart 1973: Kritik und Krise. Eine Studie zur Pathogenese der bürgerlichen Welt. Frankfurt/M.: Suhrkamp (Erstveröffentlichung Freiburg: Alber 1959).

Kraft, Thomas (Hg.) 2000: Aufgerissen. Zur Literatur der 90er. München: Piper.

Kraus, Karl 1910: Stil. In: Der Sturm, 1:4, S. $26 f$.

Krause, Tilman 1996: Wühlen im Wahn. Tagesspiegel, 22. Februar, S. 21.

Kröll, Friedhelm 1979: Gruppe 47. Stuttgart: Metzler.

Krumbholz, Martin 1997: Thanatos light. Freitag, 10. Oktober, S. X.

Kümmerling-Meibauer, Bettina 2000: Kinder- und Jugendliteratur. In: Weimar 1997-2003, Bd. 2, S. 254-258.

Kuhn, Thomas S. 1996: The Structure of Scientific Revolutions. Chicago: University of Chicago Press (Erstveröffentlichung: 1962).

Kurz, Rudolf 1910: Programmatisches. In: Der Sturm, 1:1, S. 2f.

Lee, Hermoine 2009: Biography. A Very Short Introduction. Oxford: Oxford University Press.

Lessing, Gotthold Ephraim 1985: Werke. Hg. v. Wilfried Barner et al. 12 Bde. Frankfurt/M.: Deutscher Klassiker Verlag. 
Leurs, Rainer 2014: Fußball-Sprachgeschichte. Wie Deutschland das 'Schland' entdeckte. Spiegel Online, 04. Juli. In: http://www.spiegel.de/panorama/schland-wiestefan-raab-das-wort-schlaaand-in-umlauf-brachte-a-978845.html (Zugang 25. 05. 2015).

Link, Heiner 2014: Interview: In: http://www.bernd-pleis.de/interview_link.asp (Zugang 30. 09. 2014).

Literaturhäuser 2015: Das Netzwerk der Literaturhäuser. In: http:// www.literaturhaus.net/netzwerk/index.htm (Zugang 23. 06. 2015).

Löschnigg, Martin 2010: Postclassical Narratology and the Theory of Autobiography. In: Alber/Fludernik 2010, S. 255-274.

Lovenberg, Felicitas von 2006: Ich wollte schreiben wie ein verrücktgewordener Historiker. Frankfurter Allgemeine Zeitung, 09. Februar, S. 41.

Lovenberg, Felicitas von 2008: In wie vielen Welten schreiben Sie, Herr Kehlmann? Frankfurter Allgemeine Zeitung, 27. Dezember, S. Z6.

Lützeler, Paul Michael 1997: Klio oder Kalliope? Literatur und Geschichte. Sondierung, Analyse, Interpretation. Berlin: Schmidt.

Lux, Joseph August 1910: Kunst und Ethik. In: Der Sturm, 1:2, S. 13f.

Maar, Michael 2008: Qualität allein reicht nicht. Die Weltwoche, 15. Mai, S. 60-67.

Magenau, Jörg 2013: Der Geschmack der Höhenluft. Süddeutsche Zeitung, 27. August, S. 14.

Mangold, Ijoma 2005: Da lacht der Preuße, und der Franzose staunt. Süddeutsche Zeitung, 24. September, S. 16.

Mann, Thomas 1949: Ansprache im Goethejahr 1949. Weimar: Thüringer Volksverlag.

Mann, Thomas 2002-2011: Briefe. Hg. v. Thomas Sprecher, Hans R. Vaget und Cornelia Bernini. 3 Bde. Frankfurt/M.: Fischer.

Mann, Thomas 2013: Der Zauberberg. Frankfurt/M.: Fischer (Erstveröffentlichung: 1924).

Marlitt, Eugenie 1870: Reichsgräfin Gisela. Leipzig: Keil.

Mayer, Andrea, und Austilat, Andreas 2008: 'Ich mache mich jetzt sicher unbeliebt'. Daniel Kehlmann findet Österreichs Nationaldichter Thomas Bernhard verlogen. Der Tagesspiegel, 01. August, S. Ö1.

Meller, Marius 2006: Hamlet trifft Pythagoras. Der Tagesspiegel, 15. März, S.27. 
Meyer, Herbert 1958: Der Publizist, in Schiller 1943-2012, Bd. 20, S. 335-342.

Moritz, Rainer 2009: Der Avantgardist in der Medienfalle. Seltener Fall. Daniel Kehlmann ist der Liebling des Publikums und zugleich der Literaturkritik. Stuttgarter Zeitung, 21. Februar, S. 42.

Neukirch, Benjamin 1961: Benjamin Neukirchers Anthologie Herrn von Hoffmannswaldau und andrer Deutschen auserlesener und bißher ungedruckter Gedicht erster theil. Hg. v. Angelo D. de Capua u. Ernst A. Philippson. Tübingen: Niemeyer (Erstveröffentlichung 1695).

Neunzig, Hans A. (Hg.) 1979: Hans Werner Richter und die Gruppe 47. München: Nymphenburger.

Nickel, Gunther (Hg.) 2008: Daniel Kehlmanns 'Die Vermessung der Welt'. Materialien, Dokumente, Interpretationen. Reinbeck bei Hamburg: Rowohlt.

Norrick, Neal R. 2007: Conversational storytelling. In: Herman 2007 a, S. 127-141.

Nüchtern, Klaus 2007: Das bin doch nicht ich. Falter, 24.-30. August, S. 59.

Nüchtern, Klaus 2009: Beim Nasenbohren sieht jeder hin! Falter, 14. Januar, S. 24-27.

Olson, Greta (Hg.) 2011: Current Trends in Narratology. Berlin: De Gruyter.

Oltermann, Philip 2008: I and I. London Review of Books, 14. August, S. $36 f$.

Onno-Eisenbart, Carl 1910: Literarische Ausländerei? In: Der Sturm, 1:25, S. 200.

Opp, Annemarie 2016: 'The Game Is Never Over'. Das Spiel transmedialen Erzählens in Sherlock. In: Jonas Nesselhauf und Markus Schleich (Hg.): Das andere Fernsehen?Eine Bestandsaufnahme des 'Quality Television'. Bielefeld: transcript, S. 141-158.

Ottmers, Clemens 2007: Rhetorik. Stuttgart: Metzler.

Paul, Georgina 2009: Perspectives on Gender in Post-1945 German Literature. New York: Camden House.

Paterno, Wolfgang 2006: Am liebsten würde ich das Buch in die Ecke schmeißen. Profil, 02. Juni, S. 135-139.

Phelan, James 2007: Rhetoric/ethics. In: Herman 2007 a, S. 203-216. 
Pluto-Prondzinski, Thomas von 2014: 'Mit der Sprache Seiltanzen, das Gehört ins Varieté'. Zur Neusachlichkeit in Erich Kästners Texten von 1927 bis 1955'. In: Sebastian Schmierer und Johan Zonneveld (Hg.): Kästner im Spiegel. Beiträge der Forschung zum 40. Todestag. Marburg: Tectum, S. 229-254.

Politycki, Matthias 1998 a: Die Farbe der Vokale. Von der Literatur, den 78ern und dem Gequake satter Frösche. München: Luchterhand.

Politycki, Matthias 1998 b: Abschied von der Literatur. Was wir schon viel zu lange lesen müssen - ein fast frei erfundenes Gespräch. In: Politycki 1998 a, S. 45-50 (Erstveröffentlichung: Süddeutsche Zeitung, 12. 06. 1996).

Politycki, Matthias 1998 c: Gemischte Gefühle beim Verzehr von Hummerschwänzen. Über das Vergnügen an poetischen Gegenständen. In: Politycki 1998 a, S. 75-80 (Erstveröffentlichung: In: Günther Busch u. Uwe Wittstock (Hg.): Neue Rundschau, 2 [1995]).

Politycki, Matthias 1998 d: Kalbfleisch mit Reis! Die literarische Ästhetik der 78erGeneration. In: Politycki 1998 a, S. 23-44 (Vorlesung gehalten 1997. Erstveröffentlichung In: Norbert Wehr (Hg.): Schreibheft. Zeitschrift für Literatur, 50 [1997]).

Politycki, Matthias 1998 e: Literatur muss sein wie Rockmusik. In: Politycki 1998 a, S. 65-74 (Erstveröffentlichung: Frankfurter Rundschau, 07. 10. 1995).

Politycki, Matthias 1998 f: Neue Äusserlichkeit. In: Politycki 1998 a, S. 5-10.

Politycki, Matthias 1998 g: Die 78er und der Untergang des Hauses Usher. Was dahintersteckt, wenn sich Kritiker und Lektoren um die neuere deutsche Literatur streiten. In: Politycki 1998 a, S. 51-60 (Erstveröffentlichung: Frankfurter Rundschau, 06./07./08. 04. 1996).

Politycki, Matthias 2000: Der amerikanische Holzweg. In: Frankfurter Rundschau, 18. März, S. 2.

Polt-Heinzl, Evelyne 2006: Symbolfigur der neuen Erzählergeneration. Die Furche, 23. November, S. 8.

Polt-Heinzl, Evelyne 2013: Zwillingsgeschichten. Die Furche, 05. September, S.6f.

Proelß, Johannes 2005: Geschichte der Gartenlaube. In: Hamouda 2005, S. 65-144 (Erstveröffentlichung 1903).

Quintilianus, Markus Fabius 2011: Ausbildung des Redners. 12 Bücher. Lat./dt. Übers. u. hg. v. Helmut Rahn. Darmstadt: Wissenschaftliche Buchgesellschaft.

Regn, Gerhard 1992: Postmoderne und Poetik der Oberfläche. In: Klaus W. Hempfer (Hg.): Poststrukturalismus-Dekonstruktion-Postmoderne. Stuttgart: Steiner, S. 52-70. 
Reso, Martin 1965: Der geteilte Himmel und seine Kritiker. Halle: Mitteldeutscher Verlag.

Richardson, Brian 2007: Drama and narrative. In: Herman 2007 a, S. 142-155.

Richter, Hans Werner, und Mannzen, Walter (Hg.) 1964: Almanach der Gruppe 47 1947-1962. Reinbek bei Hamburg: Rowohlt.

Roth, Marco 2006: An escape from history. Daniel Kehlmann puts lightness into the Enlightenment. Time Literary Supplement, 15. Dezember, S.21.

Rowohlt 2014: [Der Rowohlt Verlag]. In: http://www.rowohlt.de/verlag (Zugang 31. 08. 2014).

Rubiner, Ludwig 1910: Dichter der Unwirklichkeit. Anmerkungen zu den Büchern des Max Brod. In: Der Sturm, 1:14, S. 107f.

Rüdenauer, Ulrich 2012: Munter durch die Kulissen geschubst. Süddeutsche Zeitung, 24./25. November, S. 17.

Ryan, Marie-Laure 2007: Toward a definition of narrative. In: Herman 2007 a, S. 22-35.

Schaffer, Tiz 2011: Das ist wirklich alles so passiert. Falter, 14. September, S. 52f.

Scheck, Denis 2005: Familienpackungen für Singles. Tagesspiegel, 20. November, S. 25.

Scheck, Denis 2006 a: Stunde der Schafe. Tagesspiegel, 28. Februar, S. 25.

Scheck, Denis 2006 b: Hass auf den ersten Blick. Tagesspiegel, 06. August, S. 26.

Scheck, Denis 2006 c: AOK oder Waffen-SS? Tagesspiegel, 03. September, S. 27.

Schiller, Friedrich 1943-2012: Schillers Werke. Nationalausgabe. Hg. v. Julius Petersen, Gerhard Fricke et. al. 43 Bde. Weimar: Böhlaus.

Schilling, Erik 2012: Der historische Roman seit der Postmoderne. Umberto Eco und die deutsche Literatur. Heidelberg: Winter.

Schirrmacher, Frank 1990: Abschied von der Literatur der Bundesrepublik. Frankfurter Allgemeine Zeitung, 02. Oktober, Literaturbeilage, S. 1.

Schirrmacher, Frank 1991: Dem Druck des härteren, strengeren Lebens standhalten. In: Deiritz/Kraus 1991, S. 127-136 (Erstveröffentlichung: Frankfurter Allgemeine Zeitung, 02. 06. 1990).

Schlegel, August Wilhelm, und Schlegel, Friedrich 1924: Athenäum. München: Meyer \& Tessen (Erstveröffentlichung: Berlin: Bey F. Vieweg dem Älteren 1798-1800). 
Schlegel, Friedrich 1958-: Kritische Friedrich-Schlegel-Ausgabe. 35 Bde. Hg. v. Ernst Behler. Paderborn: Schöningh.

Schmidt, Thomas E. 1997: Ein literarischer Pudel. Frankfurter Rundschau, 27. September, Beilage S. 4.

Schmitt, Elke 2004: Wörterwust statt Sprache. Der Spiegel, 16. Februar, S. 136-138.

Schneider, Susanne, et al. 2010: Wir müssen reden. Süddeutsche Zeitung Magazin, 22. Januar, S. 12-30.

Schulz, Georg-Michael 1997: Drei-Einheiten-Lehre. In: Weimar 1997-2003, Bd. 1, S. 408f.

Schwering, Markus 2012: Der Geist ist machtlos. Frankfurter Rundschau, 09. Oktober, S. 19.

Simon, Anne-Catherine 2008: Schreibst du a was Österreichisches. Die Presse, 29. Januar, S. 33+35.

Smith, Sidonie, und Watson, Julia (Hg.) 2010: Reading Autobiography. A Guide for Interpreting Life Narratives. 2. Aufl. Minneapolis: University of Minnesota Press (Erstveröffentlichung 2001).

Soboczynski, Adam 2012: Die Vermessung der Welt. Die Zeit, 23. August, S. 46.

Soyka, Otto 1910: Der Leser. In: Der Sturm, 1:33, S. 259 f.

Stein, Hannes 2007: Die Vermessung des Daniel Kehlmann. Die Welt, 09. November, S. 9.

Steiner, Bettina 2009: Meine Dämonen spuken zu Hause. Die Presse am Sonntag, 06. September, S. 48.

Steinert, Hajo 1994: Genie und Handwerk. In: Focus, 04. Februar, S. 88-90.

Strigl, Daniela 2013: Weltverdruss und Größenwahn. Falter, 28. August, S. 38.

Szyrocki, Marian (Hg.) 1977: Poetik des Barock. Stuttgart: Reclam.

Taberner, Stuart 2002: A New Modernism or 'Neue Lesbarkeit'? Hybridity in Georg Klein's Libidissi. German Life and Letters, 55:2, S. 137-148.

Taberner, Stuart (Hg.) 2005: German Literature of the 1990s and Beyond. Normalization and the Berlin Republic. New York: Camden House. 
Taberner, Stuart (Hg.) 2007: Contemporary German Fiction. Writing in the Berlin Republic. Cambridge: Cambridge University Press.

Thuswaldner, Anton 2009: Gegenaufklärung in der Literatur? Beilage zur Furche, 5. November S. 4-6.

Tieck, Ludwig (Hg.) 1848-1852: Kritische Schriften. 4 Bde. Leipzig: Brockhaus.

Troscianko, Emily 2014: Kafka’s Cognitive Realism. London: Routledge.

Türk, Joachim 1993: Netz aus Noten. Rhein-Zeitung, 22. Mai, S. 6.

Wackwitz, Stephan 1997: Totgesagte leben länger. Die Woche, 10. Oktober, S. 8.

Wagener, Hans 1988: Einleitung. In: Hunold 1988 a, S. 5-47.

Ward, Albert 1974: Book Production, Fiction and the German Reading Public 1740-1800. Oxford: Clarendon.

Weber, Max 1988: Der Sinn der 'Wertfreiheit' der soziologischen und ökonomischen Wissenschaften. In: Ders. (Hg.): Gesammelte Aufsätze zur Wissenschaftslehre. Tübingen: UTB, S. 489-540 (Erstveröffentlichung in: Logos. Internationale Zeitschrift für Philosophie der Kultur. Bd. 7. 1917/1918, S. 40-88).

Weimar, Klaus, et al. (Hg.) 1997-2003: Reallexikon der deutschen Literaturwissenschaft in drei Bänden. Berlin: De Gruyter.

Weimar, Klaus, und Solms, Friedhelm 1997: Geschmack. In: Weimar 1997-2003, Bd. 1, S. 714-717.

Wells, Benedict 2012: Und, wie geht die Geschichte weiter? Sag schon! In: Du, September, S. 34-37.

Weyrauch, Wolfgang (Hg.) 1949: Tausend Gramm. Sammlung neuer deutscher Geschichten. Hamburg: Rowohlt.

Wieland, Christoph Martin 1997f: Von der Freiheit der Literatur. Kritische Schriften und Publizistik. Hg. v. Wolfang Albrecht. 2 Bde. Frankfurt/M.: Insel.

Wiele, Jan 2013: Manche Wünsche sind zu groß, um in Erfüllung zu gehen. Frankfurter Allgemeine Zeitung, 23. August, S. 32.

Wiggershaus, Rolf 1987: Die Frankfurter Schule. Geschichte-Theoretische Entwicklung-Politische Bedeutung. München: Hanser.

Wittek, Bernd 1997: Der Literaturstreit im sich vereinigenden Deutschland. Eine Analyse des Streits um Christa Wolf und die deutsch-deutsche Gegenwartsliteratur in Zeitungen und Zeitschriften. Marburg: Tectum. 
Wittstock, Uwe 1995: Leselust. Wie unterhaltsam ist die neue deutsche Literatur? München: Luchterhand.

Wittstock, Uwe 2008: Die Realität und ihre Risse. Laudatio zur Verleihung des KleistPreises 2006 an Daniel Kehlmann. In: Nickel 2008, S. 113-126

(Erstveröffentlichung: Die Welt, 16. 12. 2006)

Wolf, Christa 2007: Was bleibt. Frankfurt/M.: Suhrkamp (Erstveröffentlichung 1990).

Woolf, Virginia 1984: The Common Reader. First Series. London: Hogarth Press (Erstveröffentlichung 1925).

Zweig, Stefan 1913: Das neue Pathos. Das neue Pathos, 1:1, S. 1-6. 